\title{
Methanophenazin: Strukturaufklärung und Totalsynthese eines neuartigen Cofaktors aus methanogenen Archaea
}

\author{
Dissertation \\ zur Erlangung des Doktorgrades \\ der Mathematisch-Naturwissenschaftlichen Fakultäten \\ der Georg-August-Universität zu Göttingen
}

vorgelegt von

Mario Tietze

aus Kyllburg

Göttingen 2000 
D7

Referent:

Priv.-Doz. Dr. U. Beifuss

Korreferent:

Prof. Dr. Dr. h.c. L. F. Tietze

Tag der mündlichen Prüfung:

02. 11.2000 
Die vorliegende Arbeit wurde in der Zeit von Oktober 1997 bis Mai 2000 am Institut für Organische Chemie der Georg-August-Universität in Göttingen angefertigt.

Besonders herzlich danke ich

Herrn Priv.-Doz. Dr. Uwe Beifuss für die interessante Themenstellung, die vielen Anregungen in zahlreichen Diskussionen und die Unterstützung und Förderung dieser Arbeit. 


\section{Inhaltsverzeichnis}

\section{Allgemeiner Teil}

$1 \quad$ Einleitung

2 Methanogenese 11

2.1 Methanogene Organismen 11

2.2 Der Energiestoffwechsel methanogener Archaea 13

2.3 Untersuchungen an Methanosarcina mazei Gö1 17

$3 \quad$ Zielsetzung und Planung der Arbeit 19

3.1 Strukturaufklärung von Methanophenazin (1) 20

3.2 Synthese von Methanophenazin (1) 20

3.3 Retrosynthese von Methanophenazin (1) 21

$4 \quad$ Strukturaufklärung von Methanophenazin (1) 24

$\begin{array}{lll}4.1 & \text { Fermentation } & 24\end{array}$

$\begin{array}{lll}4.2 & \text { Isolierung } & 24\end{array}$

4.3 Reinigung 25

4.4 Strukturaufklärung 31

$\begin{array}{lll}4.5 & \text { Zusammenfassung } & 42\end{array}$

$5 \quad$ Vorkommen und Biosynthese von Phenazinen 44

$5.1 \quad$ Vorkommen von Phenazinen 44

5.2 Biosynthese von Phenazinen 48

$6 \quad$ Synthese von 2-Hydroxyphenazin (2) 53

6.1 Wege zu Phenazinen 53

6.2 Synthese von 2-Hydroxyphenazin (2) nach Kehrmann und Cherpillod 56

6.3 Synthese von 2-Hydroxyphenazin (2) nach $\mathrm{Ott} \quad 58$

6.4 Diskussion der spektroskopischen Daten von 2-Hydroxyphenazin (2) 59 
$7 \quad$ Synthese des Phenazinethers rac-51 62

$7.1 \quad$ Optimierung der Versuchsbedingungen 62

7.2 Diskussion der spektroskopischen Daten von rac-51 64

$8 \quad$ Synthese der $C_{6}$-Bausteine rac-6 und $(R)-6 \quad 66$

$8.1 \quad$ Synthese von $r a c-6 \quad 66$

$\begin{array}{lll}8.2 & \text { Synthese von }(R)-6 & 67\end{array}$

8.2.1 Synthese der Lactone $(R)-\mathbf{1 2}$ und $(S)-\mathbf{1 2} \quad 69$

8.2.2 Öffnung des Lactons $(R)-\mathbf{1 2}$ zum Amid $(R)-\mathbf{5 6} \quad 71$

8.2.3 Diskussion der spektroskopischen Daten von $(R)-56 \quad 71$

8.2.4 Reduktion des Piperidids $(R)-56$ und Synthese von $(R)-6 \quad 73$

8.2.5 Diskussion der spektroskopischen Daten von $(R)-6 \quad 75$

8.3 Bestimmung der optischen Reinheit des $\mathrm{C}_{6}$-Bausteins $(R)-6 \quad 76$

$\begin{array}{lll}8.4 & \text { Zusammenfassung } & 79\end{array}$

$9 \quad$ Synthese des diastereomerenreinen $\mathrm{C}_{19}$-Bausteins $7 \quad \mathbf{8 0}$

9.1 Synthese des terminalen Alkins $9 \quad 80$

9.2 Diskussion der spektroskopischen Daten von 9

$9.3 \quad$ Synthese des Vinyliodids $7 \quad 84$

9.4 Diskussion der spektroskopischen Daten von 786

$\begin{array}{lll}9.5 & \text { Zusammenfassung } & 88\end{array}$

$10 \quad$ Synthese von Methanophenazin (1) 89

10.1 Palladium-katalysierte Kreuzkupplung von $(R)-6$ mit 7

10.2 Desilylieren der Seitenkette von $(S)$-76 91

10.3 Bestimmung der Enantiomerenreinheit von $(S)-78 \quad 92$

10.4 Diskussion der spektroskopischen Daten von $(S)-78 \quad 95$

10.5 Darstellung von $(S)$-Methanophenazin $[(S)-\mathbf{1}] \quad 98$

10.6 Darstellung von racemischem Methanophenazin rac-1 99

10.7 Diskussion der spektroskopischen Daten von

(S)-Methanophenazin [(S)-1] 99

$\begin{array}{lll}10.8 \quad \text { Zusammenfassung } & 103\end{array}$ 
11 Versuche zur Ermittlung der absoluten Konfiguration $\begin{array}{ll}\text { von Methanophenazin (1) } & 104\end{array}$

11.1 Untersuchungen mit chromatographischen Methoden 104

$\begin{array}{ll}11.2 & \text { Untersuchungen mit chiroptischen Methoden }\end{array}$

11.3 Versuche zur Aufklärung der absoluten Konfiguration durch $\begin{array}{ll}\text { chemischen Abbau } & 106\end{array}$

$\begin{array}{lll}11.4 & \text { Zusammenfassung } & 107\end{array}$

12 Die biologische Funktion von Methanophenazin (1) 108

12.1 Untersuchungen zum membrangebundenen Elektronentransfer in Methanosarcina mazei Gö1 110

$\begin{array}{lll}12.2 & \text { Zusammenfassung } & 113\end{array}$

13 Zusammenfassung und Ausblick $\quad 114$

\section{Experimenteller Teil}

$1 \quad$ Allgemeine Verfahren $\quad 125$

$1.1 \quad$ Allgemeine Arbeitstechniken 125

$\begin{array}{lll}1.2 & & 125\end{array}$

$\begin{array}{lll}1.3 & \text { Chromatographische Methoden } & 126\end{array}$

$\begin{array}{lll}1.4 & \text { Software } & 127\end{array}$

$2 \quad$ Synthese von 2-Hydroxyphenazin (2) 128

$2.1 \quad 1,2,4$-Triacetoxybenzol (44) 128

2.2 1,2,4-Trihydroxybenzol (45) 129

$2.3 \quad$ 2-Hydroxyphenazin (2) nach Kehrmann und Cherpillod 130

$2.4 \quad$ 2-Hydroxyphenazin (2) nach $O t t \quad 131$ 
$3 \quad$ Synthese des Phenazinethers rac-51 133

$3.1 \quad(3 R S)-( \pm)-8-M e t h y l s u l f o n y l-2,6-$ dimethyl-non-2-en (rac-49) 133

$3.2(3 R S)-( \pm)-8$-Toluolsulfonyl-2,6-dimethylnon-2-en (rac-48) 134

3.3 (3RS)-( \pm )-8-Brom-2,6-dimethyl-2-hepten (rac-50) 135

$3.4 \quad\left(3^{\prime} R S\right)-( \pm)-2-(3,7-D i m e t h y l-o c t-6-e n y l o x y)-p h e n a z i n(r a c-51) \quad 136$

$4 \quad$ Synthese des $\mathrm{C}_{6}$-Bausteins rac-6 138

4.1 (3RS)-( \pm )-5-tert.-Butylsilyloxy-3-methyl-pentan-1-ol (rac-52) 138

$4.2 \quad(3 R S)-( \pm)-5$-Iodo-3-methyl-pentoxy-isopropyl-

dimethyl-silan (rac-6)

4.3 (3RS)-( \pm )-5-Bromo-3-methyl-pentoxy-isopropyl-

dimethyl-silan (rac-53)

$5 \quad$ Synthese der $C_{6}$-Bausteine $(R)-6$ und $(S)-12 \quad 142$

$5.1(4 R)-(+)-4-M e t h y l-t e t r a h y d r o-2-p y r a n-2-o n[(R)-12] \quad 142$

$5.2 \quad(3 R)-(+)-5-H y d r o x y-3-m e t h y l-1-p i p e r i d i n-$

1-yl-pentan-1-on $[(R)-56]$

5.3 (3R)-(-)-5-tert.-Butyldimethylsilyloxy-3-methyl-

1-yl-pentan-1-on $[(R)-57] \quad 144$

5.4 (3S)-(+)-5-tert.-Butylsilyloxy-3-methyl-pentan-1-ol [(R)-52] 145

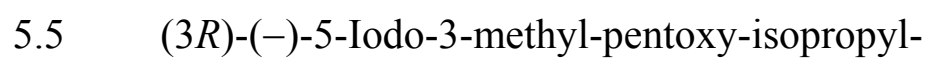

dimethyl-silan $[(R)-6]$

5.6 Synthese des Lactons (4S)-(-)-4-Methyl-tetrahydro-

pyran-2-on $[(S)-12]$

$6 \quad$ Synthese des diastereomerenreinen Vinyliodids 7

$6.1 \quad(5 E, 9 E)-6,10,14-$ Trimethyl-pentadeca-5,9,13-trien-1-in (9)

$6.2(1 E, 5 E, 9 E)$-1-Iodo-2,6,10,14-tetramethyl-pentadeca-

$1,5,9,13$-tetraen $(7)$ 
$7 \quad$ Synthese von racemischen Methanophenazin (rac-1)

153

$7.1 \quad(6 E, 10 E, 14 E)-(3 R S)-( \pm)-t e r t .-B u t y l o x y s i l y l-3,7,11,15,19$ -

pentamethyl-eicosa-6,10,14,18-tetraen (rac-76)

$7.2(6 E, 10 E, 14 E)-(3 R S)-( \pm)-3,7,11,15,19-P e n t a m e t h y l-e i c o s a-$

6,10,14,18-tetraen-1-ol (rac-78)

$7.3 \quad\left(E 6^{\prime}, E 10^{\prime}, E 14^{\prime}\right)-\left(3^{\prime} R S\right)-( \pm)-2(3,7,11,15,19-P e n t a m e t h y l-e i c o s a-$

6,10,14,18-tetraenyloxy)-phenazin ( $r a c-1)$

8 Synthese von $(S)$-Methanophenazin [(S)-1]

159

$8.1 \quad(6 E, 10 E, 14 E)-(3 S)-(-)-3,7,11,15,19-P e n t a m e t h y l-e i c o s a-$

6,10,14,18-tetraen-1-ol [(S)-78]

$8.2 \quad\left(E 6^{\prime}, E 10^{\prime}, E 14^{\prime}\right)-\left(3^{\prime} S\right)-(-)-2(3,7,11,15,19-P e n t a m e t h y l-e i c o s a-$

6,10,14,18-tetraenyloxy)-phenazin $[(S)-1]$

9 Synthese der Mosher-Ester rac-63, $(S, S)-63$, rac 79 und $(S, S)-79$

9.1 (2S,3'RS)-3,3,3-Trifluoro-2-methoxy-2-phenyl-propansäure-

5'-tert.-butyldimethylsilyl-pentylester (rac-63)

9.2 (2S,3'S)-3,3,3-Trifluoro-2-methoxy-2-phenyl-propansäure-

5'-tert.-butyldimethylsilyl-pentylester $[(S)-63]$

$9.3\left(2 S, 3^{\prime} R S, 6^{\prime} E, 10^{\prime} E, 14^{\prime} E, 18^{\prime} E\right)$-3,3,3-Trifluoro-2-methoxy-2-phenyl-propansäure-3',7',11',15',19'-pentamethyl-eicosa-tetraenyl-ester ( $r a c-79)$

9.4 (2S,3' $\left.S, 6^{\prime} E, 10^{\prime} E, 14^{\prime} E, 18^{\prime} E\right)$-3,3,3-Trifluoro-2-methoxy-2-phenyl-propansäure-3',7',11',15',19'-pentamethyl-eicosa-tetraenyl-ester [(S,S)-79]

10 Charakterisierung von Methanophenazin (1)

167

\section{Anhang}

1 Abkürzungsverzeichnis

169

2 Literaturverzeichnis und Anmerkungen

170 
I Allgemeiner Teil 


\section{$1 \quad$ Einleitung}

Die physikalische Grundeinheit aller Organismen ist die Zelle. Sie ist die kleinste lebensfähige Einheit und setzt sich im wesentlichen aus den Bestandteilen Desoxyribonucleinsäure (DNA), Ribonucleinsäure (RNA), Proteinen, Lipiden und Phospholipiden zusammen. Jede vegetative Zelle hat eine definierte Struktur und Größe und stellt ein mikroskopisch und makroskopisch hochgeordnetes System dar, dessen Struktur die Grundlage des Lebens ist. Die Zelle ist ein offenes System, das nach Art eines Fließgleichgewichts kontinuierlich Material aufnimmt, transformiert und ausscheidet. Durch den hohen Organisationsgrad befinden sich lebende Organismen nicht im Gleichgewicht mit ihrer Umgebung, die dem dritten Hauptsatz der Thermodynamik folgend, auf Maximierung der Entropie des Universums ausgerichtet ist. Um einen Ordnungszustand aufzubauen und zu erhalten, muß Energie aufgewendet werden, weshalb vegetative Zellen sowohl während des Wachstums als auch in Ruhe auf dauernde Energiezufuhr angewiesen sind. ${ }^{[1,2]}$

Die zur Erhaltung des Lebens sowie zur Neusynthese von Zellbestandteilen und Reduplikation der Zelle notwendige Energie gewinnt der Organismus im Stoffwechsel. Hierbei werden Nährstoffe aus der Umgebung aufgenommen und mit Hilfe von Enzymen umgesetzt. Man unterscheidet den Katabolismus, bei dem durch den Abbau von organischen Substanzen niedermolekulare Verbindungen gebildet und biochemisch nutzbare Energie freigesetzt wird, vom Anabolismus, bei dem aus niedermolekularen Verbindungen durch biologische Synthese monomere und polymere Zellbestandteile aufgebaut werden.

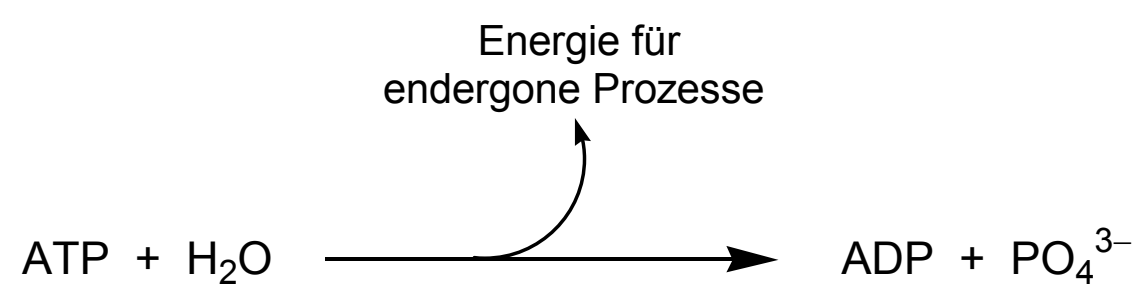

Abb. 1: Umwandlung von ATP in ADP und Phosphat

Das Coenzym Adenosin-5'-triphosphat (ATP) stellt die wichtigste Speicherform biologisch nutzbarer Energie dar und ist der universelle Überträger zwischen energiefreisetzenden und energieaufwendigen Reaktionen. Beinahe alle energieaufwendigen Prozesse in lebenden Zellen sind mit der Umwandlung von ATP zu Adenosin-5'-diphosphat (ADP) gekoppelt (Abbildung 1). ${ }^{[3]}$ 
Die Hydrolyse von ATP führt unter Abspaltung eines Phosphatrestes $\mathrm{PO}_{4}{ }^{3-} \mathrm{zu}$ ADP und ist stark exergon. Die dabei freigesetzte chemische Energie dient dazu, durch energetische Kopplung endergone Vorgänge wie Biosynthesen, Bewegungs- und Transportprozesse zu ermöglichen.

Im Laufe der Evolution haben sich zwei grundlegende Mechanismen der ATP-Synthese herausgebildet, die in ähnlicher Weise in allen Zellen stattfinden. Unter der Elektronentransportphosporylierung versteht man einen Prozess, bei dem Elektronen von einem Donor mit negativem Redoxpotential auf einen Akzeptor mit stärker positivem Redoxpotential übertragen werden. Diese Redoxreaktionen verlaufen immer an Membranen, wobei die freiwerdende Energie zum Aufbau eines transmembranen elektrochemischen Gradienten genutzt wird. Wie in einem Kondensator findet an der Membran eine Trennung der Ladungen statt, die der Speicherung von Energie dient. Wichtig für die zelluläre ATP-Synthese sind Protonengradienten. Der Energiegehalt $\Delta \mathrm{G}$ eines solchen Gradienten ist die Summe aus der pH-Differenz $\Delta \mathrm{pH}$ und des elektrischen Membranpotentials $\Delta \Psi$ zwischen der inneren und äußeren Seite der Membran. Das Membranpotential $\Delta \Psi$ ist direkt abhängig von der Differenz der Redoxpotentiale von Elektronendonor und Elektronenakzeptor. Aus den beiden Größen $\Delta \mathrm{pH}$ und $\Delta \Psi$ ergibt sich die protonenmotorische Kraft $\Delta \mathrm{P}$ als Maß für die chemische Arbeit $\Delta \mathrm{G}$, die der Gradient zu leisten vermag. ${ }^{[4]}$

$\mathrm{H}^{+}$- transportierende, membrangebundene ATP-Synthasen nutzen den aufgebauten Protonengradienten und dessen Energie zur ATP-Synthese. Elektronentransportphosphorylierung findet in der Atmungskette und im Photosyntheseapparat statt.

Der zweite Mechanismus der ATP-Bildung wird als Substraphosphorylierung bezeichnet. Er beruht auf der Kopplung der ATP-Synthese an die Hydrolyse energiereicher Stoffwechselmetabolite, die beim Abbau von organischen Substraten in kleiner Zahl gebildet werden. Solche Verbindungen wie beispielsweise Phosphoenolpyruvat, Acetylphosphat und 1,3-Bisphosphoglycerat enthalten energiereiche Phosphatbindungen (Abbildung 2).<smiles></smiles>

1,3-Bisphosphoglycerat<smiles>[R6]OC(=C)C(=O)O</smiles>

Phosphoenolpyruvat<smiles></smiles>

Acetylphosphat

Abb. 2: Strukturen von 1,3 Bisphosphoglycerat, Phosphoenolpyruvat und Acetylphosphat 
Im Verlauf des Stoffwechsels ermöglichen Enzyme den Transfer der energiereichen Phosphatgruppen vom Substrat auf ADP. Beispielweise katalysiert das Enzym PyruvatKinase die Übertragung einer Phosphatgruppe von Phosphoenolpyruvat auf ADP. Bei dieser Reaktion entstehen Pyruvat und ATP (Abbildung 3).

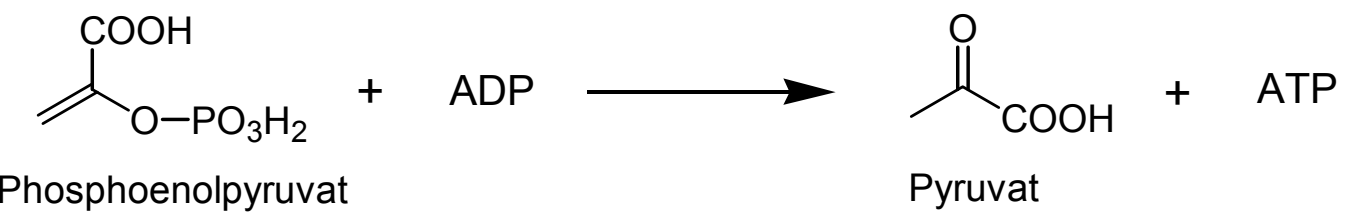

Abb. 3: Umwandlung von Phosphoenolpyruvat in Pyruvat und ATP

Es hat sich als nützlich erwiesen, die Organismen nach der Art der Energiequellen einzuteilen, die sie nutzen, um nach den genannten Mechanismen ATP zu regenerieren.

Demnach unterscheidet man die phototrophen von den chemotrophen Organismen. Diejenigen Organismen, welche als Energiequelle das Licht nutzen, werden als phototroph bezeichnet. Chemotrophe Lebewesen hingegen gewinnen ihre Energie zur ATP-Regeneration aus Redox-Reaktionen an den als Nährstoffe dienenden Substraten (Abbildung 4). ${ }^{[3]}$

Weitere Kriterien zur Einteilung der Lebewesen berücksichtigen neben der Energiequelle die Kohlenstoffquelle und den Wasserstoff-Donor des Organismus. Mit Blick auf den Ursprung des Zellkohlenstoffs unterscheidet man zwischen heterotrophen und autotrophen Lebewesen. Die Erstgenannten nutzen organische Verbindungen, letztgenannte nutzen Kohlendioxid als Kohlenstoffquelle zum Zellaufbau. Alle Organismen, die organische Verbindungen als Wasserstoff-Donor benutzen, werden als organotroph bezeichnnet. Der Begriff lithotroph kennzeichnet die Fähigkeit, den Wasserstoff aus anorganischen Donoren wie elementarem Wasserstoff $\left(\mathrm{H}_{2}\right)$, Ammoniak $\left(\mathrm{NH}_{3}\right)$, Schwefelwasserstoff $\left(\mathrm{H}_{2} \mathrm{~S}\right)$ u. a. verwerten zu können. Phototrophe Pflanzen und Cyanobakterien, die den anorganischen Elektronendonor Wasser $\left(\mathrm{H}_{2} \mathrm{O}\right)$ zur Reduktion von Kohlendioxid verwenden, werden demnach als photolithoautotroph bezeichnet. Organismen, die im Licht mit organischen Substraten wachsen, werden photorganoheterotroph genannt. Dementsprechend bezeichnet man chemotrophe Bakterien, die organisches Material benutzen, als chemorganoheterotroph. $\mathrm{Zu}$ dieser Gruppe gehören anaerobe und aerobe Bakterien. Die Anaeroben nutzen entweder Nitrat $\left(\mathrm{NO}_{3}{ }^{-}\right)$, Sulfat $\left(\mathrm{SO}_{4}{ }^{2-}\right)$ oder organische Verbindungen als Elektronenakzeptor. Chemolithoautotrophe Bakterien bedienen sich anorganischer Elektronendonoren wie Wasserstoff $\left(\mathrm{H}_{2}\right)$, Ammoniak $\left(\mathrm{NH}_{3}\right)$, Schwefelwasserstoff $\left(\mathrm{H}_{2} \mathrm{~S}\right)$ u. a. und oxidieren diese mit Luftsauerstoff zu Wasser, Sulfat 
$\left(\mathrm{SO}_{4}{ }^{2-}\right)$ und Nitrit $\left(\mathrm{NO}_{2}{ }^{-}\right)$. Diese exergonische Reaktion wird mit der Bildung von ATP aus ADP und Phosphat gekoppelt. ${ }^{[1]}$

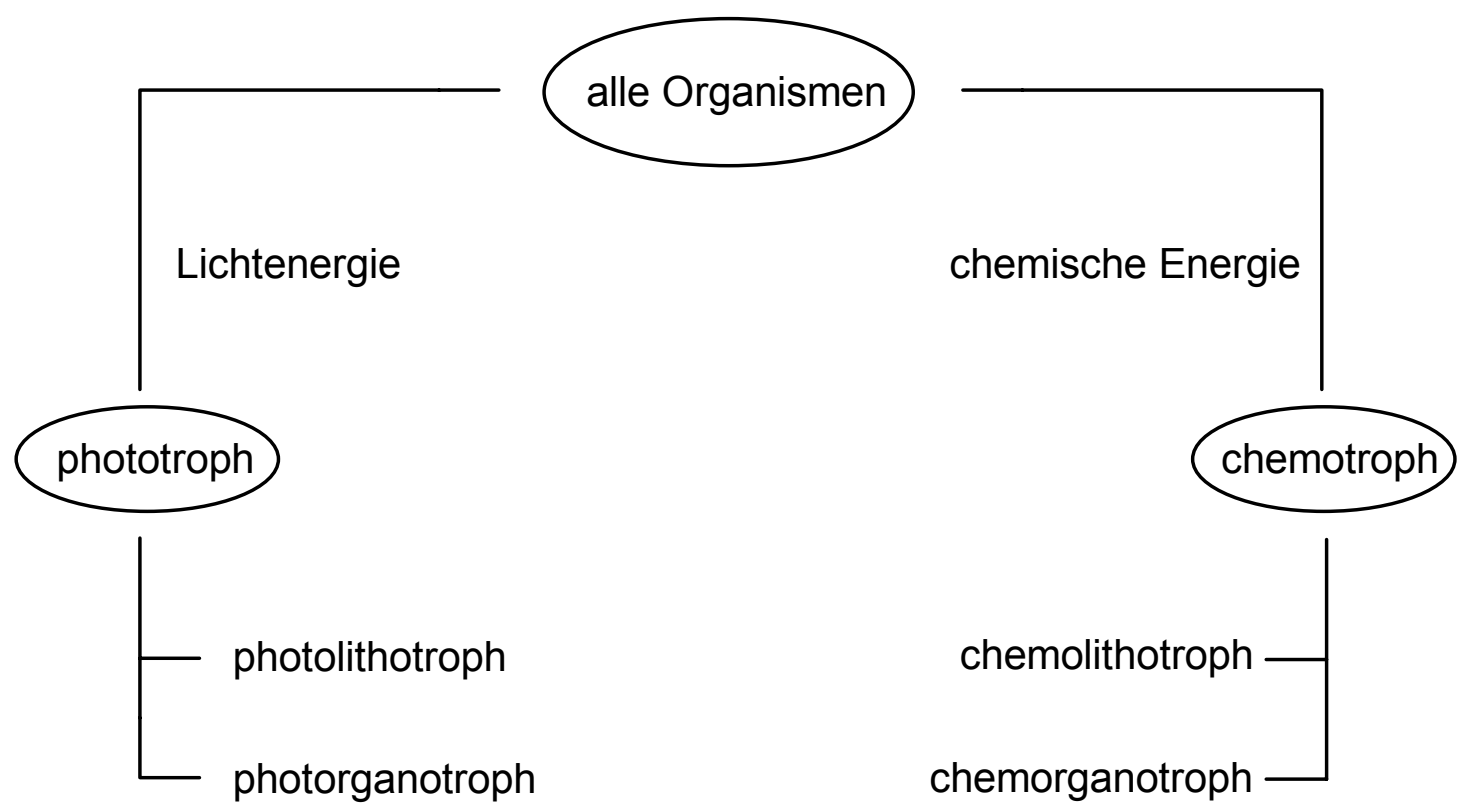

Abb. 4: Schema zur Einteilung der Lebewesen nach der Energie- und Kohlenstoffquelle die sie zum Leben nutzen ${ }^{[5]}$

Die Methanogenen unterscheiden sich deutlich von allen anderen chemolithoautotrophen Bakterien, weil sie im Gegensatz zu diesen strikt anaerobe Organismen sind. Sie gewinnen ATP durch Reduktion von Kohlendioxid zu Methan mit molekularem Wasserstoff. Da sie keine organischen Verbindungen, sondern nur anorganische Substrate zur Energiegewinnung einsetzen und ihre einzige Kohlenstoffquelle aus Kohlendioxid besteht, sind sie ihrer Lebensweise entsprechend in die Gruppe chemolithoautotropher Archaea einzuordnen.

Kohlenhydrate sind die mengenmäßig vorherrschenden Produkte pflanzlicher Photosynthese und zugleich die allgemeinen Nahrungsstoffe für die überwiegende Zahl von Mikroorganismen. Die meisten chemorganotrophen Bakterien und alle höheren Organismen nutzen sie als Kohlenstoff- und Energiequelle. Unter aeroben Bedingungen wird ein Teil des Substrats unter Energiegewinn zu Kohlendioxid und Wasser transformiert. Sauerstoff bildet dabei den terminalen Elektronenakzeptor. Die freigesetzte Energie dient der Synthese von monomeren und polymeren Zellbestandteilen. 
Der am weitesten verbreitete Abbauweg verläuft über Fructose-1,6-bisphosphat als Zwischenverbindung. Wichtige alternative Abbauwege sind der Pentosephosphat-Weg, auf dem Glucose zu Ribulose-5-phosphat umgesetzt wird und der 2-Keto-3-desoxy-6phosphogluconat-Weg, der die gleichnamige Zwischenverbindung zu Glycerinaldehyd-3phosphat und Pyruvat spaltet (Abbildung 5).

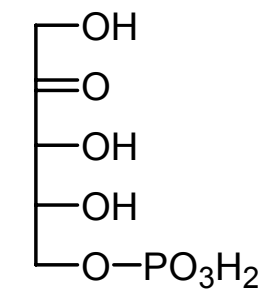

Ribulose-5-phosphat

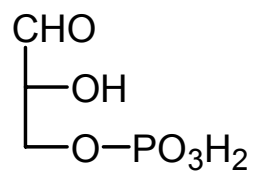

Glycerinaldehyd-3-phosphat

Abb. 5: Strukturen von Ribulose-5-phosphat und Glycerinaldehyd-3-phosphat

Beim Transport in die Zelle wird Glucose in Glucose-6-phosphat, ihre stoffwechselaktive Form, übergeführt. Sie ist der Ausgangspunkt der drei oben genannten Abbauwege (Abbildung 6). Durch den auch als Glycolyse bekannten Fructose-1,6-bisphosphat-Weg wird Glucose-6-phosphat zu zwei Molekülen Pyruvat abgebaut. Neben vier Reduktionsäquivalenten (Protonen und Elektronen), die auf $\mathrm{NAD}^{+}$übertragen NADH ergeben, werden im Sinne einer Substratphosphorylierung noch zwei Moleküle ATP gebildet. Die Verwertung der $\mathrm{C}_{3}$-Bausteine Pyruvat erfolgt durch einen Multienzymkomplex, der als Pyruvat-Dehydrogenase bezeichnet wird. Unter oxidativer Decarboxylierung entsteht als wichtiges Endprodukt Acetyl-CoA. Es fallen weitere vier Reduktionsäquivalente an, die auf $\mathrm{NAD}^{+}$übertragen werden. Der Tricarbonsäure-Cyclus (TCC) bewirkt unter Abspaltung des Wasserstoffs die vollständige Oxidation von Acetat $\mathrm{zu}$ Kohlendioxid. Von den 16 Reduktionsäquivalenten werden 12 auf $\mathrm{NAD}^{+}$und zwei auf das Coenzym FAD übertragen. Es entstehen zwei Moleküle ATP durch Substratphosphorylierung. Der Tricarbonsäure-Cyclus hat nicht nur die Funktion der terminalen Oxidation der Nahrungsstoffe, sondern stellt auch zahlreiche Vorstufen für die Biosynthese zur Verfügung. Auffüllungsreaktionen, sogenannte anaplerotische Sequenzen, gleichen einen Verbrauch von Intermediaten des TricarbonsäureCyclus aus. ${ }^{[1]}$ Die Bilanz der Oxidation von Glucose lautet: ${ }^{[6]}$

$$
\mathrm{C}_{6} \mathrm{H}_{12} \mathrm{O}_{6}+6 \mathrm{H}_{2} \mathrm{O}+4 \mathrm{ADP}+4 \mathrm{PO}_{4}{ }^{3-} \longrightarrow 6 \mathrm{CO}_{2}+24[\mathrm{H}]+4 \mathrm{ATP}
$$



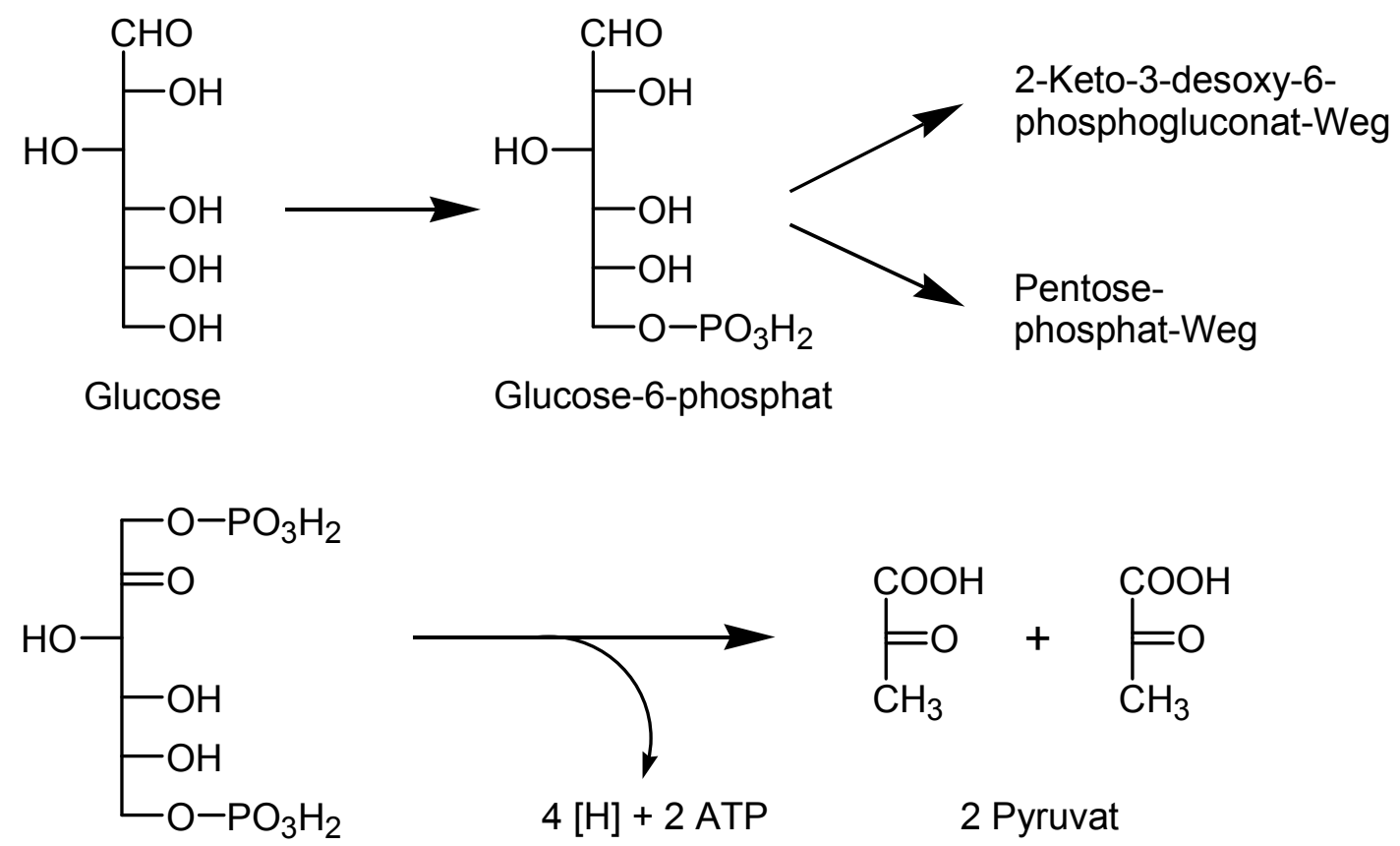

Fructose-1,6-bisphosphat

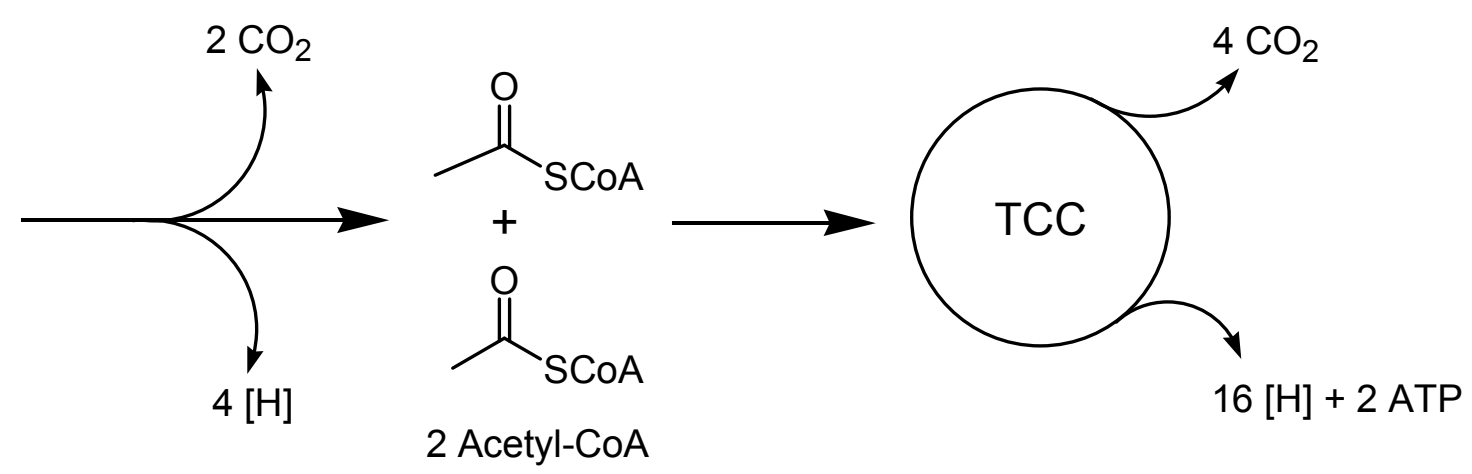

Abb. 6: Schema zum Abbau von Glucose auf dem Fructose-1,6-bisphosphat-Weg ${ }^{[1]}$

Die von der Oxidation der organischen Substrate stammenden Reduktionsäquivalente werden von Coenzymen, meist $\mathrm{NAD}^{+}$als NADH, aufgenommen und in die Elektronentransportkette der aeroben Organismen eingeschleust. Bei Prokaryonten trägt die Cytoplasmamembran die Atmungskette, bei Eukaryonten die innere Mitochondrienmembran. Die Atmungskette umfaßt drei Proteinkomplexe (Komplexe I, III und IV), die in die Membran eingebaut sind. ${ }^{[2]}$ Alle Komplexe der Elektronentransportkette sind aus zahlreichen Polypeptid-Untereinheiten aufgebaut und enthalten proteingebunden eine Reihe verschiedener Redox-Coenzyme wie Flavine (FMN oder FAD), Eisen-Schwefelzentren (Fe/S) und Häm-Gruppen. Bei der Oxidation von NADH durch Komplex I gelangen die Elektronen über FMN und Fe/S-Zentren zum Ubichinon. Es wird dadurch zum Hydrochinon reduziert (Abbildung 7). 
<smiles>CCC(C)=CCC1=C(C)C(=O)C(OC)=C(OC)C1=O</smiles>

Ubichinon

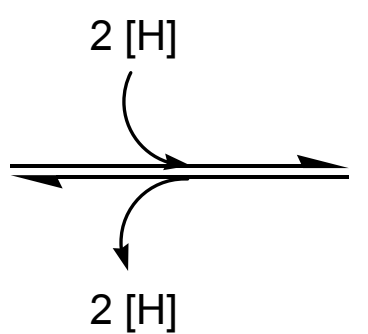

$2[\mathrm{H}]$<smiles>CCCC=CCc1c(C)c(O)c(OC)c(OC)c1O</smiles>

Ubihydrochinon

Abb. 7: oxidierte und reduzierte Form von Ubichinon, $n=6-10$

Durch seine unpolare isoprenoide Seitenkette kann sich das Hydrochinon $\left(\mathrm{QH}_{2}\right)$ in der Membran bewegen und gelangt so zu Komplex III, wo es zu Ubichinon (Q) oxidiert wird. Seiner Natur gemäß wird Ubichinon als membrangebundener Elektronen-Carrier bezeichnet.

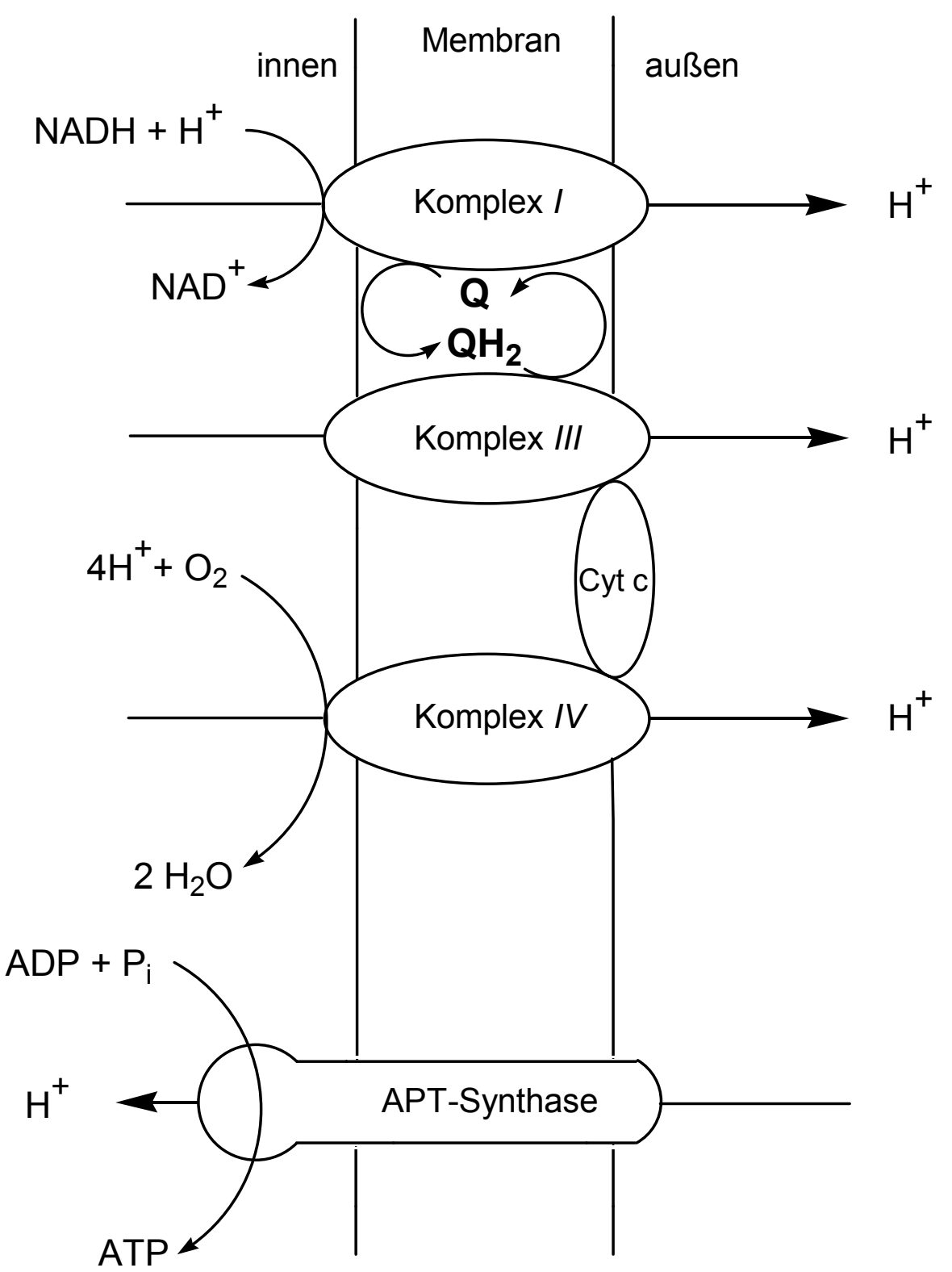

Abb. 8: Vereinfachtes Modell der Atmungskette in Eukaryonten und Prokaryonten ${ }^{[2]}$ 
Über das Häm-Protein Cytochrom-c gelangen die Elektronen von Komplex III zu Komplex $I V$, der sie auf Sauerstoff als terminalen Elektronenakzeptor überträgt. In der Atmungskette vollzieht sich die „,biologische Knallgasreaktion“ (Abbildung 8).

Die Elektronen werden in der Weise durch die Membran geleitet, daß es zur ProtonenTranslokation und zum Aufbau eines transmembranen elektrochemischen Gradienten kommt, mit einem positiven Potential auf der Außenseite und einem negativen Potential auf der Innenseite der Membran. Katalysiert durch die ATP-Synthase wird die protonenmotorische Kraft von der Zelle zur ATP-Regeneration eingesetzt. Die Energiebilanz der GlucoseVeratmung lautet: ${ }^{[6]}$

$$
6 \mathrm{O}_{2}+24[\mathrm{H}]+34 \mathrm{ADP}+34 \mathrm{PO}_{4}{ }^{3-} \longrightarrow 12 \mathrm{H}_{2} \mathrm{O}+34 \mathrm{ATP}
$$

Zusammen mit den vier Äquivalenten ATP durch Substratphosphorylierung aus der Glycolyse und dem Tricarbonsäure-Cyclus ergeben sich 38 ATP für den Gesamtprozeß aus Glucose-Oxidation und Veratmung. Es wird deutlich, daß die Elektronentransport-Kette zusammen mit der APT-Synthase den wirksamsten Apparat zur ATP-Regeneration darstellt.

Überall dort in der Natur, wo organische Verbindungen vorliegen, es aber an Sauerstoff fehlt, wie beispielsweise in den Sedimenten von Flüssen und Seen oder im Verdauungssystem von Wiederkäuern, setzen sich anaerobe Mikroorganismen durch. Das Fehlen von Sauerstoff als terminalem Elektronenakzeptor hat entscheidende Auswirkungen auf den Elektronenfluß und die Energiekonservierung. Bei den Gärungen wird kein externer Elektronenakzeptor genutzt. Stattdessen dient das organische Substrat als Wasserstoff-Donor und Wasserstoff-Akzeptor zugleich. Außerdem wird es als Baustoff für die Biosynthese genutzt. Gärprozesse bewirken eine Disproportionierung des Substrats. ${ }^{[6]}$

Durch oxidativen Abbau organischer Substrate, vorwiegend über den Fructose-1,6bisphosphat-Weg, entsteht unter ATP-Bildung zunächst Pyruvat als wichtigste Zwischenverbindung. Als Akzeptoren des in Form von NADH vorliegenden Wasserstoffs dienen Zwischenprodukte des Substratabbaus. Bei der Regeneration von $\mathrm{NAD}^{+}$werden diese reduziert und die Reduktionsprodukte aus den Zellen ins Medium ausgeschieden. So werden beispielweise bei der homofermentativen Milchsäure-Gärung aus einem Mol Glucose zwei Mol Milchsäure und zwei Mol ATP gebildet. 


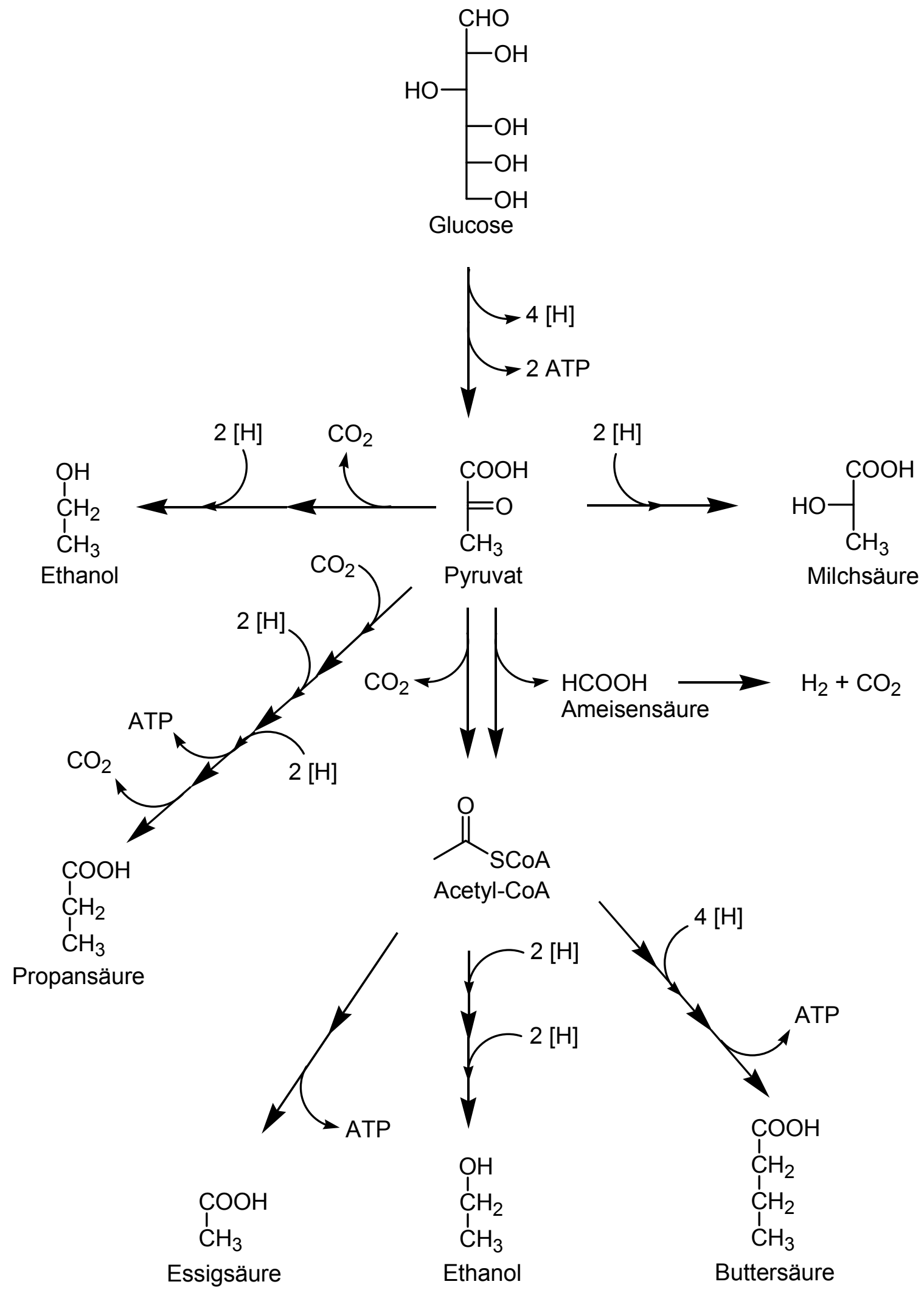

Abb. 9: Übersicht über Verlauf und Produkte der wichtigsten Gärungen ${ }^{[6]}$ 
Die Energiekonservierung erfolgt bei Gärungen ausschließlich durch Substratphosphorylierung. Im Vergleich mit der aeroben Atmung bleiben ATP-Ausbeute und Wachstumsertrag bei den Gärern gering. Die Zellen setzen große Mengen an Substrat um, bevor sie sich teilen können. Bei der Vergärung von Kohlenhydraten und einigen anderen Substraten treten u. a. Ethanol, Milchsäure, Ameisensäure, Essigsäure, Propansäure, Buttersäure, Wasserstoff und Kohlendioxid als Produkte auf (Abbildung 9).

Die Gärer haben am Stoffwechselkreislauf in der Natur einen wesentlichen Anteil, denn sie leiten die anaerobe Nahrungskette ein. Die vergorenen organischen Biomonomere und Biopolymere liefern fast immer Wasserstoff, der im Mittelpunkt der anaeroben Nahrungskette steht und den methanbildenden und sulfatreduzierenden Bakterien als Substrat dient, das sie zur Generierung von Methan und Schwefelwasserstoff benötigen.

Unter anaeroben Bedingungen kommt es neben der Gärung häufig zur Entwicklung von Bakterien, die ihre Stoffwechselenergie durch ,anaerobe Atmung“ gewinnen. Als Kohlenstoffquellen und Wasserstoff-Donatoren verwerten sie die Stoffwechselprodukte der gärenden Bakterien. Die anaeroben Atmer stehen am Ende der anaeroben Atmungskette und sind für den Prozeß der Mineralisation von entscheidender Bedeutung. Als WasserstoffAkzeptoren vermögen sie Nitrat $\left(\mathrm{NO}_{3}{ }^{-}\right)$, Sulfat $\left(\mathrm{SO}_{4}{ }^{2-}\right)$, Carbonat $\left(\mathrm{CO}_{3}{ }^{2-}\right)$, Kohlendioxid $\left(\mathrm{CO}_{2}\right)$ und andere Verbindungen zu verwerten (Tabelle 1). ${ }^{[1]}$

Tabelle 1: Anaerobe Atmungsprozesse

$$
\begin{array}{lll}
10[\mathrm{H}]+2 \mathrm{NO}_{3}{ }^{-}+2 \mathrm{H}^{+} \rightarrow \mathrm{N}_{2}+6 \mathrm{H}_{2} \mathrm{O} & \text { Denitrifikation } & \text { Nitrat-Atmung } \\
8[\mathrm{H}]+\mathrm{SO}_{4}{ }^{2-}+2 \mathrm{H}^{+} \rightarrow \mathrm{H}_{2} \mathrm{~S}+4 \mathrm{H}_{2} \mathrm{O} & \text { Sulfat-Reduktion } & \text { Sulfat-Atmung } \\
2[\mathrm{H}]+\mathrm{S} \rightarrow \mathrm{H}_{2} \mathrm{~S} & \text { Schwefel-Reduktion } & \text { Schwefel-Atmung } \\
8[\mathrm{H}]+2 \mathrm{CO}_{2} \rightarrow \mathrm{CH}_{3} \mathrm{COOH}+2 \mathrm{H}_{2} \mathrm{O} & \mathrm{CO}_{2} \text {-Reduktion zu Acetat } & \text { Carbonat-Atmung } \\
8[\mathrm{H}]+\mathrm{CO}_{2} \rightarrow \mathrm{CH}_{4}+2 \mathrm{H}_{2} \mathrm{O} & \mathrm{CO}_{2} \text {-Reduktion zu Methan } & \text { Carbonat-Atmung } \\
{[\mathrm{H}]+\mathrm{Fe}^{3+} \rightarrow \mathrm{Fe}^{2+}+\mathrm{H}^{+}} & \text {Eisen-Reduktion } & \text { Eisen-Atmung }
\end{array}
$$

Die wichtigste Carbonat-Atmung ist die Methanogenese. Hierbei wird Kohlendioxid vollständig zu Methan reduziert. Die Methanogenese wird ausschließlich von Archaea geleistet und soll im nachfolgenden Kapitel ausführlich dargestellt werden. 


\section{Methanogenese}

\subsection{Methanogene Organismen}

Die Bildung von Methan ist einer der bedeutendsten biologischen Prozesse im Zuge des Abbaus von organischem Material unter anaeroben Bedingungen. ${ }^{[7]}$ Die Hydrolyse polymerer organischer Materie zu Monomeren und deren fermentative Umsetzung durch gärende Organismen liefert einfache Verbindungen wie Wasserstoff, Kohlendioxid, Ameisensäure, Essigsäure, Methanol und Methylamine als Substrat für methanogene Archaea. Sie stehen am Ende der anaeroben Atmungskette und transformieren die genannten Substrate zu Methan und Kohlendioxid, die als gasförmige Produkte dem Kohlenstoffkreislauf wieder zugeführt werden. Der Gehalt an Methan in der Atmosphäre beträgt etwa 1.8 ppm. Nach Schätzungen werden rund $70 \%$ davon durch biologische Methanogenese-Prozesse gebildet. ${ }^{[8]}$

Methanogene Organismen zählen zu den Archaea, einem Organismenreich, das sich aus den extrem thermophilen und thermoaciden Archaea (Crenarchaeota) sowie den extrem halophilen und methanogenen Archaea (Euryarchaeota) zusammensetzt. Das Reich der Archaea bildet eine eigenständige Evolutionslinie im phylogenetischen Stammbaum (Abbildung 10). Diese besondere Stellung wird aus 16S-rRNA-Analysen deutlich, anhand derer der Unterschied zu den Eukaryonten und Bakteria sichtbar wird. Im Gegensatz zu den Bacteria besteht die archaeelle Zellwand nicht aus einer Mureinschicht, sondern aus Pseudomurein, Heteropolysacchariden und Proteinen. ${ }^{[9]}$ Die Cytoplasmamembran von Archaea enthält anstelle von Fettsäureglycerinestern eine Reihe von $\mathrm{C}_{20^{0}}$-Diphytanyl-und $\mathrm{C}_{40^{-}}$ Biphytanyl-Glycerinethern. ${ }^{[10]}$ Die Komponenten, die an der Proteinbiosynthese von Archaebakterien beteiligt sind, zeigen evolutionäre Nähe zu den Eukarya. ${ }^{[11]}$ Betrachtungen des Zellstoffwechsels und die Art und Weise der Energiegewinnung lassen Übereinstimmungen mit den Bacteria erkennen.

Für die strikt anaeroben methanogenen Archaea bilden Sumpf- und Tundrengebiete, Sedimente von Seen und Flüssen sowie Wiederkäuerpansen und die Faultürme von Kläranlagen den natürlichen Lebensraum. 


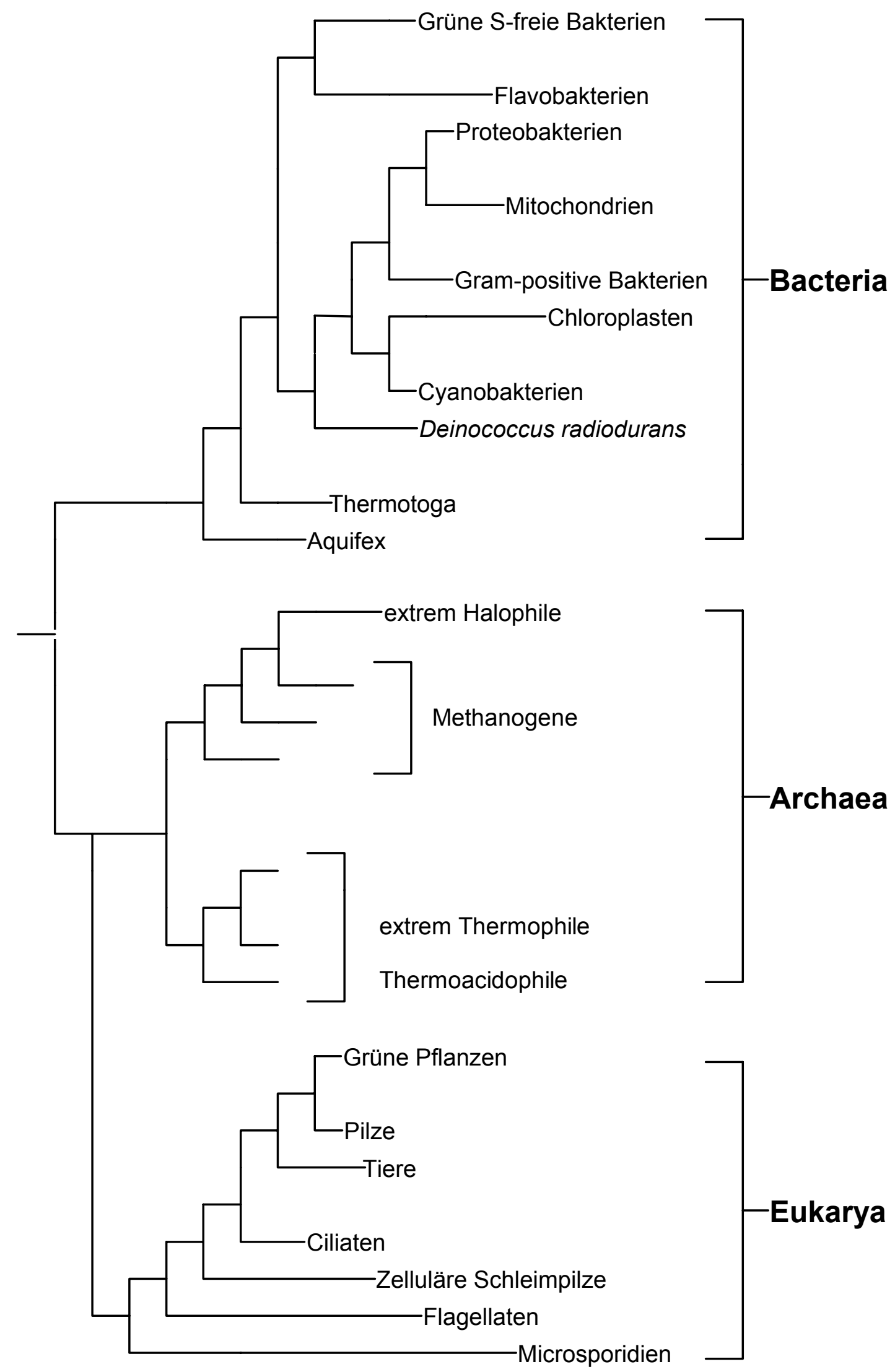

Abb. 10: Der phylogenetische Stammbaum aller Lebewesen nach H. Cypionka ${ }^{[12]}$ 


\subsection{Der Energiestoffwechsel methanogener Archaea}

Die Gruppe der Methanogenen umfasst zahlreiche, morphologisch uneinheitliche Organismen, die alle ausschließlich Methanogenese zur Energiegewinnung betreiben. Hydrogenotrophe Methanogene sind in der Lage Wasserstoff zu aktivieren und Kohlendioxid zu fixieren. Im Zuge der Energiegewinnung wird Wasserstoff oxidiert und Kohlendioxid zu Methan reduziert. Ihre Lebensweise wird als chemolithoautotroph bezeichnet, da sie Kohlendioxid als einzige Kohlenstoffquelle zum Aufbau von Zellsubstanz nutzen. Andere Vertreter dieser Gruppe, die methylotrophen Methanogenen, verwerten einfache $\mathrm{C}_{1}$ Verbindungen wie Ameisensäure, Methanol und Methylamin zur Energiegewinnung. Das Substratspektrum acetogenotropher Methanogener beschränkt sich auf Essigsäure, die zu Methan und Wasserstoff transformiert wird. ${ }^{[13]}$

Methanogene Archaea der Gattung Methanosarcina sind in der Lage, Wasserstoff und Kohlendioxid gemäß nachfolgender Gleichung zu Methan umzusetzen.

$$
\mathrm{CO}_{2}+4 \mathrm{H}_{2} \rightarrow \mathrm{CH}_{4}+2 \mathrm{H}_{2} \mathrm{O}
$$

Wie Studien an den Cytoplasmamembranen von Methanosarcina mazei und Methanosarcina barkeri ergaben, werden die hierbei ablaufenden Redox-Reaktionen von membrangebundenen Proteinkomplexen katalysiert. ${ }^{[14]}$ Neben diesen spezifischen Enzymsystemen sind in die Kohlendioxid-Reduktion auch einige ungewöhnliche Cofaktoren involviert (Abbildung 13). ${ }^{[15]}$

Die Methanogenese wird durch die Aktivität des Formylmethanofuran-DehydrogenaseSystems eingeleitet (Abbildung 11). Dabei wird Kohlendioxid zunächst von Methanofuran (MF) fixiert und dann reduktiv als Formyl-Methanofuran (Formyl-MF) gebunden. Eine anschließende Formylgruppenübertragung auf Tetrahydromethanopterin $\left(\mathrm{H}_{4} \mathrm{MPT}\right)$ ergibt 5Formyl-H${ }_{4} \mathrm{MPT}$. Die Formylgruppe bleibt im weiteren Verlauf an $\mathrm{H}_{4} \mathrm{MPT}$ gebunden und wird schrittweise bis zum Methylrest reduziert. Als Reduktionsmittel fungiert das Coenzym $\mathrm{F}_{420} \mathrm{H}_{2}$, das durch die $\mathrm{F}_{420}$-abhängige Dehydrogenase bereitgestellt wird. Die Methylgruppe von Methyl- $\mathrm{H}_{4}$ MPT wird auf HS-CoM übertragen, bevor die Methyl-Co-M-Reduktase die HS-CoB-abhängige Reduktion des zuvor gebildeten Methyl-S-Co-M zum Methan katalysiert. Bei dieser reduktiven Demethylierung von Methyl-S-CoM fällt das gemischte Disulfid CoMS-S-CoB als Reaktionsprodukt an. ${ }^{[16]}$ Es dient der membrangebundenen $\mathrm{H}_{2}$ :CoB-S-S-CoMOxidoreduktase als terminaler Elektronenakzeptor. Die beiden an der Reaktion beteiligten Cofaktoren werden dabei durch die Tätigkeit der Heterodisulfidreduktase regeneriert. 


\section{Cytoplasmamembran}

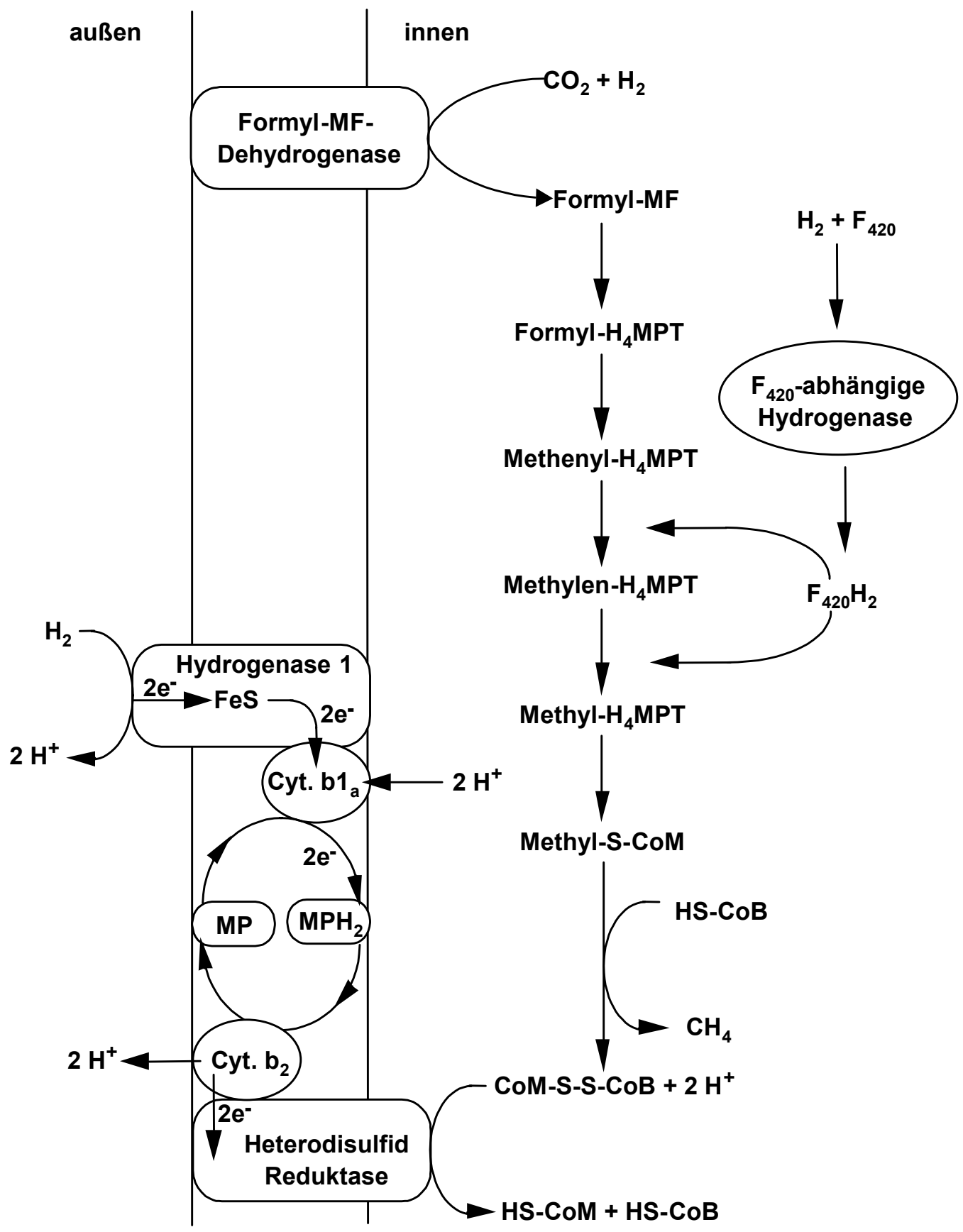

Abb. 11: Modell des Energiestoffwechsels und des Elektronentransfers bei hydrogenotrophen Methanogenen nach Deppenmeier und Gottschalk ${ }^{[17,22]}$ 
Die $\mathrm{H}_{2}$ :CoB-S-S-CoM-Oxidoreduktase-Reaktion ist mit dem Aufbau eines transmembranen Protonengradienten verbunden. ${ }^{[18]}$ Er wird durch die Aktivität einer ATP-Synthase zur ATPSynthese genutzt.

Bei der Methanogenese aus Methanol (Abbildung 12) handelt es sich um eine Disproportionierung von Methanol, gemäß nachfolgender Reaktionsgleichung.

$$
4 \mathrm{CH}_{3} \mathrm{OH} \rightarrow 3 \mathrm{CH}_{4}+\mathrm{CO}_{2}+2 \mathrm{H}_{2} \mathrm{O}
$$

Der erste Schritt bei dieser Umsetzung ist eine durch Methyltransferasen (MT1, MT2) katalysierte Übertragung der Methylgruppe von Methanol auf das Coenzym HS-CoM wobei sich Methyl-CoM bildet. ${ }^{[19]}$ An dieser Stelle spaltet sich der Stoffwechsel in einen reduktiven und einen oxidativen Zweig auf. Im reduktiven Abschnitt wird Methyl-S-CoM analog dem Vorgang beim hydrogenotrophen Stoffwechsel, mit Hilfe der Methyl-CoM-Reduktase, in Gegenwart von HS-CoB zu Methan und CoB-S-S-CoM transformiert. Die Reduktion des Heterodisulfids erfolgt wiederum durch die Heterodisulfid-Reduktase. ${ }^{[20]}$

Der zweite Abschnitt beschreibt die Oxidation der Methylgruppe zum Kohlendioxid und beginnt mit dem Methyltransfer auf Tetrahydromethanopterin ( $\left.\mathrm{H}_{4} \mathrm{MPT}\right)$ durch die membranintegrale Methyl-H 4 MPT:CoM-Methyltransferase. Nach dem Methyltransfer erfolgt die sukzessive Oxidation von Methyl- $\mathrm{H}_{4} \mathrm{MPT}$ in entgegengesetzter Richtung zum hydrogenotrophen Stoffwechsel. Abschließend ermöglicht die FormylmethanofuranDehydrogenase die Oxidation von Formyl-Methanofuran zu Kohlendioxid. Die bei der Oxidation des Methylrestes anfallenden Reduktionsäquivalente werden auf das Coenzym $\mathrm{F}_{420}$ übertragen. Die Oxidation von $\mathrm{F}_{420} \mathrm{H}_{2}$ ist mit der Reduktion des Heterodisulfids CoM-S-SCoM gekoppelt. ${ }^{[21]}$ Diese exergone Reaktion ist mit dem Aufbau eines elektrochemischen Protonengradienten verbunden, der zur ATP-Synthese genutzt wird. 


\section{Cytoplasmamembran}

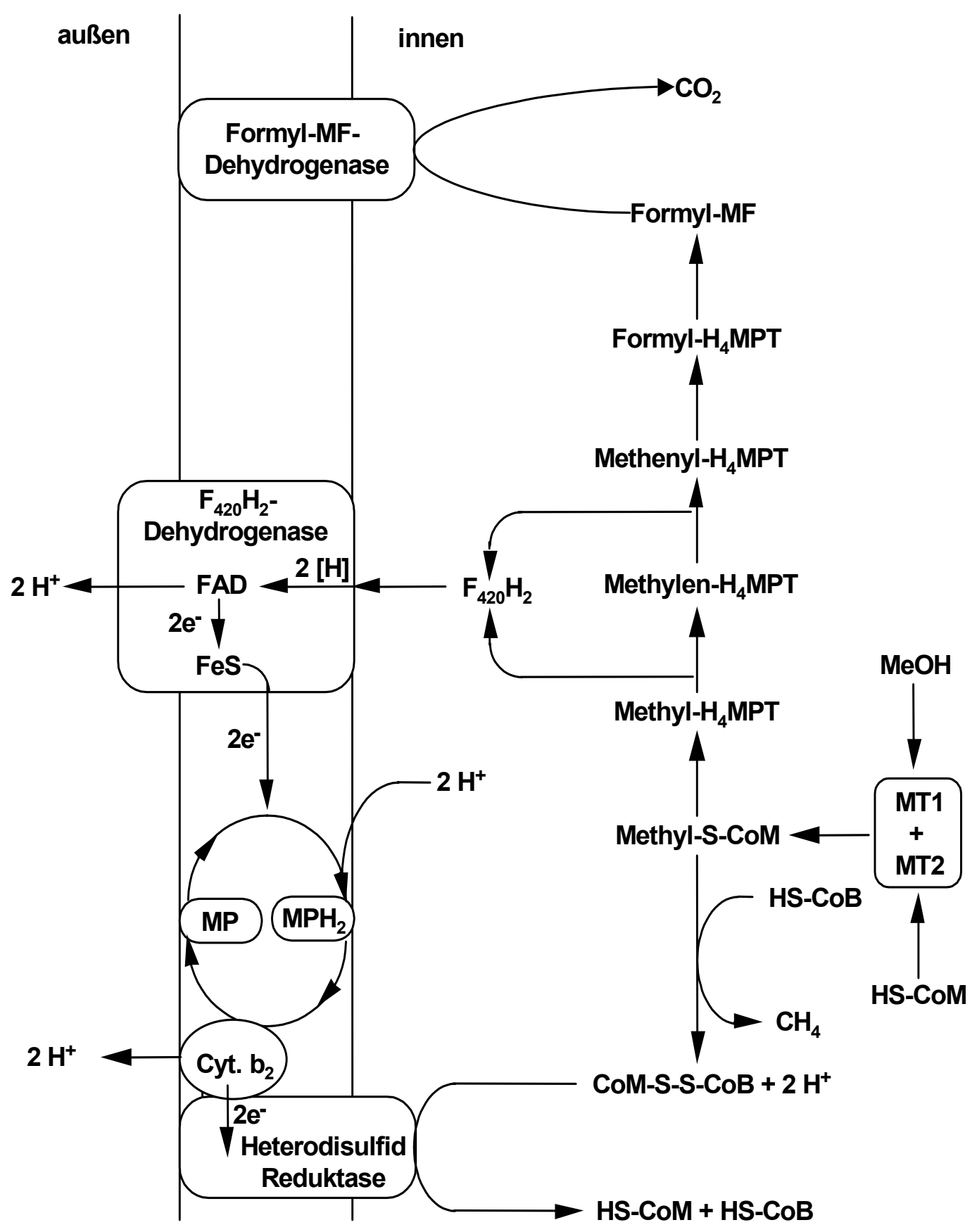

Abb. 12: Modell des Energiestoffwechsels und des Elektronentransfers bei methylotrophen Methanogenen nach Deppenmeier und Gottschalk ${ }^{[17,22]}$ 


\subsection{Untersuchungen an Methanosarcina mazei Gö1}

Der Stamm Methanosarcina mazei Gö1 verfügt über eine reichhaltige Enzymausstattung und vermag Wasserstoff und Kohlendioxid, sowie Methanol, Methylamine und Essigsäure als Substrat zu nutzen. Die Untersuchungen an diesem Organismus konzentrierten sich in den letzten Jahren auf die beiden protonentranslozierenden Elektronentransportsysteme. ${ }^{[23]}$ Die $\mathrm{F}_{420} \mathrm{H}_{2}:$ Heterodisulfid-Oxidoreduktase steht hierbei an zentraler Stelle bei den hydrogenotrophen Methanogenen. Sie ist aus zwei Komponenten, der $\mathrm{F}_{420}$-Dehydrogenase und der Heterodisulfid-Reduktase zusammengesetzt.

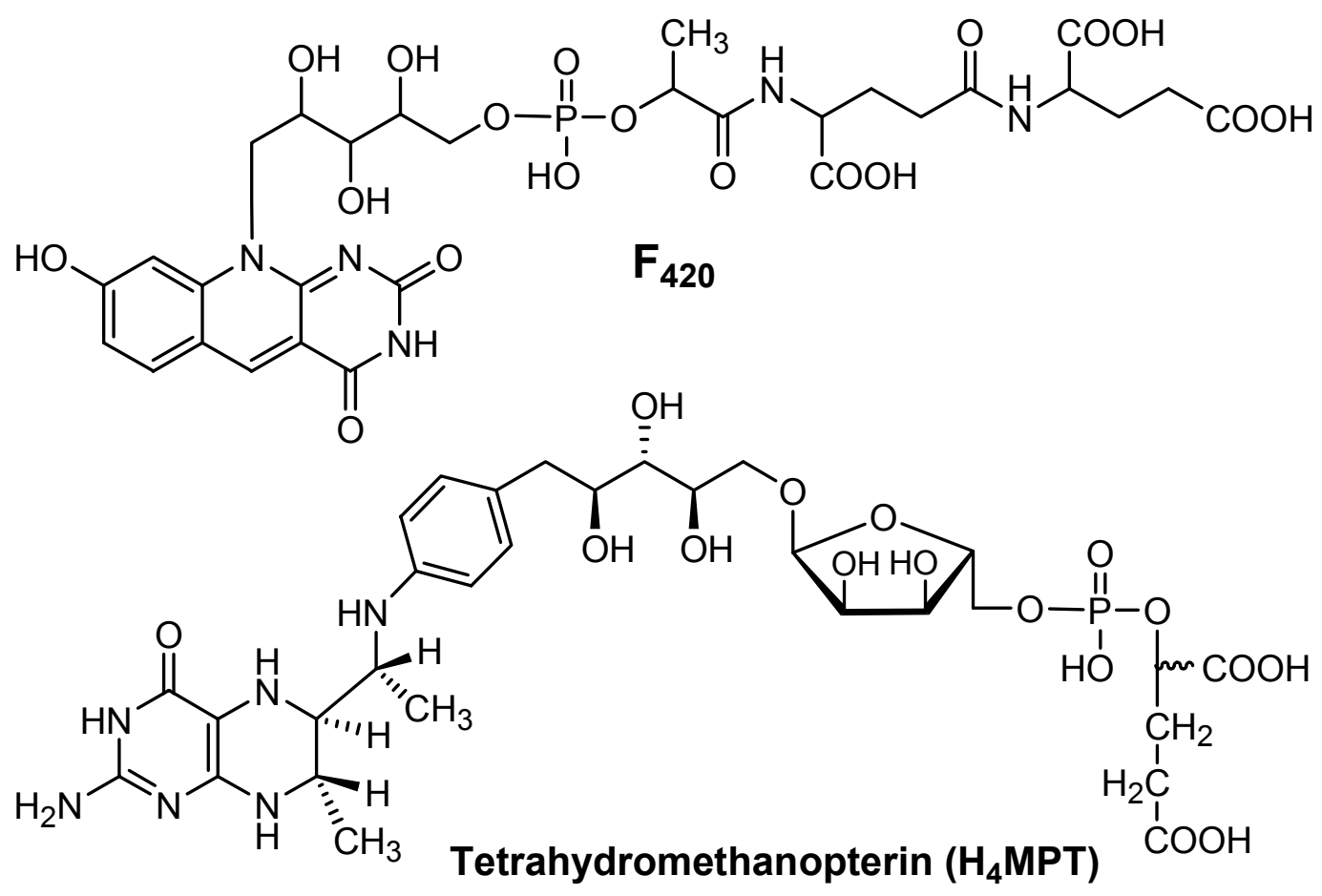<smiles>CCCCOC(C)(C)C(C)(C)C</smiles><smiles>CC(CC(C)(C)S)C(=O)N[C@H](C(=O)O)[C@@H](C)OP(=O)(O)O</smiles>

HS-CoB<smiles>SCCS[SeH3-]</smiles>

HS-CoM

Abb. 13: Strukturformeln der an der Methanogenese beteiligten Coenzyme ${ }^{[16,120]}$ 
Kürzlich gelang die Isolierung und Reinigung der $\mathrm{F}_{420}$-Dehydrogenase aus Ms. Mazei. ${ }^{[24,25]}$ Das Protein besitzt eine Masse von 400 kDa, ist aus 13 Untereinheiten aufgebaut und enthält neben Eisen-Schwefel-Clustern auch FAD. Bei der zweiten Komponente dieses Systems handelt es sich um die Heterodisulfid-Reduktase, deren Charakterisierung zeigt, daß sie aus zwei Untereinheiten aufgebaut ist. ${ }^{[26]}$ Bei der kleinen Untereinheit handelt es sich um ein bTyp-Cytochrom.

Das $\mathrm{H}_{2}$ :Heterodisulfid-Oxidoreduktase-System aus $M s$. mazei übernimmt eine Schlüsselfunktion im Elektronentransport und ist aus zwei membrangebundenen Proteinen konstruiert. Eine Hydrogenase, die Eisen-Schwefel-Cluster enthält und eine Cytochrom-b Untereinheit trägt, repräsentiert eine Komponente des Systems. ${ }^{[27]}$ Der zweite Teil des Elektronentransportsystems besteht aus der bereits oben angeführten HeterodisulfidReduktase.

Der Cofaktor $\mathrm{F}_{420}$ ist der zentrale Elektronenüberträger im Stoffwechsel der Methanogenen und in seiner Funktion vergleichbar mit dem Coenzym $\mathrm{NAD}^{+}$bzw. $\mathrm{NADP}^{+}$in Eubakterien und Eukaryonten. $\mathrm{F}_{420}$ dient unter anderem als Cofaktor der $\mathrm{F}_{420}$ abhängigen Hydrogenase, der Methylen- $\mathrm{H}_{4}$ MPT-Dehydrogenase sowie der Methylen- $\mathrm{H}_{4} \mathrm{MPT}$-Reduktase und tritt in reduzierter Form als $\mathrm{F}_{420} \mathrm{H}_{2}$ direkt mit der $\mathrm{F}_{420} \mathrm{H}_{2}$-Dehydrogenase in Kontakt.

Im Gegensatz dazu war die Natur des membrangebundenen Elektronenüberträgers, der den Transport der Elektronen zwischen der $\mathrm{F}_{420} \mathrm{H}_{2}$-Dehydrogenase bzw. Hydrogenase und der Heterodisulfid-Reduktase vermittelt, lange Zeit unbekannt. Während in Eubakterien diese Aufgabe von Chinonen wahrgenommen wird, findet man bei methanogenen Organismen nur Tocopherolchinone, die nicht am Elektronentransport beteiligt sind.

Die vorliegende Arbeit berichtet über die Entdeckung eines Phenazin-Derivates als neuartigem, membranintegralem Cofaktor aus Methanosarcina mazei Gö1. Auf die Reinigung, Strukturaufklärung, Synthese und biologische Funktion des als Methanophenazin (1) bezeichneten Naturstoffs wird in den nachfolgenden Kapiteln ausführlich eingegangen. 


\section{$3 \quad$ Zielsetzung und Planung der Arbeit}

Wie im vorangehenden Abschnitt ausführlich erläutert, setzt sich das $\mathrm{F}_{420} \mathrm{H}_{2}$-abhängige Heterodisulfid-Reduktasesystem von Methanosarcina mazei aus der $\mathrm{F}_{420}$-abhängigen Hydrogenase und einer Heterodisulfid-Reduktase zusammen. Das System ermöglicht die Energiekonservierung beim Leben auf einfachen Substraten wie Methanol oder Methylamin. Des weiteren besitzt der Organismus das $\mathrm{H}_{2}$ :Heterodisulfid-Oxidoreduktase-System, wodurch Wasserstoff und Kohlendioxid zur Energiekonservierung verfügbar gemacht werden. Es ist aus einer Hydrogenase und der Heterodisulfid-Reduktase aufgebaut. In beiden Systemen reduziert die Heterodisulfid-Reduktase das gemischte Disulfid CoB-S-S-CoM, das bei der Methanerzeugung im Organismus anfällt. Diese Reduktion ist mit einer Protonentranslokation und dem Aufbau eines transmembranen Protonengradienten verknüpft, der von einer ATPSynthase zur ATP-Regeneration genutzt wird (Abbildung 14).

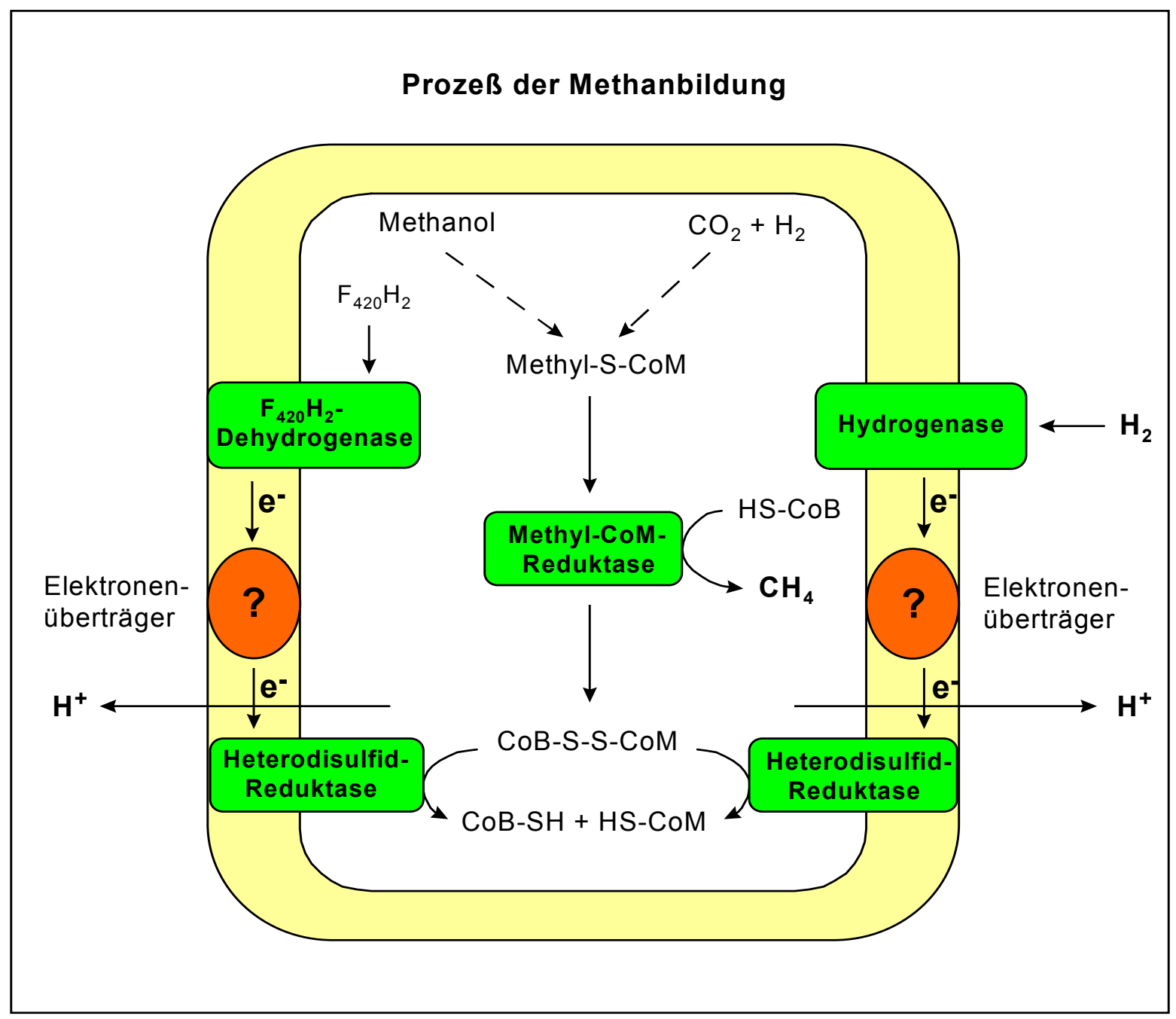

Abb. 14: Vereinfachtes Schema der Methanogenese in Methanosarcina mazei Gö1 


\subsection{Strukturaufklärung von Methanophenazin (1)}

Auf der Suche nach einem unbekannten, membranintegralen Elektronenüberträger, der für die Übertragung der Elektronen von der $\mathrm{F}_{420} \mathrm{H}_{2}$-Dehydrogenase bzw. der Hydrogenase auf die Heterodisulfid-Reduktase verantwortlich ist, extrahierte Abkendie Cytoplasmamembranen von Ms. mazei Gö1 mit Isooctan. ${ }^{[24]}$

Eine Analyse des Rohextrakts, die Separation der Bestandteile aus der Etherlipid-Matrix und die Reinigung der abgetrennten Bestandteile sowie deren Strukturaufklärung definierten ein erstes Ziel der vorliegenden Arbeit.

Mit der Isolierung geringer Mengen des als Methanophenazin (1) bezeichneten, redoxaktiven Naturstoffs 1 und der Aufklärung seiner Konstitution durch massenspektrometrische und NMR-spektroskopische Methoden, wurde der erste Abschnitt der Arbeit erfolgreich abgeschlossen.

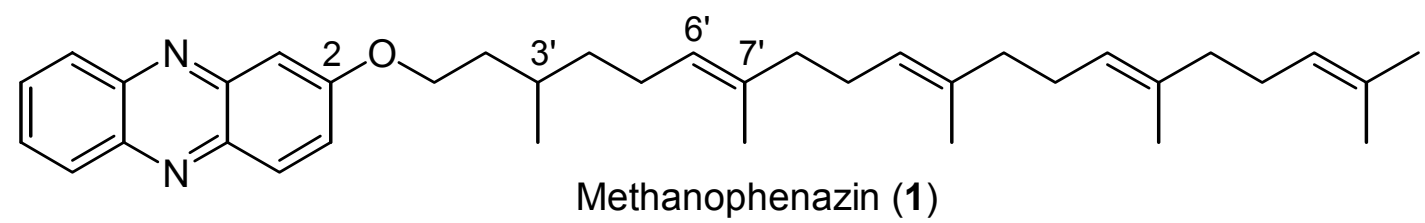

Es handelt sich bei Methanophenazin (1) nicht nur um das erste Phenazin-Derivat das jemals aus Archaea isoliert wurde, sondern auch um das erste Phenazin-Derivat überhaupt, dem eine Funktion am Elektronentransport in einer Atmungskette nachgewiesen wurde. ${ }^{[28,29]}$

\subsection{Synthese von Methanophenazin (1)}

Zur Bestimmung der absoluten Konfiguration des natürlichen Methanophenazins (1) sowie zur Untersuchung seiner Rolle im membrangebundenen Elektronentransport von Ms. mazei waren größere Mengen des Naturstoffs erforderlich. Die durch Extraktion aus Cytoplasmamembranen erhältlichen Mengen an Methanophenazin (1) sind zu gering und der Aufwand ihrer Isolierung und Reinigung zu groß, als daß sich der Bedarf an $\mathbf{1}$ aus dieser natürlichen Quelle decken ließe. Eine Synthese des Naturstoffs war also unabdingbar.

Ein Problem bei der Strukturaufklärung der Seitenkette betrifft das Auftreten eines stereogenen Zentrums in Position C-3'. Die Ermittlung seiner absoluten Konfiguration erfordert die Synthese des Naturstoffs 1 in racemischer und enantiomerenreiner Form. Nur ein Vergleich der synthetischen Verbindung bekannter Konfiguration mit dem natürlich 
vorkommenden Material ermöglicht eine eindeutige Zuordnung und die Aufklärung des Stereozentrums.

Zur Lösung der angegebenen Probleme war es also nötig, eine Synthesestrategie zu entwickeln, die sowohl die Synthese von racemischen Methanophenazin ( $r a c-1)$ als auch die von enantiomerenreinem $(R)$ - und $(S)$-1 gestattet.

\subsection{Retrosynthese von Methanophenazin (1)}

Eine retrosynthetische Analyse von $r a c-1$ führt $\mathrm{zu}$ 2-Hydroxyphenazin (2) und der terpenoiden Einheit $\mathrm{rac}-\mathbf{3}$, welche durch Etherbildung miteinander verknüpft werden können (Abbildung 15). Der Baustein rac-3 läßt sich in das Alkyliodid rac-6 und das (E)-Vinyliodid 7 zerlegen. Eine $\operatorname{Pd}(0)$ katalysierte $\mathrm{sp}^{3}-\mathrm{sp}^{2}$ Kupplungsreaktion soll die Verknüpfung von rac-6 mit 7 gewährleisten und die (Z)-Geometrie der C-6', C-7'-Doppelbindung sicherstellen. Das (E)-Vinyliodid (7) kann mit der von Negishi und seinen Mitarbeitern erarbeiteten Methode zur Carboaluminierung aus dem Alkin 9 zugänglich gemacht werden, das sich auf $(E, E)$ Farnesylaceton (10) zurückführen läßt. ${ }^{[30]}$ Die Synthese von 2-Hydroxyphenazin (2) sollte nach der Methode von Kehrmann und Cherpillod durch Umsetzung von 2-Hydroxychinon (4) mit $o$-Phenylendiamin (5) hergestellt werden. ${ }^{[31]}$ 
<smiles>CC(C)=CCC/C(C)=C/CC/C(C)=C/CC/C(C)=C/CC[C@@H](C)CCOc1ccc2nc3ccccc3nc2c1</smiles>

rac-1<smiles>Oc1ccc2nc3ccccc3nc2c1</smiles><smiles>CCCC(C)CC</smiles><smiles>[CH-]1[CH-]CCC1</smiles><smiles>COCCC(C)CCC=C(C)CCC=C(C)CCC=C(C)CCC=C(C)C</smiles>

2

rac-3<smiles>CCC(C)C</smiles><smiles>O=C1C=CC(=O)C(O)=C1</smiles>

4

5

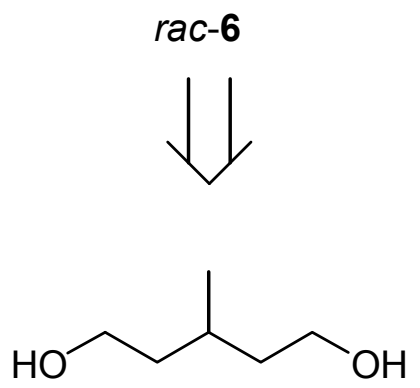

8<smiles>C#CCC/C=C(/C)CC/C=C(/C)CCC=C(C)C</smiles>

9<smiles>CC(C)=CCCC(C)=CCCC(C)=CCCC(C)C</smiles>

10

Abb. 15: Retrosyntheseschema von Methanophenazin (rac-1)

Fragment rac-6 sollte sich in wenigen Stufen aus 3-Methylpentan-1,5-diol (8) durch selektive Funktionalisierung der beiden Hydroxylgruppen aufbauen lassen. Der Vorteil eines solchen symmetrischen $\mathrm{C}_{6}$-Bausteins besteht in der Möglichkeit, durch Differenzierung seiner 
enantiotopen Gruppen die beiden Enantiomere $(R)-6$ und $(S)-6$ zugänglich zu machen (Abbildung 16). Ein geeigneter Vorläufer zur Verwirklichung dieser stereodivergenten Synthesestrategie stellt der in hoher optischer Reinheit (ee > 95\%) erhältliche $(R)$-3Methylglutarsäurehalbester [(R)-11] dar. $(R)-11$ läßt sich nach bekannten Verfahren in die chiralen Lactone $(R)-\mathbf{1 2}$ und $(S)$-12 umwandeln. Aus ihnen sind dann die beiden Bausteine $(R)$-6 und (S)-6 zugänglich. Sie bilden die Grundlage für den Zugang zu den beiden Antipoden von Methanophenazin (1).

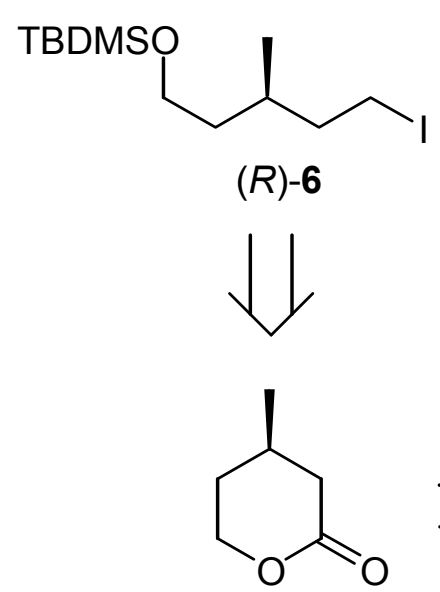

$(R)-12$

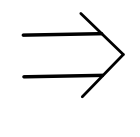

$\mathrm{HO}$<smiles>CCOC(=O)CC(C)CC(=O)O</smiles>

$(R)-11$<smiles>CS[OH+]CCC(C)CCI</smiles>

(S)-6<smiles>CCCC(C)C</smiles><smiles>CC(C)CC(C)C</smiles><smiles>CC1CCOC(=O)C1</smiles>

(S)-12

Abb. 16: Retrosyntheseschema der chiralen lodide $(R)-6$ und (S)-6 


\section{Strukturaufklärung von Methanophenazin (1)}

\subsection{Fermentation $^{[24]}$}

Die Anzucht von Methanosarcina mazei Gö1 im 1001 bzw. 3001 Fermenter wurde in einem für die Bedürfnisse methanogener Organismen optimierten Medium bei einem $\mathrm{pH}$ Wert von 6.9 bis 7.3 durchgeführt. Nach der Inocculation wurde der strikt anaerobe Organismus in einer $\mathrm{N}_{2} / \mathrm{CO}_{2}=4: 1$ Atmosphäre mit Methanol $(150 \mathrm{mM})$ als Substrat inkubiert. Eine Überprüfung des Substratverbrauchs durch gaschromatographische Untersuchungen des Mediums und Messungen der optischen Dichte bei $600 \mathrm{~nm}\left(\mathrm{OD}_{600}\right)$ zeigten das Ende der Inkubationszeit nach 2 bis 3 Tagen bei $37^{\circ} \mathrm{C}$ an.

Das Abtrennen der Zellen von der Kulturbrühe geschah in einer Durchlaufzentrifuge. Das Zellpellet wurde im unmittelbaren Anschluß an die Zentrifugation in flüssigem Stickstoff schockgefroren und bei $-70^{\circ} \mathrm{C}$ gelagert.

\subsection{Isolierung ${ }^{[24]}$}

Zur Isolierung der redoxaktiven Komponente aus Membranen von Methanosarcina mazei Gö1 wurden $100 \mathrm{~g}$ Zellen aus der Lagerung bei $-70{ }^{\circ} \mathrm{C}$ durch Auftauen in einem Morpholinopropansulfonsäure-Puffer (MOPS-Puffer) unter anaeroben Bedingungen aufgeschlossen. Die Zugabe des Enzyms DNase zum reduzierenden Medium gewährleistete die vollständige Zerkleinerung der polymeren DNA. Im ersten Schritt wurden durch niedertourige Zentrifugation $(8000 \mathrm{x}$ g) die noch intakten Zellen und Zellbruchstücke entfernt. Der Überstand wurde einer Ultrazentrifugation (120000 x g) unterzogen, um das Cytoplasma im Überstand von dem Membranpellet abzutrennen. Das Membranpellet wurde noch zweimal mit organischem MOPS-Puffer gewaschen und erneut ultrazentrifugiert. Die gewaschenen Membranen wurden in MOPS-Puffer suspendiert, in flüssigem Stickstoff eingefroren und über Nacht durch Gefriertrocknung vom Wasser befreit. Anschließend wurden die Membranen viermal mit je $30 \mathrm{ml}$ Isooctan (Merck, p.a. Qualität) extrahiert. Dazu wurden sie in Isooctan aufgenommen, mechanisch homogenisiert und der Überstand nach dem Sedimentieren der Membranen dekantiert. Die vereinigten Isooctanextrakte wurden abschließend zur Abtrennung der verbliebenen Membranen zentrifugiert und das Solvens unter den Bedingungen der Gefriertrocknung entfernt. Der erhaltene braun-gelbe Rohextrakt 
wurde bei $-20{ }^{\circ} \mathrm{C}$ gelagert. Aus $100 \mathrm{~g}$ Zellen wurden auf diesem Weg etwa $30 \mathrm{mg}$ Rohmaterial gewonnen. Insgesamt erhielt man aus 5 x 100 g Zellen 137 mg Rohextrakt.

\subsection{Reinigung}

$137 \mathrm{mg}$ Rohmaterial wurden in $10 \mathrm{ml}$ Isooctan gelöst und über einen Filter mit der Porengröße $0.45 \mu \mathrm{m} \quad$ (Sartorius, Membranfilter $\mathrm{RC}$ 15) filtriert. Ein Dünnschichtchromatogramm $($ Hexan/Ethylacetat $=10: 1)$ des Filtrats zeigte bei Tageslicht neben einem Startfleck den Spot des Phenazinethers 1 bei einem $\mathrm{R}_{\mathrm{f}}$-Wert von 0.51 . Untersuchte man das Chromatogramm im UV-Licht bei $\lambda=254 \mathrm{~nm}$, so detektierte man zusätzlich einige geringe Verunreinigungen im Bereich von $\mathrm{R}_{\mathrm{f}}=0.05$ bis 0.1 und einen $\mathrm{UV}$ löschenden Fleck bei $\mathrm{R}_{\mathrm{f}}=0.65$. Dieser konnte Dioctylphthalat (13) zugeordnet werden.

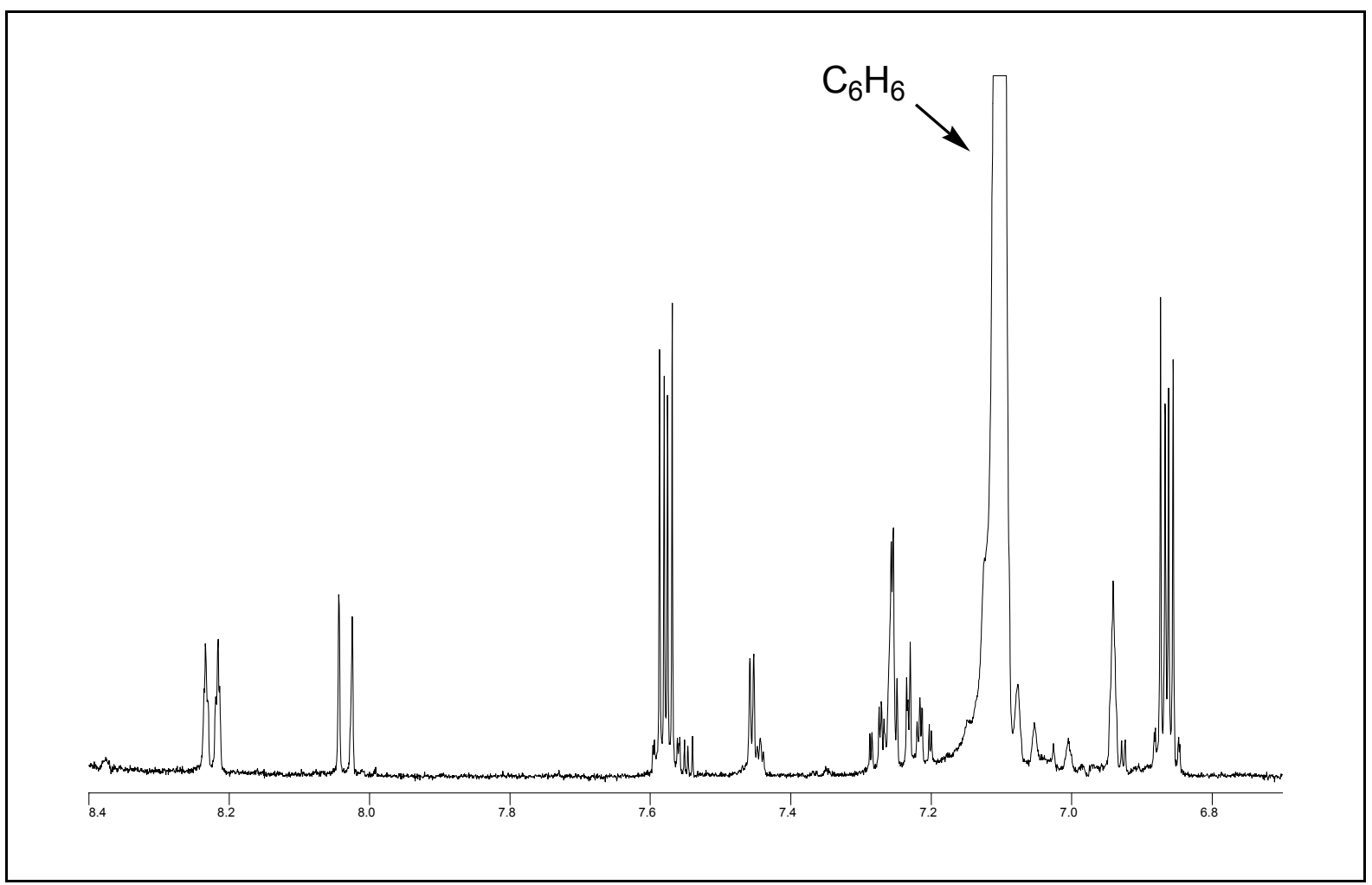

Abb. 17: Ausschnitt aus dem ${ }^{1} \mathrm{H}$ NMR-Spektrum eines Rohextrakts aus Membranen von Ms. mazei Gö1, abgebildet ist nur der Aromatenbereich (500 MHz, $\left.\mathrm{C}_{6} \mathrm{D}_{6}\right)$

Die in Abbildung 17 dargestellte Ausschnittsvergrößerung des ${ }^{1} \mathrm{H}$ NMR-Spektrums des Rohextrakts zeigt neben den charakteristischen Resonanzen der Aromatenprotonen des Phenazinethers 1 bei $\delta=7.20$ bis 7.30, 7.55, 8.04 und 8.23 ppm, weitere Signale bei $\delta=6.85$ 
und $7.59 \mathrm{ppm}$. Sie werden durch die aromatischen Protonen des Phthalsäureesters 13 hervorgerufen und sind anthropogenen Ursprungs.<smiles>CCCCCCCCCCOC(=O)c1ccccc1C(=O)OCCCC</smiles>

Die als Weichmacher in Polymeren zum Einsatz kommenden langkettigen Phthalsäureester sind ubiquitär verbreitet und finden sich regelmäßig bei der Analyse unpolarer Rückstände unterschiedlichster Herkunft. ${ }^{[32]}$ Der aromatische Kern ermöglicht eine Detektion im UVLicht bei $\lambda=260 \mathrm{~nm}$. Nach der Abtrennung vom Substanzgemisch durch HPLC wurde die Struktur des Phthalsäureesters 13 durch massenspektrometrische Untersuchungen, ${ }^{1} \mathrm{H}$ NMRSpektroskopie und Spektrenvergleich mit Literaturdaten ermittelt. ${ }^{[33]}$

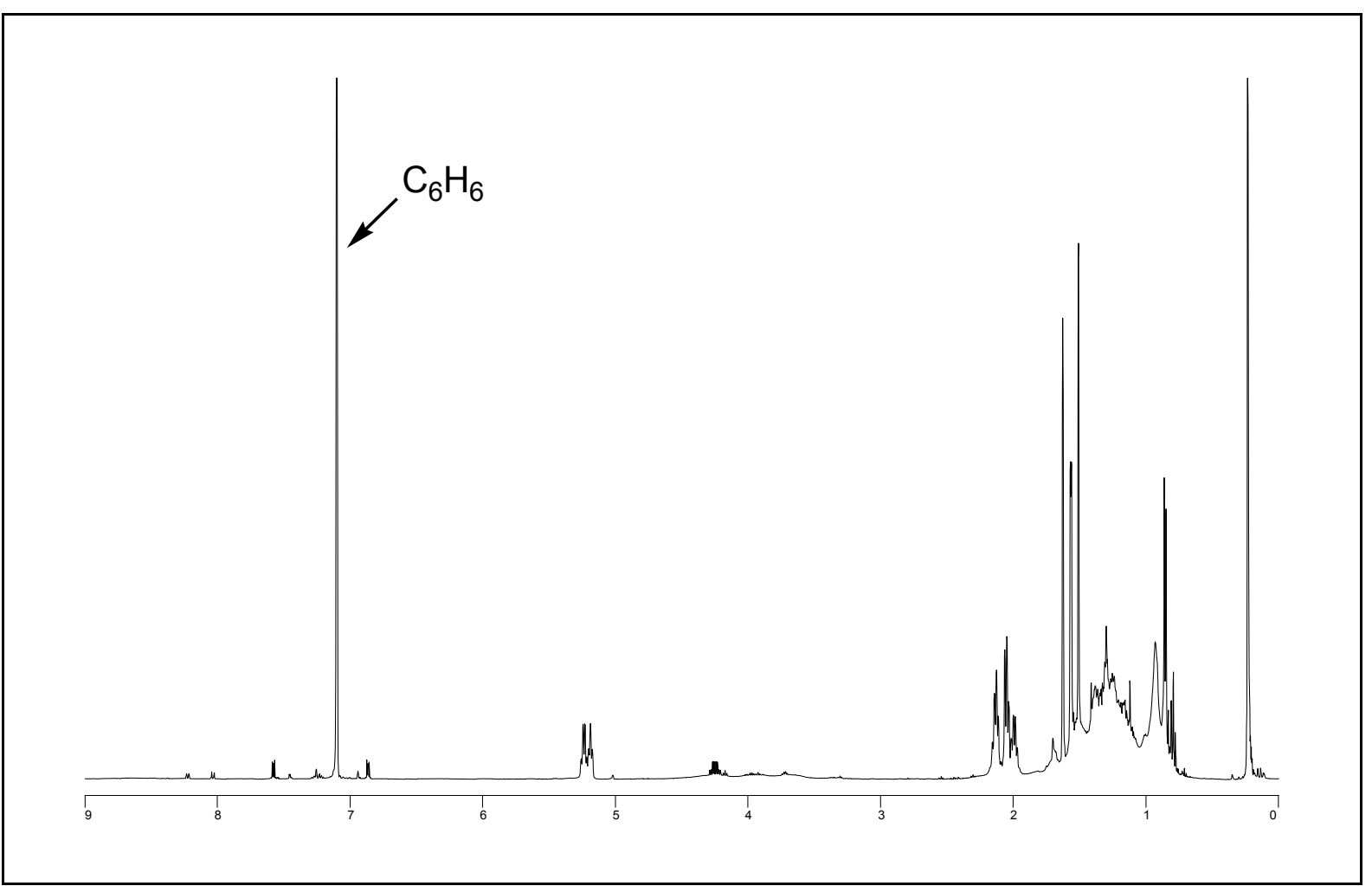

Abb. 18: ${ }^{1} \mathrm{H}$ NMR-Spektrum eines Rohextrakts aus Membranen von Methanosarcina mazei Gö1, $\left(500 \mathrm{MHz}, \mathrm{C}_{6} \mathrm{D}_{6}\right)$ 
Die Cytoplasmamembran von Archaebakterien unterscheidet sich wesentlich von der aller anderen Bakterien. Sie besitzt anstatt der bei Eubakterien vorkommenden Glycerinester Glycerinether mit isoprenoiden $\mathrm{C}_{20^{-}}$und $\mathrm{C}_{40^{-}}$-Alkylketten. Daneben enthält sie auch freie isoprenoide $\mathrm{C}_{15^{-}}$und $\mathrm{C}_{30}$-Kohlenwasserstoffe als neutrale Lipide. ${ }^{[10]}$ Die beschriebene Extraktionsmethode mit Isooctan als Extraktionsmittel ist unselektiv und löste alle unpolaren Komponenten aus der Cytoplasmamembran. Das in Abbildung 18 dargestellte ${ }^{1} \mathrm{H}$ NMRSpektrum des Rohmaterials zeigt im Bereich von $\delta=0.80$ bis 1.80 ppm starke Resonanz von Protonen, die große Mengen gesättigter Alkylverbindungen anzeigen. Ihre chromatographische Abtrennung von $\mathbf{1}$ bereitete erhebliche Schwierigkeiten, weil isoprenoide Alkyketten und Glycerinether sich hierbei ähnlich unpolar wie der Phenazinether 1 selbst verhielten. So werden bei der chromatographischen Aufreinigung des Rohextraktes durch HPLC-Techniken unter UV-Detektion Verunreinigungen wie beispielsweise Kohlenwasserstoffe, die über keinen Chromophor verfügen, nicht detektiert. Sie absorbieren im Bereich $\lambda \leq 190 \mathrm{~nm}$ und fallen in den ,cut off“-Bereich des Eluenten, werden also von dessen Absorption überlagert und können weder detektiert noch gezielt abgetrennt werden.

Um eine gute Auftrennung der Komponenten zu erreichen, wurden erste Versuche mit einer „reversed phase“-HPLC-Säule (LiChrospher RP 18, 4 x 125 mm, Merck) durchgeführt. Bei diesem Typ von stationärer Phase wird die Kieselgeloberfläche mit Alkylketten unterschiedlicher Länge $\left(\mathrm{C}_{8}-\mathrm{C}_{18}\right)$ modifiziert, wodurch sich ihre Polarität umkehrt. Unpolare Substanzgemische treten nun in starke Wechselwirkung mit der unpolaren stationären Phase. Dies bewirkt eine, im Vergleich mit der reinen Kieselgelphase, verbesserte Trennleistung der stationären Phase und führt zu einer besseren Separation der einzelnen Komponenten des Gemischs. Bei dieser Art von Säulenmaterial haben sich Methanol und Acetonitril, oft in Verbindung mit unterschiedlichen Anteilen Wasser, als Eluenten bewährt. ${ }^{[34]}$

Die Ergebnisse, die bei Anwendung dieser Trennmethode zur Aufreinigung des Rohextrakts aus Membranen von Ms. mazei Gö1 erzielt wurden, waren nicht zufriedenstellend. Zwar gelang es, nahezu alle Verunreinigungen abzutrennen, dennoch enthielten alle ${ }^{1} \mathrm{H}$ NMRSpektren neben den Resonanzen des Phenazinethers 1 noch Signale einer Verunreinigung im Bereich zwischen $\delta=1.0$ bis $1.6 \mathrm{ppm}$, die sich durch eine breite unaufgelöste Bande zu erkennen gab, und die auch durch mehrmaliges Chromatographieren derselben Substanzprobe nicht abzutrennen war. Massenspektrometrische Untersuchungen der Substanzprobe und die Durchführung von Blindversuchen mit anschließender Analyse der dabei angefallenen Rückstände führten zur Identifikation der Kontamination als Bestandteil der RP-Phase $\left(\mathrm{C}_{18}\right)$, 
die aus der HPLC-Säule durch den Eluenten Methanol herausgelöst worden war. Da es sich bei dem isolierten Methanophenazin (1) in der Regel um weniger als $1 \mathrm{mg}$ an aufgereinigter Substanz handelte, führten auch geringe Verunreinigung an RP-Material $(\leq 0.1 \mathrm{mg}) \mathrm{zu}$ störenden Signalen in den ${ }^{1} \mathrm{H}$ NMR-Spektren, so daß auf eine weitere Chromatographie an dieser Art von stationärer Phase verzichtet wurde.

Da der Bereich von $\delta=1.0$ bis $1.6 \mathrm{ppm}$ des ${ }^{1} \mathrm{H}$ NMR-Spektrums von entscheidender Bedeutung zur Aufklärung der Struktur der Seitenkette von 1 war, wurden andere Trennbedingungen zur Aufreinigung des Rohmaterials ausgearbeitet (Abbildung 19).

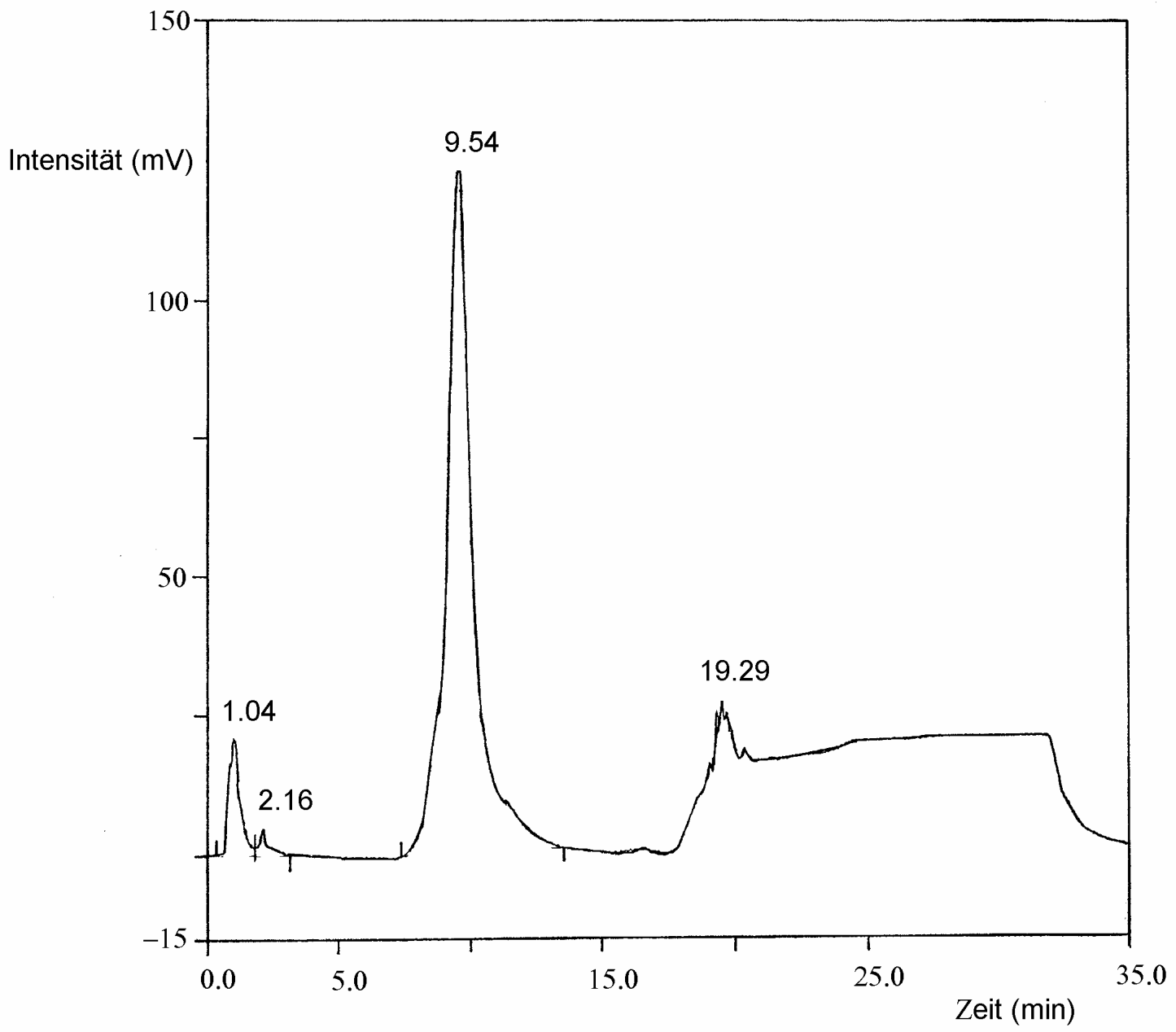

Abb. 19: Analytisches HPLC-Chromatogramm des Rohextrakts aus Membranen von Ms. mazei Gö1 (Kieselgelsäule: LiChrospher Si 60, 4 x 125 mm, Merck; Flußrate: 1.0 ml/min; UVDetektion: $\lambda=260 \mathrm{~nm}$; Eluent A: Cyclohexan, Eluent B: Ethylacetat; Eluentengradient: 0 bis $15 \min 3 \%$ B; 15 bis 25 min stufenloser Gradient bis $100 \%$ B; 25 bis 30 min $100 \%$ B, 30 bis $35 \min 3 \% \mathrm{~B}$ ) 
Eine Separation des Naturstoffs von Verunreinigungen gelang durch Chromatographie an einer analytischen Kieselgelsäule (LiChrospher Si 60, 4 x 125 mm, Merck). Sie ergab das in Abbildung 19 dargestellte Chromatogramm. Es zeigt neben einigen Verunreinigungen während der ersten beiden und nach 15 min den Hauptpeak, der durch die Absorption von 1 nach einer Retentionszeit von 9.54 min erzeugt wurde. Der Anteil an Essigsäureethylester (Eluent B) im Laufmittel wurde nach 15 min kontinuierlich von 3 auf $100 \%$ gesteigert. Dies hat einen Anstieg der Grundlinie nach ca. 17.5 min, hervorgerufen durch die Absorption dieses Eluenten, zur Folge. Eine Verminderung des Anteils Ethylacetat am Eluentengemisch läßt die Grundlinie wieder auf ihren ursprünglichen Wert fallen, was bei einer Retentionszeit von ca. 32 min zu erkennen ist.

Die Isolierung des Naturstoffs 1 wurde durch halbpräparative HPLC unter folgenden Bedingungen durchgeführt: semipräparative Kieselgelsäule (Si 60, 10 x 250 mm, Kontron); Flußrate: $4.0 \mathrm{ml} / \mathrm{min}$; Detektion: $\lambda=260 \mathrm{~nm}$; Eluent A: Cyclohexan; Eluent B: Ethylacetat; Eluentengradient: 0 bis $15 \min 5 \% \mathrm{~B}, 15$ bis $22 \min 30 \% \mathrm{~B}, 22$ bis 28 min stufenloser Gradient bis $100 \%$ B, 28 bis $35 \mathrm{~min} 5 \% \mathrm{~B}$. Dabei wurden pro Lauf etwa $10 \mathrm{mg}$ Substanzgemisch in $200 \mu 1$ Isooctan auf die Säule aufgetragen.

Nach der chromatographischen Reinigung von 137 mg Rohextrakt aus Cytoplasmamembranen von 5 x $100 \mathrm{~g}$ Zellen von Methanosarcina mazei Gö1, wurden $1.2 \mathrm{mg}$ reines Methanophenazin (1) als gelbes Öl isoliert (Abbildung 20). 
Anzucht von Ms. mazei Gö1 im 100 I Fermenter: 150 mM Methanol, pH 6.9 -7.3, anaerobe

Bedingungen in $\mathrm{N}_{2} / \mathrm{CO}_{2}$-Atmosphäre, $2-3 \mathrm{~d}, 37^{\circ} \mathrm{C}$

$\Longrightarrow$ Zellmasse: $\approx 100 \mathrm{~g}$<smiles>CCCCC</smiles>

Membranpräparation: Abtrennen der Cytoplasmamembranen von Zellwand, DNA und anderen Zellbestandteilen durch Zentrifugation; Isooctanextraktion der Cytoplasmamembranen

$\Longrightarrow$ Rohextrakt: $\approx 30 \mathrm{mg}$<smiles>CCCCC</smiles>

HPLC-Reinigung des Rohextrakts:

$\mathrm{SiO}_{2}$-Säule; Gradient: Cyclohexan/Essigester, Detektion: $\lambda=260 \mathrm{~nm}$

$\Rightarrow$ Reinsubstanz: $\approx 0.3 \mathrm{mg}$<smiles>CCCCC</smiles>

\section{Strukturaufklärung:}

Massenspektrometrie, ${ }^{1} \mathrm{H}$ - und ${ }^{13} \mathrm{C}$ NMR-Spektroskopie, zweidimensionale NMR-Korrelationsexperimente<smiles>CCCCC</smiles><smiles>CC(C)=CCC/C(C)=C/CC/C(C)=C/CC/C(C)=C/CC[C@@H](C)CCOc1ccc2nc3ccccc3nc2c1</smiles>

Methanophenazin (1)

das erste Phenazinderivat aus Archaea

Abb. 20: Schema zur Isolierung, Reinigung und Strukturaufklärung von Methanophenazin (1) aus Cytoplasmamembranen von Methanosarcina mazei Gö1 


\subsection{Strukturaufklärung}

Die Summenformel des Naturstoffs 1, von dem insgesamt nur 1.2 mg zur Verfügung standen, wurde durch hochauflösende Massenspektrometrie bestimmt. Seine Konstitution und die Struktur der Seitenkette wurde durch ein- und zweidimensionale ${ }^{1} \mathrm{H}-$ und ${ }^{13} \mathrm{C}$ Kernresonanzexperimente (HMQC, H,H COSY, HMBC) ermittelt.

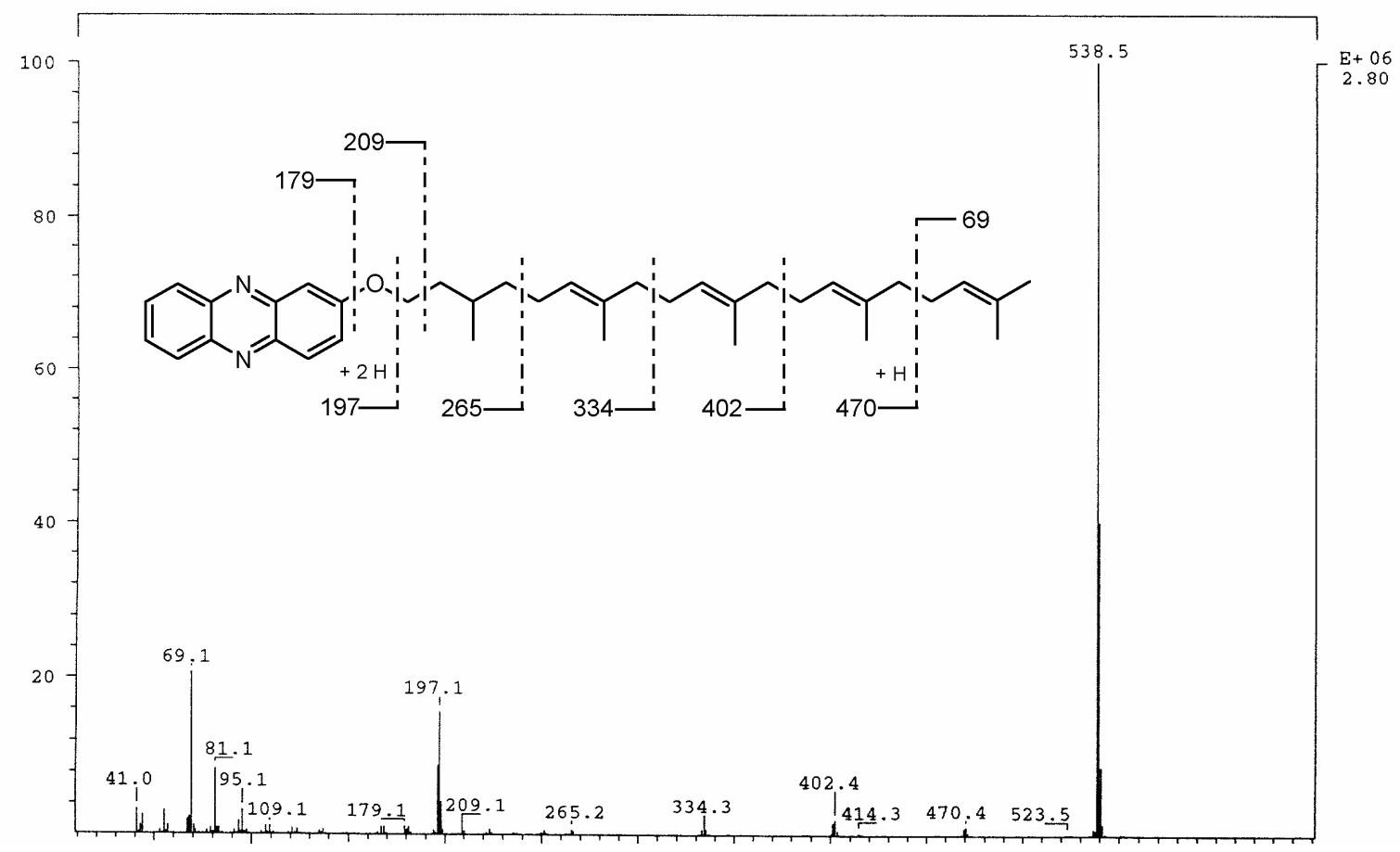

Abb. 21: El (70 eV) Massenspektrum von Methanophenazin (1)

Das EI Massenspektrum präsentiert das Molekülion bei $m / z=538$ als Basispeak (Abbildung 21). Das Zerfallsmuster zeigt Fragmente mit geringer Intensität $(<4 \%)$ bei $m / z=470,402$, 334 und 265. Die Differenzen zu $\mathrm{M}^{+}$und untereinander betragen $m / z=68$ bzw. 69 Masseneinheiten und entsprechen dem sukzessiven Verlust von $\mathrm{C}_{5} \mathrm{H}_{8}$ bzw. $\mathrm{C}_{5} \mathrm{H}_{9}$ durch Allylspaltung. Dieses Verhalten unter Elektronenbeschuß ist charakteristisch für Verbindungen mit linearen, isoprenoiden Ketten und wird beispielsweise bei Geranylgeranylbenzochinonen beobachtet. ${ }^{[35]}$ Eine Hochauflösung des Molekülions (EI-HRMS, ber.: 538.3923, gef.: 538.3930) zur Bestimmung der Elementarzusammensetzung ergab für $\mathbf{1} \mathrm{C}_{37} \mathrm{H}_{50} \mathrm{~N}_{2} \mathrm{O}$ als Summenformel. Hieraus läßt sich aufgrund der Valenzen der einzelnen Elemente die Gesamtzahl der Ringe und Doppelbindungen einer Verbindung bestimmen. Für Molekülionen mit ungerader Elektronenzahl und der Summenformel $\mathrm{C}_{\mathrm{x}} \mathrm{H}_{\mathrm{y}} \mathrm{N}_{\mathrm{z}} \mathrm{O}_{\mathrm{n}}$ beträgt sie $\mathrm{x}-(\mathrm{y} / 2)+(\mathrm{z} / 2)$ 
+ 1. Die Elementarzusammensetzung von Methanophenazin (1) $\mathrm{C}_{37} \mathrm{H}_{50} \mathrm{~N}_{2} \mathrm{O}$ deutet demnach auf 14 Doppelbindungsäquivalente hin. ${ }^{[36]}$

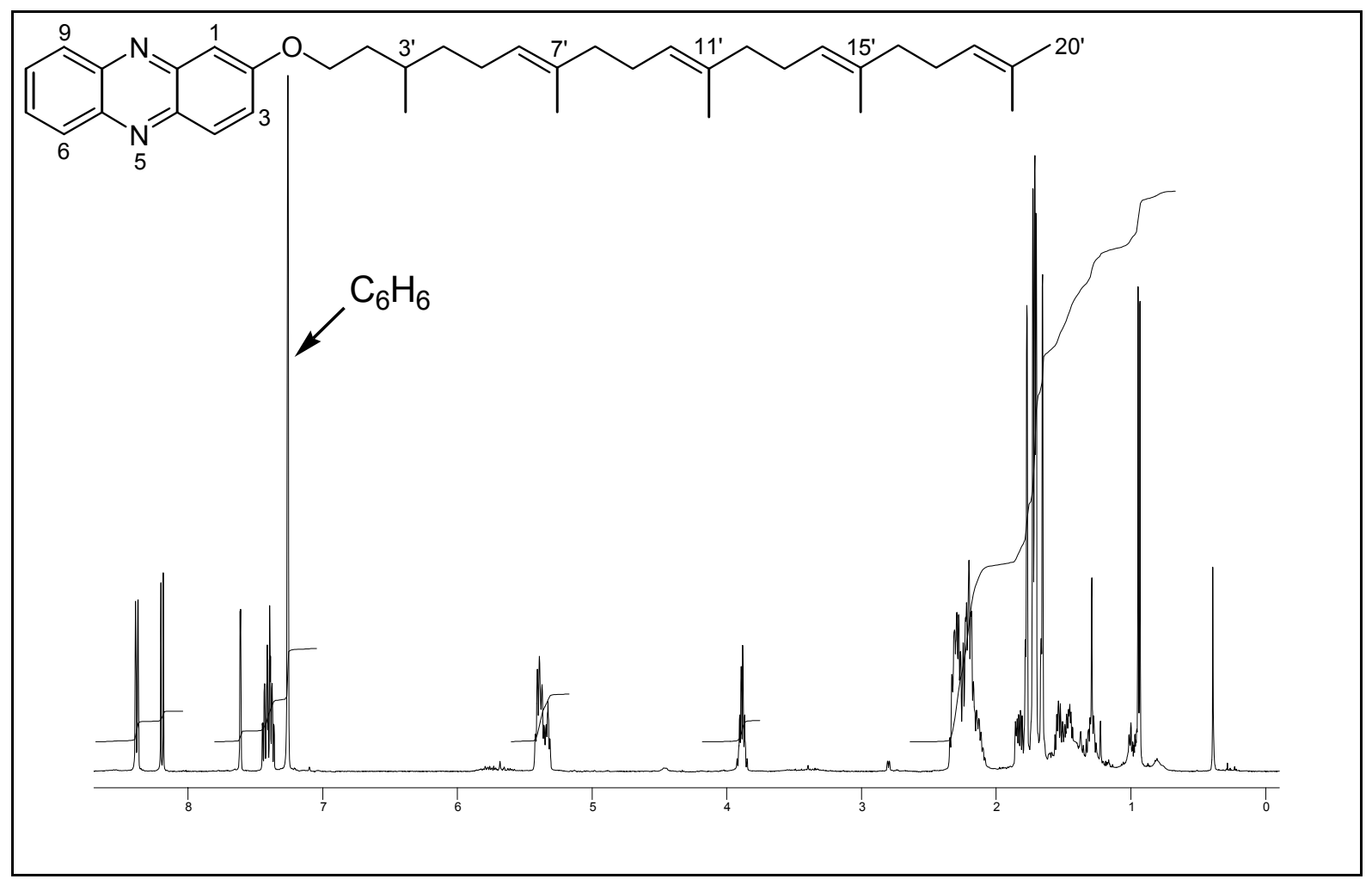

Abb. 22: ${ }^{1} \mathrm{H}$ NMR-Spektrum von natürlichem Methanophenazin (1) (500 MHz, $\left.\mathrm{C}_{6} \mathrm{D}_{6}\right)$

Das ${ }^{1} \mathrm{H}$ NMR-Spektrum von 1 in Deuterobenzol lassen sich bei $\delta=7.30,8.12,8.30$ und $8.31 \mathrm{ppm}$ die Resonanzen von sieben aromatischen Protonen erkennen (Abbildung 22). Die Signale bei $\delta=5.25$ bis 5.38 ppm werden vier olefinischen Protonen zugeordnet, während die Resonanz einer Methylengruppe bei $\delta=3.80 \mathrm{ppm}$ durch die unmittelbare Nähe eines Sauerstoffatoms charakteristisch zu tiefem Feld verschoben wird. Die verbleibenden 37 Protonen resonieren im aliphatischen Bereich zwischen $\delta=0.85$ bis 2.20 ppm und komplizieren die Analyse des Spektrums durch zahlreiche sich überlagernde Signale. Beispielsweise zeigen 14 allylische Protonen bei $\delta=1.97$ bis $2.27 \mathrm{ppm}$ ein nicht aufgelöstes Multiplett, hingegen lassen sich 15 an Doppelbindungen fixierte Methylprotonen in Gestalt von fünf Singuletts bei $\delta=1.56$ bis $1.68 \mathrm{ppm}$ deutlich erkennen. Sie überlagern hier ein Methinproton, das durch die genaue Auswertung des Integrals über diesen Bereich nachgewiesen wurde. Gut $\mathrm{zu}$ analysieren ist das Dublett einer Methylgruppe bei $\delta=0.83$ ppm, was auf ein tertiäres C-Atom in $\alpha$-Position deutet und ein stereogenes Zentrum anzeigt. 
Der Bereich von $\delta=1.14$ bis 1.44 ppm beherbergt noch vier aliphatische Protonen, die der gesättigten Teilstruktur des Naturstoffs zugeordnet werden. Die ${ }^{1} \mathrm{H}$ NMR-Daten von $\mathbf{1}$ in Deuterobenzol sind in Tabelle 2 zusammengefaßt.

Tabelle 2: ${ }^{1} \mathrm{H}$ NMR-Daten von Methanophenazin (1) $\left(500 \mathrm{MHz}, \mathrm{C}_{6} \mathrm{D}_{6}\right)$

\begin{tabular}{|c|c|c|c|}
\hline Protonen & $\delta_{\mathbf{C}_{6} \mathbf{D}_{6}}[\mathrm{ppm}]$ & Multiplizität, $J$ in $[\mathrm{Hz}]$ & $\begin{array}{c}\text { Anzahl der } \\
\text { Protonen }\end{array}$ \\
\hline $1-\mathrm{H}$ & 7.48 & $\mathrm{~d}(2.7)$ & $1 \mathrm{H}$ \\
\hline $3-\mathrm{H}, 7-\mathrm{H}, 8-\mathrm{H}$ & $7.22-7.33$ & $\mathrm{~m}$ & $3 \mathrm{H}$ \\
\hline $4-\mathrm{H}$ & 8.06 & $\mathrm{~d}(9.4)$ & $1 \mathrm{H}$ \\
\hline $6-\mathrm{H}$ oder $9-\mathrm{H}$ & 8.24 & $\mathrm{dd}(8.8,1.2)$ & $1 \mathrm{H}$ \\
\hline 9-H oder $6-\mathrm{H}$ & 8.245 & $\mathrm{dd}(8.8,1.2)$ & $1 \mathrm{H}$ \\
\hline $1 '-\mathrm{H}_{2}$ & 3.76 & $\mathrm{~m}_{\mathrm{C}}$ & $2 \mathrm{H}$ \\
\hline $2^{\prime}-\mathrm{H}_{\mathrm{A}}$ & 1.69 & $\mathrm{~m}_{\mathrm{C}}$ & $1 \mathrm{H}$ \\
\hline $2^{\prime}-\mathrm{H}_{\mathrm{B}}, 4^{\prime}-\mathrm{H}_{\mathrm{A}}$ & $1.30-1.44$ & $\mathrm{~m}$ & $2 \mathrm{H}$ \\
\hline $3 '-H$ & $1.54-1.63$ & $\mathrm{~m}$ & $1 \mathrm{H}$ \\
\hline $3^{\prime}-\mathrm{CH}_{3}$ & 0.81 & $d(6.6)$ & $3 \mathrm{H}$ \\
\hline $4^{\prime}-\mathrm{H}_{\mathrm{B}}$ & 1.17 & $\mathrm{~m}_{\mathrm{C}}$ & $1 \mathrm{H}$ \\
\hline $\begin{array}{c}5^{\prime}-\mathrm{H}_{2}, 8^{\prime}-\mathrm{H}_{2}, 9^{\prime}-\mathrm{H}_{2}, 12^{\prime}-\mathrm{H}_{2}, \\
13^{\prime}-\mathrm{H}_{2}, 16^{\prime}-\mathrm{H}_{2}, 17^{\prime}-\mathrm{H}_{2},\end{array}$ & $1.94-2.23$ & $\mathrm{~m}$ & $14 \mathrm{H}$ \\
\hline $6^{\prime}-\mathrm{H}, 10^{\prime}-\mathrm{H}, 14^{\prime}-\mathrm{H}$ & $5.22-5.32$ & $\mathrm{~m}$ & $3 \mathrm{H}$ \\
\hline $18^{\prime}-\mathrm{H}$ & 5.20 & t br (6.9) & $1 \mathrm{H}$ \\
\hline 7- $\mathrm{CH}_{3}, 11^{\prime}-\mathrm{CH}_{3}, 15^{\prime}-\mathrm{CH}_{3}$ & $1.58,1.59,1.60$ & $3 \mathrm{~s}$ & je $3 \mathrm{H}$ \\
\hline $19^{\prime}-\mathrm{CH}_{3}$ & 1.53 & $\mathrm{~s}$ & $3 \mathrm{H}$ \\
\hline $20^{\prime}-\mathrm{H}_{3}$ & 1.63 & $\mathrm{~d}(1.0)$ & $3 \mathrm{H}$ \\
\hline
\end{tabular}




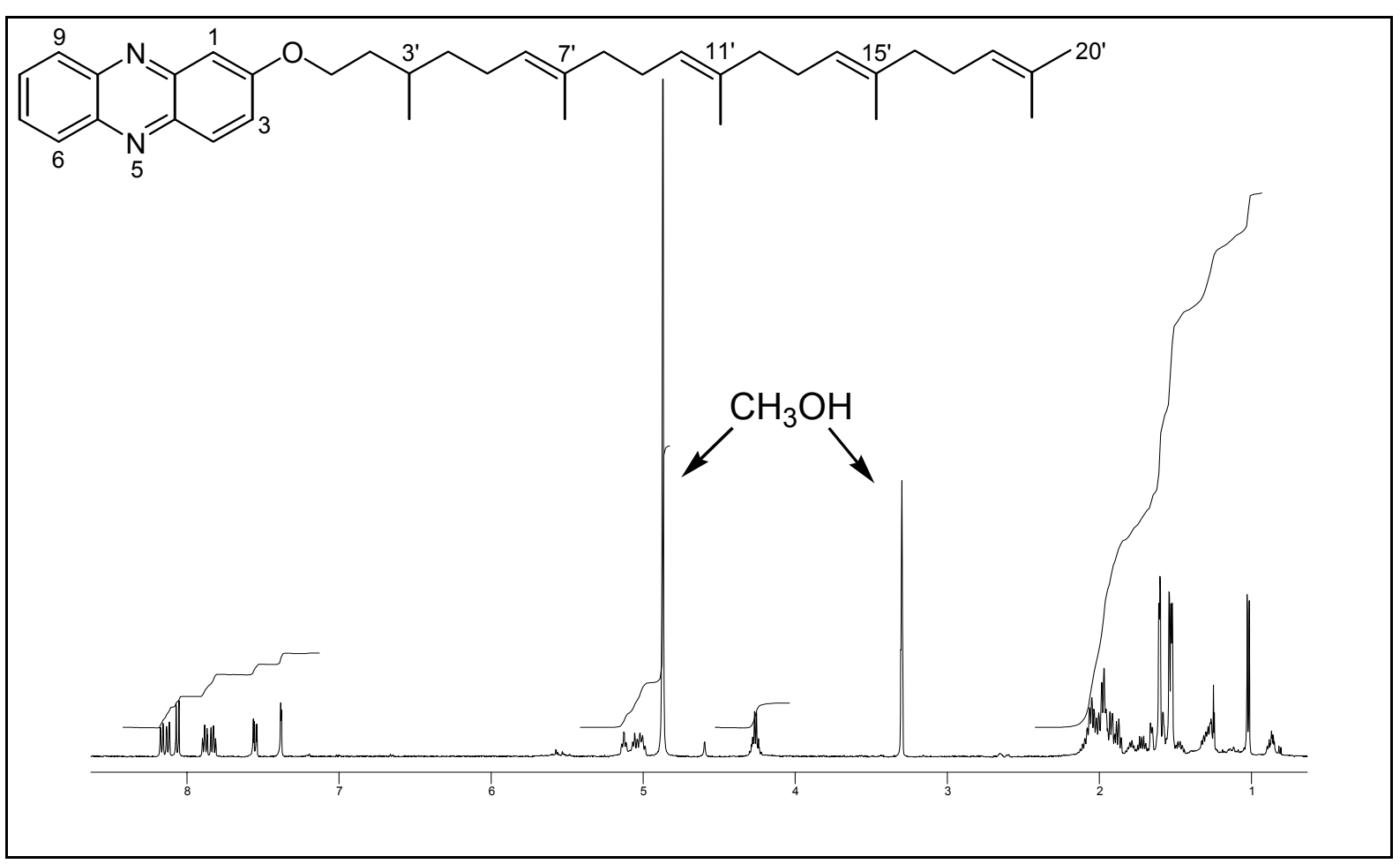

Abb. 23: ${ }^{1} \mathrm{H}$ NMR-Spektrum von natürlichem Methanophenazin (1) (500 MHz, $\mathrm{CD}_{3} \mathrm{OD}$ )

Eine Aufnahme in Deuteromethanol (Abbildung 23) hat im aliphatischen und allylischen Bereich des Protonenspektrums nur wenig Einfluß auf die Signallage und Multiplizität. Im Bereich des Aromaten hingegen löst sich das Multiplett von 3 Protonen bei $\delta_{\mathrm{C}_{6} \mathrm{D}_{6}}=7.30 \mathrm{ppm}$ auf, und die Signale erscheinen separiert bei $\delta_{\mathrm{CD}_{3} \mathrm{OD}}=7.55,7.83$ und 7.89 ppm (Abbildung 25). Das Dublett vom Dublett von zwei Protonen bei $\delta_{\mathrm{C}_{6} \mathrm{D}_{6}}=8.31 \mathrm{ppm}$ wird zu zwei Dubletts vom Dublett bei $\delta_{\mathrm{CD}_{3} \mathrm{OD}}=8.13$ und 8.17 ppm aufgelöst. Insgesamt können die sieben aromatischen Protonen nun sieben separaten Resonanzen zugeordnet werden. Eine detaillierte Analyse der Kopplungsmuster und Kopplungskonstanten dieser Signale führt zu zwei aromatischen Teilstrukturen mit unterschiedlichen Substitutionsmustern. Eine Teilstruktur mit den drei Protonen 1-H, 3-H und 4-H bei $\delta=7.38,7.55$ und 8.07 ppm bildet ein $\mathrm{ABC}$ Spinsystem aus. Die andere Teilstruktur mit den vier Protonen 6-H, 7-H, 8-H und 9-H bei $\delta=$ 7.83, 7.89, 8.13 und 8.17 ppm erzeugt ein ABCD Spinsystem (Abbildung 24). 
<smiles>CC(C)(C)O[Mg]</smiles>

Abb. 24: Spinsysteme und Kopplungskonstanten des Aromatenbereichs von Methanophenazin (1) $\left(500 \mathrm{MHz}, \mathrm{CH}_{3} \mathrm{OD}\right)$

Wechselwirkungen der Spinsysteme untereinander, über die Stickstoffatome hinweg, werden nicht beobachtet. Die deutliche Verschiebung der aromatischen Protonen 4-H, 6-H und 9-H mit $\delta=8.07,8.13$ und $8.17 \mathrm{ppm}$ zu tiefem Feld zeigt den negativen induktiven Effekt der benachbarten Stickstoffatome an. Die ${ }^{1} \mathrm{H}$ NMR-Daten von 1 in Deuteromethanol sind in Tabelle 3 zusammengefaßt.

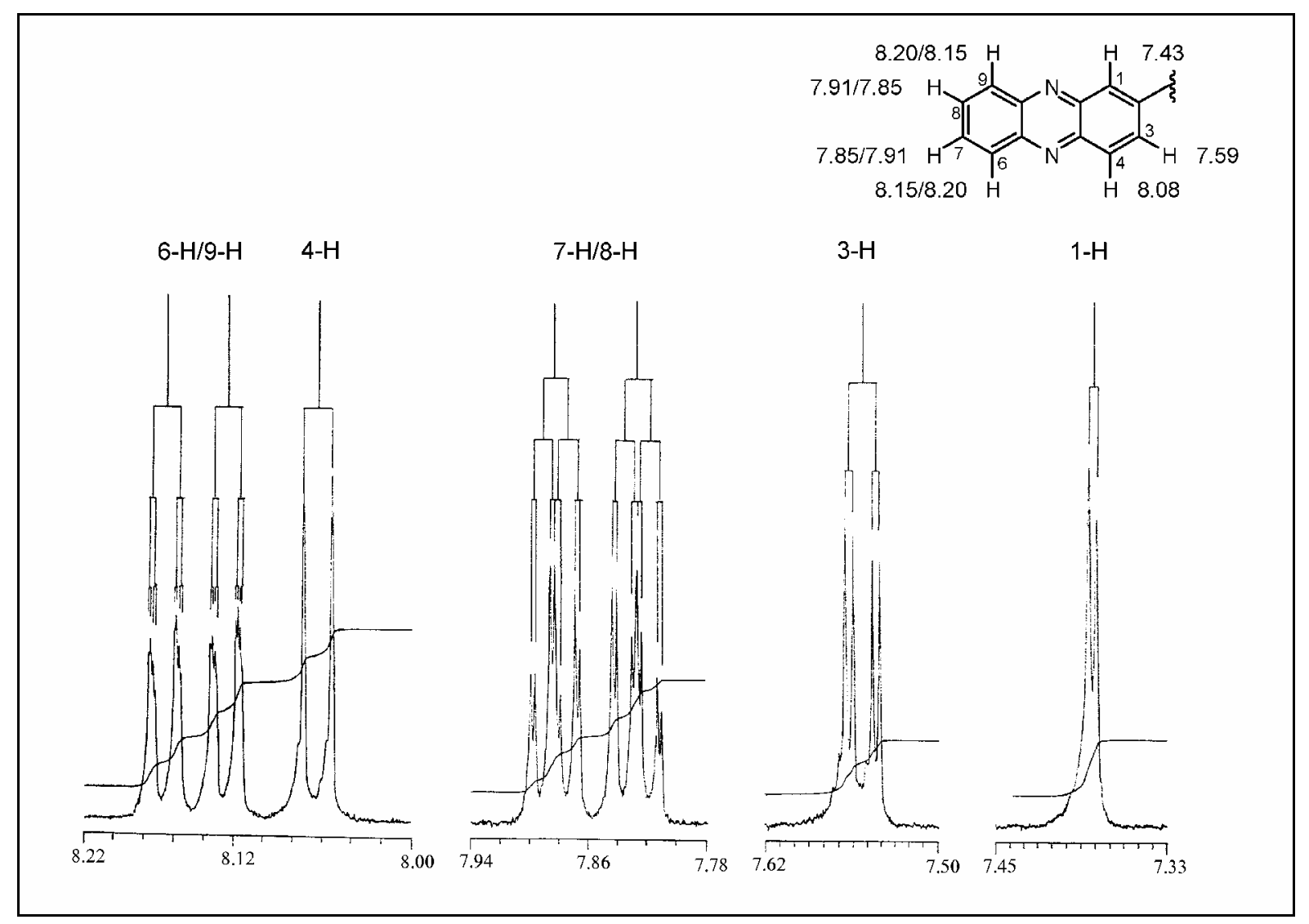

Abb. 25: ${ }^{1} \mathrm{H}$ NMR-Spektrum des Aromatenbereichs von Methanophenazin (1) $\left(500 \mathrm{MHz}, \mathrm{CD}_{3} \mathrm{OD}\right)$ 
Tabelle 3: ${ }^{1} \mathrm{H}$ NMR-Daten von Methanophenazin (1) $\left(500 \mathrm{MHz}, \mathrm{CD}_{3} \mathrm{OD}\right)$

\begin{tabular}{|c|c|c|c|}
\hline Protonen & $\delta_{\mathrm{CD}_{3} \mathrm{OD}}[\mathrm{ppm}]$ & Multiplizität, $J$ in $[\mathrm{Hz}]$ & $\begin{array}{c}\text { Anzahl der } \\
\text { Protonen }\end{array}$ \\
\hline $1-\mathrm{H}$ & 7.42 & $\mathrm{~d}(2.4)$ & $1 \mathrm{H}$ \\
\hline $3-\mathrm{H}$ & 7.58 & $\mathrm{dd}(9.4,2.5)$ & $1 \mathrm{H}$ \\
\hline $4-\mathrm{H}$ & 8.09 & $\mathrm{~d}(9.4)$ & $1 \mathrm{H}$ \\
\hline $6-\mathrm{H}$ oder $9-\mathrm{H}$ & 8.19 & ddd $(8.5,1.4,0.6)$ & $1 \mathrm{H}$ \\
\hline 7-H oder 8 -H & 7.85 & ddd $(8.4,6.7,1.4)$ & $1 \mathrm{H}$ \\
\hline 8-H oder $7-\mathrm{H}$ & 7.91 & ddd $(8.4,6.7,1.5)$ & $1 \mathrm{H}$ \\
\hline 9-H oder 6-H & 8.15 & ddd $(8.8,0.8,0.6)$ & $1 \mathrm{H}$ \\
\hline $1^{\prime}-\mathrm{H}_{2}$ & 4.29 & $\mathrm{~m}_{\mathrm{C}}$ & $2 \mathrm{H}$ \\
\hline $2^{\prime}-\mathrm{H}_{\mathrm{A}}$ & $1.79-1.86$ & $\mathrm{~m}$ & $1 \mathrm{H}$ \\
\hline $2^{\prime}-\mathrm{H}_{\mathrm{B}}$ & $1.70-1.79$ & $\mathrm{~m}$ & $1 \mathrm{H}$ \\
\hline $3^{\prime}-\mathrm{H}$ & $1.46-1.53$ & $\mathrm{~m}$ & $1 \mathrm{H}$ \\
\hline $3 '-\mathrm{CH}_{3}$ & 1.04 & $d(6.6)$ & $3 \mathrm{H}$ \\
\hline $4^{\prime}-\mathrm{H}_{2}$ & $1.27-1.37$ & $\mathrm{~m}$ & $2 \mathrm{H}$ \\
\hline $\begin{array}{c}5^{\prime}-\mathrm{H}_{2}, 8^{\prime}-\mathrm{H}_{2}, 9^{\prime}-\mathrm{H}_{2}, 12^{\prime}-\mathrm{H}_{2} \\
13^{\prime}-\mathrm{H}_{2}, 16^{\prime}-\mathrm{H}_{2}, 17^{\prime} \mathrm{H}_{2}\end{array}$ & $1.87-2.17$ & $\mathrm{~m}$ & $14 \mathrm{H}$ \\
\hline $6^{\prime}-\mathrm{H}$ & 5.08 & t br (7.9) & $1 \mathrm{H}$ \\
\hline $10^{\prime}-\mathrm{H}, 14^{\prime}-\mathrm{H}$, & $4.99-5.06$ & $\mathrm{~m}$ & $2 \mathrm{H}$ \\
\hline $18^{\prime}-\mathrm{H}$ & 5.15 & $\mathrm{t}$ br $(7.0)$ & $1 \mathrm{H}$ \\
\hline $7^{\prime}-\mathrm{CH}_{3}$ & 1.62 & $\mathrm{~s}$ & $3 \mathrm{H}$ \\
\hline $\begin{array}{c}11^{\prime}-\mathrm{CH}_{3}, \\
15^{\prime}-\mathrm{CH}_{3}, 19^{\prime}-\mathrm{CH}_{3}\end{array}$ & $1.54,1.55,1.56$ & $3 \mathrm{~s}$ & je $3 \mathrm{H}$ \\
\hline $20^{\prime}-\mathrm{H}_{3}$ & 1.63 & $\mathrm{~d}(1.0)$ & $3 \mathrm{H}$ \\
\hline
\end{tabular}

Die ${ }^{13} \mathrm{C}$ NMR- und ${ }^{13} \mathrm{C}$ APT-Spektren enthüllen neun quartäre C-Atome (Abbildung 26). Das C-2-Atom erfährt durch die direkte Verknüpfung mit dem Sauerstoff den größten Tieffeldshift und resoniert bei $\delta=160.78 \mathrm{ppm}$. Die Signale der vier quartären aromatischen Kohlenstoff-atome C-4a, C-5a, C-9a und C-10a finden sich im Bereich von $\delta=140$ bis 145 ppm. Drei olefinische quartäre C-Atome (C-7', C-11' und C-15') zeigen, ihrer ähnlichen chemischen Umgebung entsprechend, mit $\delta=135.09$, 135.04 und 134.99 nahezu identische chemische Verschiebungen. Das C-19' erfährt einen für quartäre, terminale Kohlenstoffe 
isoprenoider Natur charakteristischen leichten Hochfeldshift und resoniert bei $\delta=131.07$. Die ${ }^{13} \mathrm{C}$ NMR-Daten von Methanophenazin (1) sind in Tabelle 4 zusammengefaßt.

Tabelle 4: ${ }^{13} \mathrm{C}$ NMR-Daten von Methanophenazin (1) $\left(125 \mathrm{MHz}, \mathrm{APT}: \mathrm{-}^{\prime}=\mathrm{CH}_{2}, \mathrm{C}_{\text {quart }}\right.$ und ' $+{ }^{\prime}=\mathrm{CH}_{3}, \mathrm{CH}$ )

\begin{tabular}{|c|c|c|c|}
\hline Kohlenstoffatome & $\delta_{C_{6} D_{6}}[p p m]$ & $\delta_{\mathrm{CD}_{3} \mathrm{OD}}[\mathrm{ppm}]$ & APT \\
\hline $\mathrm{C}-1$ & 105.80 & 105.41 & ${ }^{\prime}+{ }^{\prime}$ \\
\hline $\mathrm{C}-2$ & 160.78 & 162.61 & ${ }^{\prime}-{ }^{\prime}$ \\
\hline $\mathrm{C}-3$ & 126.53 & 128.35 & ${ }^{\prime}+{ }^{\prime}$ \\
\hline C-4 & 131.29 & 131.31 & ${ }^{\prime}+{ }^{\prime}$ \\
\hline$C-4 a$ & 145.87 & 146.23 & ${ }^{-}-$ \\
\hline C-5a oder C-9a & 144.12 & 144.21 & ${ }^{-}{ }^{\prime}$ \\
\hline C-6 oder C-9 & 130.26 & 129.31 & ${ }^{\prime}+$ \\
\hline C-7 oder C-8 & 129.56 & 132.19 & ${ }^{\prime}+$ \\
\hline C-8 oder C-7 & 128.78 & 130.66 & ${ }^{\prime}+$ \\
\hline C-9 oder C-6 & 130.17 & 130.22 & ${ }^{\prime}+$ \\
\hline C-9a oder C-5a & 142.63 & 142.77 & ${ }^{-}$ \\
\hline C-10a & 141.36 & 141.78 & $-^{\prime}$ \\
\hline $\mathrm{C}-1$ & 66.91 & 68.36 & $-^{\prime}$ \\
\hline $\mathrm{C}-2^{\prime}$ & 36.01 & 36.96 & $-^{\prime}$ \\
\hline $\mathrm{C}-3$ & 29.80 & 30.58 & ${ }^{\prime}+$ \\
\hline 3'- $-\mathrm{CH}_{3}$ & 19.56 & 20.05 & $'+$ \\
\hline C-4' & 37.45 & 38.19 & ${ }^{\prime}$ \\
\hline $\mathrm{C}-5$ & 25.83 & 26.31 & ${ }^{-}{ }^{\prime}$ \\
\hline $\mathrm{C}-6^{\prime}, \mathrm{C}-10^{\prime}, \mathrm{C}-14^{\prime}$ & $124.77,124.93,124.73$ & $125.43,125.45$ & $+^{\prime}$ \\
\hline $\mathrm{C}-7^{\prime}, \mathrm{C}-11^{\prime}, \mathrm{C}-15^{\prime}$ & $135.09,135.04,134.99$ & $135.87,135.79$ & ${ }^{\prime}-$ \\
\hline $7^{\prime}-\mathrm{CH}_{3}, 11^{\prime}-\mathrm{CH}_{3}, 15^{\prime}-\mathrm{CH}_{3}$ & $16.12,16.13,16.15$ & $16.10,16.13$ & ${ }^{\prime}+$ \\
\hline C-8', C-12', C-16' & $40.23,40.20$ & 40.81 & ${ }^{-}-$ \\
\hline C-9', C-13', C-17' & $27.23,27.12,27.11$ & $27.78,27.43,27.58$ & ${ }^{\prime}-$ \\
\hline C-18' & 125.04 & 125.91 & ${ }^{\prime}{ }^{\prime}$ \\
\hline C-19' & 131.07 & 131.99 & ${ }^{\prime}{ }^{\prime}$ \\
\hline $19^{\prime}-\mathrm{CH}_{3}$ & 17.73 & 17.75 & ${ }^{\prime}+$ \\
\hline $\mathrm{C}-20$ & 25.78 & 25.89 & ${ }^{\prime}+$ \\
\hline
\end{tabular}




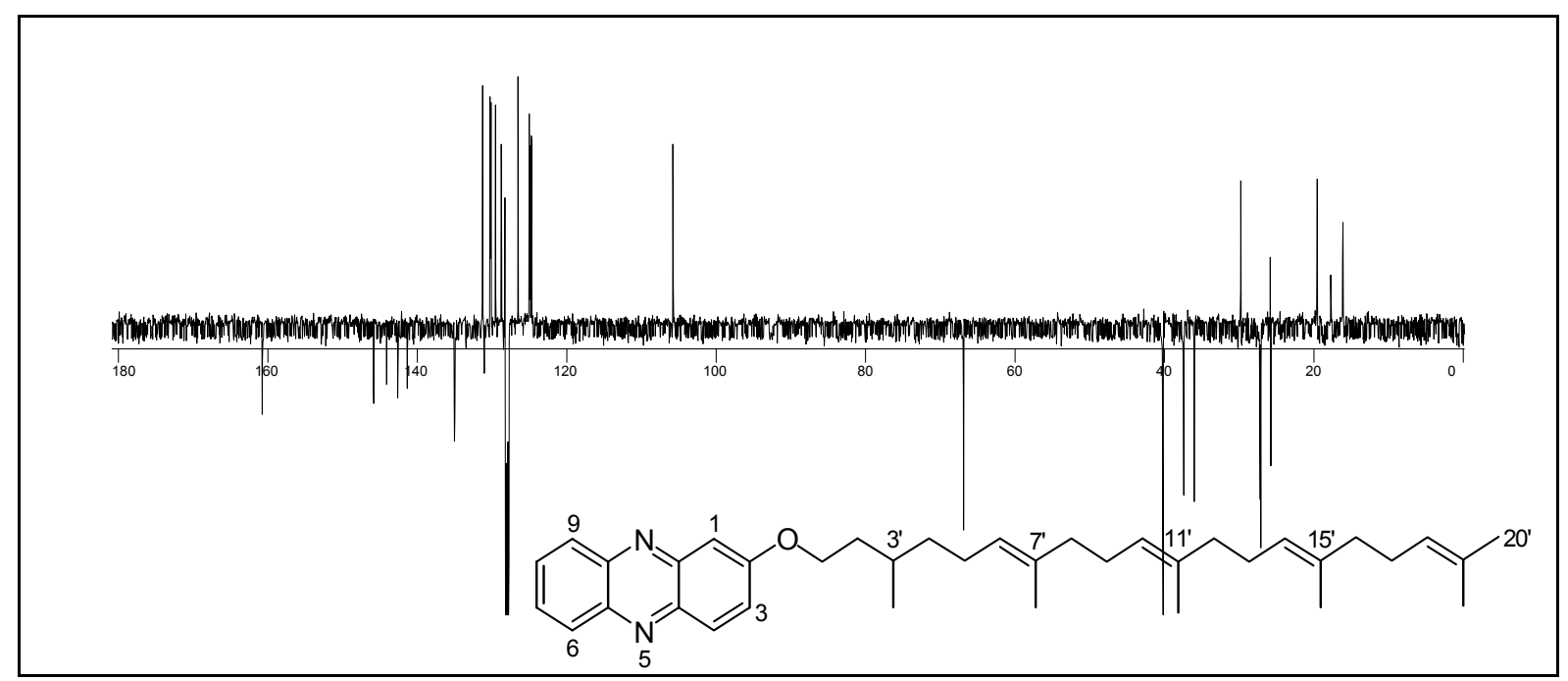

Abb. 26: ${ }^{13} \mathrm{C}$ APT NMR-Spektrum von natürlichem Methanophenazin (1) (125 MHz, $\mathrm{C}_{6} \mathrm{D}_{6}$ )

Von den 11 Signalen tertiärer Kohlenstoffatome werden vier Resonanzen bei $\delta=124.73$, 124.77, 124.93 und 125.04 ppm durch die olefinischen Atome C-6', C-10', C-14' und C-18' im Spektrum hervorgerufen. Die verbleibenden sieben Signale erscheinen im Aromatenbereich und werden dementsprechend zugeordnet. Eine auffallende Hochfeldverschiebung zu $\delta=105.80$ ppm erfährt das C-1 Atom. Hier wirkt sich der positive mesomere Effekt des an C2 gebundenen Sauerstoffatoms aus. Die Atome C-3 und C-4 mit Signalen bei $\delta=126.53$ und 131.29 ppm können mit Hilfe eines zweidimensionalen ${ }^{1} J(\mathrm{C}, \mathrm{H})$ Korrelationsspektrums (HMQC, Heteronuclear Multiple Quantum Correlation) eindeutig zugeordnet werden. Die chemischen Verschiebungen der verbleibenden vier tertiären Atome C-6, C-7, C-8 und C-9 werden den Signalen bei $\delta=130.26,130.17$ ppm (für C-6/C-9) und $\delta=129.56,128.78$ ppm (für C-7/C-8) zugeordnet. Eine eindeutige Bestimmung hätte die Korrelation zwischen einem zugeordneten Atom, beispielsweise C-1/1-H oder C-4/4-H, mit mindestens einem der vier unbestimmten C- bzw. H-Atome erfordert. Dies ist jedoch nicht möglich, denn die vier tertiären C-Atome bilden eine, durch die beiden Stickstoffatome isolierte Teilstruktur, die von den H,H COSY- und HMBC-Korrelationsmethoden (Heteronuclear Multiple Bond Correlation) nicht erreicht wird.

Die Resonanzen von 10 Methylengruppen werden durch die sekundären C-Atome in der Seitenkette hervorgerufen. Dabei besetzen sieben $\mathrm{CH}_{2}$-Gruppen die Allylpositionen der Doppelbindungen. Die Kohlenstoffatome C-8', C-12' und C-16' befinden sich am Kopfende des jeweiligen ungesättigten isoprenoiden Bausteins (Abbildung 27). Diese drei Atome zeigen zwei Signale bei $\delta=40.23$ und 40.20 ppm. Die Resonanzen bei $\delta=25.83,27.23,27.12$ und 
27.11 ppm werden C-5', C-9', C-13' und C-17' zugeordnet. Sie besetzen die Allylstellungen am Schwanzende der jeweiligen isoprenoiden Teilstrukturen. ${ }^{[37]}$

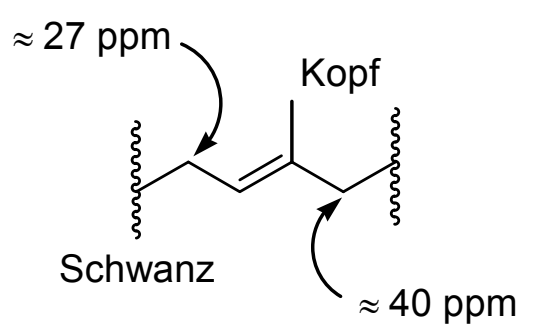

Abb. 27: ${ }^{13} \mathrm{C}$ NMR-Verschiebungen der Allylkohlenstoffatome einer Isopreneinheit

Durch die direkte Verbindung mit einem Sauerstoffatom findet sich das Signal für C-1' bei einer für Ether typischen Verschiebung von $\delta=66.91 \mathrm{ppm}$. Zwei weitere sekundäre Kohlenstoffatome (C-2' und C-4') resonieren bei $\delta=36.01$ und 37.45 ppm. Zusammen mit der Resonanz des tertiären C-Atoms C-3' bei $\delta=29.80$ ppm und der Resonanz des primären C-Atoms der Methylgruppe 3'- $\mathrm{CH}_{3}$ bei $\delta=19.56 \mathrm{ppm}$, läßt sich ein gesättigter isoprenoider $\mathrm{C}_{5} \mathrm{H}_{10}$-Baustein als weitere Teilstruktur erkennen. Die Zuordnung insbesondere der diastereotopen Protonen 2'- $\mathrm{H}_{\mathrm{A}}$, 2' $^{\prime}-\mathrm{H}_{\mathrm{B}}$ sowie 4'- $\mathrm{H}_{\mathrm{A}}$ und 4'- $\mathrm{H}_{\mathrm{B}} \mathrm{zu}$ den Atomen C-2' und C-4' wird durch die Auswertung eines HMQC-Spektrums ermöglicht. Die Verknüpfung der Kohlenstoffatome C-1', C-2' $\quad$ C-3', 3 ' $-\mathrm{CH}_{3}$ und $\mathrm{C}-4$ ' wird durch $\mathrm{H}, \mathrm{H}-$ Korrelationsspektroskopie der zugehörigen H-Atome sichergestellt (Abbildung 28).

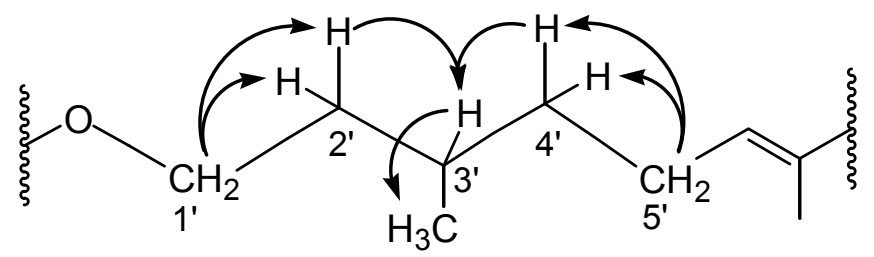

Abb. 28: H,H-Korrelationen im gesättigten Teil der Seitenkette von 1

Neben der oben genannten Methylgruppe an C-3 findet man noch drei weitere Resonanzen dieser Art bei $\delta=16.12,16.13$ und 16.15 ppm. Sie werden den 7' $-\mathrm{CH}_{3}, 11^{\prime}-\mathrm{CH}_{3}$ und $15^{\prime}-\mathrm{CH}_{3}$ zugeordnet. Die Signale bei $\delta=17.73$ und 25.78 ppm charakterisieren die Kohlenstoffatome $19^{\prime}-\mathrm{CH}_{3}$ und C-20' und markieren das Ende einer ungesättigten Terpeneinheit. Dabei resoniert die (E)-ständige Methylgruppe (C-20') im Vergleich mit der (Z)-ständigen (19'$\mathrm{CH}_{3}$ ) bei tieferem Feld. Durch Analyse des HMQC-Spektrums ordnet man ihnen die im ${ }^{1} \mathrm{H}$ 
NMR-Spektrum bei $\delta=1.58(Z)$ und $1.69 \mathrm{ppm}(E)$ in Erscheinung tretenden Resonanzen zu. Die H,H COSY- und die HMBC- Daten von 1 sind in Tabelle 5 zusammengefaßt.

Die Verknüpfung der nun vorliegenden Teilstrukturen, einer gesättigten und vier ungesättigten Isopreneinheiten untereinander sowie ihre Verbindung mit dem Heteroaromaten, wurde durch die Auswertung eines zweidimensionalen ${ }^{3} J(\mathrm{C}, \mathrm{H})$ Korrelationsspektrums (HMBC) ermittelt. Die Frage, ob die $\mathrm{C}_{5} \mathrm{H}_{8}$-Strukturteile in KopfSchwanz-, Schwanz-Schwanz- oder Kopf-Kopf-Manier untereinander verknüpft sind, war von besonderem Interesse.

Tabelle 5: H,H COSY- und HMBC-Daten von Methanophenazin (1) (500 MHz, $\mathrm{C}_{6} \mathrm{D}_{6}$ )

\begin{tabular}{|c|c|c|c|}
\hline Position & $\delta_{H}[p p m]$ & H,H COSY & НМВС \\
\hline 1 & 7.53 & $3-\mathrm{H}$ & $\mathrm{C}-2,4 \mathrm{a}, 10 \mathrm{a}$ \\
\hline 3 & 7.32 & $1-\mathrm{H}, 4-\mathrm{H}$ & $\mathrm{C}-1,4,4 \mathrm{a}$ \\
\hline 4 & 8.12 & $3-\mathrm{H}$ & $\mathrm{C}-2,4 \mathrm{a}$ \\
\hline 6 & 8.31 & $7-\mathrm{H}, 8-\mathrm{H}$ & C-5a, 7, 8, 9a, \\
\hline 7 & 7.29 oder 7.35 & 6-H, 8-H, 9-H & C-5a, $6,8,9$ \\
\hline 8 & 7.35 oder 7.29 & 6-H, 7-H, 9-H & C-6, 7, 9, 9a \\
\hline 9 & 8.31 & 8-H, 7-H & C-4a, 5a, 7, 8 \\
\hline $1 '-A$ & 3.79 & $1^{\prime}-\mathrm{H}_{\mathrm{B}}, 2^{\prime}-\mathrm{H}_{\mathrm{A}, \mathrm{B}}$ & $\mathrm{C}-2,2^{\prime}, 3^{\prime}$ \\
\hline $1 '-B$ & 3.77 & $1^{\prime}-\mathrm{H}_{\mathrm{A}}, 2^{\prime}-\mathrm{H}_{\mathrm{A}, \mathrm{B}}$ & $\mathrm{C}-2,2^{\prime}, 3^{\prime}$ \\
\hline $2^{\prime}-A$ & 1.44 & $2-\mathrm{H}_{\mathrm{B}}, 1^{\prime}-\mathrm{H}_{\mathrm{A}, \mathrm{B}}$ & $\mathrm{C}-1^{\prime}, 3^{\prime}, 4^{\prime}, 3^{\prime}-\mathrm{CH}_{3}$ \\
\hline 2'-B & 1.72 & $2-\mathrm{H}_{\mathrm{A}}, 1^{\prime}-\mathrm{H}_{\mathrm{A}, \mathrm{B}}, 3^{\prime}-\mathrm{H}$ & $\mathrm{C}-1^{\prime}, 3^{\prime}, 4^{\prime}, 3^{\prime}-\mathrm{CH}_{3}$ \\
\hline 3 & $1.60-1.65$ & $2-\mathrm{H}_{\mathrm{A}, \mathrm{B}}, 3^{\prime}-\mathrm{CH}_{3}, 4^{\prime}-\mathrm{H}_{\mathrm{B}}$ & $\mathrm{C}-2^{\prime}, 4^{\prime}$ \\
\hline $3^{\prime}-\mathrm{CH}_{3}$ & 0.86 & $3^{\prime}-\mathrm{H}$ & $\mathrm{C}-3^{\prime}, 4^{\prime}$ \\
\hline $4^{\prime}-\mathrm{A}$ & $1.18-1.27$ & $3^{\prime}-\mathrm{H}_{\mathrm{B}}, 5-\mathrm{H}_{2}$ & $\mathrm{C}-3^{\prime}, 5^{\prime}, 3^{\prime}-\mathrm{CH}_{3}$ \\
\hline $4^{\prime}-\mathrm{B}$ & $1.32-1.39$ & $3^{\prime}-\mathrm{H}, 4^{\prime}-\mathrm{H}_{\mathrm{B}}, 5-\mathrm{H}_{2}$ & $\mathrm{C}-3^{\prime}, 5^{\prime}, 3^{\prime}-\mathrm{CH}_{3}$ \\
\hline 5 & $2.00-2.30$ & $4^{\prime}-\mathrm{H}_{\mathrm{A}, \mathrm{B}}, 6^{\prime}-\mathrm{H}$ & $\mathrm{C}-3^{\prime}, 4^{\prime}, 6^{\prime}, 7^{\prime}$ \\
\hline 6 & & $5^{\prime}-\mathrm{H}_{2}, 7^{\prime}-\mathrm{CH}_{3}, 8-\mathrm{H}_{2}$ & $\mathrm{C}-5^{\prime}, 8^{\prime}, 7^{\prime}-\mathrm{CH}_{3}$ \\
\hline 10 & $5.27-5.38$ & $9^{\prime}-\mathrm{H}_{2}, 11^{\prime}-\mathrm{CH}_{3}, 12-\mathrm{H}_{2}$ & $\mathrm{C}-9 ', 12^{\prime}, 11^{\prime}-\mathrm{CH}_{3}$ \\
\hline $14^{\prime}$ & & $13^{\prime}-\mathrm{H}_{2}, 15^{\prime}-\mathrm{H}_{2}, 16-\mathrm{H}_{2}$ & $\mathrm{C}-13^{\prime}, 16^{\prime}, 15^{\prime}-\mathrm{CH}_{3}$, \\
\hline
\end{tabular}


Tabelle 5: Fortsetzung

\begin{tabular}{|c|c|c|c|}
\hline Position & $\delta_{H}[p p m]$ & H,H COSY & НМВС \\
\hline $7^{\prime}-\mathrm{CH}_{3}$ & & 6'-H & C-6', 7', 8' \\
\hline $11^{\prime}-\mathrm{CH}_{3}$ & $1.63,1.64,1.65$ & $10^{\prime}-\mathrm{H}$ & C-10', 11', 12' \\
\hline $15^{\prime}-\mathrm{CH}_{3}$ & & $15^{\prime}-\mathrm{H}$ & C-14', 15', 16' \\
\hline 8', & & $6^{\prime}-\mathrm{H}, 9^{\prime}-\mathrm{H}$ & $\mathrm{C}-6^{\prime}, 7^{\prime}, 9^{\prime}, 7^{\prime}-\mathrm{CH}_{3}$ \\
\hline $12^{\prime}$, & $2.00-2.30$ & $10^{\prime} \mathrm{H}, 13^{\prime}-\mathrm{H}$ & $\mathrm{C}-10^{\prime}, 11^{\prime}, 13^{\prime}, 11^{\prime}-\mathrm{CH}_{3}$ \\
\hline $16^{\prime}$ & & $14^{\prime}-\mathrm{H}, 17^{\prime}-\mathrm{H}$ & $\mathrm{C}-14^{\prime}, 15^{\prime}, 17^{\prime}, 15^{\prime}-\mathrm{CH}_{3}$ \\
\hline 9' & & $8^{\prime}-\mathrm{H}_{2}, 10^{\prime}-\mathrm{H}$ & C-8', 10', 11' \\
\hline $13^{\prime}$ & $2.00-2.30$ & $12^{\prime}-\mathrm{H}_{2}, 14^{\prime}-\mathrm{H}_{2}$ & $\mathrm{C}-12^{\prime}, 14^{\prime}, 15^{\prime}$ \\
\hline $17^{\prime}$ & & $16^{\prime}-\mathrm{H}_{2}, 18^{\prime}-\mathrm{H}_{2}$ & $\mathrm{C}-16^{\prime}, 18^{\prime}, 19^{\prime}$ \\
\hline $18^{\prime}$ & 5.25 & $17^{\prime}-\mathrm{H}_{2}, 19^{\prime}-\mathrm{H}_{2}, 20^{\prime}-\mathrm{H}_{3}$ & $19^{\prime}-\mathrm{CH}_{3}, \mathrm{C}-20^{\prime}$ \\
\hline $19^{\prime}-\mathrm{CH}_{3}$ & 1.58 & $18^{\prime}-\mathrm{H}$ & $\mathrm{C}-19^{\prime}, 18^{\prime}$ \\
\hline 20 & 1.69 & $18^{\prime}-\mathrm{H}$ & $\mathrm{C}-19^{\prime}, 18^{\prime}, 19^{\prime}-\mathrm{CH}_{3}$ \\
\hline
\end{tabular}

An zentraler Stelle der Untersuchung des HMBC-Spektrums stehen die Signale der 1'- $\mathrm{H}_{2}$ Protonen. Sie lassen sich mit den Kohlenstoffatomen C-2 und C-3' korrelieren und bestätigen die Annahme einer Etherbrücke als Bindeglied zwischen dem Heteroaromaten und der Seitenkette. Zusätzlich wird klar, daß der aromatische Kern mit dem Schwanzende der gesättigten $\mathrm{C}_{5} \mathrm{H}_{10}$-Teilstruktur verknüpft ist (Abbildung 30).

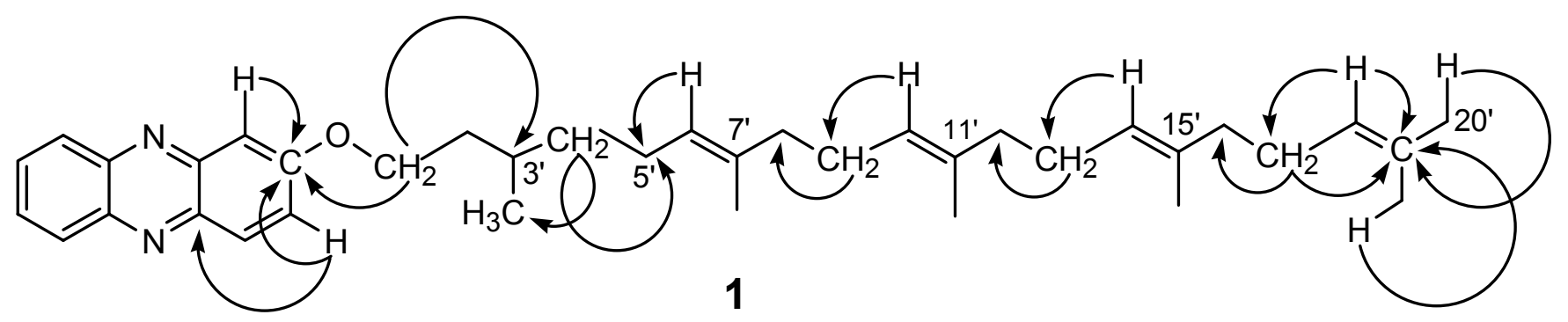

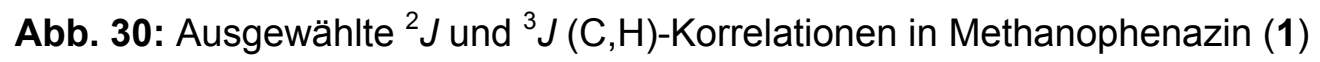

Eine weitere wichtige Korrelation besteht zwischen C-5' und den Protonen 4'- $\mathrm{H}_{2}$ und 6'- $\mathrm{H}$, denn sie belegt die Verbindung des Kopfs der gesättigten mit dem Schwanz der ersten ungesättigten Isopreneinheit. Dieses Kopf-Schwanz-Verknüpfungsmuster gilt auch für den Rest der Seitenkette, wie die Wechselwirkungen zwischen 9'- $\mathrm{H}_{2}$ und C-8', 13'- $\mathrm{H}_{2}$ und C-12' und 
17'- $\mathrm{H}_{2}$ sowie C-16' zeigen. Am Ende der Seitenkette befinden sich die beiden Methylgruppen $19^{\prime}-\mathrm{CH}_{3}$ und $20^{\prime}-\mathrm{H}_{3}$. Deren Protonen korrelieren mit dem charakteristisch zu $\delta=131.07 \mathrm{ppm}$ verschobenen quartären Kohlenstoffatom C-19', welches seinerseits zusätzlich mit den 17'$\mathrm{H}_{2}$ Protonen in Kontakt steht. Die Wechselwirkung des vinylischen Protons 18'-H mit dem CAtom 19 '- $\mathrm{CH}_{3}$ bestimmt dessen Position am Ende der terpenoiden Kette.

Ein Vergleich der Daten ${ }^{13} \mathrm{C}$ NMR-chemischer Verschiebungen von Dolicholen - das sind polyisoprenoide Alkohole mit unterschiedlicher Anzahl nicht konjugierter, Kopf-Schwanz verknüpfter $(E)$ - und $(Z)$-konfigurierter Doppelbindungen - mit den eigenen experimentell ermittelten Werten zeigt, daß die chemischen Verschiebungen der Methylengruppen des Kopfes der Isopreneinheiten am stärksten von der Konfiguration der Doppelbindung beeinflußt werden. ${ }^{[38]}$ Ist diese Methylengruppe Teil einer $(E)$-Doppelbindung, so resoniert sie bei $\delta \approx 40 \mathrm{ppm}$. Im Falle einer $(Z)$-Doppelbindung erwartet man das Signal bei $\delta \approx 32 \mathrm{ppm}$ (Abbildung 31).
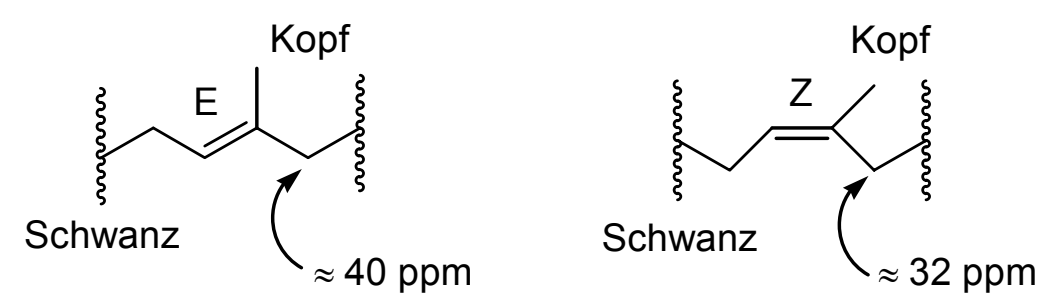

Abb. 31: Chemische Verschiebungen der Methylengruppen am Kopfende von (E)- und (Z)konfigurierten Isopreneinheiten ${ }^{[38]}$

Im Experiment wurden für die chemischen Verschiebungen der entsprechenden Atome C-8', C-12' und C-16' Werte von $\delta=40.23$ und 40.20 ppm gefunden. Daraus folgt, daß Methanophenazin (1) über eine all-(E)-konfigurierte isoprenoide Seitenkette verfügt.

\subsection{Zusammenfassung}

Der durch Extraktion von Cytoplasmamembranen des Archaeabakteriums Methanosarcina mazei Gö1 isolierte Naturstoff Methanophenazin (1) wurde chromatographisch unter Anwendung von HPLC-Techniken gereinigt. Seine Summenformel wurde durch hochauflösende Massenspektrometrie bestimmt. Die Struktur des neuen Naturstoffs wurde durch ein- und zweidimensionale Kernresonanzexperimente (HMQC, H,H COSY, HMBC) ermittelt. 
Methanophenazin (1) ist ein Sesterterpenether des 2-Hydroxyphenazins (2). Die Seitenkette besteht aus einem gesättigten und vier ungesättigten isoprenoiden Bausteinen, die regelmäßig in Kopf-Schwanz-Manier miteinander verknüpft und einheitlich $(E)$-konfiguriert sind. Am Schwanzende der gesättigten $\mathrm{C}_{5} \mathrm{H}_{10}$-Einheit befindet sich die Etherfunktionalität, die den terpenoiden Rest mit C-2 des Phenazins verknüpft. Der in kleinen Mengen gewonnene empfindliche Naturstoff ist das erste aus Archaea isolierte Phenazinderivat. 


\section{Vorkommen und Biosynthese von Phenazinen}

\subsection{Vorkommen von Phenazinen ${ }^{[39]}$}

Während Methanophenazin (1) das erste Phenazinderivat ist, das aus Archaea isoliert wurde, sind Phenazine aus Bakterien schon seit längerem bekannt. Dazu zählen die extracellulären Pigmente Pyocyanin (14), Iodinin (15) und Chlororaphin (16), die an ihren Farben erkannt und aus Pseudomonas-Stämmen bzw. aus Brevibakterium iodinum isoliert wurden. ${ }^{[40,41,42]}$<smiles>C[n+]1c2ccccc2nc2c([O-])cccc21</smiles>

14<smiles>O=[N+]([O-])c1c2cccc(O)c2[n+]([O-])c2cccc(O)c12</smiles>

15<smiles>NC(=O)c1cccc2c1Nc1ccccc1N2</smiles>

Man hat bis heute etwa 75 weitere natürlich vorkommende Phenazin-Derivate gefunden, die ausschließlich von Eubakterien produziert werden. ${ }^{[43]}$ Ihnen allen gemeinsam ist der aromatische Phenazin-Grundkörper (Abbildung 32). Sie unterscheiden sich in der Art der Substituenten und im Substitutionsmuster voneinander.<smiles>[Y]c1ccc2nc3ccccc3nc2c1</smiles>

Phenazin

Abb. 32: Struktur von Phenazin

Hierbei werden bevorzugt die Positionen 1, 2, 4, 6 und 9 durch Carboxyl- und Hydroxylgruppen substituiert. Bekannt sind aber auch 2, 3, 7-trisubstituierte Verbindungen. Meistens 
werden nur zwei bis vier, selten sechs oder mehr Substituenten am Ring vorgefunden. Ein häufig auftretendes und vielfältig variiertes Motiv ist das des 1,6-disubstituierten Phenazingrundgerüsts. In Abbildung 33 sind einige Beispiele natürlich vorkommender Phenazine dargestellt.<smiles>O=C(O)c1c(O)ccc2nc3ccccc3nc12</smiles>

2-Hydroxyphenazin1-carbonsäure<smiles>O=C(O)c1c(O)c(O)c(O)c2nc3ccccc3nc12</smiles>

\section{2,3,4 -Trihydroxyphenazin-} 1-carbonsäure<smiles>O=C(O)c1cccc2nc3c(O)cccc3nc12</smiles>

6-Hydroxyphenazin1-carbonsäure<smiles>O=C(O)c1c(O)ccc2nc3c(C(=O)O)c(O)c(O)cc3nc12</smiles>

2,3,7-Trihydroxyphenazin1,6-dicarbonsäure

Abb. 33: Strukturen einiger natürlich vorkommender Phenazin-Derivate

Aus den Gattungen Pseudomonas, Streptomyces, Brevibakterium, Microbispora und Sporangium sind eine Vielzahl phenazinproduzierender Mikroorganismen bekannt. Mehr als die Hälfte der bekannten Phenazine werden von Pseudomonaden produziert. So wurden in den zurückliegenden Jahrzehnten allein aus dem Stamm Pseudomonas aureofaciens 14 Verbindungen wie beispielsweise Phenazin-1-carbonsäure (17), 2-Hydroxyphenazin (2), 2,3-Dihydroxyphenazin (18), Phenazin-1,6-dicarbonsäure (19), u. a. isoliert. ${ }^{[4]}$ 
<smiles>O=C(O)c1cccc2nc3ccccc3nc12</smiles>

17<smiles>Oc1cc2nc3ccccc3nc2cc1O</smiles>

18<smiles>Oc1ccc2nc3ccccc3nc2c1</smiles>

2<smiles>O=C(O)c1cccc2nc3c(C(=O)O)cccc3nc12</smiles>

19

An zweiter Stelle in Bezug auf die Produktion von Phenazinderivaten rangiert nach den Pseudomonaden die Gattung Streptomyces. Die aus Kulturen verschiedener Streptomyceten isolierten Metabolite sind teilweise identisch mit denen aus Pseudomonaden, teilweise unterscheiden sie sich nur geringfügig am Substitutionsmuster. Da Streptomyceten als Quelle vieler physiologisch aktiver Substanzen bekannt sind, wurden die meisten der aus ihnen gewonnenen Verbindungen auf ihre biologische Aktivität überprüft. Viele der oben genannten Phenazine zeigen antibiotische Wirkung. Modelluntersuchungen an Pyocyanin (14), Iodinin (15), Phenazin-1,6-dicarbonsäure (19) u. a. schreiben die antibiotische Aktivität der planaren aromatischen Phenazinstruktur, ihrer Fähigkeit zur Einlagerung in die DNA und der damit einhergehenden Inhibierung der DNA-abhängigen RNA-Synthese zu. ${ }^{[45]}$ Besonders erwähnenswert sind die Verbindungen 1,2,6-Trimethoxyphenazin (Mycomethoxin A) und 6Methoxyphenazin-1-carbonsäuremethylester (Mycomethoxin B), die aus Streptomyces luteoreticuli isoliert wurden (Abbildung 34). ${ }^{[46]}$ Sie wirken gegen pathogene Mycobakterien, einschließlich der Stämme, die sich als resistent gegenüber den gebräuchlichen Wirkstoffen Streptomycin und Isoniazid zeigen. Auf der Suche nach einem Antibiotikum gegen Mycobakterium tuberculosis fand man in Streptomyces misakiensis einen Produzenten der tuberkulostatisch wirkenden Phenazin-1-carbonsäure (17) (Tubermycin B). Die aus einer Sorangium-Spezies isolierte Verbindung Myxin wird als Breitspektrum-Antibiotikum eingesetzt. ${ }^{[47]}$ 
<smiles>COc1ccc2nc3c(OC)cccc3nc2c1OC</smiles>

Mycomethoxin A<smiles>COC(=O)c1cccc2nc3c(OC)cccc3nc12</smiles>

Mycomethoxin B<smiles>COc1cccc2c1[n+]([O-])c1cccc(O)c1[n+]2[O-]</smiles>

Myxin

Abb. 34: Strukturen von Mycomethoxin A, Mycomethoxin B und Myxin

Im Unterschied zu den Pseudomonaden sind Streptomyceten in der Lage, neben den oben erwähnten einfachen Phenazinen auch komplexere Phenazin-Derivate mit ungewöhnlichen Seitenketten aufzubauen. Als Beispiele mögen die aus Streptomyces endus sub. sp. aureus isolierten Substanzen Sendomycin A und Sendomycin B dienen (Abbildung 35). Sie zeigen neben einer breiten antibiotischen Aktivität auch karzinostatische Wirkung. ${ }^{[48]}$<smiles>Cc1ccc(C)c2nc3c(C(=O)NC4=C(O)CCC4O)cccc3nc12</smiles>

Sendomycin A

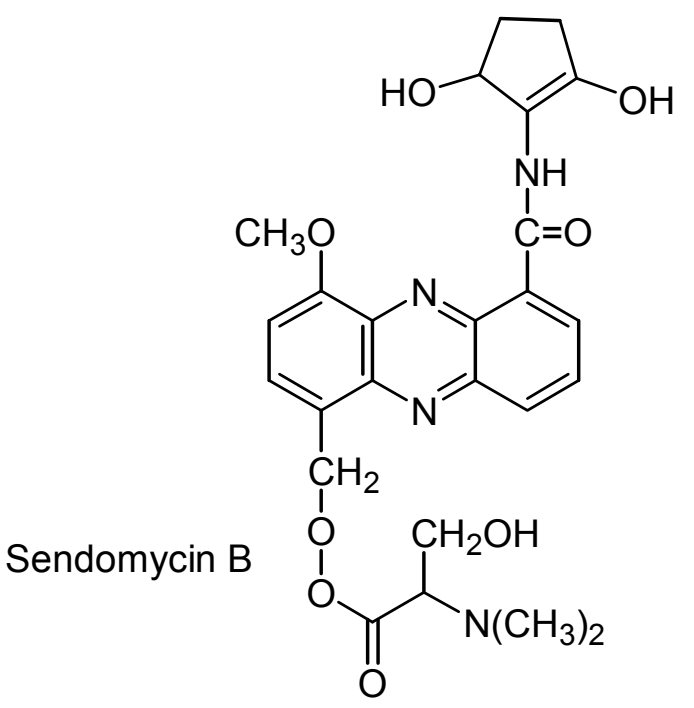

Abb. 35: Strukturen von Sendomycin A und Sendomycin B

Entdeckungen der jüngeren Zeit sind die gegen Bakterien und Tumoren wirksamen dimeren Phenazinderivate Esmeraldin A, Esmeraldin B sowie Saphenamycin, welche aus Streptomyces antibioticus Tü 2706 isoliert wurden (Abbildung 36). ${ }^{[49]}$ 
<smiles>[R2]OC(=O)c1cc2c(c3nc4c(C(C)O[R])cccc4nc13)C(C)c1cccc3c1N2c1cccc(C([R])O[R])c1N3</smiles>

Esmeraldin A $\mathrm{R}=\mathrm{H}, \mathrm{R}^{\prime}=$ verschiedene Fettsäuren Esmeraldin $\mathrm{B}$ $R=H, R^{\prime}=2-H y d r o x y-6-m e t h y l b e n z o y l$<smiles>[R]OC([R])c1cccc2nc3c(C(C)O[R])cccc3nc12</smiles>

Saphenamycin $\mathrm{R}=\mathrm{H}$ $\mathrm{R}^{\prime}=$ 2-Hydroxy-6-methylbenzoyl

Abb. 36: Strukturen von Esmeraldin A, Esmeraldin B und Saphenamycin

\subsection{Biosynthese von Phenazinen}

Die Biogenese phenazinhaltiger Verbindungen in Archaea ist noch ungeklärt. Modellvorstellungen hierzu orientieren sich an der Biosynthese von Phenazinen in Eubakterien, auf deren Aufklärung einige Mühe verwandt worden ist. ${ }^{[50]}$

Die Entstehung von Phenazinen verläuft zunächst nach dem bekannten Shikimisäure-Weg der Biosynthese von aromatischen Aminosäuren (Abbildung 37). Der erste Schritt dieser Sequenz ist eine intermolekulare Aldolreaktion zwischen Phosphoenolpyruvat (A) und D-Erythrose-4phosphat (B) zu 3-Desoxy-D-arabinoseheptulosonsäure-7-phosphat (C). Daran anschließend erfolgt der Ringschluß zu Dehydrochininsäure (D). Dies geschieht nach der Eliminierung des Phosphatrestes in $\mathbf{C}$ durch eine intramolekulare Aldolreaktion. Die Eliminierung von Wasser aus D führt zu 5-Dehydroshikimisäure (E). Die nachfolgende Reduktion ihrer Carbonylgruppe in 3-Position mit Hilfe von NADPH $+\mathrm{H}^{+}$ergibt Shikimisäure (F). Sie kondensiert nach Phosphorylierung der 3-OH-Funktionalität durch ATP mit einem Molekül Phosphoenolpyruvat (A) zum 3-Enolpyruvyl-Zwischenprodukt (H), welches abschließend durch eine Eliminierung von Phosphorsäure in Chorisminsäure (I) transformiert wird. Sie ist die gemeinsame Vorstufe aller drei aromatischen Aminosäuren Tryptophan (L), Phenylalanin (M) und Tyrosin (N). 


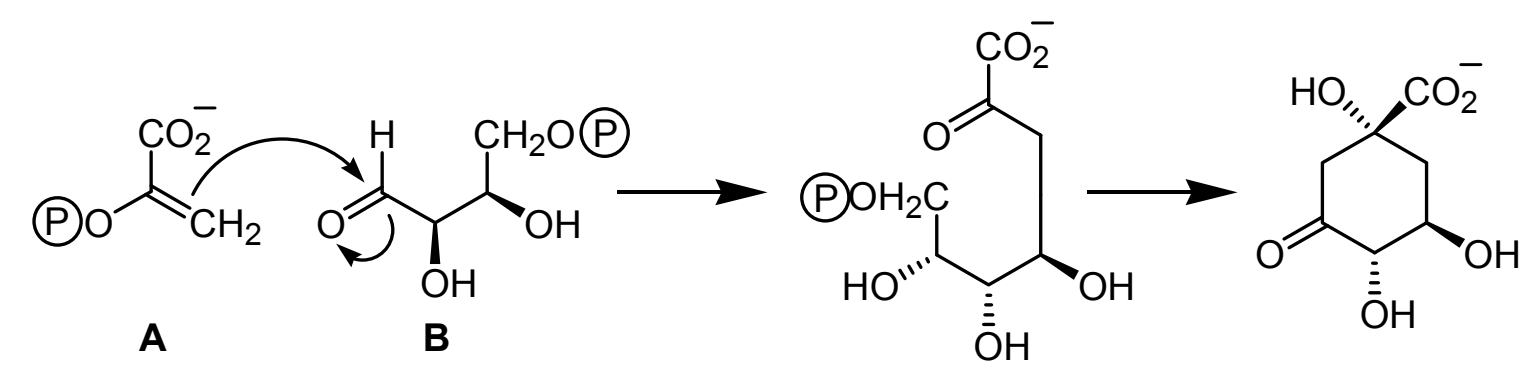

A

B

C

D

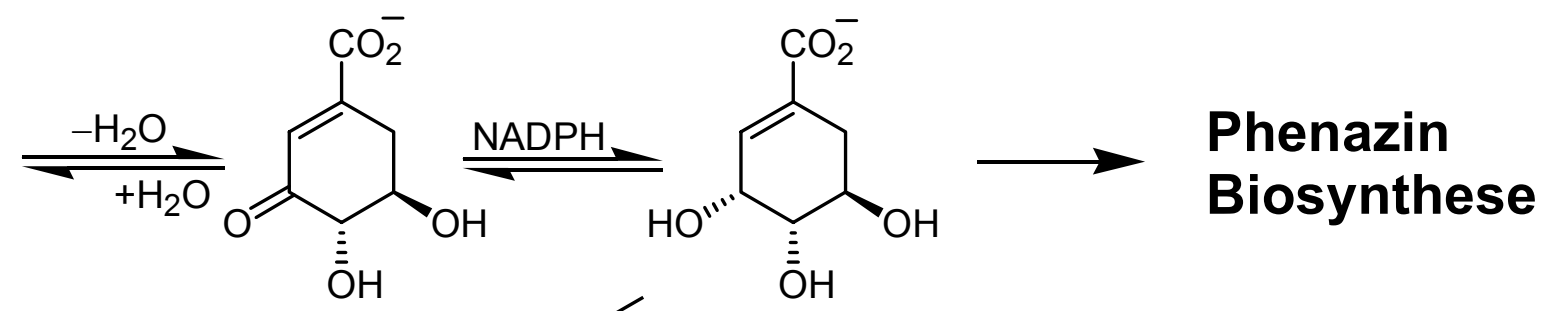

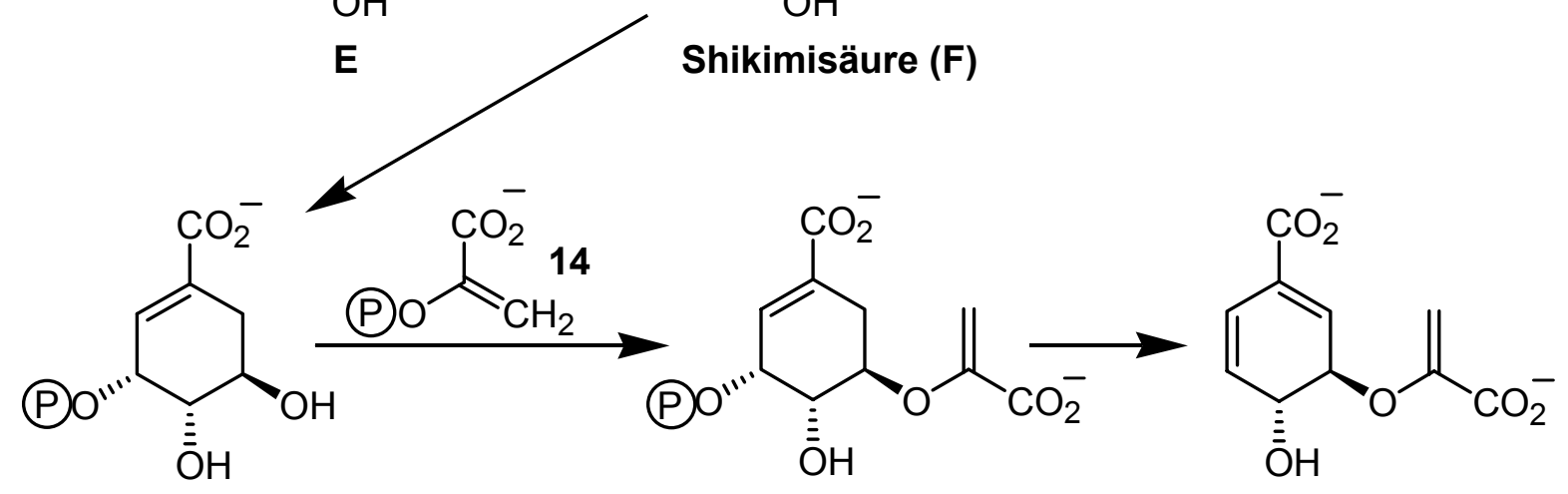

G

H Chorisminsäure (I)<smiles>Nc1ccccc1C(=O)O</smiles>

Anthranilsäure (J)<smiles>CCCCCCC(N)Cc1c[nH]c2ccccc12</smiles>

Tryptophan (L)
Phenylalanin (M)<smiles>CCCCCCCCCCC(=O)OCC(=O)OC</smiles><smiles>N[C](Cc1ccccc1)C(=O)O</smiles>

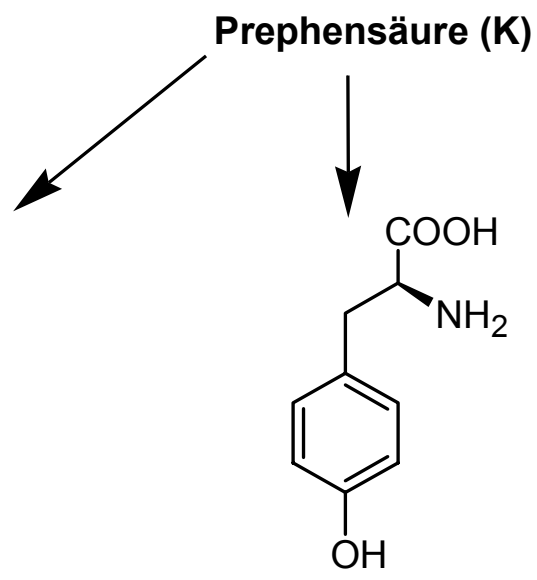

Tyrosin (N)

Abb. 37: Schema zum Shikimisäure-Weg der Biosynthese aromatischer Aminosäuren 
Die Aminierung von Chorisminsäure (I) führt über Anthranilsäure (J) zu Tryptophan (L). Dagegen verläuft die Bildung von Phenylalanin (M) und Tyrosin (N) über Prephensäure (K) als Zwischenverbindung, die nach Art einer Claisen-Umlagerung aus Chorisminsäure (I) entsteht. Der in die aromatischen Aminosäuren eingebaute Stickstoff entstammt der Aminosäure L-Glutamin, die in vielen Biosynthesereaktionen als Aminogruppendonator dient (Abbildung 38).<smiles>NC(=O)CC[C@H](N)C(=O)O</smiles>

L-Glutamin

Abb. 38: Struktur von L-Glutamin

Alle in Abbildung 37 dargestellten Umwandlungen, wie etwa die Aldolreaktionen, die Reduktion, die Eliminierungen und die Umlagerung, verlaufen stereospezifisch. Die Mechanismen sind gut untersucht und der stereochemische Verlauf dieser Sequenz ist durch zahlreiche Experimente belegt. ${ }^{[51]}$

Der auf den ersten Blick plausible Weg zu Phenazinen durch Kondensation von zwei Molekülen Anthranilsäure (J) fand bislang keine experimentelle Bestätigung. Ausführliche Fütterungsexperimente mit ${ }^{14} \mathrm{C}$ markierter Anthranilsäure $(\mathbf{J})$ und ${ }^{14} \mathrm{C} /{ }^{3} \mathrm{H}$ markierter Shikimisäure (F) bei der Biosynthese von Pyocyanin (14) durch Pseudomonas aeroginosa bzw. bei der Biosynthese von Iodinin (15) durch Brevibakterium iodinum zeigten, daß zwei Moleküle Shikimisäure (F) als biogenetische Vorstufe zu deren Aufbau dienen. ${ }^{[52]}$

Diese Abzweigung vom Shikimisäure-Weg zum Phenazin-Biosyntheseweg, ohne Beteiligung von Anthranilsäure (J), wurde durch Versuche mit Mutanten von Pseudomonas aeruginosa bestätigt. Sie produzierten Pyocyanin (14), obwohl sie weder in der Lage waren, Shikimisäure (F) abzubauen, noch die Fähigkeit zur Synthese von Aminosäuren besaßen. ${ }^{[53]}$

Grundlegende Untersuchungen der Stickstoffquelle wurden von R. B. Herbert und A. Römer und seinen Mitarbeitern an Brevibakterium iodinumn und Pseudomonas aureofacinens unternommen. ${ }^{[54]}$ Sie fanden durch Einbauversuche mit ${ }^{15} \mathrm{~N}$ markiertem L-Glutamin heraus, daß der Amid-Stickstoff von L-Glutamin als Stickstoffquelle beim biologischen Aufbau von Phenazinen dient. Die Suche nach einer intermediär aus Shikimisäure (F) durch Transaminierung gebildeten Zwischenverbindung, die dann in einen aromatischen PhenazinTricyclus übergeführt wird, blieb bislang ohne Erfolg. 
Als allgemeiner Vorläufer der Biogenese von Phenazinen wird Phenazin-1,6-dicarbonsäure (19) angenommen, die als Primärprodukt aus Shikimisäure (20) aufgebaut wird. Häufig vorkommende Reaktionen am aromatischen Ring sind Hydroxylierungen. Zur Einführung des Sauerstoffs wird die entsprechende Stelle des Aromaten zunächst epoxidiert und das Epoxid dann selektiv geöffnet. Dieser Schritt wird oft von einer Decarboxylierung begleitet. Die Biogenese von 2-Hydroxyphenazin (2) in Pseudomonas aureofaciens folgt vermutlich diesem Prinzip. Am Beginn der Synthese steht Phenazin-1,6-dicarbonsäure (19). Durch Decarboxylierung entsteht Phenazin-1-carbonsäure (17), die zur Einführung der Hydroxylgruppe in 2-Stellung zunächst in das entsprechende Epoxid 21 übergeführt wird. Die Epoxidöffnung verläuft unter Verlust der Carboxylgruppe als Kohlendioxid und bildet 2-Hydroxyphenazin (2). ${ }^{[5]}$<smiles>O=C([O-])C1=C[C@@H](O)[C@H](O)[C@H](O)C1</smiles>

20

19

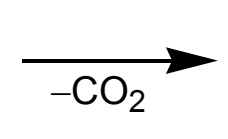<smiles>O=C(O)c1cccc2nc3ccccc3nc12</smiles>

17

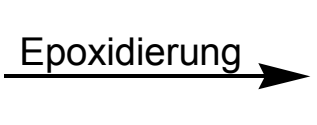<smiles>Oc1ccc2nc3ccccc3nc2c1</smiles>

2

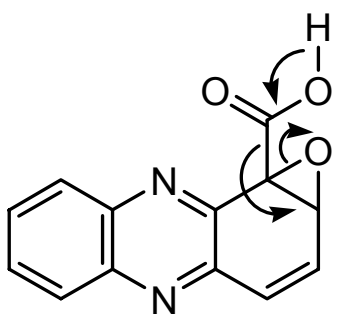

21

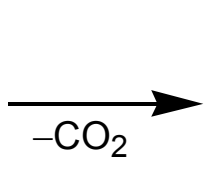

Die meisten Studien hierzu wurden an Brevibacterium iodinum und an verschiedenen Pseudomonas-Stämmen durchgeführt. Wie Untersuchungen gezeigt haben, lassen sich die hierbei gefundenen Prinzipien auch auf Stämme der Gattung Streptomyces übertragen. ${ }^{[56]}$ 
Die Entdeckung der ungewöhnlichen Phenazine Esmeraldin A und B in Streptomyces antibioticus Tü 2706 wirkte stimulierend auf Untersuchungen zur Biogenese dieser Strukturen. ${ }^{[43]}$ Die Bedeutung der Phenazinderivate für ihre Produzenten ist ungeklärt. Es ist bezeichnend für eine Vielzahl von Mikroorganismen unterschiedlicher Gattungen, daß die von ihnen produzierten Sekundärmetabolite keine offensichtliche physiologische Funktion übernehmen. ${ }^{[57]}$ Das Ausscheiden von antibiotisch wirksamen Sekundärmetaboliten interpretiert man daher als Maßnahme zu Schaffung eines Standortvorteils für den Produzenten, und zwar durch Inhibieren oder Abtöten von Nahrungskonkurrenten. ${ }^{[58]}$

Obwohl manche Phenazine an Redoxprozessen beteiligt sind, gab es vor dem Auffinden von Methanophenazin (1) keinen Anhaltspunkt für eine physiologische Funktion eines PhenazinDerivates in einer Atmungskette. ${ }^{[59]}$ 


\section{Synthese von 2-Hydroxyphenazin (2)}

\subsection{Wege zu Phenazinen ${ }^{[60]}$}

Bei den meisten Synthesen von Phenazinderivaten ist der Aufbau des zentralen heterocyclischen Rings der kritische Schritt der Synthesesequenz. Weil der desaktivierte PhenazinGrundkörper elektrophile aromatische Substitutionsreaktionen nur schwer eingeht, werden Derivate oft durch die Fusion entsprechend substituierter Vorläufer synthetisiert.

Einen direkten Zugang zu Phenazinen ermöglicht z. B. die intermolekulare Kondensation von $o$-Phenylendiamin (5) mit $o$-Benzochinonen bzw. 1,2-Naphtochinonen. ${ }^{[61]}$ Die benötigten $o$ Chinone 22 werden dabei in situ aus den entsprechenden Catecholen durch Oxidation mit Cer(IV)sulfat oder Mangandioxid hergestellt und dann mit $o$-Phenylendiamin (5) abgefangen.<smiles>Nc1ccccc1N</smiles>

5<smiles>[R]C1=CC(=O)C(=O)C=C1</smiles>

22<smiles>[R]c1cccc2nc3ccccc3nc12</smiles>

23

Nach diesem Syntheseprinzip, jedoch unter Umgehung der Probleme, die aus der Herstellung der $o$-Chinone resultieren, synthetisierte Ris Phenazin (26) durch Erhitzen einer Mischung aus 5 und Catechol (24) im verschlossenem Rohr bei $220-240{ }^{\circ} \mathrm{C} .{ }^{[62]}$ Das als Primärprodukt entstehende 5,10-Dihydrophenazin (25) wird an der Luft zu Phenazin (26) oxidiert.<smiles>Nc1ccccc1O</smiles>

5

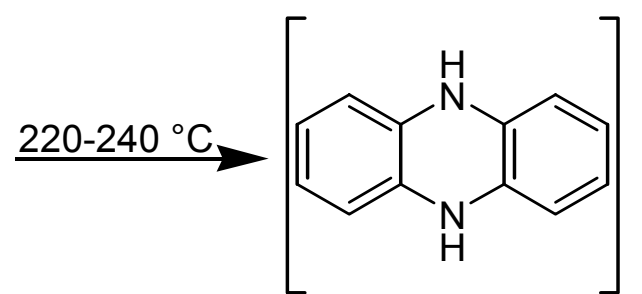

25<smiles></smiles>

26

Ein alternativer Weg zum Aufbau der Phenazinstruktur ist die intramolekulare Cyclisierung von ortho-substituierten Diphenylaminderivaten unter reduktiven oder oxidativen Bedingungen. Das in die angelsächsische Literatur auch als Vivian-Methode ${ }^{[63]}$ eingegangene Verfahren wurde bereits viel früher von Nietzki und Ernst ${ }^{[64]}$ zur Phenazin-Synthese durch Cyclisierung von 2-Aminodiphenylamin (30) eingesetzt. Die durch ipso-Substitution des reaktiven 
Chloratoms in $o$-Nitrochlorbenzol (27) durch Anilin (28) und anschließender Reduktion der Nitrogruppe erhaltene Verbindung 30 cyclisiert in Gegenwart von Braunstein und Eisenspänen zu Phenazin (26).<smiles>O=[N+]([O-])c1ccccc1Cl</smiles>

27<smiles>Nc1ccccc1</smiles>

28

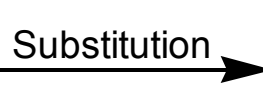<smiles>O=[N+]([O-])c1ccccc1Nc1ccccc1</smiles>

29<smiles>Nc1ccccc1Nc1ccc(F)cc1</smiles>

30

Vivian, Hartwell und Waterman ${ }^{[65]}$ nutzten diese Methode zur Darstellung von 2-Chlorphenazin (33), das sie dann in 2-Hydroxyphenazin (2) umwandeln konnten. Die Substitution des Chloratoms in $\mathbf{3 3}$ durch eine Hydroxylgruppe gelingt allerdings erst nach Umwandlung der beiden zentralen Stickstoffatome in die entsprechenden Oxide. Die $N$-Oxide können abschließend mit alkalischer Natriumhydrogensulfitlösung desoxygeniert werden.<smiles>Nc1ccccc1</smiles>

31

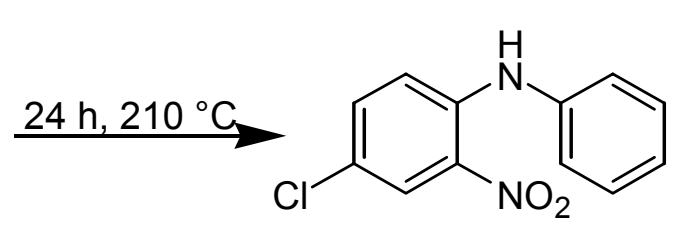

32
$\mathrm{FeC}_{2} \mathrm{O}_{4} \cdot 2 \mathrm{H}_{2} \mathrm{O}, \mathrm{Pb}$, $240{ }^{\circ} \mathrm{C}, 16 \mathrm{~h}$<smiles>Cc1ccc2nc3ccccc3nc2c1</smiles>

33
$\mathrm{HOAc}, 30 \% \mathrm{H}_{2} \mathrm{O}_{2}$, $40 \mathrm{~h}, 55^{\circ} \mathrm{C}$<smiles>[O-][n+]1c2ccccc2[n+]([O-])c2cc(Cl)ccc21</smiles>

34<smiles>[O-][n+]1c2ccccc2[n+]([O-])c2cc(O)ccc21</smiles>

35
1. $\mathrm{NaHSO}_{3}, \mathrm{NaOH}$ 2. $\mathrm{HOAc}, \mathrm{H}_{2} \mathrm{O}$<smiles>Oc1ccc2nc3ccccc3nc2c1</smiles> 
Eine weitere, interessante Methode zur Synthese zahlreicher Phenazinderivate ist die BeirutReaktion. ${ }^{[66]}$ Bei dieser Reaktion kondensiert man Benzofuroxan (36) mit einer Reihe unterschiedlicher Substrate unter Bildung der korrespondierenden Phenazin- $N$-oxide. So reagiert 36 bereitwillig mit 1,2-Cyclohexandion (37) in Gegenwart sekundärer und tertiärer Amine zu einer Mischung aus 1-Hydroxyphenazinmono-(38) und di-N-oxid (39). Nach Zugabe einer Persäure zur Reaktionsmischung erhält man ausschließlich 1-Hydroxyphenazin5,10-dioxid (38) als Produkt.<smiles>O=C1C=CC=CC1=O</smiles>

36

37

1. $\mathrm{HNEt}_{2}$ 2. $\mathrm{HOAc}, \mathrm{H}_{2} \mathrm{O}$<smiles></smiles>

38

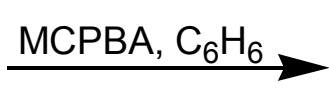<smiles>[O-][n+]1c2ccccc2[n+]([O-])c2c(O)cccc21</smiles>

39

Bemerkenswert ist die Reaktion von Benzofuroxan (36) mit den Phenolat-Anionen von Phenol, Resorcin und Hydrochinon, aber auch mit Benzochinon, weil hierbei 2-Hydroxyphenazin-5,10-dioxid (42) in allen Fällen als einziges Produkt gebildet wird. Es kann dann mit Natriumdithionit zu 2-Hydroxyphenazin (2) reduziert werden. ${ }^{[65]}$ Ley und seine Mitarbeiter $^{[67]}$ nutzten Hydrochinon (41) zur Gewinnung von 42.<smiles>[O-][n+]1onc2ccccc21</smiles>

36
41
$\stackrel{\mathrm{NaOH}, \mathrm{H}_{2} \mathrm{O}}{\longrightarrow}$<smiles>[O-][n+]1c2ccccc2[n+]([O-])c2cc(O)ccc21</smiles>

42

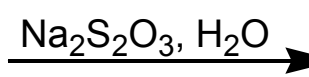<smiles>Oc1ccc2nc3ccccc3nc2c1</smiles> 
Die in der Regel bei dieser Reaktion in guten Ausbeuten erhaltenen $N$-Oxide können mit einer Reihe von Reduktionsmitteln, wie beispielweise Natriumdithionit, Phosphorylchlorid oder $\mathrm{Pd} / \mathrm{C}$-Wasserstoff zu den entsprechenden Phenazinen desoxygeniert werden.

Das zur Synthese von 1 benötigte 2-Hydroxyphenazin (2) wurde auf direktem Wege nach der Vorschrift von Kehrmann und Cherpillod ${ }^{[68]}$ bzw. der Vorschrift von $O t t^{[69]}$ hergestellt. Diese Methoden versprachen gute Ausbeuten und gingen von einfachen, im Handel günstig erhältlichen Substanzen aus.

\subsection{Synthese von 2-Hydroxyphenazin (2) nach Kehrmann und Cherpillod ${ }^{[68]}$}

Ausgangspunkt dieser Synthese ist p-Benzochinon (43), das mit Essigsäureanhydrid im Sauren zu 1,2,4-Triacetoxybenzol (44) umgesetzt wird. ${ }^{[70]}$ Eine anschließende saure Verseifung der drei Estergruppen mit konzentrierter Salzsäure in Methanol ergibt 1,2,4Trihydroxybenzol (45) in guter Ausbeute. ${ }^{[70]}$ Das graue Rohprodukt läßt sich durch Sublimation im Vakuum bei $10^{-2}$ torr reinigen. Man erhält so 45 als weiße analysenreine Substanz.<smiles>CC(=O)Oc1ccc(OC(C)=O)c(OC(C)=O)c1</smiles>

Das zur Kondensation mit $o$-Phenylendiamin (5) erforderliche 2-Hydroxybenzochinon (46), wird nach einer Vorschrift von Willstätter und Müller ${ }^{[71]}$ aus 1,2,4-Trihydroxybenzol (45) durch Oxidation mit Silber(I)oxid gewonnen. Das empfindliche gelbe Chinon 46, das an Luft zur Zersetzung neigt, wird am besten ohne weitere Aufreinigung mit $o$-Phenylendiamin (5) zu Reaktion gebracht. Nach Kehrmann und Cherpillod ${ }^{[68]}$ suspendiert man das Chinon 46 in konzentrierter Essigsäure und gibt $o$-Phenylendiamin (5) innerhalb weniger Minuten hinzu. Im weiteren Verlauf scheiden sich aus der zunächst blutroten und danach gelb gefärbten 
Lösung Kristalle von 2-Hydroxyphenazin (2) ab, die durch Zugabe von „reichlich“ Wasser vervollständigt wird. Nach Aufarbeitung und Reinigung beträgt die Ausbeute an $\mathbf{2}$ laut Literatur $87 \%$. $^{[68]}$<smiles>Oc1ccc(O)c(O)c1</smiles>

45

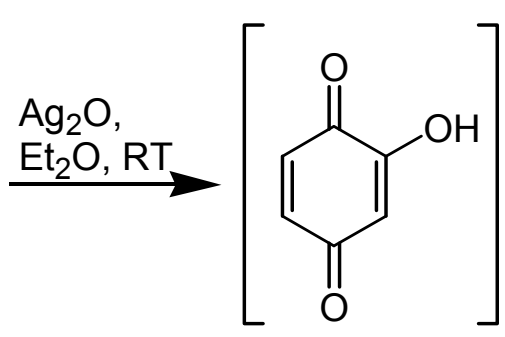

46

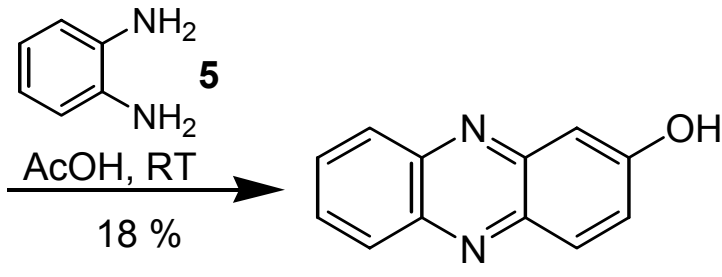

2

Bei eigenen Versuchen konnte nach Zugabe von 5 zur essigsauren Chinon-Suspension ebenfalls eine dunkle Rotfärbung der Reaktionsmischung beobachtet werden. Hierauf trat, im Gegensatz zu den Angaben in der Literatur, keine Gelbfärbung, sondern eine Schwarzfärbung des Ansatzes ein. Auf dem Boden des Glaskolbens ließ sich bei Betrachtung im Gegenlicht schemenhaft ein schwarzer Niederschlag erkennen, auf den die Zugabe von Wasser wenig Einfluß hatte. Filtration, Reinigung durch Umfällen, Entfernen des Kristallwassers bei $150{ }^{\circ} \mathrm{C}$ und Chromatographie an $\mathrm{SiO}_{2}$ ergaben das Produkt mit Ausbeuten zwischen 10 und $18 \%$. Untersuchungen des Filtrats durch dünnschichtchromatographische Analyse zeigten neben einem schwarzen Startfleck noch überschüssiges $o$-Phenylendiamin (5) und sehr wenig weiteres 2-Hydroxyphenazin (2), das aber nicht isoliert wurde.

Kehrmann und Cherpillod ${ }^{[68]}$ führen die anfänglich auftretende intensive rote Färbung der Reaktionsmischung auf ein offenes Anilid als Zwischenprodukt zurück, das dann durch Ringschluß zum Phenazin abreagiert. Um eine Verbesserung der Ausbeuten zu erzielen, wurden neben Variationen der Stöchiometrie der Reaktionspartner auch die Reaktionsführung geändert. So versuchte man beispielsweise durch Erhitzen der dunkelroten Suspension aus $\mathbf{4 6}$ und 5 die Phenazinbildung zu verbessern. Dies gelang jedoch nicht.

Die unbefriedigenden Ausbeuten bei dieser Reaktion gaben Anlaß zur Suche nach einer alternativen Synthesemethode. 


\subsection{Synthese von 2-Hydroxyphenazin (2) nach $\mathrm{Ott}^{[69]}$}

Bei seinen Untersuchungen über Chinone fand $R$. Ott, daß die Kondensation von $p$-Benzochinon (43) mit $o$-Phenylendiamin (5) in alkoholischer Lösung direkt zu 2-Hydroxyphenazin (2) führt. Diese Methode besticht nicht nur durch die Verwendung leicht zugänglicher Substrate und der Einfachheit in der Durchführung, sondern auch durch ihre hohe Ausbeute, die von $\mathrm{Ott}^{[69]}$ mit ,etwa $90 \%$ “ angegeben wurde.<smiles>O=C1C=CC(=O)C=C1</smiles>

43<smiles>Nc1ccccc1N</smiles>

5

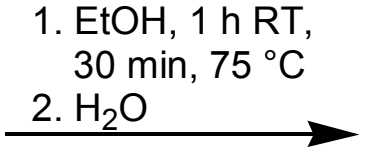

$10 \%$<smiles>Oc1ccc2nc3ccccc3nc2c1</smiles>

2

Eigene Versuche unter Anwendung der Ott'schen Versuchsvorschrift ergaben bei der Umsetzung von 43 mit 5 zwar das angestrebte Produkt 2, doch blieben die Ausbeuten mit 6 bzw. $10 \%$ deutlich hinter der Erwartung zurück. Angesichts der mageren Ergebnisse wurden keine weiteren Anstrengungen zur Optimierung dieses Verfahrens unternommen.

Wie die genannten Versuche zeigen, ist der Zugang zu 2-Hydroxphenazin (2) auf direktem Wege, also durch intermolekulare Kondensation von 2-Hydroxybenzochinon (46) bzw. oBenzochinon (43) mit $o$-Phenylendiamin (5) möglich. Hierbei gute Ausbeuten zu erzielen ist hingegen problematisch. Die Anwendung einer mehrstufigen Synthese, beispielweise der Beirut-Reaktion, ${ }^{[66]}$ erscheint unter diesen Bedingungen erfolgversprechender, wurde aber nicht durchgeführt. 


\subsection{Diskussion der spektroskopischen Daten von 2-Hydroxyphenazin (2)}<smiles>Oc1ccc2nc3ccccc3nc2c1</smiles>

Reines 2-Hydroxyphenazin (2) ist ein gelber Feststoff, der im UV-Spektrum bei $\lambda=216,253$, 355 und $391 \mathrm{~nm}$ Absorptionsmaxima aufweist. Sein IR-Spektrum verrät die Hydroxylfunktion durch eine breite Bande im Bereich von $\widetilde{v}=3420-2595 \mathrm{~cm}^{-1}$. Die C-HStreckschwingungen werden durch die ausgeprägten Absorptionen der Wasserstoffbrückenbindungen der OH-Gruppe überlagert. Zahlreiche Absorptionen, beispielsweise bei $\widetilde{v}=1632,1602,1476$ und $1452 \mathrm{~cm}^{-1}$, werden durch Streckschwingungen der aromatischen $\mathrm{C}=\mathrm{C}$-Doppelbindungen hervorgerufen.

Im ${ }^{1} \mathrm{H}$ NMR-Spektrum (Abbildung 39) erkennt man das breite mit $\mathrm{D}_{2} \mathrm{O}$ austauschbare Signal des sauren Hydroxylprotons bei $\delta=10.85 \mathrm{ppm}$. Alle anderen Signale liegen im aromatischen Bereich und sind durch die Gegenwart der elektronegativen Stickstoffatome zu tiefem Feld verschoben. Am stärksten wirkt sich das auf die Protonen in Position 4, 6 und 9 aus, die mit Verschiebungen zu $\delta=8.10,8.11$ und 8.15 ppm bei tiefstem Feld resonieren. Die Hydroxylgruppe hat den stärksten Einfluß auf das Proton in 1-Position. Es erfährt einen charakteristischen Hochfeldshift und resoniert bei $\delta=7.34 \mathrm{ppm}$. Sein Signal wird durch eine ${ }^{4} J$ Kopplung mit dem Proton an C-4 zum Dublett aufgespalten. 


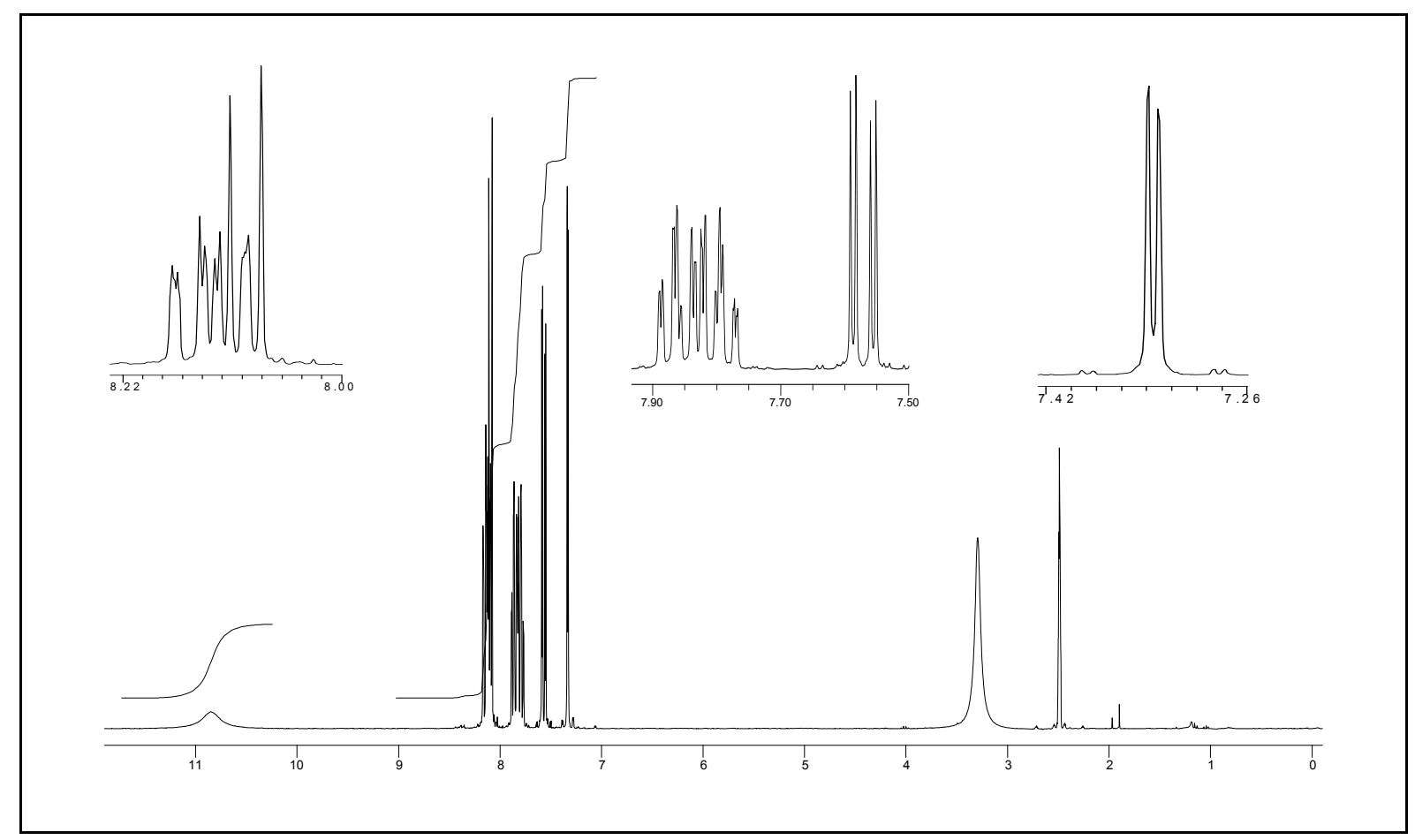

Abb. 39: ${ }^{1} \mathrm{H}$ NMR-Spektrum von 2-Hydroxyphenazin (2) (300 MHz, DMSO[D $\left.\left.\mathrm{D}_{6}\right]\right)$

Die Analyse des ${ }^{13} \mathrm{C}$ NMR APT-Spektrums (Abbildung 40) zeigt erwartungsgemäß fünf quartäre und sieben tertiäre Kohlenstoffatome. Bemerkenswert hierbei sind die Verschiebungen der Atome C-1 und C-2. Analog den Verhältnissen im Protonen-Spektrum, erfährt das Kohlenstoffatom C-1 einen Hochfeldshift durch den positiven mesomeren Effekt des indirekt benachbarten Sauerstoffatoms. Sein Signal erscheint bei $\delta=107.07 \mathrm{ppm}$. Das Atom C-2 als Träger der Hydroxylgruppe wird durch dessen negativen induktiven Effekt am stärksten zu tiefem Feld verschoben. Es resoniert bei $\delta=159.60 \mathrm{ppm}$.

Das Signal des Kohlenstoffatoms C-3 erscheint bei $\delta=126.39$ ppm und unterscheidet sich damit kaum von den anderen tertiären Kohlenstoffatomen C-4, C-6, C-7, C-8 und C-9, die alle im Bereich zwischen $\delta=128.48$ und $130.70 \mathrm{ppm}$ resonieren. Für die vier quartären Kohlen-stoffatome C-4a, C-5a, C-9a und C-10a finden sich Signale im Bereich von $\delta=$ 139.47 bis $144.75 \mathrm{ppm}$. 


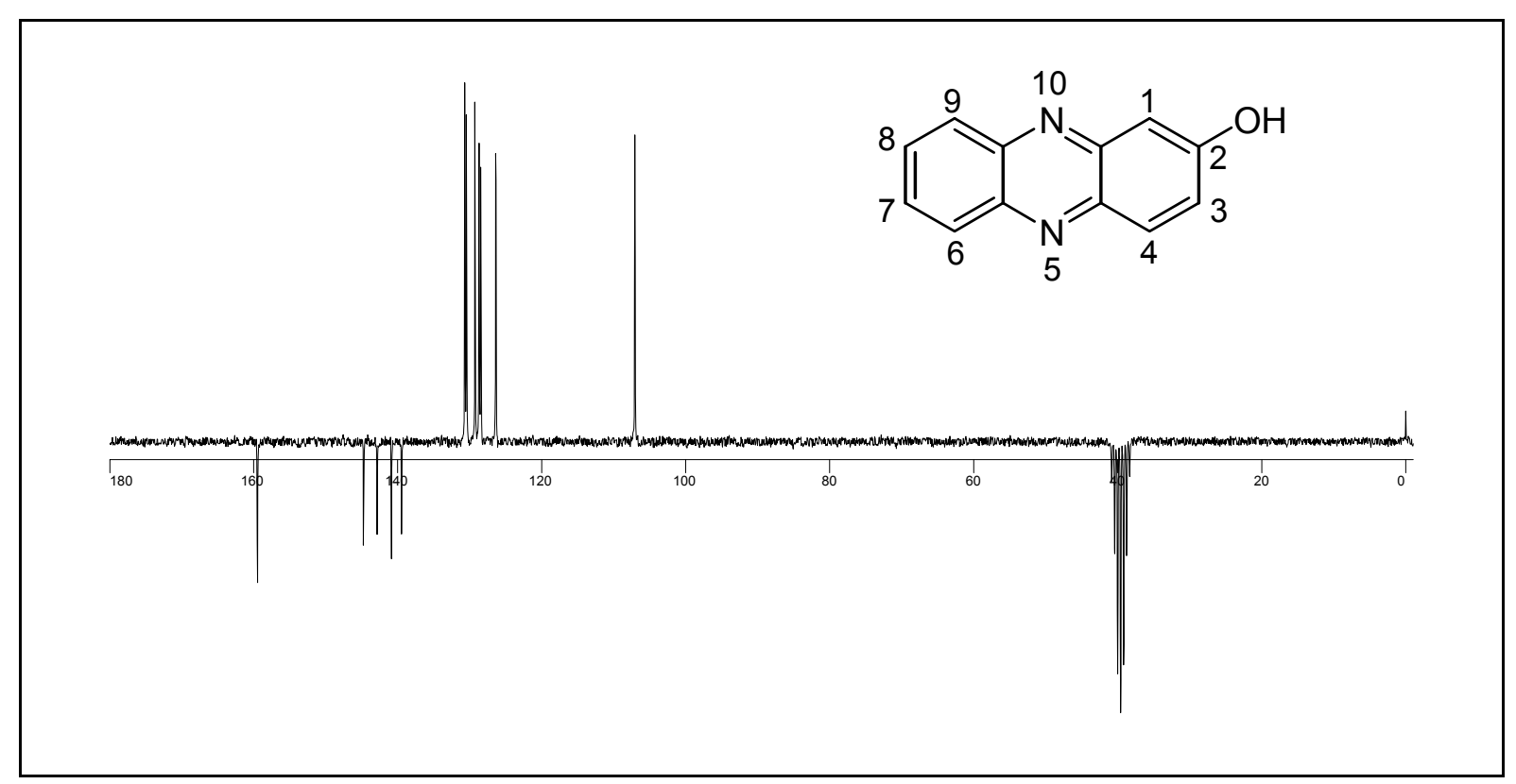

Abb. 40: ${ }^{13} \mathrm{C}$ NMR APT-Spektrum von 2-Hydroxyphenazin (2) $\left(50 \mathrm{MHz}, \mathrm{DMSO}\left[\mathrm{D}_{6}\right]\right)$

Im Massenspektrum erscheint das Molekülion mit $m / z=196$ als intensivstes Signal. Weitere charakteristische Signale zeigen die Fragmentionen $\left[\mathrm{M}^{+}-2 \mathrm{~N}\right]$ bei $m / z=168$ und $[168-\mathrm{CO}]$ bei $m / z=140$. Sie deuten auf die Extrusion der beiden Stickstoffatome und den anschließenden Verlust einer CO-Gruppe hin. ${ }^{[72]}$ 


\section{$7 \quad$ Synthese des Phenazinethers rac-51}

\subsection{Optimierung der Versuchsbedingungen}

Aus dem Retrosyntheseschema in Abbildung 15 ist zu ersehen, daß als letzte Stufe der Totalsynthese von Methanophenazin (1) eine Veretherung der isoprenoiden Seitenkette 3 mit 2Hydroxyphenazin (2) geplant ist. Um zu prüfen, ob sich dieser Schritt verwirklichen läßt, wurden vorab einige Versuche zur Ethersynthese zwischen 2-Hydroxyphenazin (2) und Citronellol (rac-47) als Modellverbindung durchgeführt. Das Ziel dieses Unterfangens war es, die optimalen Reaktionsbedingungen zur Durchführung der Transformation zu erarbeiten. Dabei galt es die beste Abgangsgruppe sowie die besten Reaktionbedingungen (Base, Lösungsmittel, Temperatur) herauszufinden. Die Überführung der Alkoholfunktion von rac47 in das ent-sprechende Mesylat rac-49 bzw. das Tosylat rac-48 wurde durch die Anwendung von Standardmethoden in guten Ausbeuten erreicht.<smiles>CC(C)=CCCC(C)CCO</smiles>

rac-47<smiles>CC(C)=CCCC(C)CCO</smiles>

rac-47

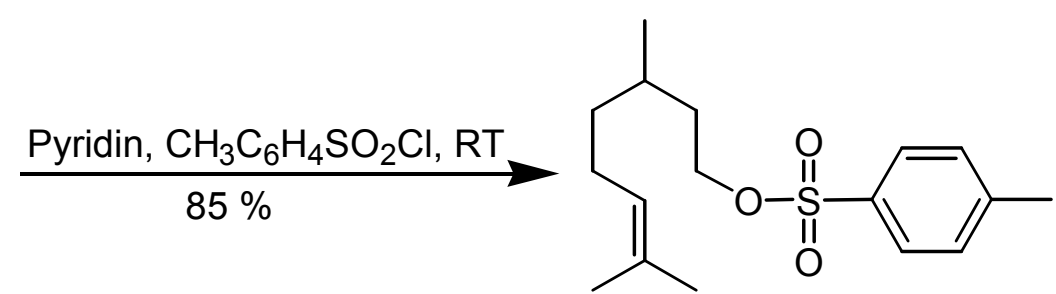

rac-48

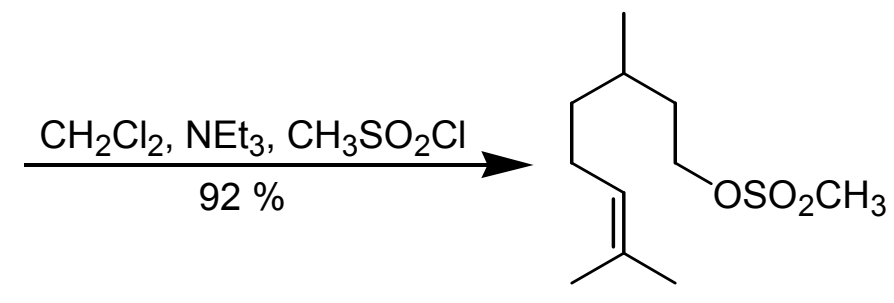

rac-49

Die Synthese des entsprechenden Bromids rac-50 gelang nach einer Vorschrift von Kocienski und seinen Mitarbeitern mit $86 \%$ Ausbeute. ${ }^{[73]}$ 


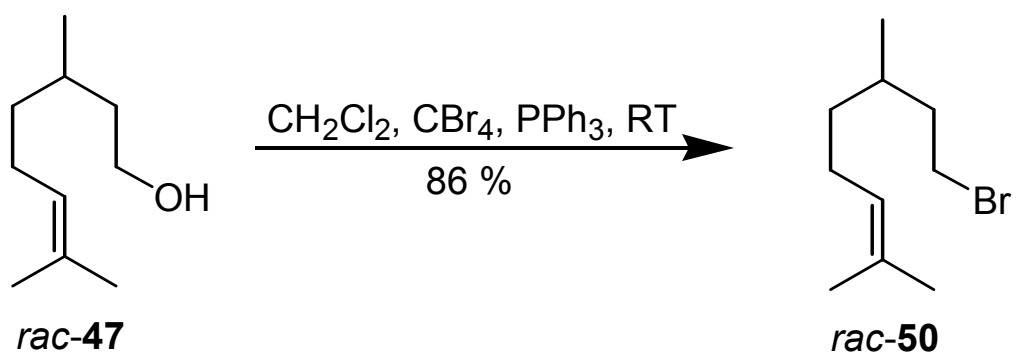

Erste Versuche mit Natriumhydrid als Base in Tetrahydrofuran ergaben bei langen Reaktionszeiten (>12 h) nur unvollständigen Umsatz und entsprechend niedrige Ausbeuten im Bereich von 40 bis $50 \%$. Hierbei war das Mesylat dem Tosylat als Abgangsgruppe gegenüber im Vor-teil. Die besten Ergebnisse erzielte man mit trockenem, feingemörsertem Kaliumcarbonat als Base in Dimethylformamid unter Einsatz des Mesylats rac-49 bei $100^{\circ} \mathrm{C}$. Versuche mit dem Bromid rac-50 ergaben keine besseren Ausbeuten an rac-51.

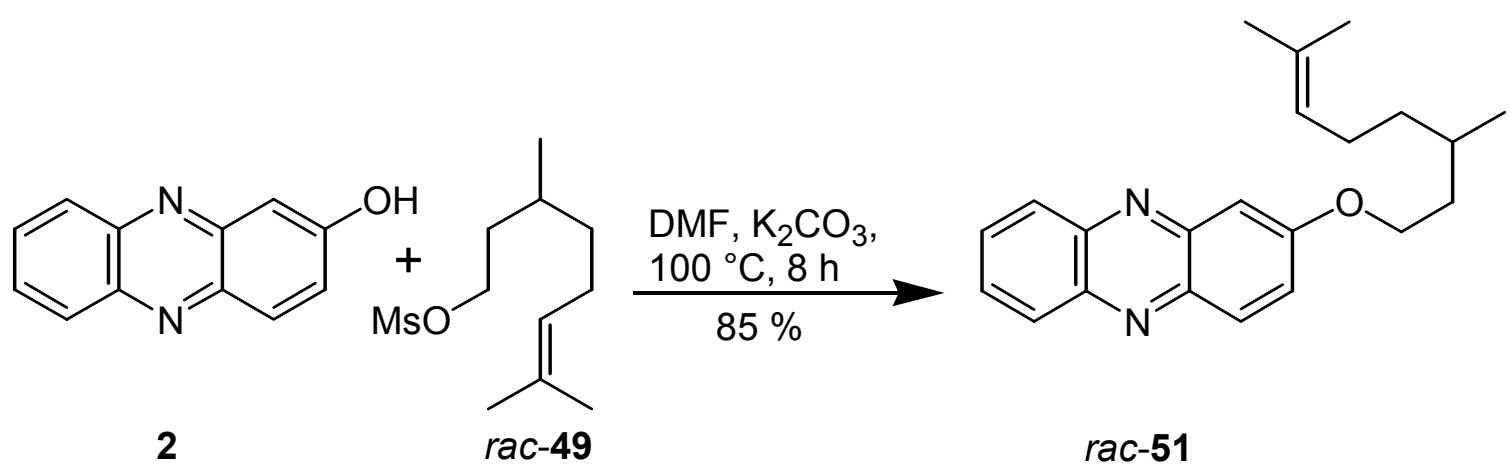

Eine Übertragung der Bedingungen zur Etherbildung dieser Modellexperimente auf die Natur-stoffsynthese verlief mit enttäuschendem Ergebnis (64\% Ausbeute), so daß eine andere Syn-thesemethode ausgearbeitet wurde. Eine Abwandlung der Vorschrift von Loupy und seinen Mitarbeitern ${ }^{[74]}$ brachte schließlich die angestrebte hohe Ausbeute. Mit gemörsertem Kalium-hydroxid als Base und unter Zusatz des Phasentransferkatalysators Aliquat 336 wurde rac-51 in einer Ausbeute von $96 \%$ isoliert. Während Loupy die Ethersynthese gänzlich ohne Solvens durchführte, bewährte sich beim eigenen Vorgehen Tetrahydrofuran als Lösungsmittel. 
<smiles>CCCCC(C)CCOc1ccc2nc3ccccc3nc2c1</smiles>

Vermutlich ist der Phasentransferkatalysator in der Lage, im heterogenen Reaktionsgemisch die Nucleophilie des Phenolat-Anions zu steigern, was sich günstig auf die Etherbildung auswirkt. Die besten Ergebnisse werden erzielt, wenn man Aliquat 336 über das katalytische Maß hinaus, in Mengen bis zu 30 Gewichtsprozent bezogen auf 2, einsetzt.

\subsection{Diskussion der spektroskopischen Daten von rac-51}<smiles>CC(Br)=CCCC(C)CCOc1ccc2nc3ccccc3nc2c1</smiles>

Der Phenazincitronellylether rac-51 ist ein gelbes Öl, das seine Absorptionsmaxima im UVSpektrum bei $\lambda=216,256,359$ und $391 \mathrm{~nm}$ hat. Im IR-Spektrum finden sich Signale bei $\widetilde{v}=$ 3060 und $3028 \mathrm{~cm}^{-1}$, die durch aromatische C-H-Streckschwingungen hervorgerufen werden. Die entsprechenden aliphatischen C-H-Streckschwingungen führen zu Absorptionsbanden bei $\widetilde{v}=2959$ bis $2855 \mathrm{~cm}^{-1}$. Ähnlich wie bei 2-Hydroxyphenazin (2) zeugen die Banden bei $\widetilde{v}=1632$ und $1605 \mathrm{~cm}^{-1}$ von den Streckschwingungen der $\mathrm{C}=\mathrm{C}$-Doppelbindungen des aromatischen Phenazingerüstes. Die Aryl-Alkylethergruppe zeigt die Absorption ihrer C-OStreckschwingung bei $\widetilde{v}=1281$ und $1197 \mathrm{~cm}^{-1}$.

Im ${ }^{1} \mathrm{H}$ NMR-Spektrum finden sich neben den Signalen der Protonen des Heteroaromaten, die bei der Interpretation des Spektrums von 2-Hydroxyphenazin (2) im vorangegangenen Kapitel ausführlich erörtert wurden, weitere Resonanzen, die Strukturelemente der ungesättigten Seitenkette anzeigen. Ein Resonanzsignal bei $\delta=1.00 \mathrm{ppm}$ zeugt von einer Methylgruppe, 
die mit einem tertiären Kohlenstoffatom direkt verbunden ist und durch eine ${ }^{3} J$-Kopplung mit dessen Proton charakteristisch zum Dublett aufspaltet. Die terminalen olefinischen Methylgruppen sind verantwortlich für zwei Singuletts bei $\delta=1.61$ uns $1.68 \mathrm{ppm}$. Die Resonanz des olefinischen Protons 6'-H erscheint bei $\delta=5.11 \mathrm{ppm}$. Die direkte Nähe des Sauerstoffatoms entschirmt die 1'- $\mathrm{H}_{2}$ Protonen und verursacht die Tieffeldverschiebung ihrer Signale zu $\delta=$ $4.21 \mathrm{ppm}$. Die chemische Verschiebung und die Muliplizität der aromatischen Protonen wird durch die Veretherung der Hydroxylgruppe nur wenig beeinflußt. Sie zeigen sich im Vergleich mit 2-Hydroxyphenazin (2) nur geringfügig verändert. Gleiches gilt auch für die Resonanzen der Kohlenstoffatome im ${ }^{13} \mathrm{C}$ NMR-Spektrum. Hier treten, zusätzlich zu den von 2 her bekannten Signalen der 12 aromatischen Kohlenstoffatome, noch zwei weitere Resonanzen bei $\delta=124.46$ und 131.32 ppm in Erscheinung. Zusammen mit den beiden Resonanzsignalen bei $\delta=17.65$ und 25.69 ppm markieren sie das Ende der ungesättigten Seitenkette und werden den Atomen C-6', C-7', 7'- $\mathrm{CH}_{3}$ und C-8' zugeordnet. Die für das direkt mit dem Sauerstoff verbundene Atom C-1' typische Resonanz findet sich bei $\delta=67.04$ ppm. 


\section{Synthese der $\mathrm{C}_{6}$-Bausteine rac-6 und (R)-6}

\subsection{Synthese von rac-6}

Der für die Synthese von rac-1 benötigte Baustein rac-6 wurde ausgehend von 3Methylpentan-1,5-diol (8) in wenigen Schritten hergestellt. Bei der Funktionalisierung dieses bifunktionellen Substrats 8, das über zwei äquivalente funktionelle Gruppen in gleicher chemischer Umgebung verfügt, gilt es zu beachten, daß der selektive Schutz von nur einer der beiden Alkoholfunktionen erschwert ist. Werden solche Substrate mit äquimolaren Mengen eines Silylierungsreagenzes unter Standardbedingungen zur Reaktion gebracht, resultieren häufig Gemische aus monofunktionalisiertem und bisfunktionalisiertem Produkt, sowie unverbrauchtem Substrat. Ist dieses Substrat gut verfügbar und leicht aus dem Reaktionsgemisch abzutrennen, setzt man es in großem Überschuß ein, um eine gleichzeitige Funktionalisierung beider reaktiven Positionen zu unterdrücken. Ist das Material jedoch wertvoll, dann ist es sinnvoll, eine Synthesemethode zur selektiven Monofunktionalisierung zu entwickeln. Von der Arbeitsgruppe um McDougal ${ }^{[75]}$ wurde eine solche Vorschrift zum selektiven Schutz symmetrischer Diole als tert.-Butyldimethylsilylether erarbeitet. Dabei setzt man äquimolare Mengen des entsprechenden Diols mit tert.-Butyldimethylsilylchlorid als Silylierungsreagenz um. Der Schlüssel für den Erfolg liegt in der Wahl der richtigen Base. Während Standardmethoden zum Silylieren von Alkoholen mit Triethylamin oder Pyridin als Base arbeiten, fanden McDougal und seine Mitarbeiter Natriumhydrid als geeignet, eine Monofunktionalisierung von bisfunktionellen Diolen zu erzielen. Im Falle von Ethan-1,2, Propan-1,3-, Butan-1,4- und Pentan-1,5-diol liegen die Ausbeuten an einfach geschützten Produkten zwischen 85 und $95 \%{ }^{[75]}$

Die Anwendung dieser Vorschrift auf das Diol 8 ergibt den monosilylierten Alkohol rac-52 mit einer Ausbeute von 83 \%. Die bissilylierte Verbindung wurde nicht gebildet.

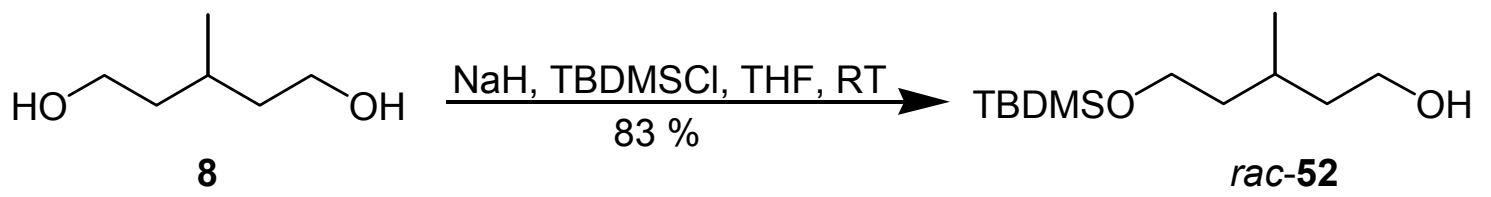

Die Transformation des geschützten Alkohols ins Iodid rac-6 gelingt in sehr guten Ausbeuten auf etabliertem Weg durch Mesylierung der Alkoholfunktion und Verdrängung der guten Abgangsgruppe mit Lithiumiodid in siedendem Aceton oder Tetrahydrofuran. 


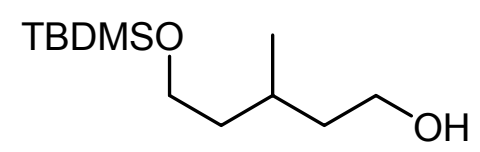

rac-52
1. $\mathrm{CH}_{2} \mathrm{Cl}_{2}, \mathrm{NEt}_{3}, \mathrm{CH}_{3} \mathrm{SO}_{2} \mathrm{Cl}, 0{ }^{\circ} \mathrm{C}$

2. Nal, THF, $66^{\circ} \mathrm{C}$.

$93 \%$

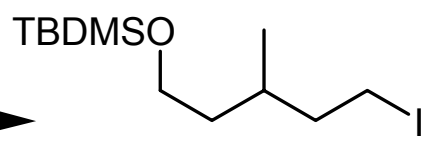

rac-6

Dem Solvens Tetrahydrofuran ist dabei der Vorzug $\mathrm{zu}$ geben, denn Versuche mit Natriumiodid unterschiedlicher Qualität führten unter den oben angegebenen Bedingungen mit Aceton als Lösungsmittel wiederholt zur Spaltung des Silylethers. Die Umsetzung derselben Reaktionspartner in siedendem, trockenem Tetrahydrofuran verlief dagegen problemlos und ebenfalls mit sehr guter Ausbeute.

Versuche zur Synthese des entsprechenden Bromids rac-53 durch den Umsatz von rac-52 mit Lithiumbromid in siedendem Tetrahydrofuran ergaben deutlich schlechtere Ergebnisse. Stets entstand entschütztes Nebenprodukt, was auf die Lewis-Säure-Aktivität von Lithiumbromid zurückzuführen ist.

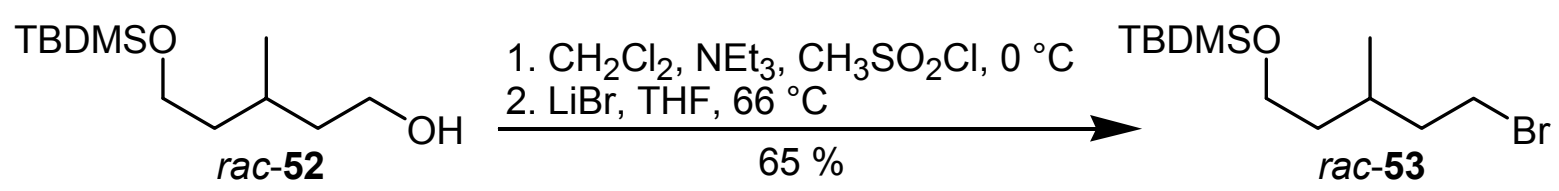

Mit der dreistufigen Synthese von rac-6 in $77 \%$ Gesamtausbeute stand einer der für die Synthese von $r a c-1$ benötigten Vorläufer zur Verfügung.

\subsection{Synthese von $(R)-6$}

Der in hoher optischer Reinheit (ee > 95\%) im Handel erhältliche (R)-3-Methylglutarsäuremonoethylester $[(R)-11]$ sollte durch die Anwendung einer stereodivergenten Synthesestrategie als Substrat sowohl für die Synthese von $(R)-6$ als auch für die des Enantiomers $(S)-6$ eingesetzt werden können.

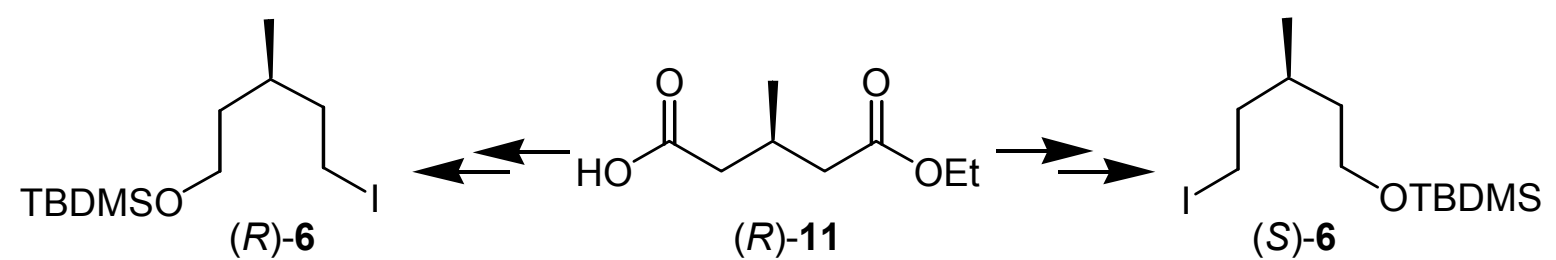

Demnach müßte es möglich sein, ausgehend von $(R)$-11 beide Antipoden des Naturstoffs Methanophenazin $(S)-\mathbf{1}$ und $(R)-\mathbf{1}$, aufzubauen. 
<smiles>CC(C)=CCC/C(C)=C/CC/C(C)=C/CC/C(C)=C/CC[C@H](C)CCOc1ccc2nc3ccccc3nc2c1</smiles>

$(S)-1$<smiles>CC(C)=CCC/C(C)=C/CC/C(C)=C/CC/C(C)=C/CC[C@H](C)CCOc1ccc2nc3ccccc3nc2c1</smiles>

Eine gesättigte $\mathrm{C}_{5}$-Kette, die an C-3 ein Stereozentrum mit einer Methylgruppe trägt, ist Strukturelement vieler Pheromone ${ }^{[76]}$ Terpene $^{[77]}$ und anderer natürlich vorkommender Verbindungen. Damit sind chirale Substanzen wie $(R)-\mathbf{1 1}$ begehrte Bausteine für die Synthese von Verbindungen wie $\alpha$-Tocopherol ${ }^{[78]}$ oder Verrucarinsäure. ${ }^{[79]}$

Die Gewinnung des mittlerweile im Handel erhältlichen $(R)$-11 erfolgt auf zwei prinzpiell unterschiedliche Art und Weisen. Bei der klassischen Methode der Racematspaltung durch Kristallisation diastereomerer Salze verwendet man das enantiomerenreine Alkaloid Cinchonidin als Base und setzt es mit racemischen Glutarsäureethylester (rac-54) zum entsprechenden Salz um. Kristallisation und die Freisetzung des Alkaloids in saurem Medium ergibt die chirale Verbindung $(R)-\mathbf{1 1} .^{[80]}$<smiles>CCOC(=O)CC(C)CC(=O)O</smiles>

rac-54
1. Cinchonidin, $\mathrm{H}_{2} \mathrm{O}$

2. Kristallisation

3. $\mathrm{HCl}, \mathrm{H}_{2} \mathrm{O}$<smiles>CCOC(=O)CC(C)CC(=O)O</smiles>

(R)-11

Ein eleganter Ansatz zur Gewinnung chiraler Verbindungen aus prochiralen Vorläufern nutzt die Fähigkeit von Enzymen wie $\alpha$-Chymotrypsin, ${ }^{[81]}$ Schweineleber-Esterase $^{[82]}$ und Pferdeleber-Alkoholdehydrogenase ${ }^{[83]}$ enantiotopdifferenzierende Reaktionen zu katalysieren. So wird der prochirale Diester 55 durch das Enzym Schweineleber-Esterase in sehr guten Enantiomerenüberschüssen in $(R)$-11 übergeführt. Das Enzym stellt in dieser Reaktion einen biologischen Katalysator dar, der in der Lage ist, die beiden enantiotopen Liganden des Prochiralitätszentrums zu differenzieren und damit selektiv die Hydrolyse des pro- $R$ Ethylesters von $\mathbf{5 5}$ zu ermöglichen. 
<smiles>CCOC(=O)CC(C)CC(=O)OCC</smiles>

55

(R)-11

Diese Methode hat gegenüber der o. g. klassischen Racematspaltung den Vorteil, daß das gesamte Substrat in ein einziges Enantiomer umgewandelt werden kann.

Die ersten Schritte der Synthese des Iodids $(R)-\mathbf{6}$ sahen vor, $(R)-\mathbf{1 1}$ durch chemoselektive Reduktion die Carboxylgruppe in den entsprechenden Alkohol überzuführen und zu silylieren. ${ }^{[84]}$

Bei dem Versuch diese Transformationen durchzuführen, stellten sich gleich zu Beginn Schwierigkeiten ein. Während die Reduktion der Carboxylgruppe durch Diboran $\left(\mathrm{BH}_{3} \cdot \mathrm{SMe}_{2}\right)$ problemlos gelang, ${ }^{[85]}$ bereitete der Schutz des gebildeten Alkohols als Silylether Probleme. Im basischen Medium konkurriert die Silylierung mit der gleichzeitig ablaufenden Lactonisierung. Dabei ist der intramolekulare Prozeß gegenüber dem intermolekularen im Vorteil, so daß überwiegend oder ausschließlich das Lacton entsteht. ${ }^{[86]}$ Der Versuch, die Lactonbildung durch den Einsatz von drei Äquivalenten tert.-Butyldimethylsilylchlorid zu verhindern, war erfolglos. Der cyclische Ester blieb Hauptprodukt der Umsetzung. Um dieses Problem zu lösen, versuchte man zunächst das Lacton $(R)-\mathbf{1 2}$ zu synthetisieren und dieses dann in das Iodid $(R)-6$ zu überführen.

\subsubsection{Synthese der Lactone $(R)-12$ und $(S)-12$}

Carbonsäuren und Carbonsäureester werden mit komplexen Metallhydriden üblicherweise zu Alkoholen reduziert. Die selektive Reduktion einer Carbonsäuregruppe in Gegenwart eines Esters gestattet das Reagenz Diboran $\left(\mathrm{B}_{2} \mathrm{H}_{6}\right)$. In Form seines Dimethylsulfidkomplexes $\mathrm{BH}_{3} \cdot \mathrm{SMe}_{2}$ ist es in seiner Reaktivität genügend abgeschwächt und kann auch im Überschuß eingesetzt werden, ohne das man Gefahr läuft, neben der Carboxylgruppe die Estergruppe zu reduzieren. ${ }^{[87]}$ Das Substrat darf jedoch keine Doppel- oder Dreifachbindungen enthalten, da diese durch $\mathrm{BH}_{3} \cdot \mathrm{SMe}_{2}$ hydroboriert werden können.

Die Reduktion der Carboxylgruppe wird nach der Zugabe von $\mathrm{BH}_{3} \cdot \mathrm{SMe}_{2}$ zum Substrat $(R)-\mathbf{1 1}$ bei Raumtemperatur durchgeführt. Die Reaktionsmischung wird am Ende mit Wasser behandelt, um den entstandenen Borsäureester zu hydrolysieren. Um eine quantitative 
Cyclisierung des intermediär entstehenden 5-Hydroxycarbonsäureesters zum Lacton $(R)$-12 zu erreichen, erhitzt man das Rohprodukt, in Gegenwart von $p$-Toluolsulfonsäure, mehrere Stunden mit Benzol am Wasserabscheider. ${ }^{[88]}$ Eine Alternative zur sauer katalysierten Cyclisierung bietet die im Rahmen dieser Arbeit entwickelte basische Cyclisierung des Rohprodukts mit wäßriger Kaliumhydroxidlösung. Um gute Ausbeuten an $(R)-\mathbf{1 2}$ zu erzielen, ist es wichtig, die aus zwei Phasen bestehende Mischung aus Rohprodukt und wäßriger Kaliumhydroxidlösung durch die Zugabe von Methanol zu homogenisieren. Vorteil des hier vorgestellten Verfahrens ist die hohe Ausbeute des benötigten Lactons $(R)-\mathbf{1 2}$.<smiles>CCOC(=O)CC(C)CC(=O)O</smiles>

$(R)-11$

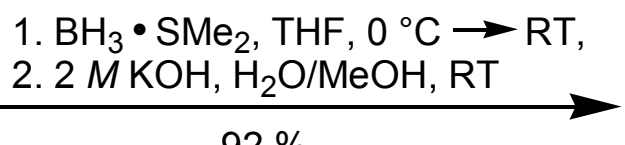

$92 \%$<smiles>C[C@H]1CCOC(=O)C1</smiles>

$(R)-12$

Der spezifische Drehwert $[\alpha]_{D}^{20}=+27.0^{\circ}\left(c=1.0\right.$ in $\left.\mathrm{CHCl}_{3}\right)$ von $(R)-\mathbf{1 2}$ entspricht auf Basis der Ergebnisse von Rossi ${ }^{[88]}\left\{\right.$ Lit. $[\alpha]_{D}^{27}=27.61^{\circ}\left(c=5.716\right.$ in $\left.\left.\mathrm{CHCl}_{3}\right)\right\}$ einem Enantiomerenüberschuß von ee $=98 \%$.

Die chemoselektive Reduktion der Esterfunktion in $(R)-\mathbf{1 1}$ gelingt nach einer Vorschrift von Dugar und seinen Mitarbeitern, ${ }^{[90]}$ mit Natriumborhydrid und Methanol in siedendem Dimethoxyethan. Die anschließende Lactonisierung mit wäßriger Kaliumhydroxidlösung liefert das Lacton $(S)-\mathbf{1 2}$ mit $84 \%$ Ausbeute.<smiles>CCOC(=O)CC(C)CC(=O)O</smiles>

$(R)-11$
1. $\mathrm{LiBH}_{4}, \mathrm{MeOH}, \mathrm{DME}, 85^{\circ} \mathrm{C}$ 2. $2 \mathrm{M} \mathrm{NaOH}, \mathrm{RT}$

$84 \%$<smiles>CC1CCOC(=O)C1</smiles>

(S)-12

Der spezifische Drehwert $[\alpha]_{D}^{20}=-24.0^{\circ}\left(c=1.0\right.$ in $\left.\mathrm{CHCl}_{3}\right)$ von Lacton $(S)$-12 entspricht, verglichen mit den Werten von Nohira ${ }^{[91]}\left\{\right.$ Lit. $[\alpha]_{D}^{20}=-27.0^{\circ}\left(c=1\right.$ in $\left.\left.\mathrm{CHCl}_{3}\right)\right\}$, einem Enantiomerenüberschuß von ee $=89 \%$. Die hier vorgestellten Resultate sind das Ergebnis erster orientierender Versuche. Um bessere Ergebnisse zu erzielen und das Potential der Methode auszuschöpfen, sind weitere Versuche zur Optimierung der Reaktionsbedingungen erforderlich. Für die weitere Synthese wurde das Lacton $(R)-\mathbf{1 2}$ als Startmaterial eingesetzt. 


\subsection{2 Öffnung des Lactons (R)-12 zum Amid (R)-56}

Die ersten Untersuchungen zur Öffnung von $(R)-\mathbf{1 2}$ wurden mit 60 proz. und 80 proz. wässriger Dimethylaminlösung bei Temperaturen von 100 bis $120{ }^{\circ} \mathrm{C}$ im Druckkolben durchgeführt. ${ }^{[91]}$ Neben dem entsprechenden Amid wurden nach $48 \mathrm{~h}$ im ${ }^{1} \mathrm{H}$ - und ${ }^{13} \mathrm{C}$ NMRSpektrum noch etwa 10 bis $20 \%(R)$-12 detektiert. Versuche bei höheren Temperaturen als $120{ }^{\circ} \mathrm{C}$ führten zur Zersetzung des Substrats $(R)$-12. Die angestrebte Bildung eines Hydroxycarbonsäureamids wurde schließlich durch zwölfstündiges Erhitzen des Lactons $(R)-\mathbf{1 2}$ mit Piperidin bei $70{ }^{\circ} \mathrm{C}$ erreicht. Das stabile Piperidid $(R)-56$ entsteht dabei mit $98 \%$ Ausbeute nahezu quantitativ.

$\overbrace{0} \frac{\text { Piperidin, } 12 \mathrm{~h}, 70^{\circ} \mathrm{C}}{98 \%}$

$(R)-12$<smiles>C[C@H](CCO)CC(=O)N1CCCCC1</smiles>

$(R)-56$

\subsubsection{Diskussion der spektroskopischen Daten von $(R)-56$}<smiles>C[C@@H](CCO)CC(=O)N1[CH]CCC[CH]1</smiles>

Im IR-Spektrum von $(R)-56$ zeugt eine breite Bande bei $\widetilde{v}=3406 \mathrm{~cm}^{-1}$ von Wasserstoffbrückenbindungen, deren Ursache die primäre Alkoholgruppe des Moleküls ist. Neben den symmetrischen und asymmetrischen Streckschwingungen der aliphatischen $\mathrm{CH}_{2}-\mathrm{Gruppen}$ mit Absorptionsbanden bei $\widetilde{v}=2933$ und $2857 \mathrm{~cm}^{-1}$ ist die Carbonylschwingungsbande der Amidgruppe bei $\widetilde{v}=1620 \mathrm{~cm}^{-1}$ gut zu identifizieren. 
Im ${ }^{1} \mathrm{H}$ NMR-Spektrum erscheint das Signal der Methylgruppe durch eine ${ }^{3} J$-Kopplung mit dem 3-H Methin-Proton als Dublett bei $\delta=0.95 \mathrm{ppm}$. Das Hydroxylproton resoniert in Form eines breiten Singuletts bei $\delta=3.16 \mathrm{ppm}$. Die unmittelbare Nähe des elektronegativen Stickstoffs entschirmt die Methylenprotonen der Kohlenstoffatome C-1' und C-5' und verschiebt ihre Resonanzen $\mathrm{zu} \delta=3.37$ und $3.51 \mathrm{ppm}$. Am stärksten zu tiefem Feld verschoben sind die Resonanzen der diastereotopen 5- $\mathrm{H}_{2}$ Protonen. Das ihnen zugeordnete Signal erscheint bei $\delta=3.57 \mathrm{ppm}$.

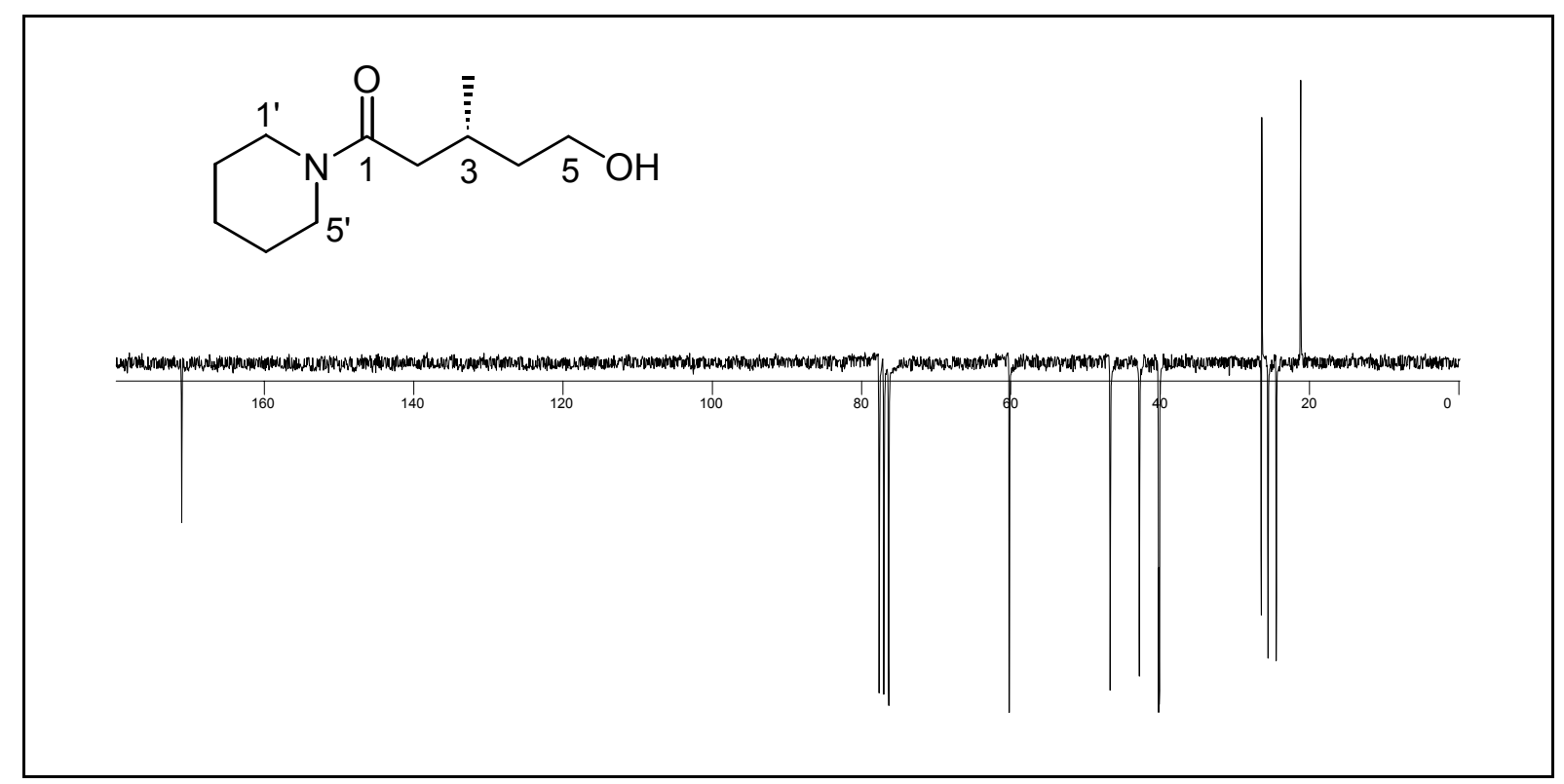

Abb. 41: ${ }^{13} \mathrm{C}$ APT NMR-Spektrum von $(R)-56\left(50 \mathrm{MHz}, \mathrm{CDCl}_{3}\right)$

Im ${ }^{13} \mathrm{C}$ NMR-Spektrum (Abbildung 41) resoniert die Methylgruppe bei $\delta=21.04 \mathrm{ppm}$. Eng beieinander und im einzelnen nicht genau zuzuordnen sind die Signale der Atome C-2', C-3' und C-4', die im Bereich von $\delta=24.43$ bis 26.44 ppm in Erscheinung treten. Die C-NBindung besitzt aus Mesomeriegründen einen hohen Doppelbindungsanteil. Als Folge der eingeschränkten Drehbarkeit um diese Bindung befinden sich die Kohlenstoffatome des Piperidinrings C-1' und C-5' relativ zum Carbonylsauerstoff $(E)$ - bzw. (Z)-angeordnet. $\operatorname{Im}{ }^{13} \mathrm{C}$ NMR-Spektrum beobachtet man für diese Positionen unterschiedliche chemische Verschiebungen. Das zum Carbonylsauerstoff $(Z)$-angeordnete $\alpha$-Kohlenstoffatom des tertiären Amid-Stickstoffs resoniert bei höherem, das $(E)$-angeordnete C-Atom resoniert bei tieferem Feld. Deshalb wird das Signal bei $\delta=42.82 \mathrm{ppm}$ dem Atom C-1', das Signal bei $\delta=$ 
46.75 ppm dem Kohlenstoffatom C-5' zugeordnet. Die Resonanzen der C-Atome der Hydroxylgruppe und der Amidgruppe finden sich bei $\delta=60.13$ bzw. 171.16 ppm.

\subsubsection{Reduktion des Piperidids $(R)-56$ und Synthese von $(R)-6$}

Das Hydroxyamid $(R)$-56 kann durch Silylierung der Hydroxylgruppe mittels tert.Butyldimethylsilylchlorid und Triethylamin mit $98 \%$ Ausbeute in Verbindung $(R)-57$ übergeführt werden.

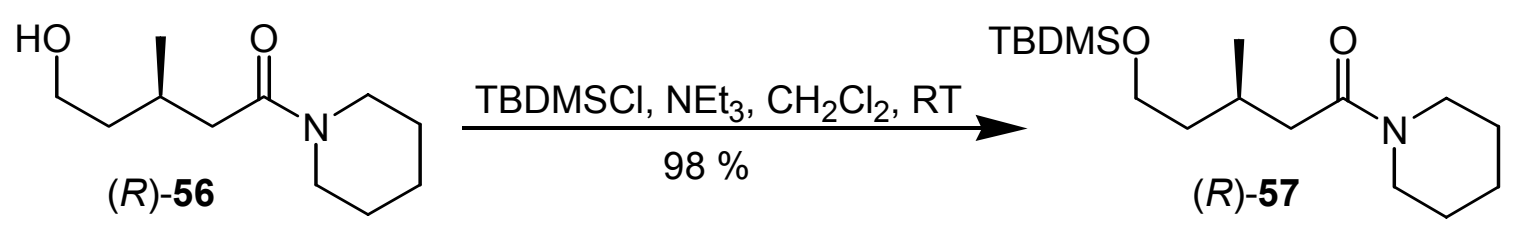

Die einstufige Reduktion von tertiären Amiden $\mathrm{zu}$ den korrespondierenden primären Alkoholen ist ein Spezialfall der selektiven Reduktion von Amiden. Die Addition eines Hydrid-Anions an die Carbonylgruppe eines tertiären Amids 58 führt im ersten Schritt zunächst zum Tetraeder-Intermediat 59, das dann durch Spaltung der C-N-Bindung zum primären Alkohol 60, bzw. durch Spaltung der C-O-Bindung zum tertiären Amin 61 zerfallen kann (Abbildung 42). ${ }^{[89]}$
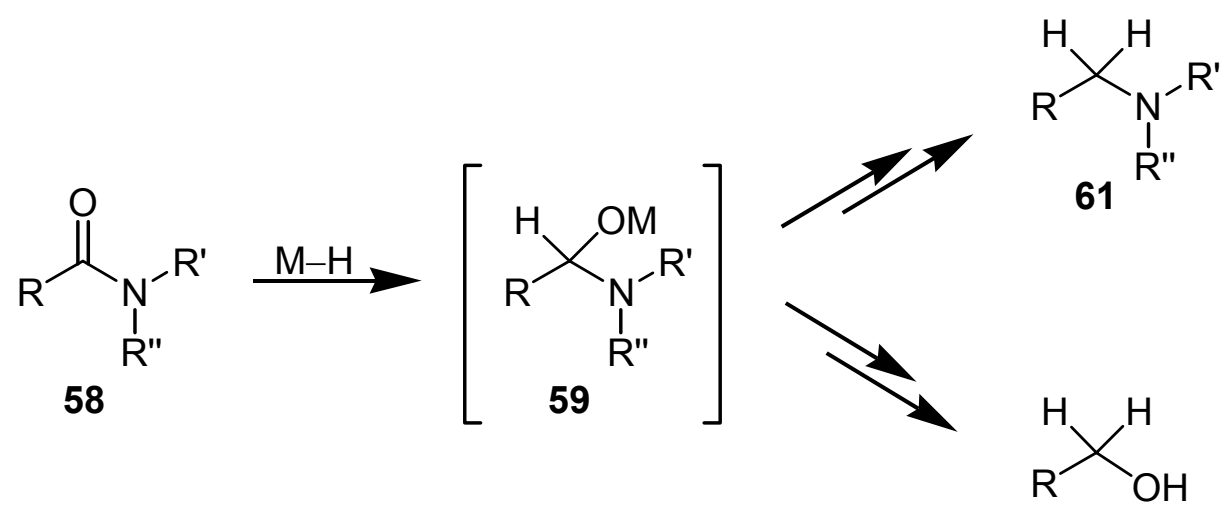

60

Abb. 42: Schema zur Reduktion von tertiären Amiden mit Metallhydriden (M-H)

Typische Metallhydride, wie Lithiumaluminiumhydrid $\left(\mathrm{LiAlH}_{4}\right)$ und Di-iso-butylaluminiumhydrid (DIBAL), reagieren bevorzugt unter Bildung der Amine. Wichtige Ausnahmen bilden das von H. C. Brown und seinen Mitarbeitern ${ }^{[92]}$ entwickelte Lithiumtriethylborhydrid 
$\left(\operatorname{LiEt}_{3} \mathrm{BH} ;\right.$ Superhydride ${ }^{\circledR}$ ), sowie das von B. Singaram und Mitarbeitern ${ }^{[93]}$ eingeführte Lithiumpyrrolidin-Boran-Reagenz $\left[\mathrm{Li}\left(\mathrm{CH}_{2}\right)_{4} \mathrm{NBH}_{3} ;\right.$ LPT]. Neben diesen Verbindungen, entwickelten $A$. G. Myers und seine Mitarbeiter ${ }^{[94]}$ Lithiumamidotrihydroborat $\left(\mathrm{LiH}_{2} \mathrm{NBH}_{3}\right.$; LAB) als Reagenz zur selektiven Reduktion von Pseudoephedrinamiden zu primären Alkoholen.

Das prominenteste der oben aufgeführten Reduktionsmittel ist Lithiumtriethylborhydrid. Es wird in der Regel zur Reduktion von $N, N$-Dimethylamiden eingesetzt und hat sich dort erfolgreich bewährt. Die Ausbeuten dieser Reaktion liegen meistens im Bereich zwischen 90 und $100 \%$. Untersuchungen an höher substituierten Amiden, wie beispielweise an Propansäurediethylamid und an Propansäurepyrrolidid zeigen eine deutliche Abnahme der Ausbeuten mit zunehmender Komplexität der Amide. ${ }^{[92]}$ Im Fall der Reduktion des Propansäurepyrrolidids wurde der entsprechende Alkohol, das 1-Propanol, mit nur $50 \%$ Ausbeute erhalten.

Über die einstufige Reduktion eines Piperidids zu einem Alkohol wurden in der Literatur keine Informationen gefunden. Es war deshalb umso erfreulicher, daß die Umwandlung von (R)-57 zum Alkohol (S)-52 mit $\mathrm{LiEt}_{3} \mathrm{BH}$ als Reduktionsmittel problemlos verlief. Die rasch und unter milden Bedingungen verlaufende Reaktion und ergab das Produkt $(S)$-52 in $92 \%$ Ausbeute.

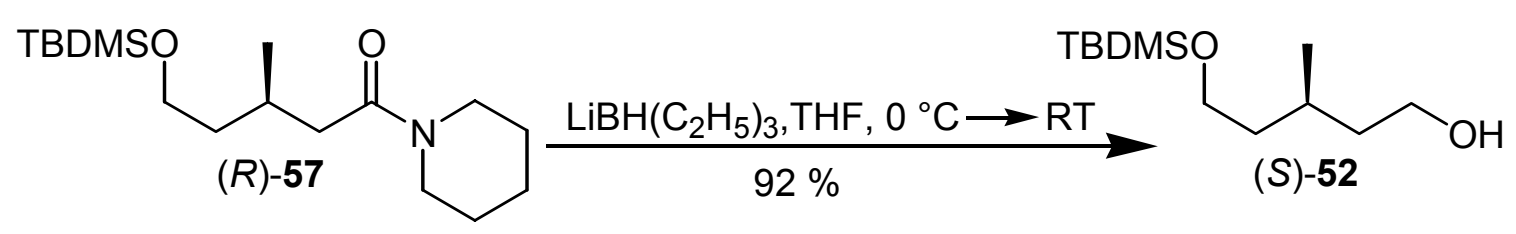

Die abschließende Transformation des Silyloxyalkohols $(S)-52$ in den chiralen $\mathrm{C}_{6}$-Baustein (R)-6 wurde analog zur Synthese von rac-6 (Kapitel 8.1), durch Aktivieren der Hydroxylgruppe als Mesylat und deren Substitution durch Iodid in siedendem Tetrahydrofuran erfolgreich durchgeführt.

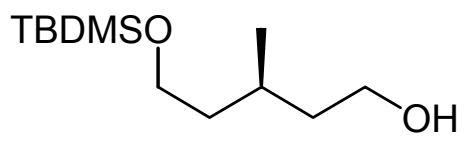

(S) -52

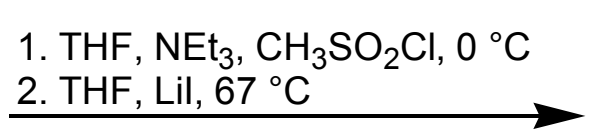

$93 \%$

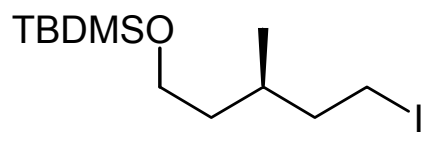

(R)-6 


\subsubsection{Diskussion der spektroskopischen Daten von $(R)-6$}

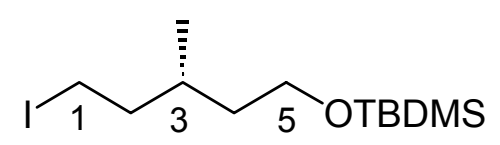

$(R)-6$

Im IR-Spektrum prägen mehrere Absorptionsbanden der C-H-Streckschwingungen den Bereich von $\widetilde{v}=2955$ bis $2857 \mathrm{~cm}^{-1}$. Hinweise auf den Silylether geben die Absorptionsbanden bei $\widetilde{v}=1098$ und $836 \mathrm{~cm}^{-1}$.

Das ${ }^{1} \mathrm{H}$ NMR-Spektrum enthüllt durch charakteristische Resonanzsignale bei $\delta=0.03$ und $0.87 \mathrm{ppm}$ die Anwesenheit einer tert.-Butyldimethylsilyl-Gruppe. Sie überlagern das Dublett bei $\delta=0.87 \mathrm{ppm}$, das durch die Methylgruppe am Stereozentrum hervorgerufen wird. Die diastereotopen 2- $\mathrm{H}_{2^{-}}, 3-\mathrm{H}-$ und 4- $\mathrm{H}_{2}$-Protonen führen zu komplexen Resonanzen im Bereich von $\delta=1.34$ bis $1.97 \mathrm{ppm}$ und lassen sich nur im Einzelnen schwer zuordnen. Der negative induktive Effekt von Sauerstoff- und Iodatom lassen die Signale der Protonen 1- $\mathrm{H}_{2}$ und 5- $\mathrm{H}_{2}$ bei $\delta=3.15$ und 3.23 , bzw. bei 3.60 und 3.65 ppm in Erscheinung treten.

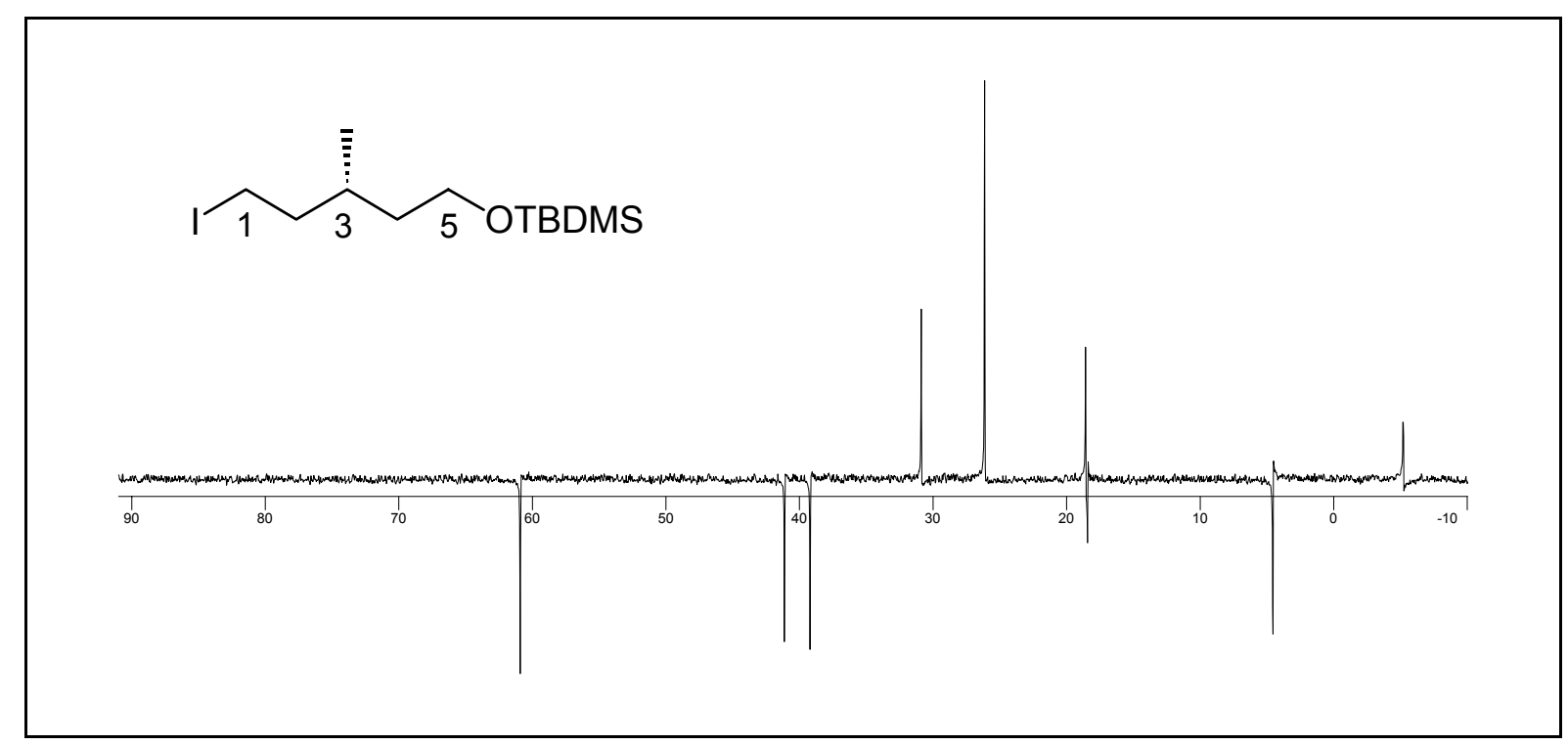

Abb. 43: ${ }^{13} \mathrm{C}$ APT NMR-Spektrum von $(R)-6\left(50 \mathrm{MHz}, \mathrm{C}_{6} \mathrm{D}_{6}\right)$

Im ${ }^{13} \mathrm{C}$ NMR-Spektrum (Abbildung 43) erkennt man die Resonanzen der Kohlenstoffatome, die direkt mit dem Silicium- bzw. mit dem Iodatom verbunden sind, an ihrer charakteristischen Hochfeldverschiebung. Sie resonieren bei $\delta=-5.29$ sowie -5.26 
$\left[\left(\mathrm{SiCH}_{3}\right)_{2}\right]$ und 4.91 (C-1) ppm. Das Signal des quartären Kohlenstoffatoms der tert.Butylgruppe bei $\delta=18.42 \mathrm{ppm}$ läßt sich im ${ }^{13} \mathrm{C}$ APT NMR-Spektrum durch seine entgegengesetzte Orientierung gut vom Signal der Methylgruppe am stereogenen Zentrum bei $\delta=18.54$ ppm unterscheiden. Das stärkste Signal erzeugen die drei Methylgruppen der tert.Butylgruppe bei $\delta=26.13 \mathrm{ppm}$. Das stereogene Atom C-3 resoniert bei $\delta=30.35 \mathrm{ppm}$ und die Signale der Atome C-2 und C-4 erscheinen bei $\delta=39.18$ und 41.07 ppm. Bedingt durch den elektronegativen Sauerstoff resoniert das Kohlenstoffatom C-5 bei $\delta \square=60.88$ ppm.

\subsection{Bestimmung der optischen Reinheit des $C_{6}$-Bausteins $(R)-6$}

Neben den spezifischen Drehwerten, deren Bestimmung mit einem relativ großen Fehler behaftet ist und über die in der Literatur oft uneinheitliche Angaben gemacht werden, wurden noch andere, voneinander unabhängige Methoden zur Bestimmung des Enantiomerenüberschusses angewendet. Eine praktikable Vorgehensweise ist die gaschromatographische Separation der Enantiomeren an chiraler Phase. Versuche, die Verbindungen rac-52 und rac6 an der chiralen stationären Phase Heptakis-(2,6-O-methyl-3-O-pentyl)- $\beta$-cyclodextrin in ihre Spiegelbildisomeren aufzutrennen, waren erfolglos.

Eine andere, ebenfalls häufig angewandte Methode zur Bestimmung des Enantiomerenüberschusses, ist die Umsetzung der Probensubstanz mit einem chiralen Derivatisierungsreagenz. Zur Derivatisierung von primären und sekundären Alkoholen ist die (nach seinem Erfinder) als Mosher-Reagenz bekannte $\alpha$-Methoxy- $\alpha$-(trifluormethyl)phenylessigsäure (62) das Mittel der Wahl. ${ }^{[95]}$ Sie ist sowohl als $(S)$ - als auch als $(R)$-Enantiomer kommerziell erhältlich.<smiles>C[C@@](C(=O)O)(c1ccccc1)C(F)(F)F</smiles>

$(S)-62$<smiles>C[C@](C(=O)O)(c1ccccc1)C(F)(F)F</smiles>

$(R)-62$ 
Die diastereomere Zusammensetzung der Probensubstanz wird nach ihrer Derivatisierung in der Regel durch eine Analyse der ${ }^{19}$ F NMR-Spektren bestimmt. Dies hat den Vorteil, daß die charakteristischen Signale der Fluoratome zur Diastereomerenbestimmmung nicht durch

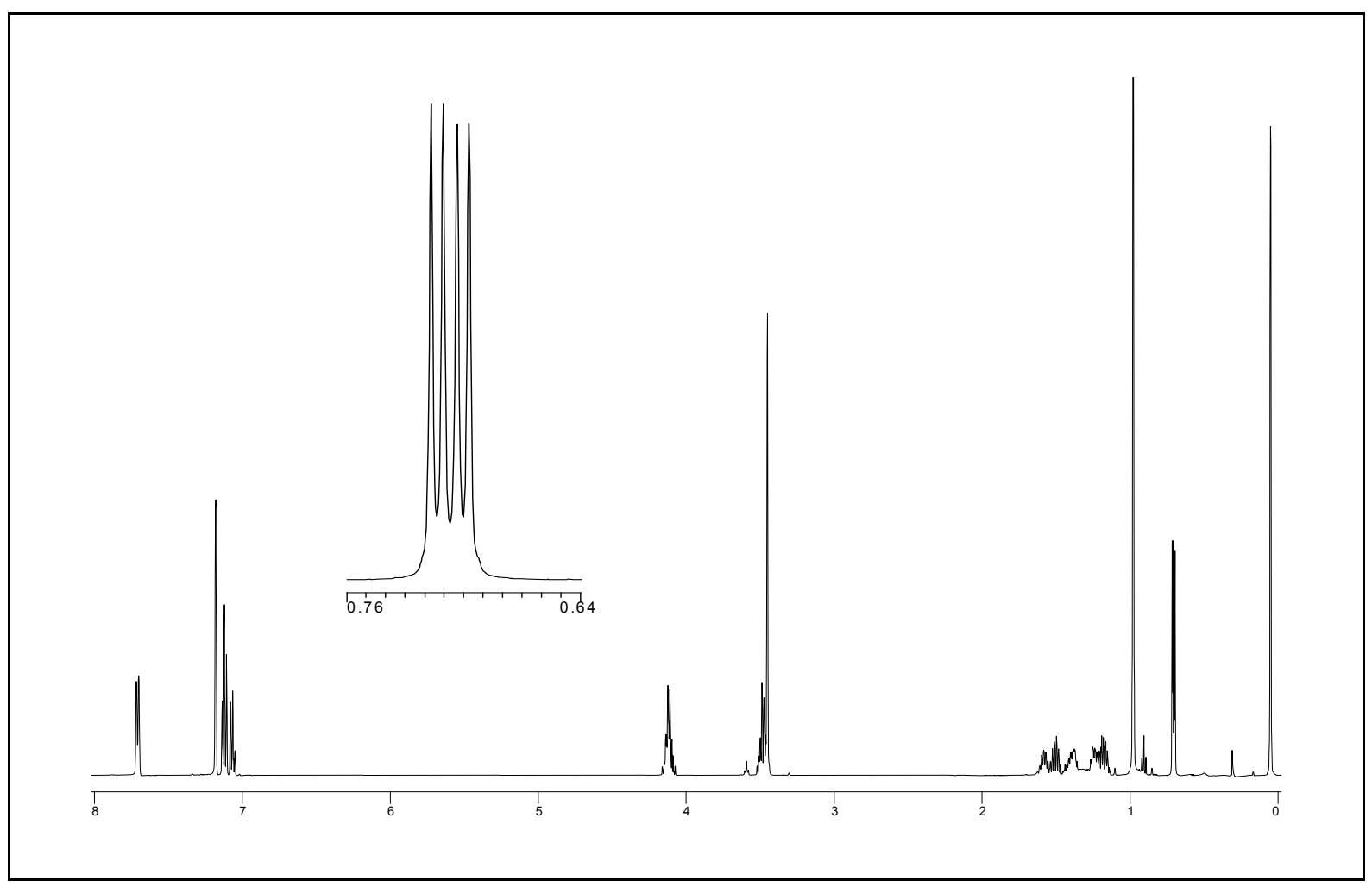

Abb. 44: ${ }^{1} \mathrm{H}$ NMR-Spektrum der diastereomeren Mosher-Ester $(S, S)-63$ und $(R, S)-63$ $\left(500 \mathrm{MHz}, \mathrm{C}_{6} \mathrm{D}_{6}\right)$; Diastereomerenverhältnis = $1: 1$

Resonanzen anderer Atome überlagert sind. Die Untersuchung der NMR-Spektren des durch Umsetzung von Verbindung rac-52 mit $(S)$ - 62 erhaltenen Diastereomerengemisches der Mosher-Ester $(S, S)-\mathbf{6 3}$ und $(R, S)$-63 ergaben allerdings weder im ${ }^{19} \mathrm{~F}$ - noch im ${ }^{13} \mathrm{C}$ NMRSpektrum Signale, die sich zur Bestimmung des Diastereomerenverhältnisses eigneten. 


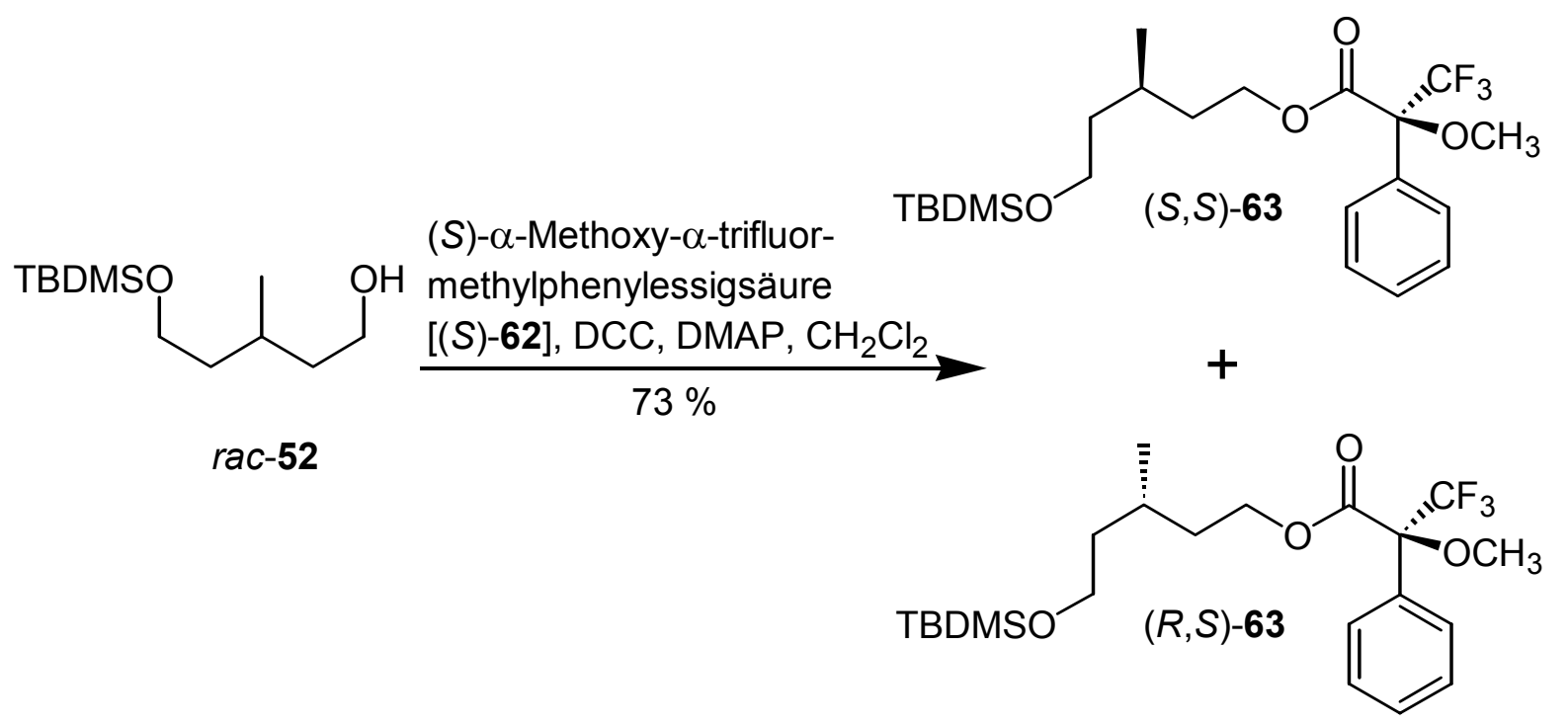

Dagegen zeigt die Aufnahme eines ${ }^{1} \mathrm{H}$ NMR-Spektrums der beiden Diastereomere $(S, S)-\mathbf{6 3}$ und $(R, S)-63$ in Deuterobenzol (Abbildung 44) eine ausreichend große Differenz in der chemischen Verschiebung der direkt am Stereozentrum befindlichen Methylgruppe $(\delta=0.705$ und $0.710 \mathrm{ppm}$ ). Sie kann deshalb zur Bestimmung des Diastereomeren- und damit des Enantiomerenverhältnisses herangezogen werden.

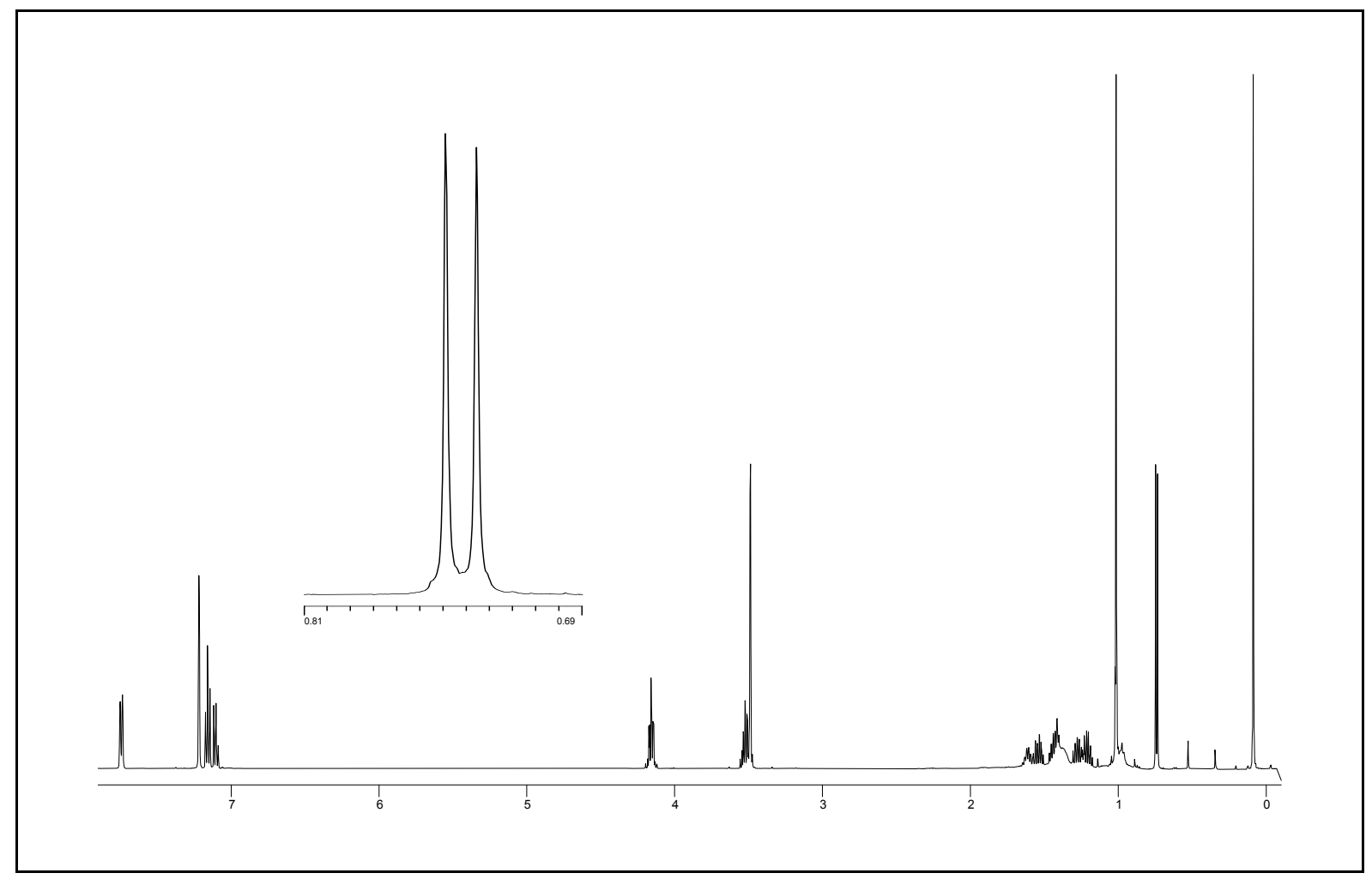

Abb. 45: ${ }^{1} \mathrm{H}$ NMR-Spektrum des Mosher-Esters (S,S)-63 (500 MHz, $\left.\mathrm{C}_{6} \mathrm{D}_{6}\right)$ 


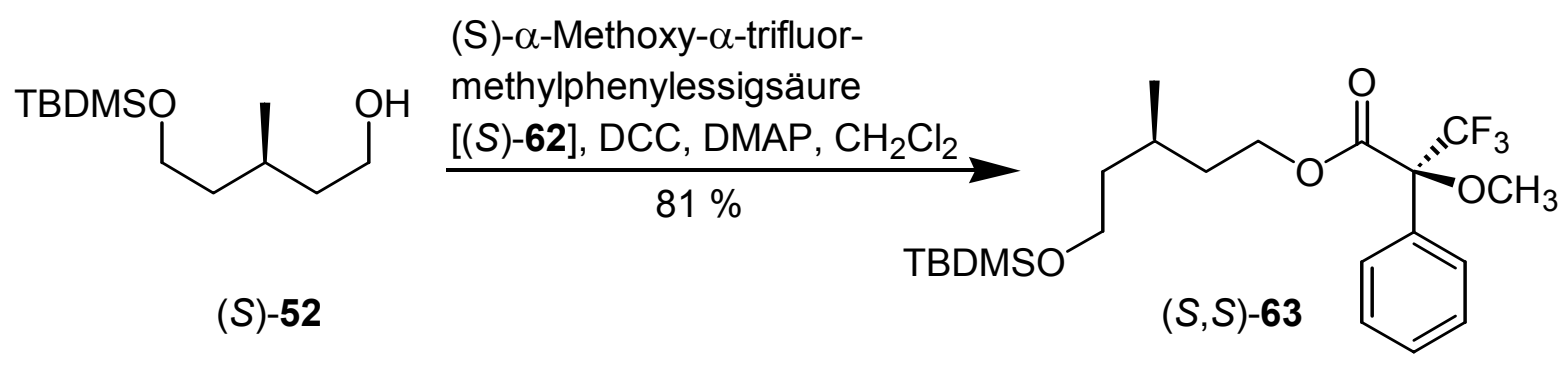

Das ${ }^{1} \mathrm{H}$ NMR-Spektrum des Produkts der Umsetzung von $(S)-\mathbf{5 2}$ mit $(S)-\mathbf{6 2}$ ist in Abbildung 45 dargestellt. Wie dem Spektrum zu entnehmen ist, läßt sich nur das $(S, S)$-63 Diastereomer nachweisen, da sich nur die Resonanz des Dubletts der Methylgruppe von $(S, S)$-63 bei $\delta=$ 0.745 ppm findet. Der Enantiomerenüberschuß des Alkohols $(S)$-52 ist damit größer als $95 \%{ }^{[107]}$

\subsection{Zusammenfassung}

Ausgehend von dem kommerziell erhältlichen, nahezu enantiomerenreinen chiralen $(R)-3$ Methylglutarsäureethylester $(R-11)$ (ee $>95 \%$ ) ist es möglich, durch chemoselektive Reduktion der Carbonsäure- bzw. der Esterfunktionalität die beiden enantiomeren Lactone $(S)-12$ und (R)-12 zu synthetisieren. $(R)$-12 erhielt man dabei mit einer Ausbeute von $92 \%$ und einem Enantiomerenüberschuß von 98 \%. Die Öffnung des Lactons $(R)$-12 mit Piperidin zum entsprechenden Hydroxyamid (R)-56 ergibt nach dem Silylieren der Hydroxylgruppe $(R)-57$. Die einstufige Reduktion des tertiären Amids $(R)-57$ zum primären Alkohol $(S)-52$, dessen Mesylierung und Umsetzung mit Kaliumiodid ergibt das benötigte Iodid $(R)-\mathbf{6}$.

Im Ergebnis gelingt die Synthese des enantiomerenreinen $\mathrm{C}_{6}$-Bausteins $(R)-6$ in Grammengen aus (R)-11 mit einer Gesamtausbeute von $76 \%$ über fünf Stufen. Die Bestimmung des Enantiomerenüberschusses auf der Stufe des Silyloxyalkohols $(S)$-52 nach der MosherMethode zeigt, daß die Integrität des Stereozentrums im Verlauf der Synthesesequenz unangetastet bleibt. 


\section{$9 \quad$ Synthese des diastereomerenreinen $\mathrm{C}_{19}$-Bausteins 7}

Im vorhergehenden Kapitel wurde die Synthese der gesättigten $\mathrm{C}_{6}$-Bausteine rac-6 und $(R)-\mathbf{6}$ ausführlich beschrieben. Zum Aufbau der kompletten Seitenkette von Methanophenazin (1) sah der Syntheseplan vor, rac-6 bzw. (R)-6 mit dem diastereomerenreinen Vinyliodid 7 zu verknüpfen (Abbildung 15). Es war geplant, diesen $\mathrm{C}_{19}$-Baustein durch eine Zirkoniumkatalysierte Carbometallierung eines entsprechenden terminalen Alkins 9 herzustellen. ${ }^{[96]}$ Als Startmaterial für die Synthese von 9 war das im Handel erhältliche, diastereomerenreine $(E, E)$-Farnesylaceton $(\mathbf{1 0})$ vorgesehen. ${ }^{[97]}$

\subsection{Synthese des terminalen Alkins 9}

Systematische Untersuchungen zur Chemie von Organozirkonium-Verbindungen durch Negishi und seine Mitarbeiter, führten zur Methode der Zirkonium-katalysierten Carboaluminierung terminaler Alkine. ${ }^{[96]}$ Damit ist es möglich, eine endständige Dreifachbindung wie in 64 regio- und stereoselektiv in die entsprechende (E)-2-Methyl-1-alkenylaluminiumVerbindung $\mathbf{6 5}$ überzuführen. Ausgehend von $\mathbf{6 5}$ eröffnen sich eine Reihe von Reaktionsmöglichkeiten, wie etwa die Alkylierung, die Acylierung, die Transmetallierung oder auch die konjugierte Addition, die unter Substitution des Aluminiumatoms und Retention der Konfiguration der Doppelbindung verlaufen. ${ }^{[98]}$

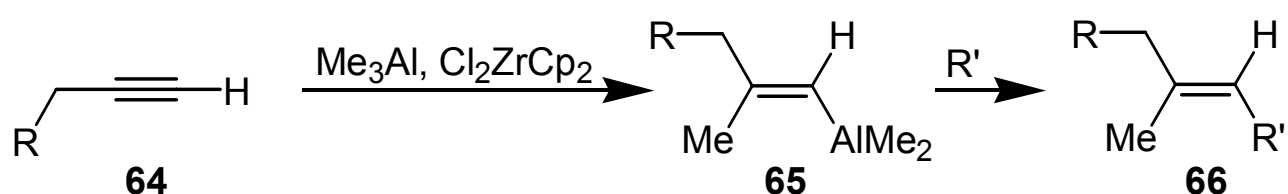

Die Methode eignet sich besonders gut zum Aufbau trisubstituierter-(E)-Olefine des Typs 66, einem bei Terpenen häufig auftretenden Strukturelement. Ihr Erfolg hängt entscheidend von einem effizienten Zugang zu dem entsprechenden terminalen Alkin ab. Erneut waren es Negishi und seine Mitarbeiter, ${ }^{[96,99,100]}$ die 1980 über eine breit anwendbare Methode zur Umwandlung von Methylketonen in endständige Dreifachbindungen berichteten.

Der erste Schritt dieser Transformation besteht in der Generierung des kinetischen Enolats durch Deprotonierung des Methylketons 67 bei $-78{ }^{\circ} \mathrm{C}$ mit einer sperrigen Base wie Lithiumdi-iso-propylamid (LDA) oder Lithium-2,2,6,6-tetramethylpiperidid (LiTMP) und dessen Abfangen mit Diethylchlorophosphat $\left[\mathrm{ClPO}(\mathrm{OEt})_{2}\right]$ als Phosphoenolether 68. Erneutes 
Deprotonieren mit 2 Äquivalenten LDA bzw. LiTMP in der Kälte führt zur $\beta$-Eliminierung von Diethylphosphat und ergibt das entsprechende Acetylid, welches bei der Aufarbeitung mit wäßriger Säure zum terminalen Alkin 69 protoniert wird.

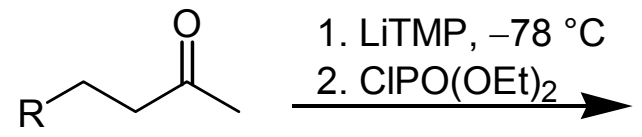

67

1. 2 Äquiv. LiTMP, $-78^{\circ} \mathrm{C}$

2. $\mathrm{HCl}, \mathrm{H}_{2} \mathrm{O}$<smiles></smiles>

68

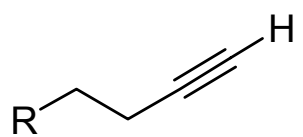

69

Die Reaktion wird in der Regel im Stile einer „Eintopf-Reaktion“ ohne Isolierung des Phosphoenolethers durchgeführt und ergibt die Alkine, je nach Substrat, mit Ausbeuten zwischen 61 und $85 \%$. $^{[100]}$<smiles>CC(=O)CC/C=C(/C)CC/C=C(/C)CCC=C(C)C</smiles>

10

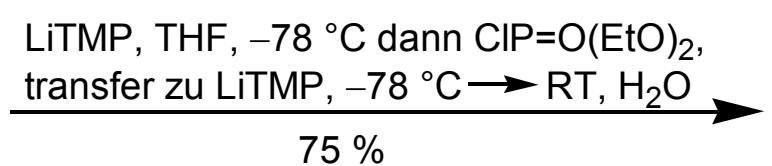

$75 \%$

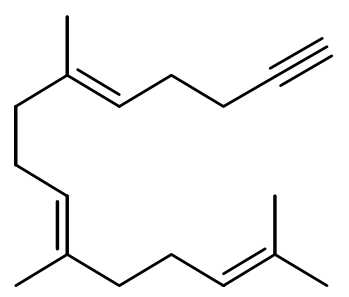

9

Durch die erfolgreiche Anwendung dieser Synthesemethode konnte $(E, E)$-Farnesylaceton (10) mit $75 \%$ Ausbeute in das terminale Alkin 9 umgewandelt werden. 


\subsection{Diskussion der spektroskopischen Daten von 9}

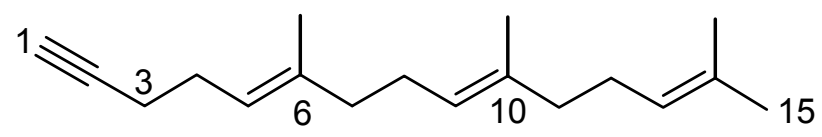

9

Das UV-Spektrum von 9 zeigt im Wellenlängenbereich $\lambda=190$ bis $400 \mathrm{~nm}$ keine Absorptionbanden, da die Doppelbindungen dieses Kohlenwasserstoffs untereinander nicht in Konjugation stehen.

Charakteristische Absorptionsbanden der terminalen Dreifachbindung treten im IR-Spektrum bei $\widetilde{v}=3311 \mathrm{~cm}^{-1}$ für die $\equiv \mathrm{C}-\mathrm{H}$ - und bei $\widetilde{v}=2119 \mathrm{~cm}^{-1}$ für die $\mathrm{C} \equiv \mathrm{C}-\mathrm{Streckschwingungen}$ auf. Im Bereich von $\widetilde{v}=2966$ bis $2854 \mathrm{~cm}^{-1}$ überlagern sich olefinische und aliphatische C-H-Schwingungsbanden. Die Absorption bei $\widetilde{v}=1667 \mathrm{~cm}^{-1}$ wird einer olefinischen $\mathrm{C}=\mathrm{C}$ Streckschwingung zugeordnet.

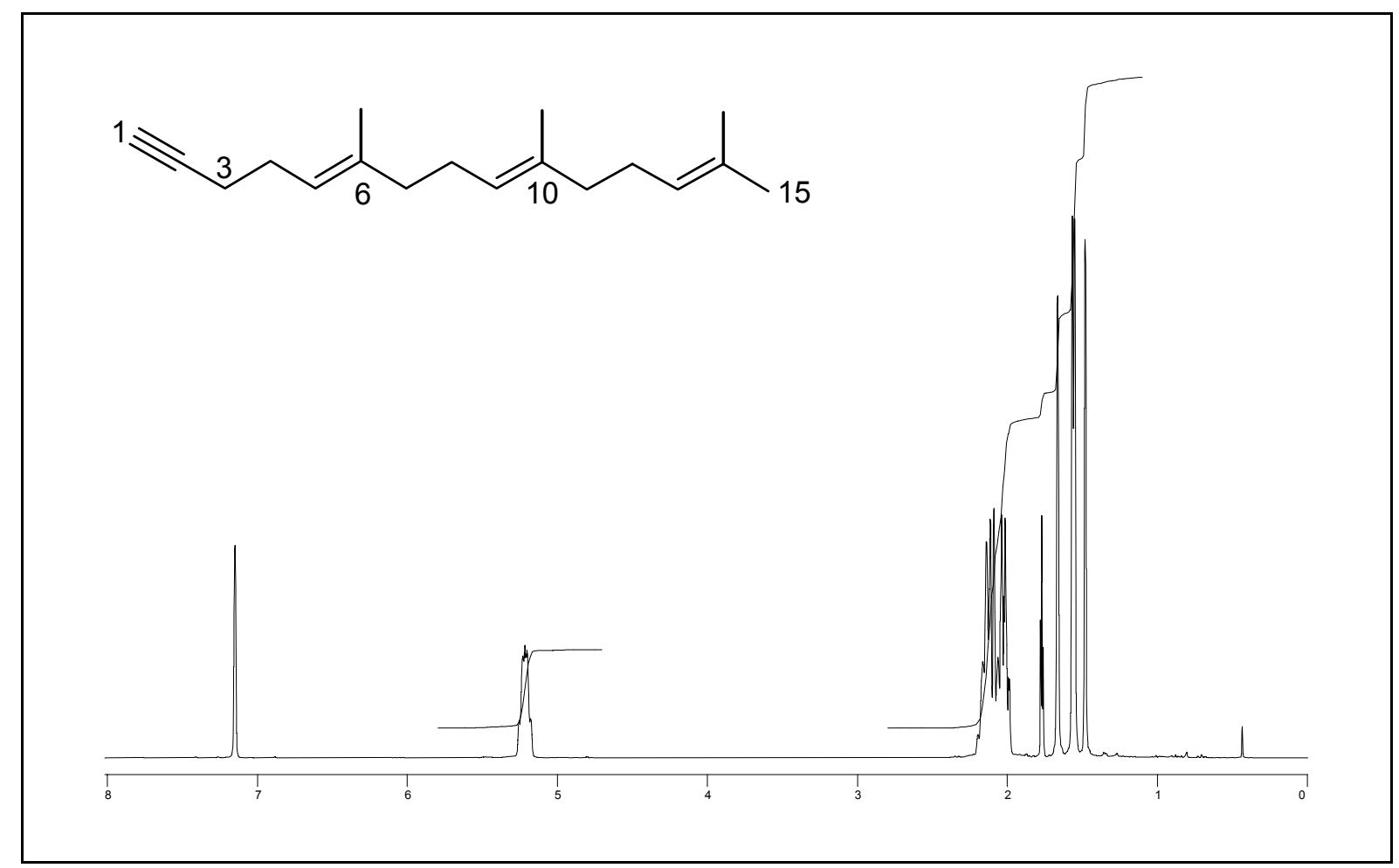

Abb. 46: ${ }^{1} \mathrm{H}$ NMR-Spektrum von Verbindung $9\left(300 \mathrm{MHz}, \mathrm{C}_{6} \mathrm{D}_{6}\right)$ 
Aufgrund sich wiederholender isoprenoider Strukturelemente im Molekül beobachtet man im ${ }^{1} \mathrm{H}$ NMR-Spektrum (Abbildung 46) zahlreiche sich überlagernde Signale. Dennoch gut zu identifizieren sind die vier olefinischen Methylgruppen bei $\delta=1.48,1.55,1.57$ und $1.66 \mathrm{ppm}$. Das Signal des acetylenischen Protons $1-\mathrm{H}$ erscheint durch eine ${ }^{4} J$-Kopplung mit den $3-\mathrm{H}_{2}$ Protonen als Triplett bei $\delta=1.77 \mathrm{ppm}$. Alle Methylenprotonen treten als breites, schwach aufgelöstes Signal zwischen $\delta=1.96$ und $2.22 \mathrm{ppm}$ in Erscheinung. Die vinylischen Protonen 5-H, 9-H, 13-H zeigen ein Multiplett zwischen $\delta=5.16$ und 5.28 ppm.

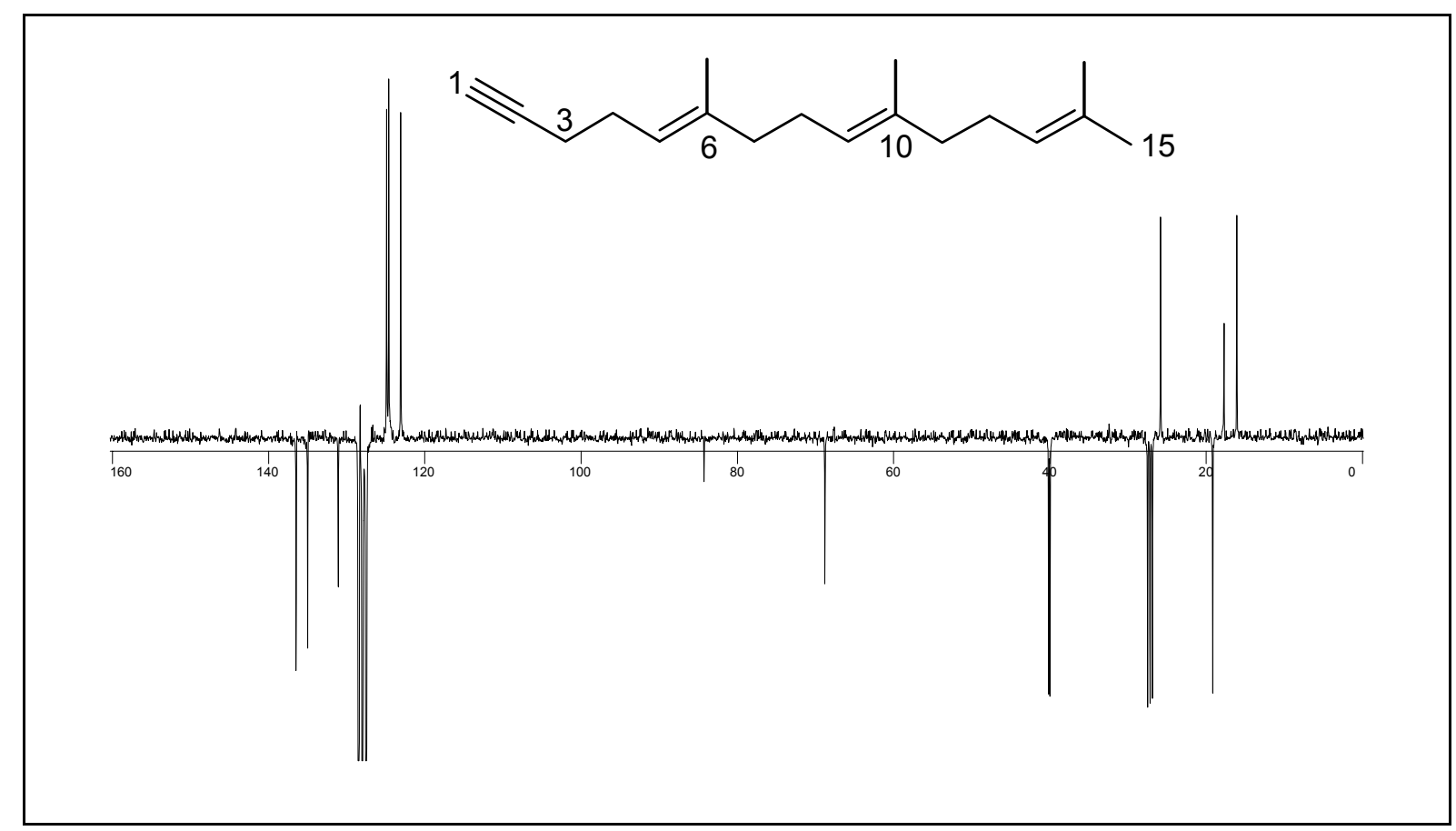

Abb. 47: ${ }^{13} \mathrm{C}$ APT NMR-Spektrum von Verbindung 9 (50 MHz, $\left.\mathrm{C}_{6} \mathrm{D}_{6}\right)$

Im ${ }^{13} \mathrm{C}$ NMR-Spektrum erscheinen die charakteristischen Signale der Kohlenstoffatome der Methylgruppen der Isopropylidengruppe von Verbindung 9 bei $\delta=17.72\left(14-\mathrm{CH}_{3}\right)$ und 25.48 (C-15) ppm (Abbildung 47). Neben den Resonanzen der Atome C-6 und C-10 bei $\delta=135.01$ und 136.49 ppm tritt das Signal von C-14 bei $\delta=131.09$ ppm in Erscheinung. Mit $\delta=68.81$ und 84.27 ppm liegen die Resonanzsignale der Kohlenstoffatome C-1 bzw. C-2 in einem für endständige Alkine typischen Bereich des ${ }^{13} \mathrm{C}$ NMR-Spektrums. Die vinylischen Atome C-5, C-9 und C-13 resonieren bei $\delta=123.09,124.63$ und 124.91 ppm. 


\subsection{Synthese des Vinyliodids 7}

Wie bereits in Abschnitt 9.1 dargestellt, ergibt die Reaktion eines terminalen Alkins 64 mit Trimethylaluminium (70) in Gegenwart von Zirkonocendichlorid (71) das entsprechende synAdditionsprodukt 65 mit einer Selektivität größer $95 \%$. Die $\mathrm{AlMe}_{2}$-Gruppe wird bei diesem Prozeß mit einer Regioselektivität von $95 \%$ auf das endständige Kohlenstoffatom von 64 übertragen, während die Methylgruppe an C-2 addiert. Die Ausbeuten für die anschließende Umsetzung der Alkenyl-Aluminium-Spezies 65 mit $\mathrm{I}_{2}$ zum Vinyliodid liegen im Bereich von 70 bis $85 \% .{ }^{[101]}$ Der genaue Mechanismus und die Gründe für den im hohen Maße regio- und stereoselektiv ablaufenden Vorgang sind in großen Teilen noch unverstanden. Man vermutet als Folge eines raschen Methyl-Chlor-Austauschs zwischen Zirkonocendichlorid (71) und Trimethylaluminium (70) die Bildung des Zirkon-Aluminium Komplexes 72, der mit der endständigen Dreifachbindung von 64 koordiniert (Abbildung 48). Den zentralen Schritt bildet die Addition der koordinativ ungesättigten Zirkon-Aluminium-Spezies $\mathbf{7 3}$ an die terminale Dreifachbindung von 64. ${ }^{[102]}$ Nach einem erneuten Methyl-Chlor-Austausch dissoziiert Zirkonocendichlorid $\mathbf{7 1}$ unter Freisetzung der 2-Methyl-1-alkenylaluminium Verbindung 65 aus dem Komplex 74 ab.

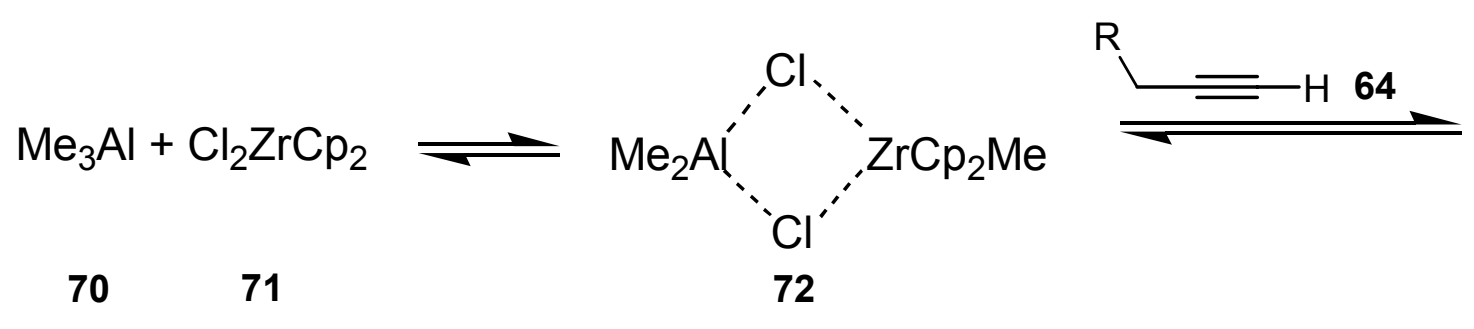

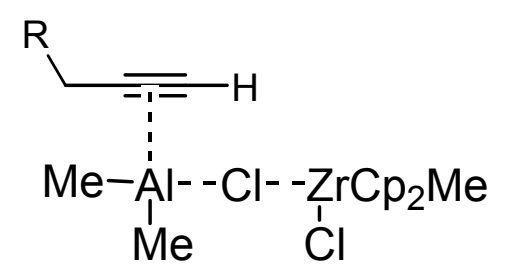

73

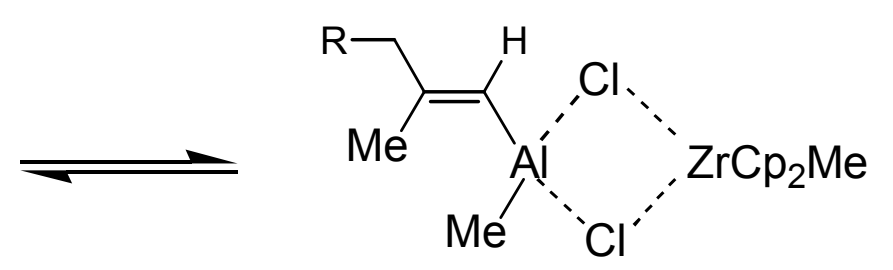

74

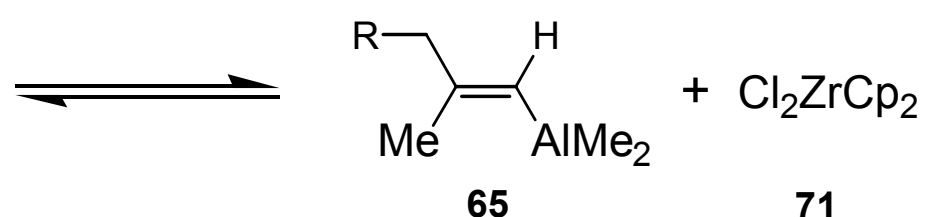

Abb. 48: Schema zum Mechanismus der Zirkonium-katalysierten Carboaluminierung terminaler Alkine 
Aus der Reaktion geht Zirkonocendichlorid (71) unverändert hervor und kann weitere Carboaluminierungs-Cyclen durchlaufen. Um gute Ausbeuten zu erzielen, verlangen die als „katalytisch“ bezeichneten Varianten dieser Synthesemethode in der Regel 20 bis 30 Molprozent Zirkonocendichlorid (71) sowie überstöchiometrische Mengen an Trimethylaluminium. Die Methode ist mit einigen funktionellen Gruppen, wie etwa der Hydroxylgruppe und den Halogenatomen Chlor, Brom und Iod sowie mit Silylschutzgruppen kompatibel und wurde in zahlreichen Naturstoffsynthesen eingesetzt. ${ }^{[98]}$

Zur Herstellung des Vinyliodids 7 wurden einer Vorschrift von Ley ${ }^{[103]}$ folgend 0.25 Äquivalente Zirkonocendichlorid (71) und drei Äquivalente Trimethylalminium (70) zur Carboaluminierung des diastereomerenreinem Alkins 9 eingesetzt. Die intermediär entstehende Alkenyl-Metall-Spezies wird durch Zugabe von elementarem Iod in Tetrahydrofuran zur Reaktionsmischung in 7 übergeführt. Die Ausbeuten der Reaktion liegen zwischen 68 und 74 \%. Der Einsatz von 0.5 Äquivalenten Zirkonocendichlorid (71) brachte in diesem Punkte keine Verbesserung.

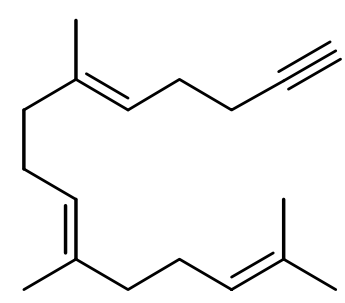

9

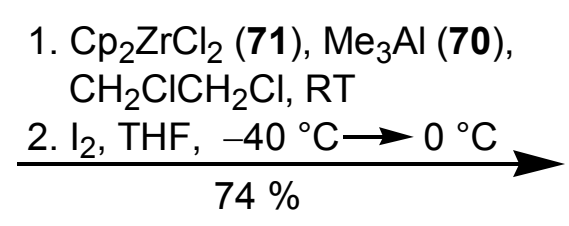
$\mathrm{CH}_{2} \mathrm{ClCH}_{2} \mathrm{Cl}, \mathrm{RT}$ $74 \%$

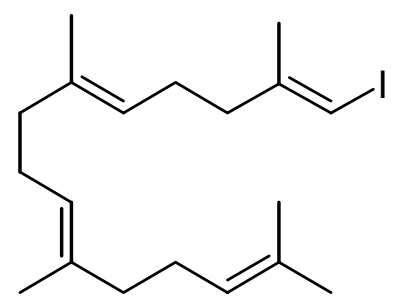

7

Die Reinigung des Rohprodukts an Kieselgel gestaltete sich aufwendig, da zur Abtrennung der unpolaren Nebenprodukte viel Adsorbens (Rohprodukt / Adsorbens $=1: 500$ ) und meist zweimaliges Chromatographieren erforderlich war. Das auf diesem Wege erhaltene Produkt 7 war diastereomerenrein, wie die Analyse des ${ }^{13} \mathrm{C}$ NMR-Spektrums und zusätzliche GC/MSUntersuchungen bewiesen. 


\subsection{Diskussion der spektroskopischen Daten von 7}

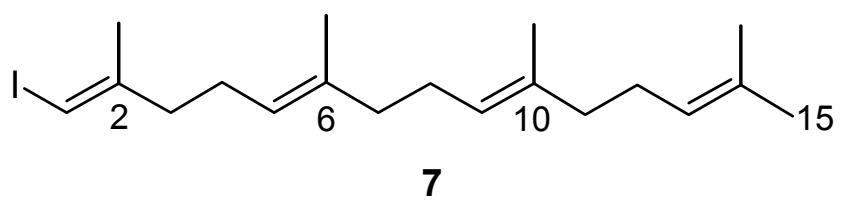

Ebenso wie bei Alkin 9 treten auch bei Verbindung 7 im UV-Spektrum zwischen $\lambda=190$ und $400 \mathrm{~nm}$ keine Absorptionsbanden auf, da die im Molekühl vorhandenen Doppelbindungen untereinander nicht in Konjugation stehen.

Das IR-Spektrum zeigt die Absorptionsbanden der olefinischen C-H-Streckschwingungen bei $\widetilde{v}=3054 \mathrm{~cm}^{-1}$ und die Absorptionbanden der aliphatischen C-H-Schwingungen im Bereich von 2966 bis $2854 \mathrm{~cm}^{-1}$. Einige für die Streckschwingungen der $\mathrm{C}=\mathrm{C}$-Doppelbindungen charakteristischen Absorptionen treten bei $\widetilde{v}=1714,1666,1618 \mathrm{~cm}^{-1}$ in Erscheinung.

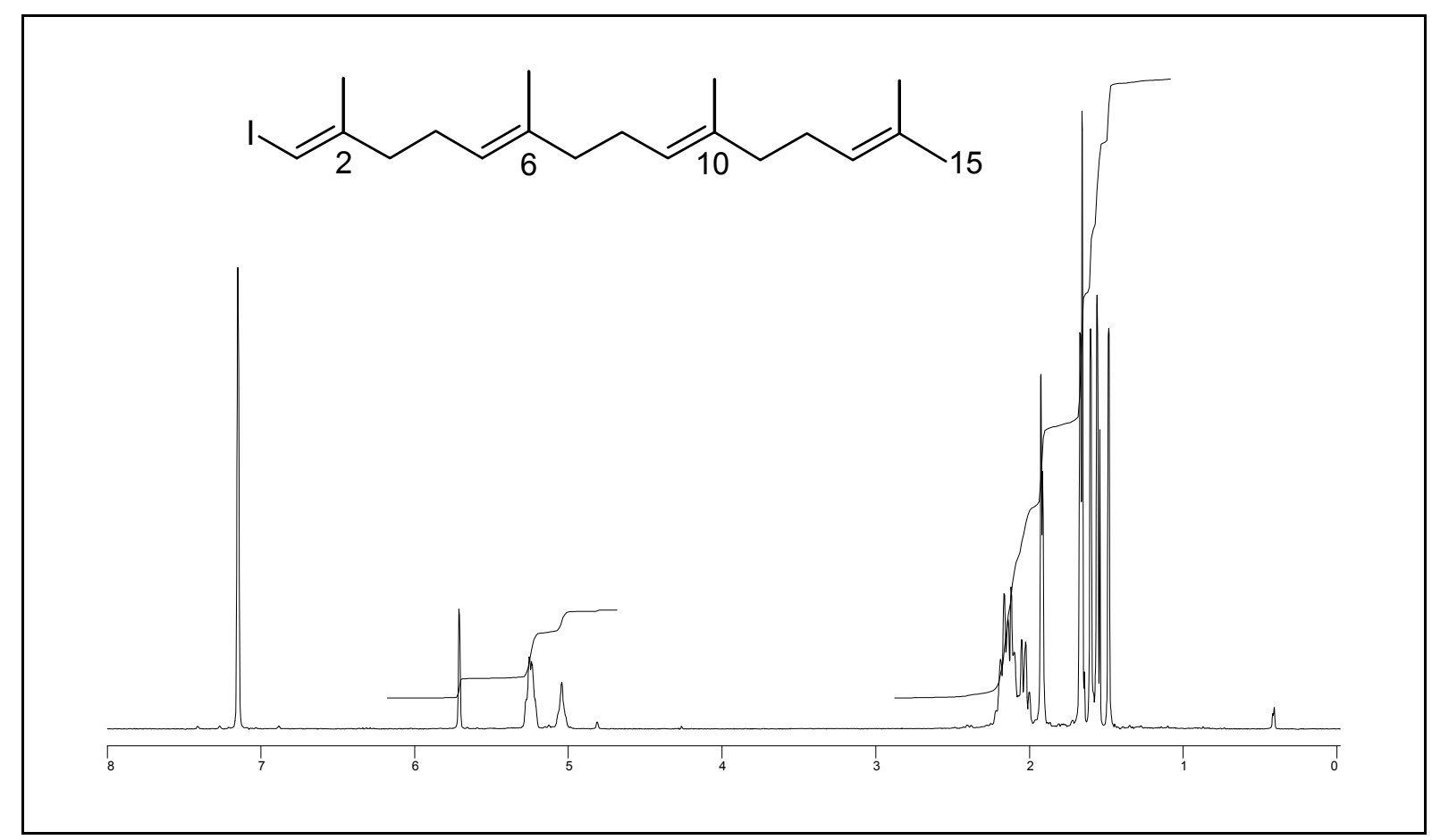

Abb. 49: ${ }^{1} \mathrm{H}$ NMR-Spektrum von Verbindung 7 (200 MHz, $\left.\mathrm{C}_{6} \mathrm{D}_{6}\right)$

Vergleicht man die ${ }^{1}$ H NMR-Spektren der Verbindungen 7 und 9, so läßt sich die erfolgreiche Umwandlung des terminalen Alkins in die entsprechende trisubstituierte Doppelbindung am Signal der neuen Methylgruppe bei $\delta=1.66 \mathrm{ppm}$ erkennen (Abbildung 49). Das 
entsprechende olefinische Proton 2-H resoniert, bedingt durch die Nachbarschaft des Iodatoms, bei $\delta=5.71 \mathrm{ppm}$.

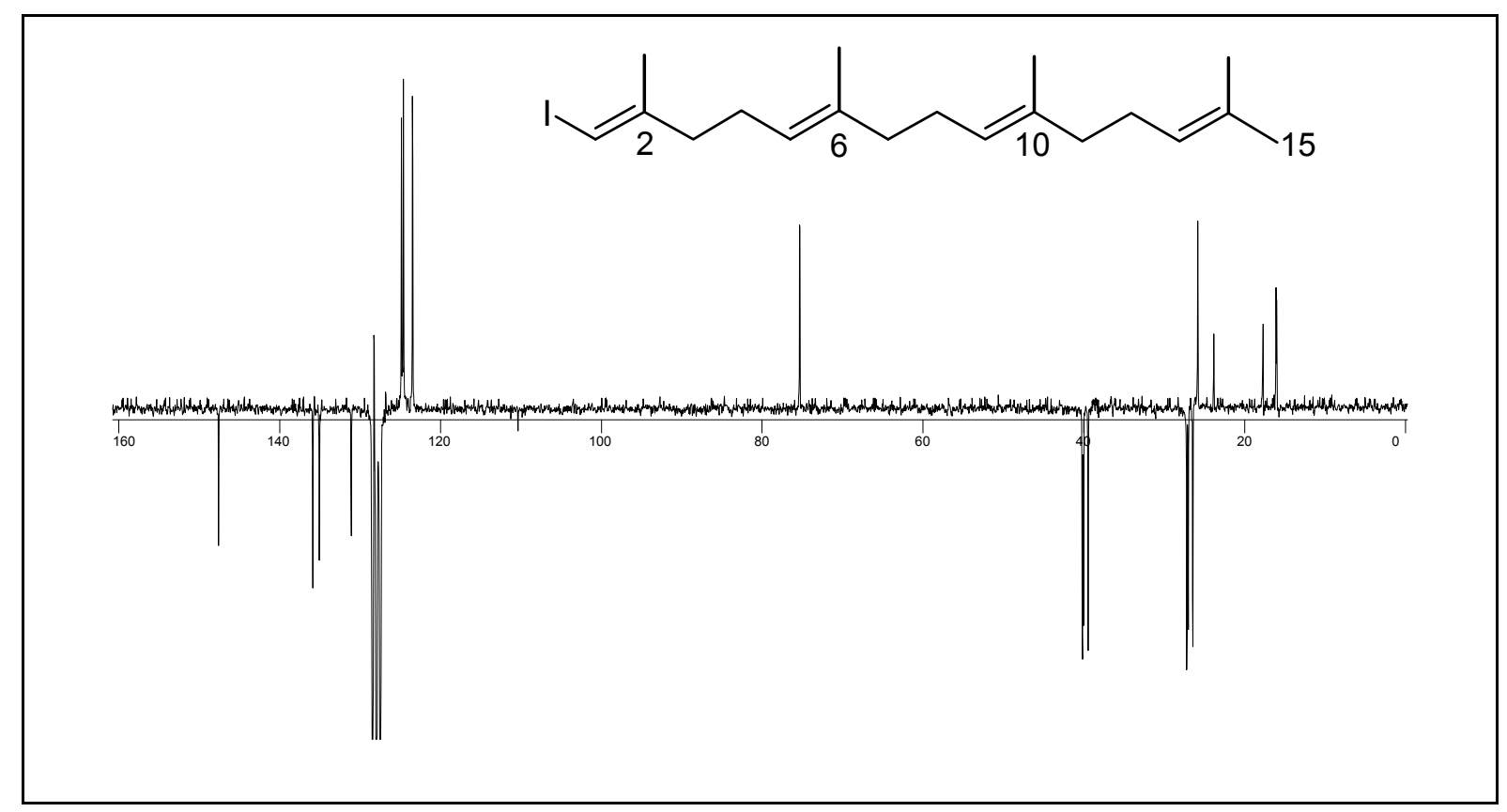

Abb. 50: ${ }^{13} \mathrm{C}$ APT NMR-Spektrum von Verbindung $7\left(50 \mathrm{MHz}, \mathrm{C}_{6} \mathrm{D}_{6}\right)$

Im ${ }^{13} \mathrm{C}$ NMR-Spektrum zeigt sich der Einfluß des Iodatoms am deutlichsten (Abbildung 50). Das mit dem Halogenatom direkt verbundene Atom C-1 erfährt die bei Iodatomen charakteristische Hochfeldverschiebung und resoniert bei $\delta=75.39 \mathrm{ppm}$. Der Einfluß auf das Atom C-2 führt erwartungsgemäß zu einer Tieffeldverschiebung ( $\beta$-Effekt) und läßt das entsprechende Signal bei $\delta=147.61 \mathrm{ppm}$ in Erscheinung treten. Von diesem $\beta$-Effekt erfährt auch die Methylgruppe 2- $\mathrm{CH}_{3}$, was dazu führt, daß ihr Signal bei $\delta=23.92 \mathrm{ppm}$ zu sehen ist. Das quartäre Kohlenstoffatom der Isopropylidengruppe präsentiert sein Signal bei $\delta=131.13$ ppm, während die endständigen olefinisch gebundenen Methylgruppen ebenfalls charakteristisch bei $\delta=17.80\left(14-\mathrm{CH}_{3}\right)$ und $25.90(\mathrm{C}-15)$ ppm resonieren.

Im Massenspektrum erscheint der Molpeak bei $\mathrm{m} / z=386$ mit geringer Intensität. Das durch den Verlust des Iodatoms entstehende Fragmention $\left[\mathrm{M}^{+}-\mathrm{I}\right]$ tritt bei $m / z=259$ in Erscheinung. Den Basispeak des Massenspektrums bildet das Fragmention $\left[\mathrm{C}_{5} \mathrm{H}_{9}{ }^{+}\right]$mit $\mathrm{m} / z=69$, was die zahlreichen Möglichkeiten zur Allylspaltung der isoprenoiden Verbindung 7 zum Ausdruck bringt. 


\subsection{Zusammenfassung}

Ausgehend von dem kommerziell erhältlichen, diastereomerenreinen $(E, E)$-Farnesylaceton $\mathbf{1 0}^{[97]}$ gelang die Synthese des diastereomerenreinen Vinyliodids 7 in wenigen Syntheseschritten. Nach einer Methode von Negishi und Mitarbeitern ${ }^{[99,100]}$ wurde $(E, E)$ Farnesylaceton (10) zunächst mit einer Ausbeute von $75 \%$ in das entsprechende terminale Alkin 9 übergeführt, das die Ausgangsbasis für die sich anschließende Carboaluminierung ${ }^{[96,101]}$ bildete. Diese ergab das angestrebte diastereomerenreine Vinyliodid 7 mit einer Ausbeute von $74 \%$. 


\section{Synthese von Methanophenazin (1)}

Ein Schlüsselschritt zur Herstellung von $(S)$-Methanophenazin $[(S)$-1] bildet die Verknüpfung des Alkyliodids $(R)-6$ mit dem Vinyliodid 7 beim Aufbau der Seitenkette $(S)$-3. Im weiteren Verlauf der Synthese soll dann durch Veretherung der Seitenkette (S)-3 mit dem Heteroaromaten 2 das Zielmolekül (S)-1 synthetisiert werden (Abbildung 15, S. 22).

Die Methoden zum stereoselektiven Aufbau trisubstituierter Doppelbindungen sind nicht sehr zahlreich. Eine Möglichkeit besteht in der übergangsmetallkatalysierten Kreuzkupplung eines Alkylmetallderivates mit einem Vinyliodid ${ }^{[14]}$. Sie wurde am Beispiel der Umsetzung von $(R)-6$ mit 7 zu $(S)$-76 untersucht. Liegt der $\mathrm{C}_{6}$-Baustein $(R)-6$ in Form seines GrignardReagenzes vor, dann sind katalytische Mengen eines Nickel-Komplexes notwendig, damit die $\mathrm{sp}^{2}$-sp ${ }^{3}$-Kupplung ablaufen kann. ${ }^{[116]}$ Transformiert man das Alkyliodid $(R)-6$ in die entsprechende Organozinkverbindung, dann hat sich Anwesenheit von $\operatorname{Pd}\left(\mathrm{PPh}_{3}\right)_{4}$ als notwendig erwiesen, um die gewünschte Verknüpfung $\mathrm{zu}$ erreichen. ${ }^{[117]}$ Alle genannten Reaktionen verlaufen unter Retention der Konfiguration am substituierten $\mathrm{sp}^{2}$-Zentrum.

\subsection{Palladium-katalysierte Kreuzkupplung von $(R)-6$ mit 7}

Die Lithiierung der Vinylposition von 7 durch Iod-Lithium-Austausch und anschließende Alkylierung mit $(R)-6$ nach einer Vorschrift von Mori und Mitarbeitern ${ }^{[104]}$ konnte nicht realisiert werden.

Bessere Ergebnisse wurden in Anlehnung an eine Vorschrift von Smith und Mitarbeitern ${ }^{[105]}$ durch Palladium-katalysierte Kreuzkupplung der beiden Seitenkettenbausteine 7 und $(R)-6$ erzielt. Dazu wird das Alkyliodid $(R)-6$ zunächst durch Behandeln mit trockenem Zinkchlorid und tert.-Butyllithium in das Zinkorganyl $(R)$-75 übergeführt.

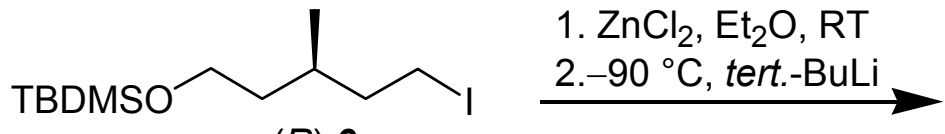

$(R)-6$

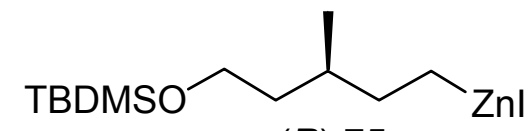

$(R)-75$

Dieses wird anschließend in Gegenwart von drei Molprozent $\mathrm{Pd}\left(\mathrm{PPh}_{3}\right)_{4}$ mit dem Vinyliodid 7 zum geschützten Alkohol (S)-76 gekuppelt. 
<smiles>CC=C(C)CCC=C(C)CCC=C(C)CCC=C(C)C</smiles>

7<smiles>CCOCC[C@H](C)CCC=C(C)CCC=C(C)CCC=C(C)CCC=C(C)C</smiles>

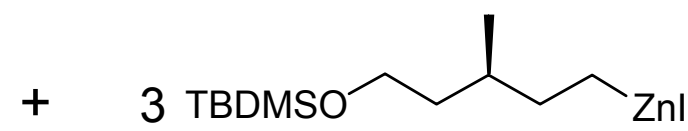

$(R)-75$

(S)-76

Die zu Beginn durchgeführten Versuche verliefen mit lediglich 40-45 \% Ausbeute an $(S)$-76. Als Nebenprodukt der Reaktion konnte das Homokupplungsprodukt 77 isoliert und anhand seines ${ }^{1} \mathrm{H}$ NMR- und Massenspektrums identifiziert werden.

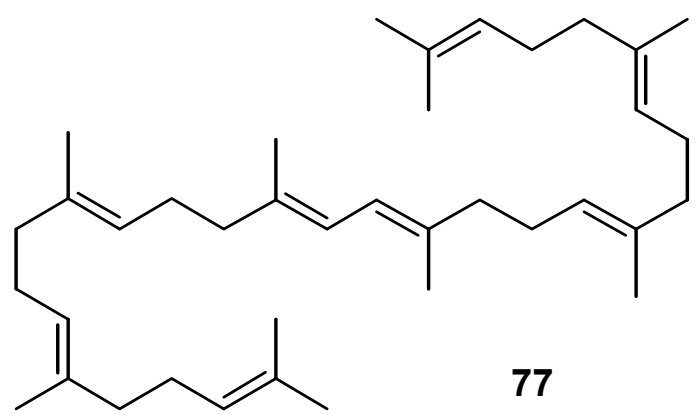

Eine Ausbeuteverbesserung wurde durch Erhöhung der Menge an $\mathrm{Pd}\left(\mathrm{PPh}_{3}\right)_{4}$ von drei auf neun Molprozent erzielt. Günstig wirkte sich auch aus, wenn die Vereinigung des ZinkOrganyls $(R)$-75 mit der ungesättigten Verbindung 7 nicht tropfenweise, sondern schnell und ohne Unterbrechung durchgeführt wurde. Vermutlich wird durch den so erzeugten Überschuß an Metallorganyl (R)-75 die Homokupplungsreaktion von zwei Molekülen 7 etwas zurückgedrängt. Die Ausbeute der Reaktion konnte im Verlauf mehrerer Versuche auf $65 \%$ gesteigert werden. Neben $\mathrm{Pd}\left(\mathrm{PPh}_{3}\right)_{4}$ kamen auch die Katalysatoren $\mathrm{PdCl}_{2}(\mathrm{dppf})$ und $\mathrm{PdCl}_{2}\left(\mathrm{PPh}_{3}\right)_{4}$ zum Einsatz. Mit Ausbeuten zwischen 30 und $40 \%$ ließ sich aber so keine Verbesserung erreichen. 
Die übergangsmetallkatalysierte C-C-Kupplung verläuft wie erwartet unter vollständiger Retention der Konfiguration des Vinyliodids 7. Das wird durch die Analyse des ${ }^{13} \mathrm{C}$ NMRSpektrums und durch GC-MS Untersuchungen des Kupplungsprodukts bestätigt.

Die mechanistischen Vorstellungen ${ }^{[106]}$ über den Ablauf Palladium-katalysierter Kreuzkupplungen gehen davon aus, daß sich im ersten Schritt $\operatorname{Pd}(0)$ durch oxidative Addition in die $\mathrm{C}\left(\mathrm{sp}^{2}\right)-\mathrm{I}$ Bindung des Vinylhalogenids einschiebt, wobei $\operatorname{Pd}(0) \mathrm{zu} \operatorname{Pd}(+\mathrm{II})$ oxidiert wird. Im nächsten Schritt kommt es dann zu einer Ummetallierung der Alkyl-Zink-Verbindung, wobei ein Alkylrest auf das Palladium übertragen wird und so eine Alkyl-Palladium-Verbindung entsteht. Die abschließende reduktive Eliminierung verläuft schneller als die mit ihr konkurrierende $\beta$-Hydrid-Eliminierung. Dabei entsteht unter Retention der Konfiguration des $\mathrm{sp}^{2}$-C-Atoms das $\mathrm{sp}^{2}$-sp ${ }^{3}$-Kupplungsprodukt und die niedervalente katalytisch aktive $\operatorname{Pd}(0)$ Spezies wird regeneriert.

\subsection{Desilylieren der Seitenkette $(S)-76$}

Die flouridinduzierte Abspaltung der Schutzgruppe gelingt mühelos und nahezu quantitativ, durch kurzzeitiges Erwärmen des Kupplungsproduktes (S)-76 mit Tetrabutylammoniumfluorid in Tetrahydrofuran.

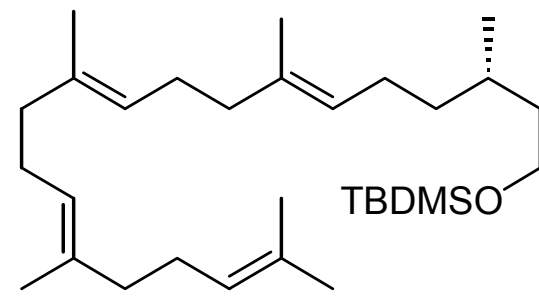

(S)-76
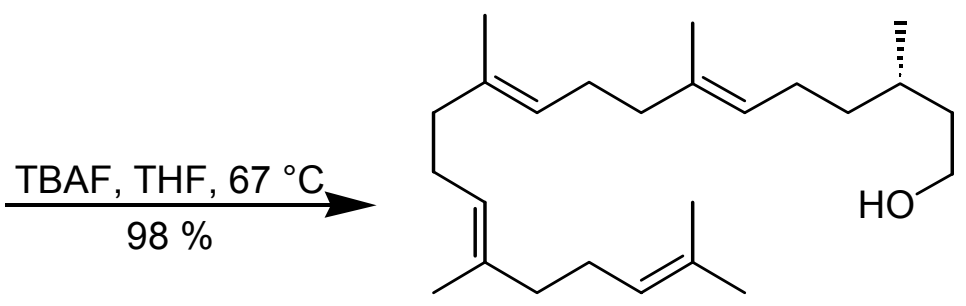

(S)-78

Im Verlauf der Synthese von $(S)$-78 hat sich herausgestellt, daß die Reinigung des geschützten Kupplungsprodukts $(S)-76$ nicht in jedem Fall notwendig ist. Am einfachsten ist es, das rohe Kupplungsprodukt $(S)$-76 ohne weitere Aufreinigung in den entsprechenden Alkohol (S)-78 überzuführen. Die Isolierung des chiralen Alkohols $(S)$-78 gelingt dann durch einfache Chromatographie an Kieselgel. Die Verwendung des rohen $(S)$-76 mindert nicht die Ausbeute an (S)-78. 


\subsection{Bestimmung der Enantiomerenreinheit von $(S)-78$}

Wie bereits in Kapitel 8.3 berichtet, ist zur Bestimmung des Enantiomerenüberschusses von primären Alkoholen die in beiden Enantiomeren verfügbare $\alpha$-Methoxy- $\alpha$ (trifluormethyl)phenylessigsäure (62) das Reagenz der Wahl.<smiles>CC(C)=CCCC(C)=CCCC(C)=CCCC(C)CCO</smiles>

rac-78
(S)- $\alpha$-Methoxy- $\alpha$-trifluormethylphenylessigsäure [(S)-62)], DCC, DMAP, $\mathrm{CH}_{2} \mathrm{Cl}_{2}$

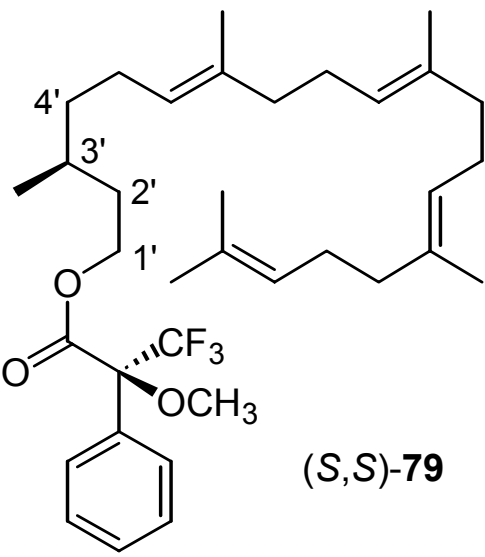
$70 \%$

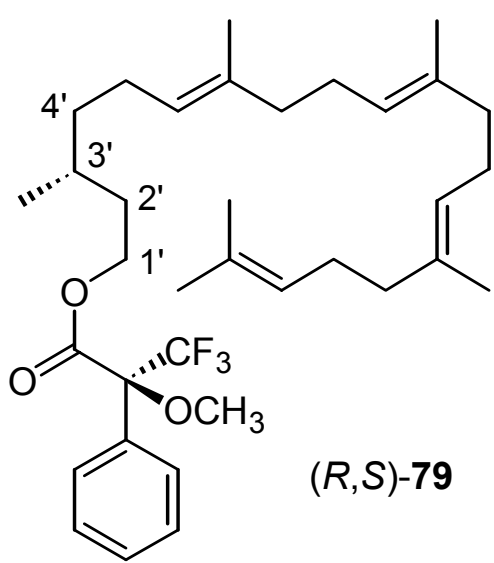

Nach der Veresterung von rac-78 mit $S(-)$ - $\alpha$-Methoxy- $\alpha$-(trifluormethyl)phenylessigsäure $\left[(S)\right.$-62] und Analyse der ${ }^{19} \mathrm{~F}$-, ${ }^{1} \mathrm{H}$ - und ${ }^{13} \mathrm{C}$ NMR-Spektren der diasterereomeren Produkte $(R, S)-79$ und $(S, S)-79$ wies nur das ${ }^{13} \mathrm{C}$ NMR-Spektrum Signale auf, die zur Bestimmung der optischen Reinheit des Alkohols (S)-78 geeignet waren. (Abbildung 51). Es sind die Signale der Kohlenstoffatome C-1', C-2', C-4' und 3'- $\mathrm{CH}_{3}$ der beiden Diastereomere $(R, S)-79$ und $(S, S)$-79, die unterschiedliche chemische Verschiebungen zeigen und so zur Enantiomerenanalytik herangezogen wurden. 


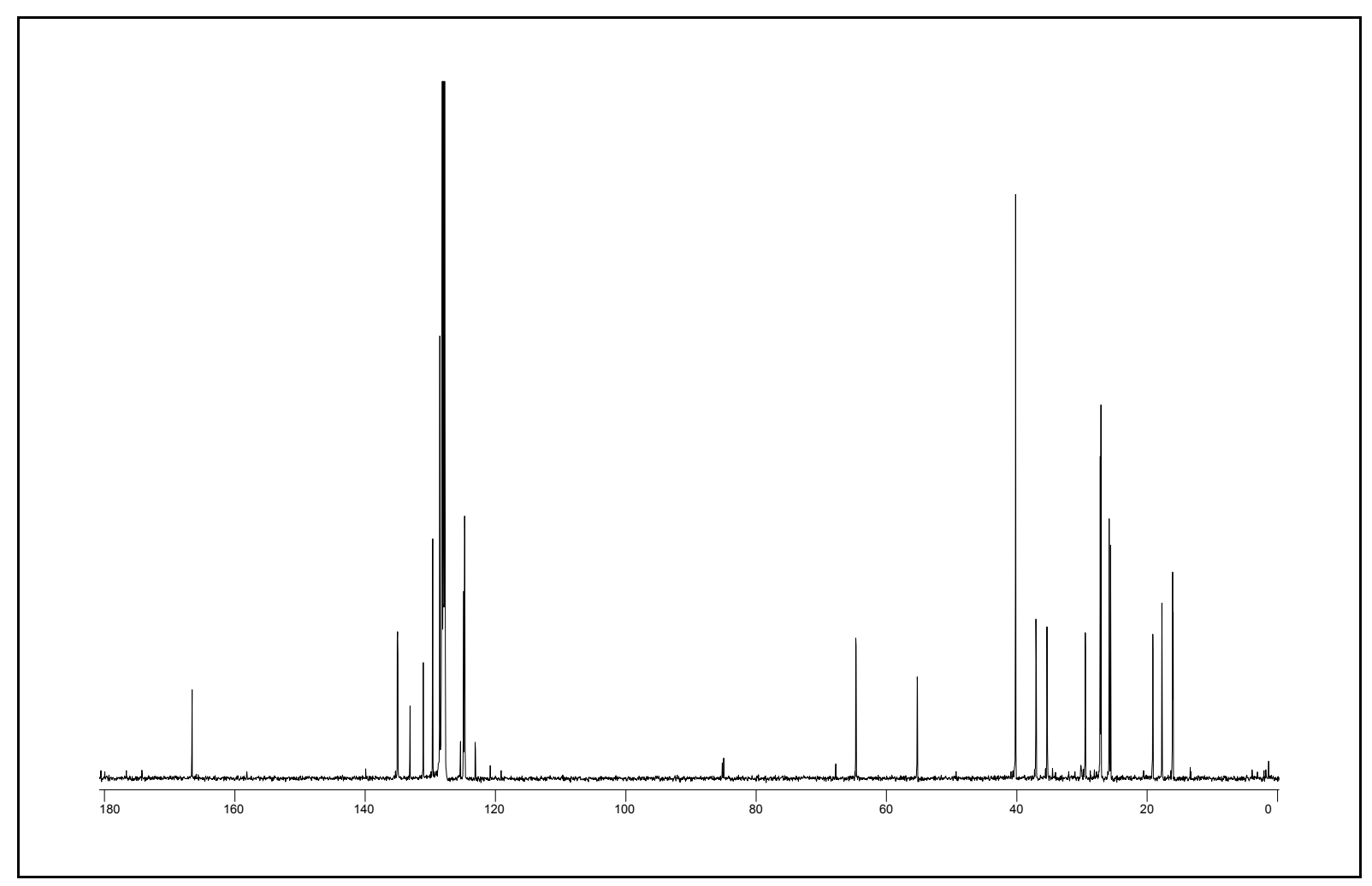

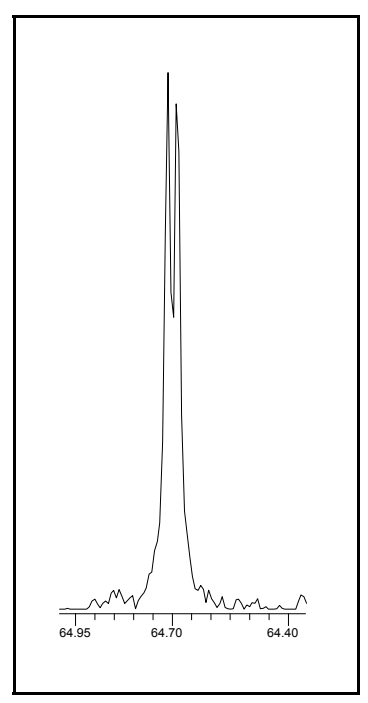

C-1'

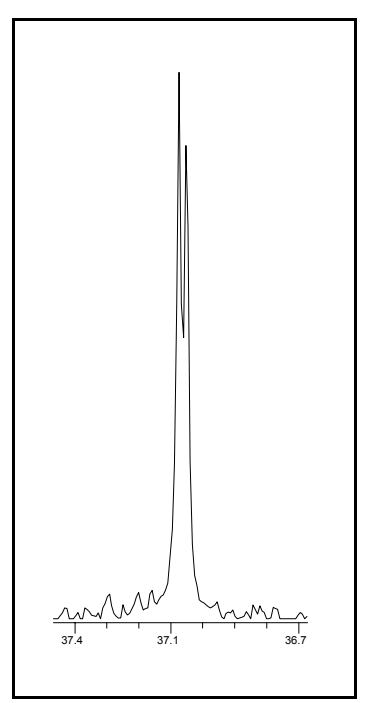

C-2'

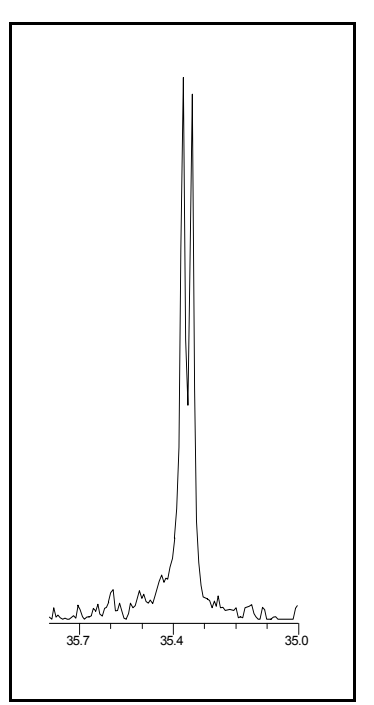

C-4'

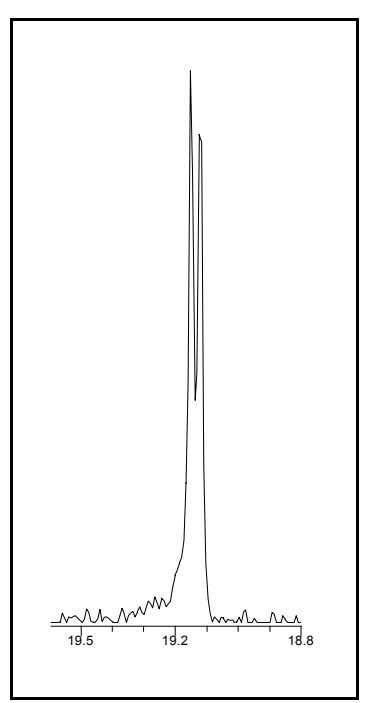

3'- $\mathrm{CH}_{3}$

Abb. 51: ${ }^{13} \mathrm{C}$ NMR-Spektrum der Mosher-Ester $(R, S)-79$ und $(S, S)-79$ mit Dehnungen der Signale, die zur Bestimmung des Diastereomerenverhältnis herangezogen wurden $\left(125 \mathrm{MHz}, \mathrm{C}_{6} \mathrm{D}_{6}\right.$, Diastereomerenverhältis = $1: 1$ ) 
Die Reaktion der chiralen Seitenkette $(S)$-78 mit der enantiomerenreinen Carbonsäure $(S)$-62 ergibt den Ester $(S, S)-79$ mit $83 \%$ Ausbeute. Die Analyse des ${ }^{13} \mathrm{C}$ NMR-Spektrums läßt nur den Signalsatz des (S,S)-79 Diastereomers erkennen (Abbildungen 52 und 53). Der Alkohol $(S)-78$ liegt demnach mit einem Enantiomerenüberschuß von wenigstens $>95 \%$ vor. ${ }^{[107]}$ Die Behandlung des nahezu enantiomerenreinen (ee $>95 \%$ ) Alkyliodids $(R)-6$ mit $\mathrm{ZnCl}_{2}$ und tert. Butyllithium, sowie die anschließende Palladium-katalysierte Kupplung mit 7 führt also nicht zur Racemisierung am stereogenen Zentrum des $\mathrm{C}_{6}$-Bausteins $(R)$-6.
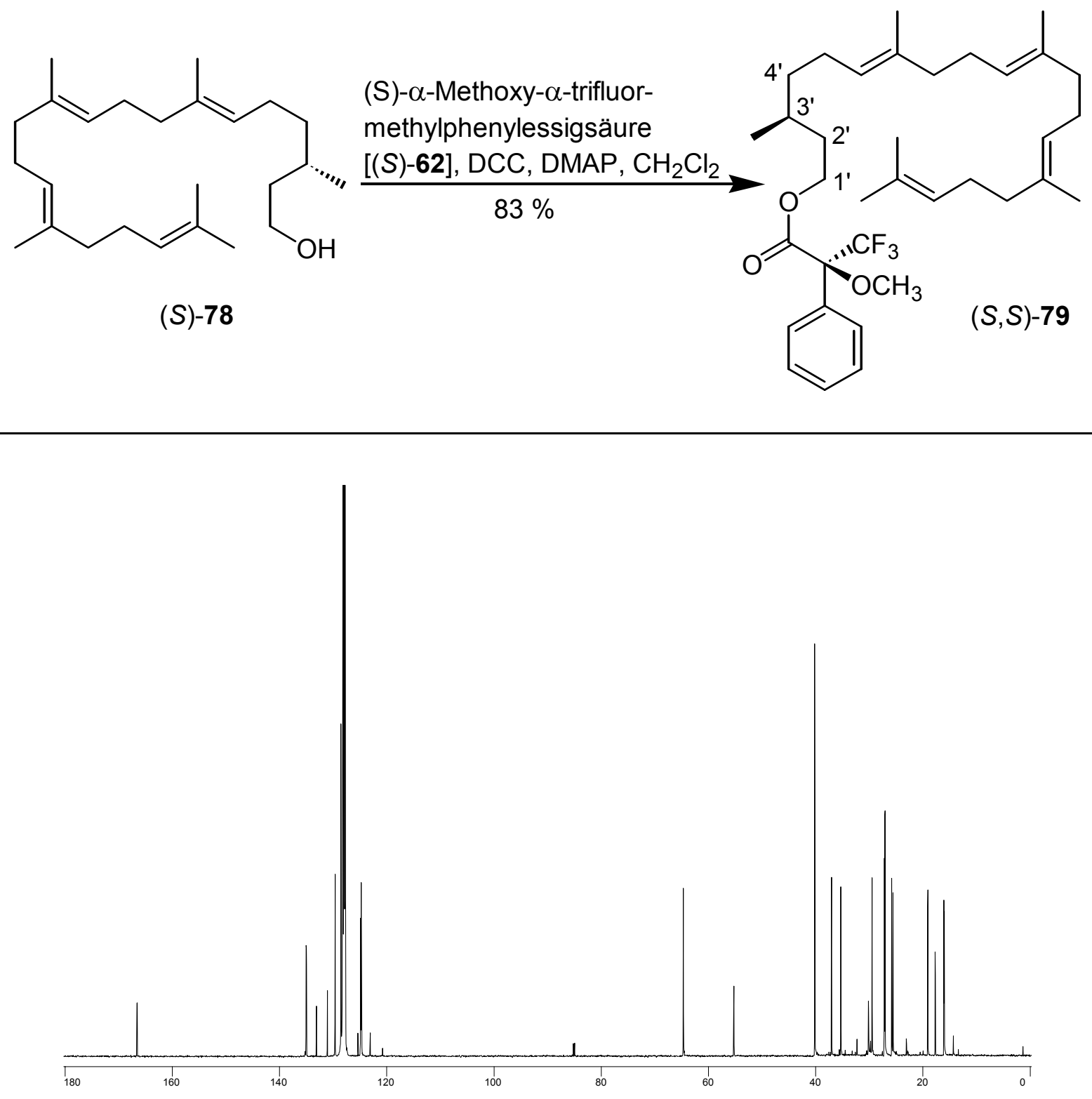

Abb. 52: ${ }^{13} \mathrm{C}$ NMR-Spektrum des Mosher-Esters (S,S)-79 (125 MHz, $\left.\mathrm{C}_{6} \mathrm{D}_{6}\right)$ 


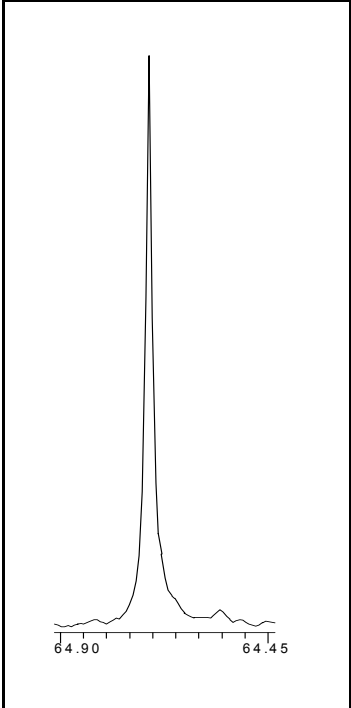

C-1

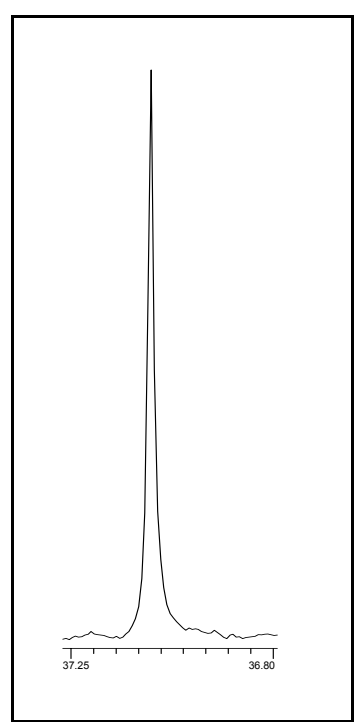

C-2'

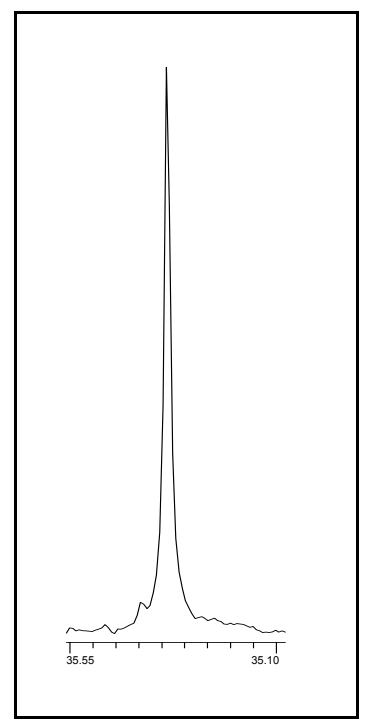

C-4'

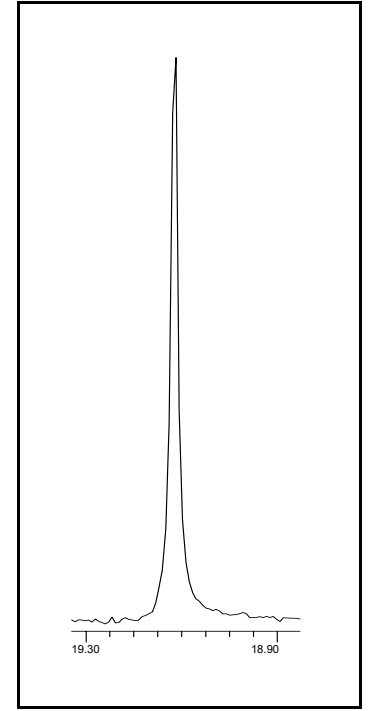

$3^{\prime}-\mathrm{CH}_{3}$

Abb. 53: Dehnungen der Signale, die zur Bestimmung des Diastereomerenverhältnis herangezogen wurden

\subsection{Diskussion der spektroskopischen Daten von $(S)-78$}<smiles>[2H]C(C)=CCC/C(C)=C/CC/C(C)=C/CC/C(C)=C/CC[C@H](C)CCO</smiles>

$(S)-78$

Die Verbindung $(S)$-78 ist ihrer Natur nach ein farbloses Öl, das im UV-Spektrum zwischen $\lambda=190$ und $400 \mathrm{~nm}$ erwartungsgemäß keine Absorptionsbanden zeigt. Der spezifische Drehwert beträgt $[\alpha]_{D}^{20}=-2.6^{\circ}$. Im IR-Spektrum ist die breite Bande bei $\widetilde{v}=3331 \mathrm{~cm}^{-1}$ ein Beleg für die freie Hydroxylgruppe im Molekül. Die C-O-Streckschwingung absorbiert bei $\widetilde{v}=1057 \mathrm{~cm}^{-1}$. Die aliphatischen C-H-Streckschwingungen der zahlreichen Methylen- und Methylgruppen absorbieren bei $\widetilde{v}=2960,2924$ und $2854 \mathrm{~cm}^{-1}$. Die Bande bei $\widetilde{v}=1666$ $\mathrm{cm}^{-1}$ wird der $\mathrm{C}=\mathrm{C}$-Streckschwingung einer trisubstituierten Doppelbindung zugeordnet. 


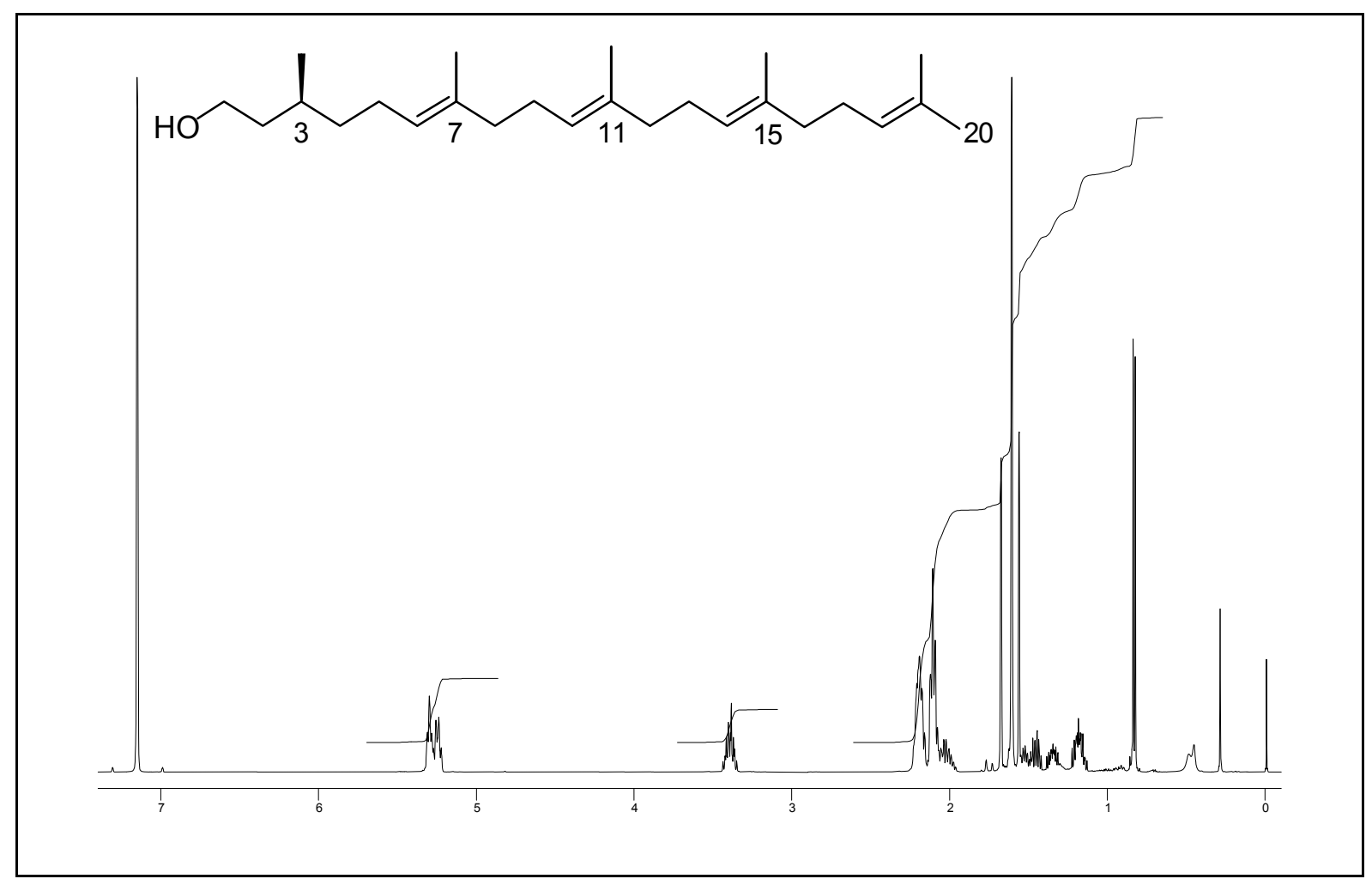

Abb. 54: ${ }^{1} \mathrm{H}$ NMR-Spektrum von Verbindung $(S)-78\left(500 \mathrm{MHz}, \mathrm{C}_{6} \mathrm{D}_{6}\right)$

Das ${ }^{1} \mathrm{H}$ NMR-Spektrum (Abbildung 54) präsentiert die Methylgruppe am stereogenen Zentrum bei $\delta=0.83 \mathrm{ppm}$ als ein durch die ${ }^{3} J$-Kopplung zum benachbarten Methinproton zum Dublett aufgespaltenes Signal. Vier starke Singuletts der Methylgruppen 7- $\mathrm{CH}_{3}, 11-\mathrm{CH}_{3}$, $15-\mathrm{CH}_{3}$ und $19-\mathrm{CH}_{3}$ dominieren das Spektrum im Bereich von $\delta=1.56$ bis $1.61 \mathrm{ppm}$. Sie werden eingerahmt durch die Signale der $20-\mathrm{H}_{3}$ Protonen bei $\delta=1.67 \mathrm{ppm}$, die als Dublett erscheinen, sowie dem mit Deuterium austauschbaren Hydroxylproton bei $\delta=1.54 \mathrm{ppm}$. Die Methylenprotonen 2- $\mathrm{H}_{2}, 3-\mathrm{H}$ und $4-\mathrm{H}_{2}$ resonieren im Bereich von $\delta=1.10$ bis $1.53 \mathrm{ppm}$, während die allylischen Methylengruppen in einem schwach aufgelösten Resonanzsignal den Bereich von $\delta=1.94$ bis 2.25 ppm des Spektrums belegen. Durch die unmittelbare Nähe des Sauerstoffatoms zu tiefem Feld verschoben, resonieren die diastereotopen 1- $\mathrm{H}_{2}$ Protonen bei $\delta=3.39$ und $3.41 \mathrm{ppm}$. Bei tiefstem Feld treten erwartungsgemäß die vier olefinischen Protonen 6-H, 10-H, 14-H und 18-H als Multiplett von $\delta=5.21$ bis 5.35 ppm in Erscheinung. 


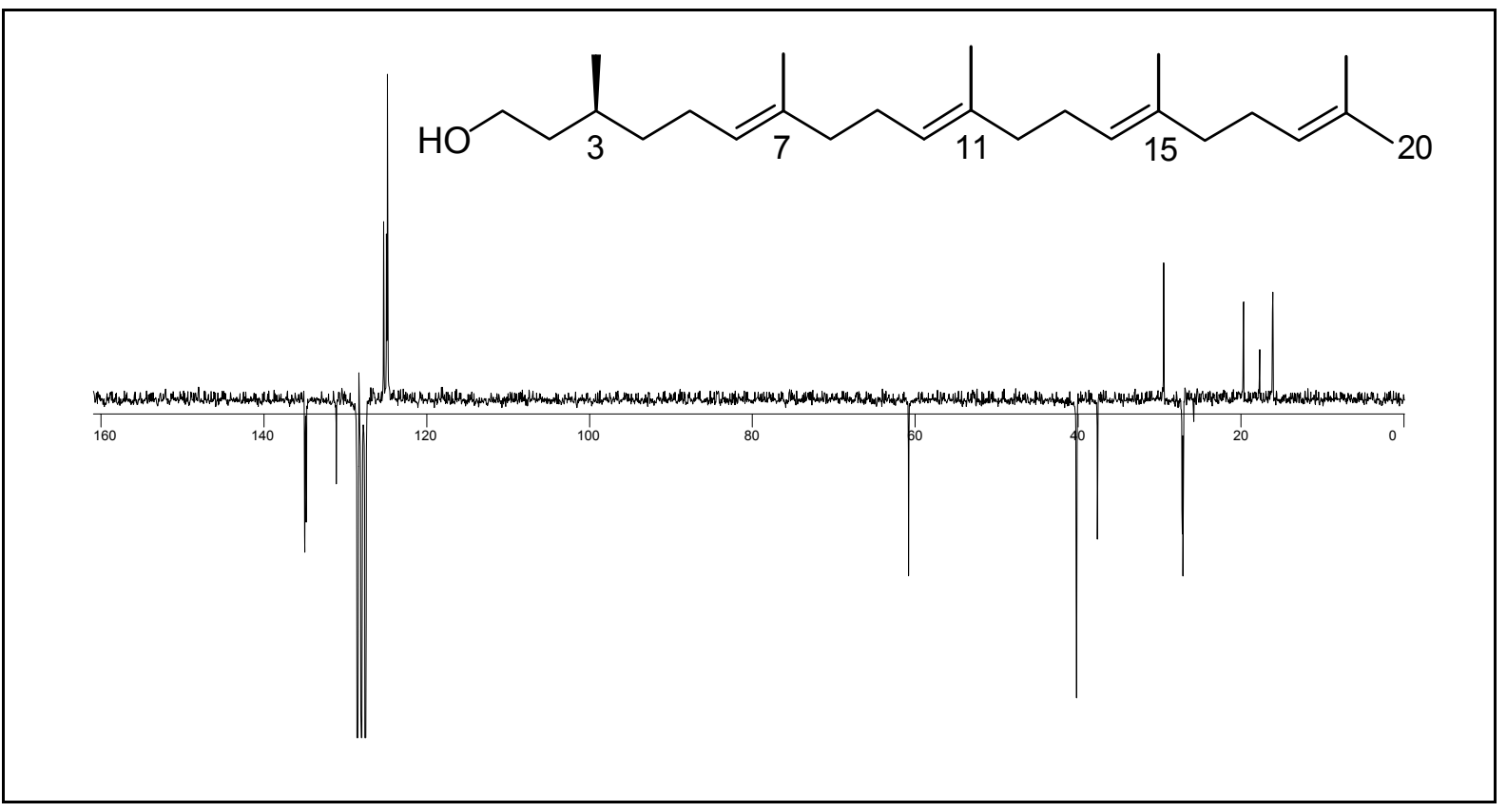

Abb. 55: ${ }^{13} \mathrm{C}$ APT NMR-Spektrum von Verbindung (S)-78 (50 MHz, $\left.\mathrm{C}_{6} \mathrm{D}_{6}\right)$

Von der gelungenen Kreuzkupplung der beiden Fragmente $(R)-6$ mit 7 sowie der anschließenden Desilylierung zeugen im ${ }^{13} \mathrm{C}$ NMR-Spektrum (Abbildung 55) u. a. die Resonanzen der tertiären C-Atome des olefinischen Teils von $(S)-78$ bei $\delta=124.80$ (C-10, C-14), 124.93 (C6) und 125.27 (C-18) ppm sowie das stereogene Kohlenstoffatom C-3 des aliphatischen Teils von $(S)-78$ bei $\delta=29.49$ ppm. Die dazugehörigen quartären Kohlenstoffatome C-7, C-11 und C-15 präsentieren ihre Signale im Bereich von bei $\delta=134.78$ bis 134.99 ppm. Eine Ausnahme bildet das quartäre Atom C-19. Es resoniert bei $\delta=131.10 \mathrm{ppm}$. Zusammen mit den daran gebundenen Methylgruppen $1^{\prime}-\mathrm{CH}_{3}$ und $20-\mathrm{H}_{3}$ bei $\delta=17.75$ und 25.86 ppm markieren sie eine Isopropylidengruppe und unterscheiden sich damit charakteristisch von den entsprechenden Kohlenstoffatomen inmitten der Kette. Neben den bereits erwähnten Signalen der beiden terminalen Methylgruppen finden sich Resonanzen der Atome 7- $\mathrm{CH}_{3}, 11$ $\mathrm{CH}_{3}$ und 15- $\mathrm{CH}_{3}$ im Bereich von $\delta=16.09$ bis 16.15 ppm. Das Signal der Methylgruppe am Stereozentrum tritt bei $\delta=19.71 \mathrm{ppm}$ in Erscheinung. Bedingt durch die Bindung zum Sauerstoffatom wird das Atom C-1 entschirmt und sein Resonanzsignal erfährt eine Tieffeldverschiebung zu $\delta=60.83 \mathrm{ppm}$. 


\subsection{Darstellung von (S)-Methanophenazin [(S)-1]}

Nachdem nun 2 und $(S)$-78 vorlagen, war zum erfolgreichen Abschluß der Synthese von $(S)$ Methanophenazin [(S)-1] nur noch die Verknüpfung dieser beiden Bausteine durch eine Veretherung erforderlich. Wie in Kapitel 7.1 dargelegt, ergaben Modellversuche mit 2Hydroxyphenazin (2) und Citronellol ( $r a c-47)$ gute Ausbeuten an Ether, wenn rac-47 in Form seines Mesylats mit 2 in Gegenwart von Kaliumhydroxid in Tetrahydrofuran unter Phasentransferkatalyse zur Reaktion gebracht wurden. Unter den gleichen Bedingungen entsteht Methanophenazin [(S)-1] aus dem Mesylat von (S)-78 und 2-Hydroxyphenazin (2) in $80 \%$ Ausbeute. Die Bildung eines nicht identifizierten Nebenproduktes kann durch Steigerung der Reaktionstemperatur, Verkürzung der Reaktionszeit und Verwendung von Toluol als Lösungsmittel vermindert werden. Der Einsatz eines Überschusses an 2Hydroxyphenazin (2) (1.2 Äquivalente) ist notwendig, um den vollständigen Umsatz des Mesylats von (S)-78 zu gewährleisten. Dies ist notwendig, da sich nicht umgesetztes Mesylat (S)-3 und das Reaktionsprodukt $(S)$-1 bei der chromatographischen Reinigung nur schwer voneinander trennen lassen. Unter den optimierten Reaktionsbedingungen liegt die Ausbeute an $(S)-1$ bei $90 \%$.

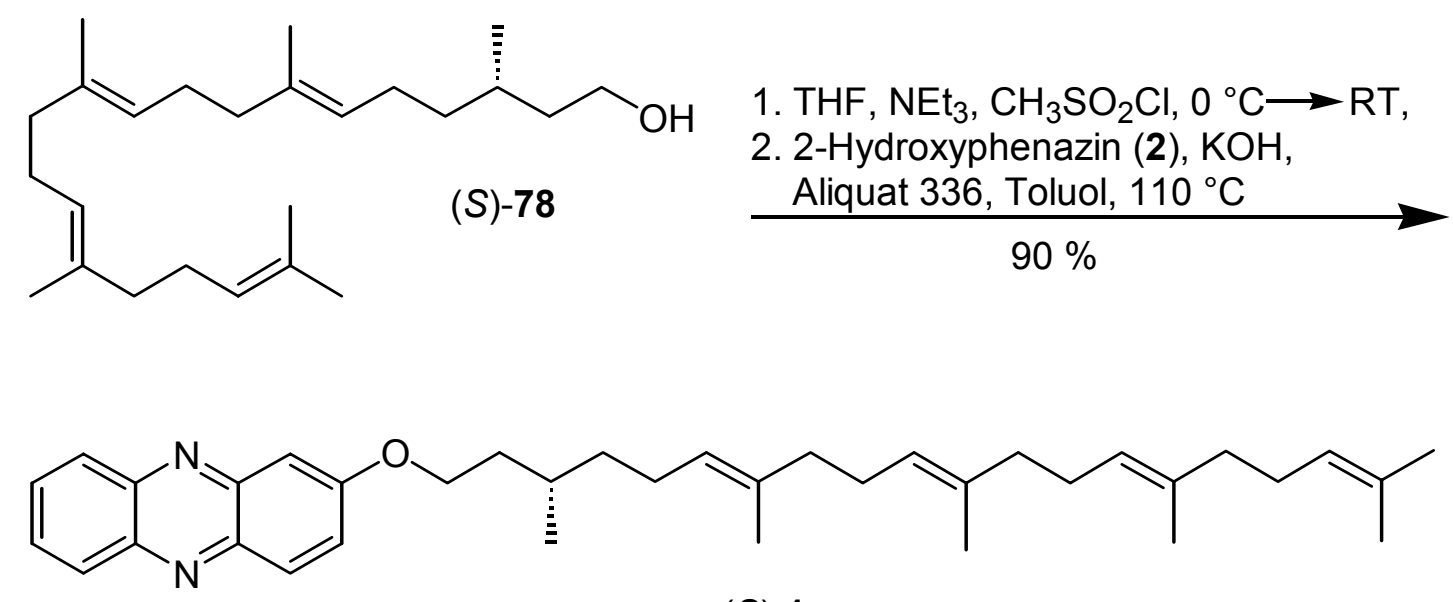

$(S)-1$

Damit war die Synthese von (S)-Methanophenazin [(S)-1] abgeschlossen. Sie verlief ausgehend von $(E, E)$-Farnesylaceton (10) in 6 Schritten und lieferte die Verbindung $(S)-\mathbf{1}$ in enantiomerenreiner Form mit einer Gesamtausbeute von $32 \%$. 


\subsection{Darstellung von racemischen Methanophenazin $r a c-1$}

Die Synthese des racemischen Naturstoffs rac-1 geht von dem in Kapitel 8.1 beschriebenen Alkyliodid rac-6 aus. Seine Verknüpfung mit dem ungesättigten $\mathrm{C}_{19}$-Baustein 7 verläuft nach derselben Methode, nach der auch $(S)$-1 synthetisiert wird, also durch eine Palladiumkatalysierte Kreuzkupplung der beiden Bausteine rac-6 und $7 \mathrm{zu}$ rac-76. Nach Entschützen und Aktivieren von rac-76 wird die abschließende Veretherung mit 2-Hydroxyphenazin (2) zu rac-1 in der oben beschriebenen Art und Weise durchgeführt.<smiles>CC=C(C)CCC=C(C)CCC=C(C)CCC=C(C)C</smiles>

7
1. rac-6, $\mathrm{ZnCl}_{2}, \mathrm{Et}_{2} \mathrm{O}, \mathrm{RT}$, dann tert.-BuLi,-90 ${ }^{\circ} \mathrm{C} \longrightarrow \mathrm{RT}$, Transfer $\frac{\text { zu 7, kat. } \mathrm{Pd}\left[\mathrm{P}(\mathrm{Ph})_{3}\right]_{4}, \mathrm{RT}}{65 \%}$<smiles>CC(C)=CCC/C(C)=C\CC/C(C)=C/CC/C(C)=C/CCC(C)CCOS(C)(=O)=O</smiles>

rac-76

1. TBAF, THF, RT

2. $\mathrm{THF}, \mathrm{NEt}_{3}, \mathrm{CH}_{3} \mathrm{SO}_{2} \mathrm{Cl}$

3. 2-Hydroxyphenazin (2) $\mathrm{KOH}$, Aliquat 336, Toluol $90 \%$<smiles>CC(C)=CCCC(C)=CCCC(C)=CCCC(C)=CCCC(C)CCOc1ccc2nc3ccccc3nc2c1</smiles>

rac-1

\subsection{Diskussion der spektroskopischen Daten von $(S)$-Methanophenazin $[(S)-1)]$}<smiles>CC(C)=CCC/C(C)=C/CC/C(C)=C/CC/C(C)=C/CC[C@H](C)CCOc1ccc2nc3ccccc3nc2c1</smiles>

6

S-1

$(S)$-Methanophenazin $[(S)-1]$ ist ein gelbes Öl, das im UV-Spektrum Absorptionsmaxima bei $\lambda=256$, 354, und $388 \mathrm{~nm}$ aufweist. Das Stereozentrum an C-3' ist verantwortlich für die Drehung linear polarisierten Lichtes. Der höchste Drehwert $[\alpha]_{546}^{20}=-4.6^{\circ}$ wurde am Polarimeter bei Untersuchungen mit einer Wellenlänge von $\lambda=546 \mathrm{~nm}$ gemessen. Im IR Spektrum finden sich die Signale der aromatischen bzw. olefinischen C-H-Valenz- 
schwingungen bei $\widetilde{v}=3059 \mathrm{~cm}^{-1}$. Die entsprechenden Schwingungsbanden der Methylenund Methylgruppen präsentieren sich im Bereich von $\widetilde{v}=2954$ bis $2853 \mathrm{~cm}^{-1}$. Absorptionsbanden bei $\widetilde{v}=1632$ und $1605 \mathrm{~cm}^{-1}$ deuten auf olefinische-, solche bei $\widetilde{v}=1560$ und $1518 \mathrm{~cm}^{-1}$ auf aromatische $\mathrm{C}=\mathrm{C}$-Valenzschwingungen hin. Mittelstarke Absorptionsbanden von $\mathrm{CH}_{2}$ - und $\mathrm{CH}_{3}$-Deformationsschwingungen treten im Bereich von $\widetilde{v}=1483$ bis $1360 \mathrm{~cm}^{-1}$ in Erscheinung. Eine Absorption bei $\widetilde{v}=1196 \mathrm{~cm}^{-1}$ wird einer C-OValenzschwingung der Ethergruppe zugeordnet.

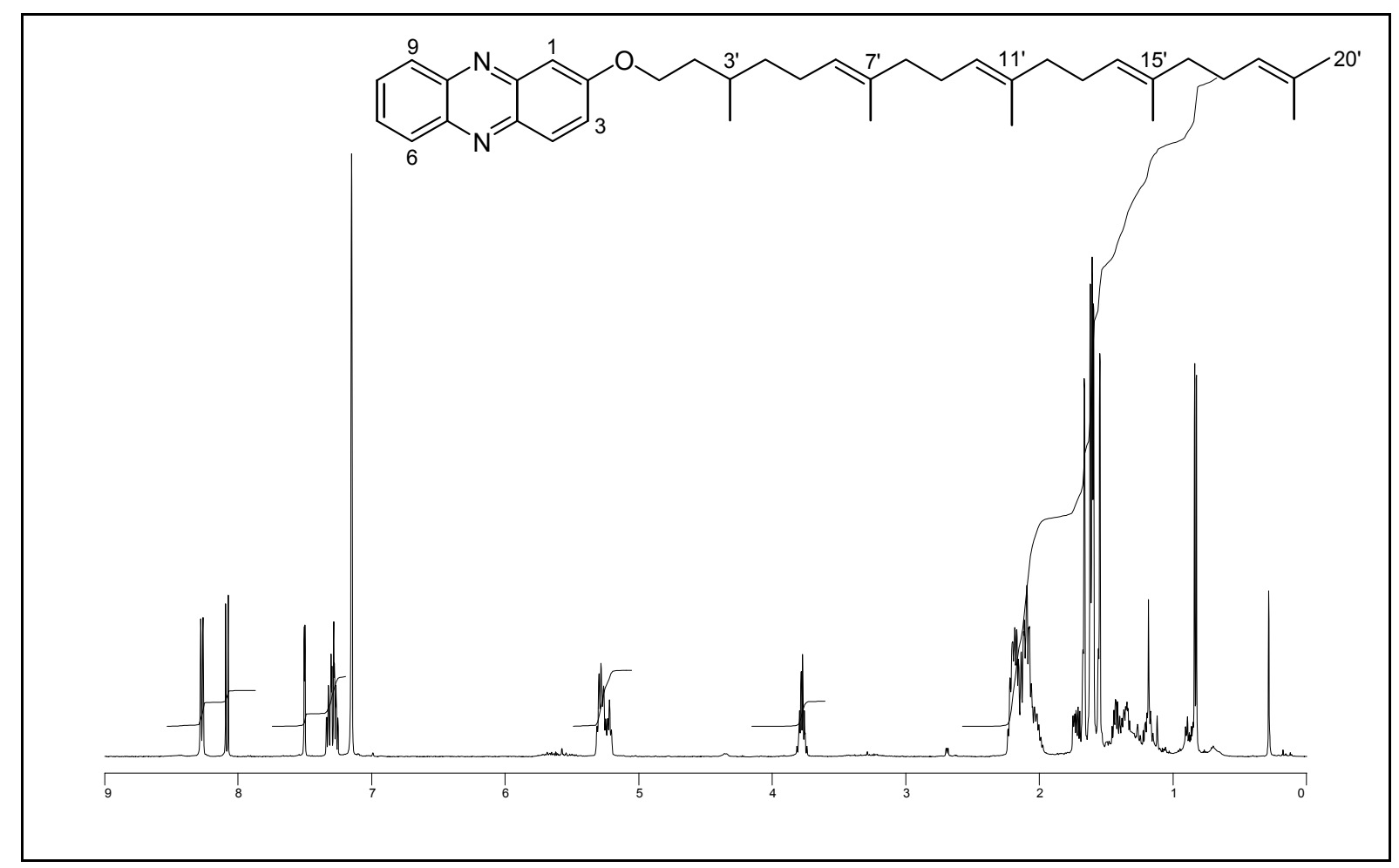

Abb. 56: ${ }^{1} \mathrm{H}$ NMR-Spektrum von natürlichem Methanophenazin (1) $\left(500 \mathrm{MHz}, \mathrm{C}_{6} \mathrm{D}_{6}\right)$

Im ${ }^{1} \mathrm{H}$ NMR-Spektrum zählt man die Resonanzsignale von sieben aromatischen, vier olefinischen und 39 aliphatischen Protonen (Abbildungen 56 und 57). Der aliphatische Bereich wird dominiert von den Resonanzen der fünf an Doppelbindungen gebundenen olefinischen Methylgruppen bei $\delta=1.56 \mathrm{ppm}\left(19^{\prime}-\mathrm{CH}_{3}\right), \delta=1.61$ bis $1.64 \mathrm{ppm}\left(7^{\prime}-\mathrm{CH}_{3}, 11^{\prime}\right.$ $\left.\mathrm{CH}_{3}, 15^{\prime}-\mathrm{CH}_{3}\right)$, sowie bei $\delta=1.68 \mathrm{ppm}\left(20^{\prime}-\mathrm{H}_{3}\right)$. Sie überlagern das Signal des 3 '- $\mathrm{H}$ bei $\delta=$ 1.60 bis 1.65 ppm. Das Signal der Methylgruppe 3'- $\mathrm{CH}_{3}$ am stereogenen Zentrum erscheint bei $\delta=0.83 \mathrm{ppm}$ und wird durch die ${ }^{3} J$-Kopplung mit dem benachbarten 3 '-H Proton charakteristisch zum Dublett aufgespalten. Die diastereotopen Protonen 2'- $\mathrm{H}_{2}$, und 4'- $\mathrm{H}_{2}$ treten als Multiplett zwischen $\delta=1.14$ und 1.44 ppm in Erscheinung. Alle allylischen 
Methylenprotonen resonieren als wenig aufgelöstes Multiplett bei $\delta=1.97$ bis $2.27 \mathrm{ppm}$. Der negative induktive Effekt des Sauerstoffatoms entschirmt die 1 ' $-\mathrm{H}_{2}$ Protonen und das Spektrum zeigt ihre Signale bei $\delta=3.80 \mathrm{ppm}$.

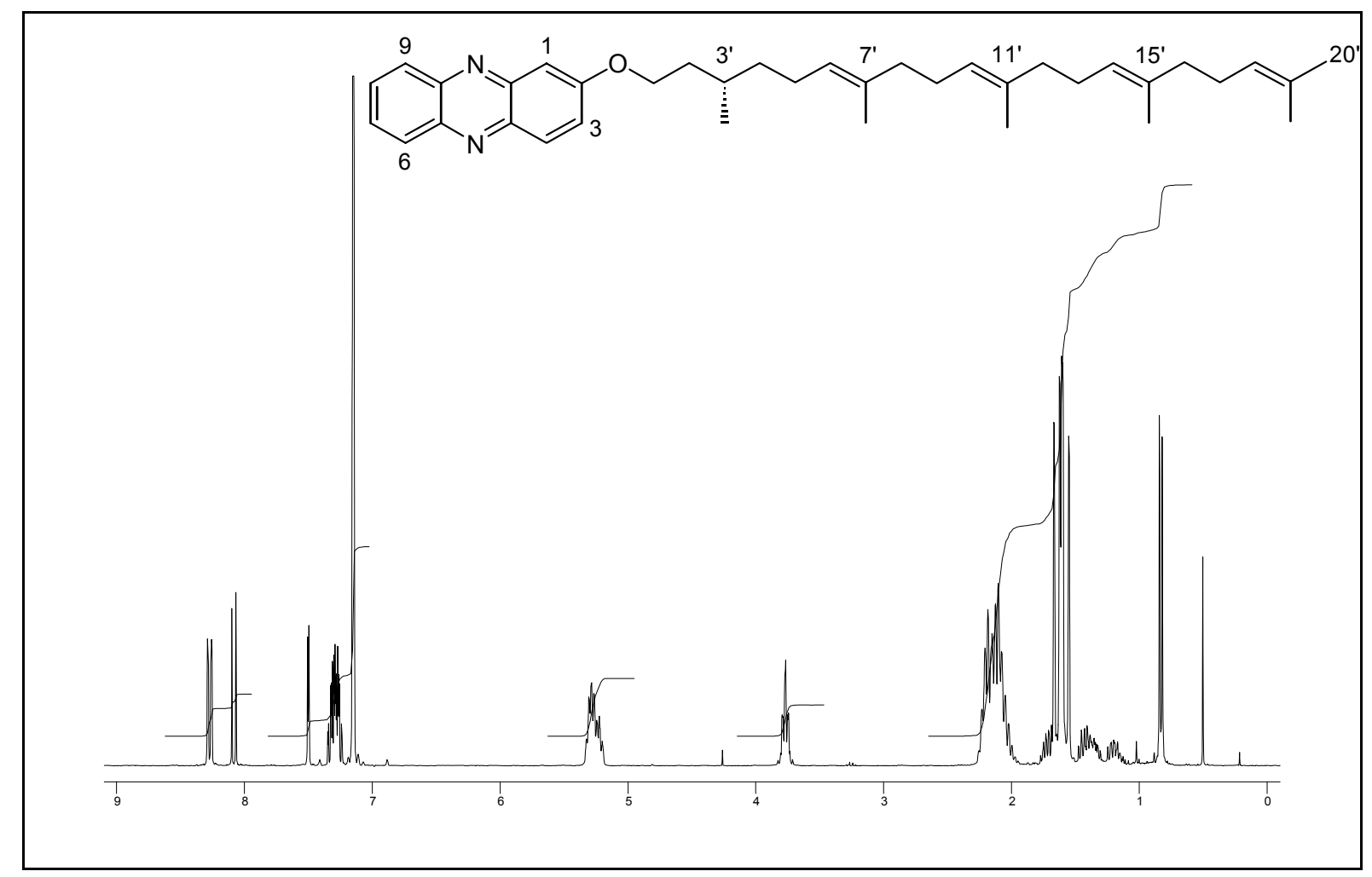

Abb. 57: ${ }^{1} \mathrm{H}$ NMR-Spektrum von synthetischem Methanophenazin $[(S)-1]\left(500 \mathrm{MHz}, \mathrm{C}_{6} \mathrm{D}_{6}\right)$

Die vier olefinischen Protonen 6'-H, 10'-H, 14'-H und 18'-H erscheinen als Multiplett bei $\delta=$ 5.21 bis 5.48 ppm. Die Gegenwart der beiden Stickstoffatome hat den größten Einfluß auf die aromatischen Protonen 4-H, 6-H und 9-H. Ihre Signale sind daher zu tiefem Feld verschoben und finden sich bei $\delta=8.12(4-\mathrm{H})$ und $8.30(6-\mathrm{H}$ und 9-H) ppm. Das Sauerstoffatom hat den stärksten Einfluß auf das 1-H Proton. Es erfährt eine Hochfeldverschiebung und sein Signal tritt bei $\delta=7.53$ ppm, aufgespalten durch die meta-Kopplung mit dem 3-H Proton, als Dublett in Erscheinung. Die Protonen 3-H, 7-H und 8-H zeigen ein Multiplett bei $\delta=7.32$ bis 7.35 ppm. 


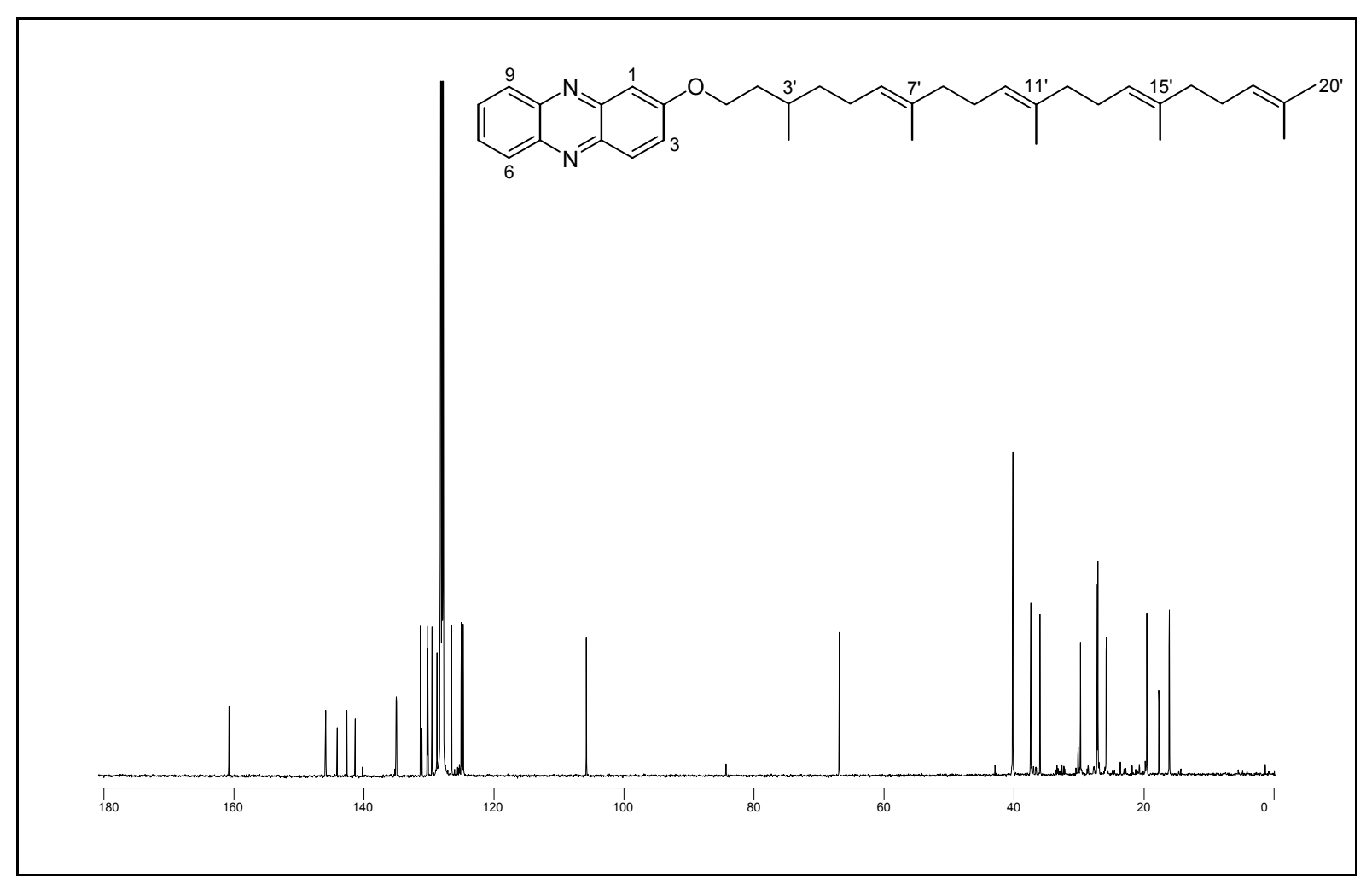

Abb. 58: ${ }^{13} \mathrm{C}$ NMR-Spektrum von natürlichem Methanophenazin (1) (125 MHz, $\mathrm{C}_{6} \mathrm{D}_{6}$ )

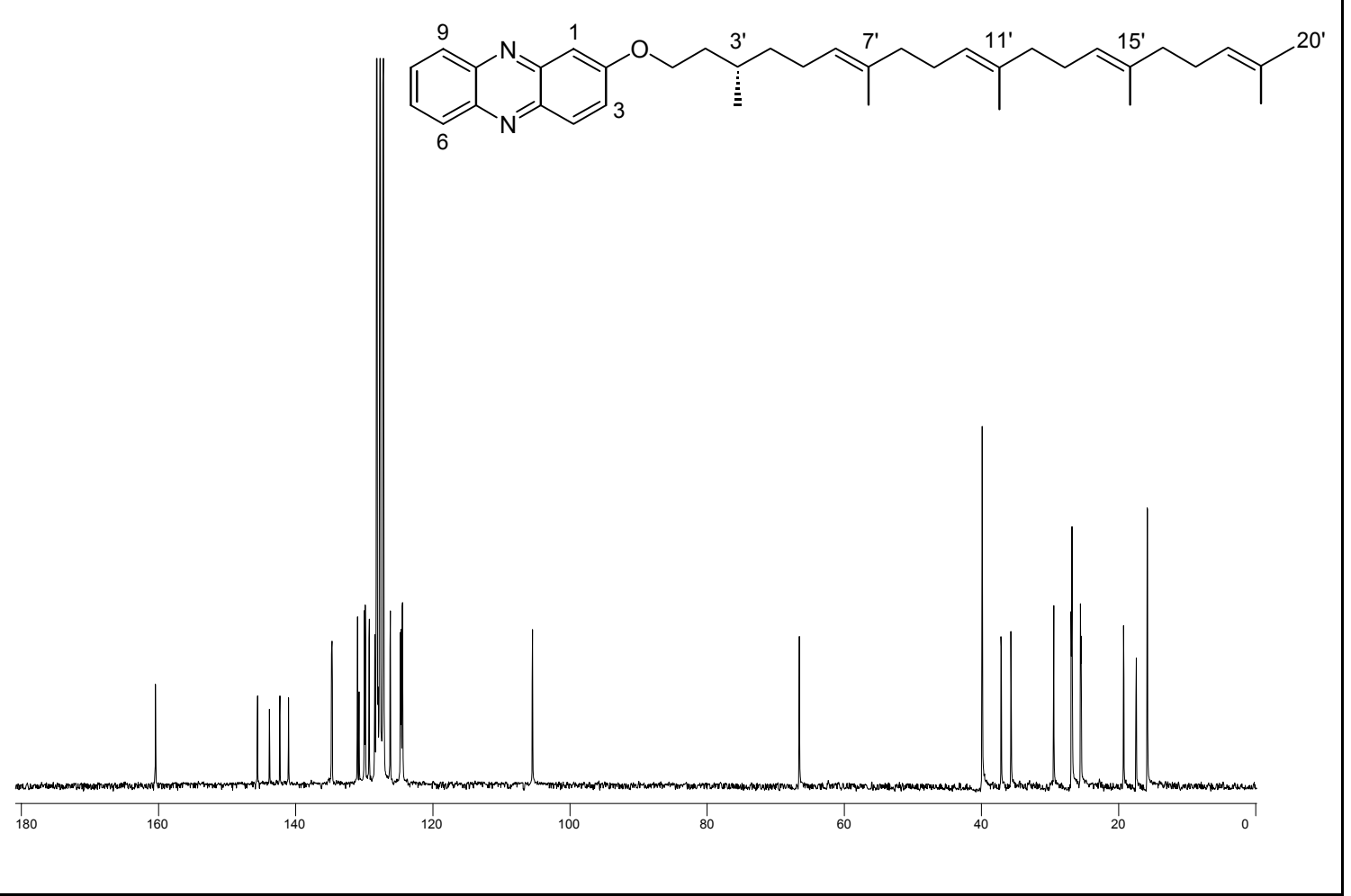

Abb. 59: ${ }^{13} \mathrm{C}$ NMR-Spektrum von synthetischem Methanophenazin $[(S)-1]\left(125 \mathrm{MHz}, \mathrm{C}_{6} \mathrm{D}_{6}\right)$ 
Das ${ }^{13} \mathrm{C}$ NMR-Spektrum von $(S)$-1 präsentiert die Signale der olefinischen Methylgruppen 7'$\mathrm{CH}_{3}, 11^{\prime}-\mathrm{CH}_{3}$, und $15^{\prime}-\mathrm{CH}_{3}$ bei $\delta=16.12,16.13$ und $16.15 \mathrm{ppm}$ (vergleiche Abbildungen 58 und 59). Die Atome 19'- $\mathrm{CH}_{3}$ und C-20' resonieren bei $\delta=17.73$ und $25.78 \mathrm{ppm}$ und markieren zusammen mit der Resonanz des quartären Atoms C-19' bei $\delta=131.07$ das Ende der ungesättigten Seitenkette. Das Signal bei $\delta=19.56 \mathrm{ppm}$ wird der direkt mit dem stereogenen Zentrum verbundenen Methylgruppe 3'- $\mathrm{CH}_{3}$ zugeordnet. Durch die Nähe des Sauerstoffatoms auffallend zu tiefem Feld verschoben, erscheinen die Resonanzsignale der Atome C-1' und C-2 bei $\delta=66.91$ bzw. 160.78 ppm. Ein positiver mesomerer Effekt, hervorgerufen durch das Sauerstoffatom, führt zur Hochfeldverschiebung des Signals von C-1 zu $\delta=105.80 \mathrm{ppm}$. Die quartären aromatischen Atome C-4a, C-5a, C-9a und C-10a stehen in direktem Kontakt mit den beiden elektronegativen Stickstoffatomen und resonieren tieffeldverschoben im Bereich von $\delta=141.36$ bis $145.87 \mathrm{ppm}$. Dadurch lassen sie sich gut von den quartären olefinischen Atomen C-7', C-11' und C-15', deren Signale bei $\delta=134.99$ bis 135.09 ppm erscheinen, unterscheiden.

Im Massenspektrum bildet der Basispeak gleichzeitig das Molekülion und ist bei $\mathrm{m} / \mathrm{z}=538 \mathrm{zu}$ erkennen. Charakteristische Fragmentionen bei $m / z=470,402,334$ und 265 entstehen durch die sukzessive Abspaltung von $\mathrm{C}_{5} \mathrm{H}_{8}$ - bzw. $\mathrm{C}_{5} \mathrm{H}_{9}$-Einheiten durch Allylspaltung aus der Seitenkette.

Insgesamt bleibt festzuhalten, daß die spektroskopischen Daten von synthetischem Methanophenazin rac-1 und (S)-1 mit denen des natürlichen Methanophenazins (1) hervorragend übereinstimmen. Damit ist das Ergebnis der Strukturaufklärung von Methanophenazin (1) durch Totalsynthese bestätigt worden.

\subsection{Zusammenfassung}

Ein Schlüsselschritt zur Synthese von $(S)$-Methanophenazin $[(S)$-1] ist die zum Aufbau der Seitenkette erforderliche Verknüpfung des gesättigten $\mathrm{C}_{6}$-Bausteines $(R)-6$ mit dem ungesättigten $\mathrm{C}_{19}$-Baustein 7. Dies gelingt durch die Überführung des Alkyliodids $(R)-6$ in das entsprechende Zink-Organyl und seine anschließende Palladium-katalysierte Kreuzkupplung mit dem Vinyliodid 7. Desilylieren des Kupplungsprodukts zum Alkohol $(S)$ 78 und Verethern mit 2-Hydroxyphenazin (2) führt zum angestrebten Zielmolekül (S)-1. Unter Verwendung von rac-6 wurde Methanophenazin ( $r a c-1)$ auch in seiner racemischen Form synthetisiert. 


\section{Versuche zur Ermittlung der absoluten Konfiguration von Methanophenazin (1)}

Die einzige ungeklärte Frage im Zusammenhang mit der Strukturaufklärung von natürlichem Methanophenazin (1) ist die nach der absoluten Konfiguration des Naturstoffs. Ihre Klärung wird vor allem durch die geringen zur Verfügung stehenden Mengen des Naturstoffs erschwert.

\subsection{Untersuchungen mit chromatographischen Methoden}

Zur Bestimmung der absoluten Konfiguration eines stereogenen Zentrums bedient man sich häufig der Chromatographie an chiraler Phase. ${ }^{[118]}$ Neben der Verbindung, deren absolute Konfiguration aufgeklärt werden soll, benötigt man dazu am besten das racemische Gemisch und ein Enantiomer mit bekannter absoluter Konfiguration. Dabei etabliert man zunächst mit dem racemischen Gemisch die Bedingungen zur Separation der Enantiomeren. Anschließend vergleicht man die Retentionszeit der Substanz unbekannter Konfiguration mit den Retentionszeiten der Enantiomere bekannter Konfiguration. Die dabei erhaltenen Ergebnisse lassen einen eindeutigen Rückschluß auf die absolute Konfiguration des fraglichen Stereozentrums zu. Durch abschließende Co-Chromatographie der drei Substanzproben überprüft man die erzielten Resultate. Der große Vorteil der chromatographischen Methoden liegt im geringen Substanzbedarf.

Zunächst war geplant, die Konfiguration des stereogenen Zentrums von Methanophenazin (1) durch Gaschromatographie an chiraler Phase zu ermitteln. Bei den Versuchen, die Parameter zur Auftrennung der Enantiomeren von rac-1 zu bestimmen, stellte man schnell die Zersetzung von rac-1 im Injektor des Gaschromatographen fest. Eine Variation der Injektortemperatur im Bereich von 150 bis $250{ }^{\circ} \mathrm{C}$ brachte keinen Fortschritt, da unter keinen Bedingungen aussagekräftige, reproduzierbare Signale detektiert werden konnten.

Hochleistungsflüssigkeitschromatographie war die zweite Methode, die Anwendung fand. ${ }^{[119]}$ An einer speziell für unpolare, chirale Substanzen ausgelegten Säule (Chiralcel OD, Fa. Diacell) wurde versucht, die Trennbedingungen für Methanophenazin ( $r a c-1)$ zu ermitteln. Dabei wurden die folgenden Bedingungen gewählt Flußrate: $0.5 \mathrm{ml} / \mathrm{min}$; Laufmittel: Hexan/Isopropanol mit wechselnden Anteilen von Isopropanol zwischen 1 und 20 \%; UV-Detektion bei $255 \mathrm{~nm}$. Alle Bemühungen zur Auftrennung von rac-1 in die beiden 
Enantiomeren waren ohne Erfolg. Künftige Untersuchungen werden sich jetzt auf den Einsatz weiterer chiraler Phasen konzentrieren.

\subsection{Untersuchung mit chiroptischen Methoden}

Bei den chiroptischen Meßmethoden handelt es sich um eine Form der Absorptionsspektroskopie, die auf der Chiralität der untersuchten Stoffe basiert. Voraussetzung dafür ist das Vorliegen von optischer Aktivität. ${ }^{[108]}$ Ein Stoff ist optisch aktiv, wenn er die Ebene des linear polarisierten Lichtes dreht. Messungen von $(S)$-Methanophenazin $[(S)$-1] im Polarimeter ergaben einen spezifischen Drehwert von $[\alpha]_{546}^{20}=-4.6^{\circ}\left(\mathrm{c}=1, \mathrm{C}_{6} \mathrm{H}_{6}\right)$. Damit erfüllt die Verbindung $(S)$-1 das Kriterium der optischen Aktivität.

Der spezifische Drehwert von natürlichem Methanophenazin (1) wurde nicht bestimmt, weil mit $0.7 \mathrm{mg}$ an 1 zuwenig Substanz vorlag, um eine ausreichend genaue, aussagekräftige Messung durchzuführen. Ein einfacher Vergleich der Drehwerte von natürlichem Methanophenazin (1) mit synthetischem $(S)$-Methanophenazin $[(S)$-1] zur Bestimmung des absoluten Konfiguration war deshalb nicht möglich.

Die Verbindung (S)-1 verfügt über verschiedene Absorptionsmaxima im UV-Spektrum bei $\lambda=256 \mathrm{~nm}, 354$ und $388 \mathrm{~nm}$. Bei geeigneten optisch aktiven Verbindungen werden innerhalb von Absorptionsbanden links und rechts zirkular polarisierte Lichtstrahlen unterschiedlich stark absorbiert. Diese Differenz in der Absorption nennt man Zirkulardichroismus (CD). Er wird mit Hilfe von CD-Spektrometern quantitativ bestimmt. ${ }^{[108]}$ Die zirkulardichroitischen Untersuchungen von (S)-1 an einem Jasco J 715 CD-Spektrometer über einen Wellenlängenbereich von 500 bis $200 \mathrm{~nm}$ wiesen keinen CD-Effekt auf. Die hervorgerufenen Effekte sind offensichtlich zu gering, um einen Unterschied in der Absorption entgegengesetzt zirkular polarisieren Lichtes zu bewirken. Diese Methode eignet sich daher nicht zur Bestimmung der absoluten Konfiguration des Stereozentrums von natürlichem Methanophenazin (1). 


\subsection{Versuche zur Aufklärung der absoluten Konfiguration durch chemischen Abbau}

Ein anderer Weg zur Aufklärung der absoluten Konfiguration von 1 ist der chemische Abbau und die Bestimmung der absoluten Konfiguration des Abbauprodukts. Eine Möglichkeit zur Derivatisierung von $\mathbf{1}$ besteht im ozonolytischen Abbau aller Doppelbindungen der Seitenkette. Durch anschließende reduktive Aufarbeitung der Reaktionsmischung sollte es möglich sein, 1 in den Alkohol 80 überzuführen (Abbildung 60), der dann weiter derivatisiert werden kann.<smiles>CC(C)=CCC/C(C)=C/CC/C(C)=C/CC/C(C)=C/CCC(C)CCOc1ccc2nc3ccccc3nc2c1</smiles>

Abb. 60: geplante Abbaureaktion zur Bestimmung der absoluten Konfiguration von Methanophenazin (1)

Wie in Kapitel 7 dargestellt, wurde der Phenazinether rac-51 durch Veretherung von 2Hydroxyphenazin (2) mit Citronellol (rac-47) synthetisiert. Von M. Dehler ${ }^{[109]}$ durchgeführte Versuche haben gezeigt, daß der Alkohol rac-80 durch ozonolytische Spaltung der terminalen Doppelbindung von $\mathbf{r a c - 5 1}$ und anschließende reduktive Aufarbeitung mit Natriumborhydrid in guten Ausbeuten zugänglich ist (Abbildung 61).<smiles>CC(C)=CCCC(C)CCOc1ccc2nc3ccccc3nc2c1</smiles>

1. $\mathrm{O}_{3}, \mathrm{CH}_{2} \mathrm{Cl}_{2}, \mathrm{MeOH},-78^{\circ} \mathrm{C}$ 2. $\mathrm{NaBH}_{4}, 16 \mathrm{~h}, \mathrm{RT}$

$83 \%$<smiles>CC(CCCO)CCOc1ccc2nc3ccccc3nc2c1</smiles>

Abb. 61: Ozonolyse von rac-51 
Demnach sind auf diesem Weg, ausgehend von 2-Hydroxyphenazin (2) und enantiomerenreinem Citronellol $(R)-47$ bzw. (S)-47, die zur Aufklärung der absoluten Konfiguration von 1 erforderlichen Vergleichssubstanzen $(R)-80$ bzw. (S)-80 leicht zugänglich (Abbildung 62).<smiles>CC(C)=CCC[C@H](C)CCO</smiles>

2
(S)-47
1. $\mathrm{NEt}_{3}, \mathrm{THF}, \mathrm{CH}_{3} \mathrm{SO}_{2} \mathrm{Cl}$

2. $\mathrm{DMF}, \mathrm{K}_{2} \mathrm{CO}_{3}, 100^{\circ} \mathrm{C}$

$80 \%$
1. $\mathrm{O}_{3}, \mathrm{CH}_{2} \mathrm{Cl}_{2}, \mathrm{MeOH},-78^{\circ} \mathrm{C}$

2. $\mathrm{NaBH}_{4}, 16 \mathrm{~h}, \mathrm{RT}$

$76 \%$

(S)-51<smiles>C[C@H](CCCO)CCOc1ccc2nc3ccccc3nc2c1</smiles>

Abb. 62: Synthese und Ozonolyse von (S)-51

Neben den oben genannten Techniken zur Bestimmung der Konfiguration durch chromatographische und chiroptische Methoden bietet die primäre Hydroxylgruppe im Abbauprodukt 80 die Möglichkeit der Derivatisierung mit chiralen Reagenzien und eröffnet somit einen weiteren Weg zur Konfigurationsermittlung durch Kernresonanzspektroskopie.

\subsection{Zusammenfassung}

Versuche zur Bestimmung der absoluten Konfiguration des Naturstoffs Methanophenazin (1) mit chromatographischen und chiroptischen Methoden blieben bislang ohne Erfolg. Eine andere Möglichkeit zur Lösung dieses Problems liegt im chemischen Abbau von 1 durch Ozonolyse der Seitenkette und anschließender Bestimmung der absoluten Konfiguration des Abbauprodukts 80. Die dazu erforderlichen Modellsubstanzen sind aus 2-Hydroxphenazin (2) und enantiomerenreinem Citronellol $(S)-47$ bzw. $(R)-47$ zugänglich. 


\section{Die biologische Funktion von Methanophenazin (1)}

Methanogene Organismen spielen eine zentrale Rolle im Prozeß der Mineralisation organischer Verbindungen (siehe Kapitel 2). Methanosarcina mazei Gö1 ist dabei in der Lage, Abbauprodukte der natürlichen Biopolymere, wie Wasserstoff/Kohlendioxid, Ameisensäure, Methanol und Methylamine in Methan umzuwandeln. ${ }^{[13]}$ Katalysiert wird dieser Vorgang durch die Methyl-Co-M-Reduktase (siehe die Abbildungen 11 und 12 in Kapitel 2.2), wobei Methyl-S-CoM reduktiv zu Methan demethyliert wird. Die dazu erforderlichen Elektronen stammen von HS-CoB. Neben Methan bildet sich dabei das Heterodisulfid CoM-S-S-CoB. Die Reduktion dieses Heterodisulfids ist ein energiekonservierender Schritt im Stoffwechsel methylotropher und hydrogenotropher Methanogener, weil er mit einer Protonentranslokation verbunden ist, die einen transmembranen Protonengradienten aufbaut, der von einer APTase zur ATP-Synthese genutzt wird. ${ }^{[17,18]}$ Verantwortlich für die Regeneration der Coenzyme M (CoM-SH) und B (CoB-SH) in Ms. mazei Gö1 sind zwei membrangebundene Enzymsysteme. Beim Wachstum auf Methanol ist das $\mathrm{F}_{420} \mathrm{H}_{2}:$ Heterodisulfid-Oxidoreduktase-System aktiv. Dabei wird der Elektronentransport von $\mathrm{F}_{420} \mathrm{H}_{2}$ zu CoB-S-S-CoM durch eine $\mathrm{F}_{420} \mathrm{H}_{2}$-Dehydrogenase vermittelt, die die Elektronen mit Hilfe von Elektronenüberträgern auf die HeterodisulfidReduktase überträgt. ${ }^{[26]}$ Nutzt der Organismus Wasserstoff und Kohlendioxid, bedient er sich des $\mathrm{H}_{2}$ :Heterodisulfid-Oxidoreduktase-Systems zum Elektronentransport. Eine membranständige Hydrogenase macht den elementaren Wasserstoff verfügbar und überträgt die zur Heterodisulfid-Reduktion notwendigen Elektronen auf Elektronen-Carrier, die sie zur Heterodisulfid-Reduktase transportieren. ${ }^{[28]}$ Die Frage nach der Natur des Elektronenüberträgers führte zur Extraktion von Cytoplasmamembranen aus Ms. mazei Gö1. Aus dem Rohextrakt wurde Methanophenazin (1) als potentieller Elektronenüberträger isoliert, seine Struktur aufgeklärt und die Verbindung synthetisiert. 


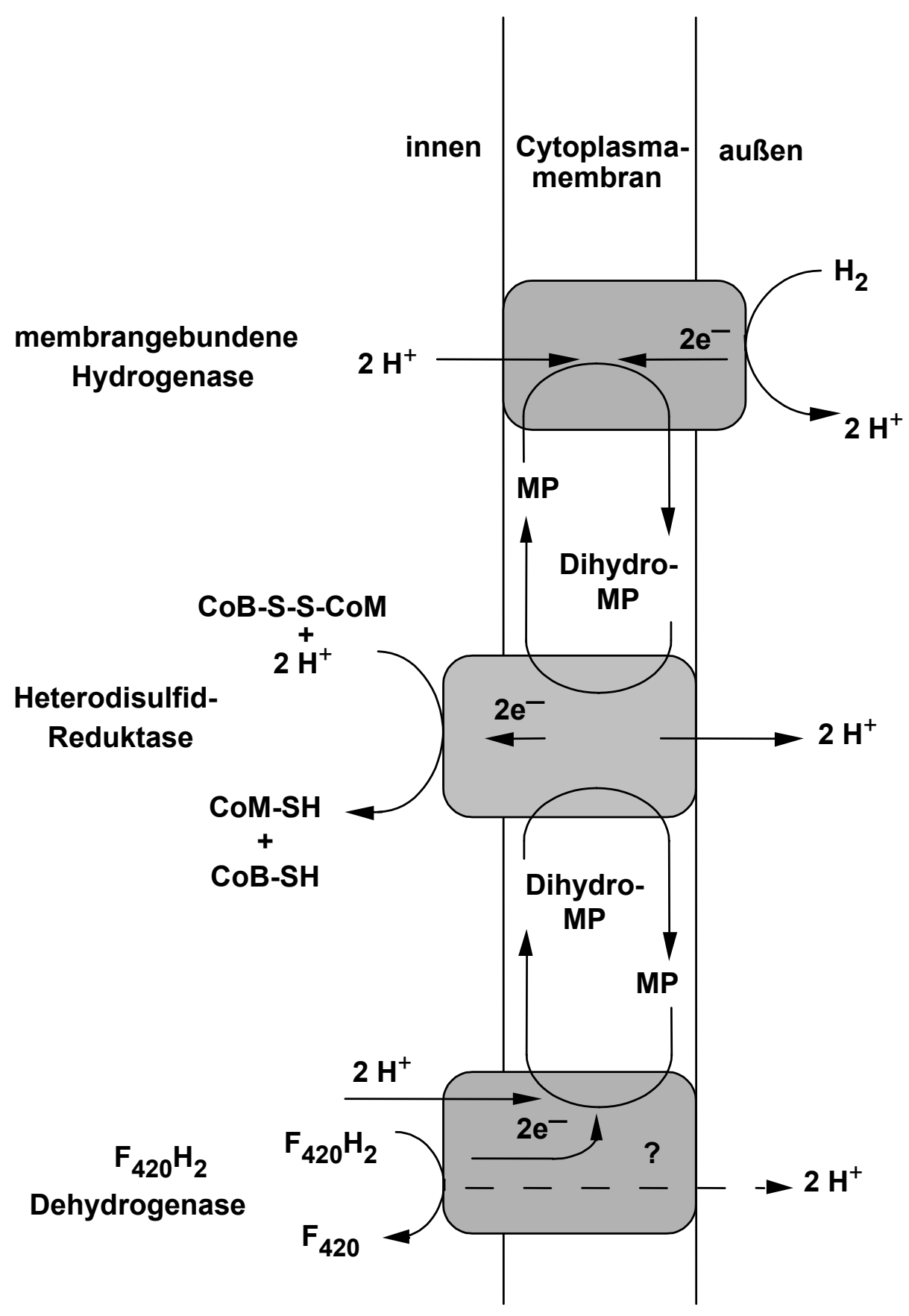

Abb. 63: Modell des membrangebundenen Elektronentransfers von Methanosarcina mazei Gö1 nach Deppenmeier. ${ }^{[29]} \mathrm{CoM}-\mathrm{SH}=$ Coenzym M; CoB-SH = Coenzym B, $\mathrm{F}_{420}=$ Coenzym $\mathrm{F}_{420} ; \mathrm{F}_{420} \mathrm{H}_{2}=$ reduzierte Form des Coenzyms $\mathrm{F}_{420}$; MP = Methanophenazin; Dihydro-MP = reduzierte Form von Methanophenazin ${ }^{[22]}$ 


\subsection{Untersuchungen zum membrangebunden Elektronentransfer in Ms. mazei Gö1}

Die ersten Untersuchungen zur biologischen Funktion wurden von S. Bäumer ${ }^{[28]}$ mit der gut zugänglichen Modellverbindung 2-Hydroxyphenazin (2) durchgeführt. Sie stellt ein um die sesterterpenoide Seitenkette verkürztes Derivat von Methanophenazin (1) dar.

Bei den Versuchen wurden in gepuffertem Medium, in einer Stickstoff-Atmosphäre, $\mathrm{F}_{420} \mathrm{H}_{2}$ und gewaschene Cytoplasmamembranen von Ms. mazei Gö1 vorgelegt. Nach der Zugabe von 2-Hydroxyphenazin (2) wurde die Reaktion spektralphotometrisch verfolgt. Bei einer Wellenlänge von $\lambda=420 \mathrm{~nm}$ beobachtete man eine Zunahme der Absorption, die durch Oxidation von $\mathrm{F}_{420} \mathrm{H}_{2}$ zu $\mathrm{F}_{420}$ hervorgerufen wurde. Gleichzeitig mit der Zunahme der $\mathrm{F}_{420^{-}}$ Absorption beobachtete man eine Abnahme der Phenazin-Absorption bei $\lambda=365 \mathrm{~nm}$, die durch Reduktion von 2-Hydroxyphenazin (2) zu Dehydro-2-hydroxyphenazin (Dehydro-2) hervorgerufen wird. Offensichtlich ist 2 in der Lage, der $\mathrm{F}_{420} \mathrm{H}_{2}$-abhängigen Dehydrogenase als Elektronenakzeptor zu dienen.

Wurden gewaschene Membranen in einer Wasserstoff-Atmosphäre mit 2-Hydroxyphenazin (2) versetzt, so beobachtete man im Photometer eine Abnahme der Absorption als Folge der Reduktion von $2 \mathrm{zu}$ Dehydro-2. Ebenso wie die $\mathrm{F}_{420}$-Dehydrogenase ist es also auch der membranständigen Hydrogenase möglich, Elektronen auf 2 zu übertragen. ${ }^{[28]}$

Zur Überprüfung der Heterodisulfid-Reduktase-Aktivität reduzierte man 2 mit Pt/ $\mathrm{H}_{2}$, filtrierte vom Katalysator ab und mischte das erhaltene Dihydro-2 unter Sauerstoffausschluß mit gewaschen Cytoplasmamembranen. Nach der Zugabe von CoM-S-S-CoB, beobachtete man im Photometer eine Zunahme der Absorption durch die fortlaufende Oxidation von Dihydro-2 zu 2-Hydroxyphenazin (2).

Die in den beiden Elektronentransport-Systemen abgelaufenen Redoxprozesse lassen sich durch die nachfolgend aufgeführten Teilreaktionen beschreiben:

\section{$\mathrm{F}_{420} \mathrm{H}_{2}$ :Heterodisulfid-Oxidoreduktase}
a) $\mathrm{F}_{420} \mathrm{H}_{2}+2$
b) Dihydro-2 + CoB-S-S-CoM
$\mathrm{F}_{420}+$ Dihydro-2
$2+\mathrm{CoB}-\mathrm{SH}+\mathrm{CoM}-\mathrm{SH}$

\section{$\mathrm{H}_{2}$ :Heterodisulfid-Oxidoreduktase}
a) $\mathrm{H}_{2}+2$
b) Dihydro-2 + CoB-S-S-CoM
Dihydro-2
$2+\mathrm{CoB}-\mathrm{SH}+\mathrm{CoM}-\mathrm{SH}$ 
Bei der Wiederholung der genannten Versuche mit gereinigten Enzymen anstelle der gewaschenen Cytoplasmamembranen gelang es, das $\mathrm{F}_{420} \mathrm{H}_{2}$ :Heterodisulfid-OxidoreduktaseSystem funktionell zu rekonstituieren. ${ }^{[110]}$ Das heißt, die beiden Teilreaktionen dieses Elektronentransport-Systems wurden erfolgreich miteinander gekoppelt und sind im selben Reaktionsgefäß im Sinne einer Konsekutivreaktion nacheinander abgelaufen. Der künstliche Elektronenüberträger 2 wird dabei zunächst von der gereinigten $\mathrm{F}_{420} \mathrm{H}_{2}$-abhängigen Dehydrogenase zu Dehydro-2 reduziert und im gleichen Gefäß nach Zugabe von CoB-S-SCoM, unter Katalyse der gereinigten Heteodisulfid-Reduktase, in seine oxidierte Form 2 zurückverwandelt. Versuche zur Rekonstitution des $\mathrm{H}_{2}$ :Heterodisulfid-OxidoreduktaseSystems mit der gereinigten Hydrogenase aus Ms. mazei Gö1 schlugen fehl. Untersuchungen dazu ergaben, daß bei der Aufreinigung dieses Enzyms einige für seine Funktion wichtige Untereinheiten abgetrennt wurden, so daß die Übertragung der Elektronen auf 2 nicht stattfinden konnte.

Nach Abschluß der Totalsynthese von Methanophenazin (rac-1) wurden die mit 2Hydroxyphenazin (2) und gewaschenen Cytoplasmamembranen durchgeführten Versuche von $S$. Bäumer mit rac-1 wiederholt.

Die Ergebnisse der mit $\mathbf{2}$ und rac-1 durchgeführten Versuche sind in Tabelle 6 in übersichtlicher Form zusammengefaßt. Wie daraus $\mathrm{zu}$ ersehen ist, reagieren die Schlüsselenzyme beider Elektronentransportsysteme mit beinahe der gleichen spezifischen Aktivität mit den Elektronenüberträgern 2 und $r a c-1$. Das zeigt an, daß für den Redoxprozeß allein das heteroaromatische Phenazinsystem verantwortlich ist und die terpenoide Seitenkette in rac-1 vermutlich nur zur Verankerung des Elektronenüberträgers in der Membran dient.

Die Ergebnisse zeigen, daß auch Methanophenazin (rac-1) sowohl der $\mathrm{F}_{420}$-abhängigen Dehydrogenase als auch der membrangebunden Hydrogenase als Elektronenakzeptor dient. Außerdem nutzt die Heterodisulfid-Reduktase die reduzierte Form von Methanophenazin (Dihydro-rac-1) als Elektronendonor für die Reduktion von CoB-S-S-CoM (Abbildung 63). 
Tabelle 6: Reaktivität von 2-Hydroxyphenazin (2) und Methanophenazin (rac-1) mit den Komponenten der Elektronentransportsysteme von Methanosarcina mazei Gö1

\begin{tabular}{|c|c|c|c|}
\hline Enzym & Elektronendonor & Elektronenakzeptor & $\begin{array}{c}\text { Spez. Akivität } \\
{\left[U / \text { mg Protein }^{-1}\right]}\end{array}$ \\
\hline $\mathrm{F}_{420} \mathrm{H}_{2^{-}}$ & $\mathrm{F}_{420}$ & 2 & 0.2 \\
\hline \multicolumn{4}{|l|}{ Dehydrogenase } \\
\hline $\mathrm{F}_{420} \mathrm{H}_{2-}$ & $\mathrm{F}_{420}$ & rac-1 & 0.15 \\
\hline \multicolumn{4}{|l|}{ Dehydrogenase } \\
\hline Membrangebundene & $\mathrm{H}_{2}$ & 2 & 3.2 \\
\hline \multicolumn{4}{|l|}{ Hydrogenase } \\
\hline Membrangebundene & $\mathrm{H}_{2}$ & $r a c-1$ & 2.3 \\
\hline \multicolumn{4}{|l|}{ Hydrogenase } \\
\hline $\begin{array}{l}\text { Heterodisulfid- } \\
\text { Reduktase }\end{array}$ & Dihydro-2 & CoB-S-S-CoM & 2.3 \\
\hline $\begin{array}{l}\text { Heterodisulfid- } \\
\text { Reduktase }\end{array}$ & Dihydro-rac-1 & CoB-S-S-CoM & 2.3 \\
\hline
\end{tabular}

Methanophenazin (1) ist also in der Lage, den Elektronentransport zwischen den membrangebundenen Enzymen zu vermitteln. Die mit rac-1 als Elektronenüberträger in den Elektronentransport-Systemen ablaufenden Redoxreaktionen lauten dementsprechend:

\section{$\mathrm{F}_{420} \mathrm{H}_{2}$ :Heterodisulfid-Oxidoreduktase}
a) $\mathrm{F}_{420} \mathrm{H}_{2}+$ rac-1
b) Dihydro-rac-1 + CoB-S-S-CoM
$\mathrm{F}_{420}+$ Dihydro-rac-1
rac-1 + CoB-SH + CoM-SH

\section{$\mathrm{H}_{2}$ :Heterodisulfid-Oxidoreduktase}
a) $\mathrm{H}_{2}+$ rac-1
b) Dihydro-rac-1 + CoB-S-S-CoM

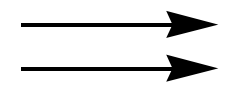
Dihydro-rac-1
rac-1 + CoB-SH + CoM-SH

Damit ist Methanophenazin (1) das erste Phenazin, für das eine Beteiligung am Elektronentransport in biologischen Systemen nachgewiesen wurde. Nach den hier vorgestellten Ergebnissen entspricht die Rolle von Methanophenazin (1) im Energiestoffwechsel methanogener Organismen der des Ubichinons in Mitochondrien und Bakterien. 


\subsection{Zusammenfassung}

Untersuchungen zur biologischen Funktion von 2-Hydroxyphenazin (2) und Methanophenazin ( $r a c-1)$ sowie ihrer reduzierten Formen ergaben, daß diese Verbindungen in der Lage sind, als zentrale redoxaktive Elektronenüberträger in der Cytoplasmamembran von Methanosarcina mazei Gö1 zu fungieren und den Elektronentransport zwischen den Schlüsselenzymen zu vermitteln. Dabei dienen 2 und rac-1 der $\mathrm{F}_{420} \mathrm{H}_{2}$-Dehydrogenase und der membranständigen Hydrogenase als Elektronenakzeptoren. Gegenüber der Heterodisulfid-Reduktase treten die reduzierten Formen Dihydro-2 bzw. Dihydro-rac-1 als Elektronendonoren auf und werden dabei oxidiert. Die Elektronen werden zur Regeneration der Coenzyme $\mathrm{M}$ und $\mathrm{B}$ durch Reduktion des Heterodisulfids CoM-S-S-CoB genutzt. Methanophenazin (1) ist damit das erste Phenazin, für das eine Beteiligung am Elektronentransport in biologischen Systemen nachgewiesen wurde.

Die erzielten Ergebnisse legen nahe, daß die Aufgabe von Methanophenazin (1) im Energiestoffwechsel der Methanogenen der des Ubichinons in Mitochondrien und Bakterien entspricht. 


\section{Zusammenfassung und Ausblick}

Methanogene Organismen gehören zum Reich der Archaea und unterscheiden sich deutlich von den Eukarya und Bakteria. Die ubiquitär verbreiteten methanogenen Archaea sind strikt anaerobe Organismen, die bevorzugt in Sedimenten von Gewässern sowie im Pansen von Wiederkäuern vorkommen. Sie stehen am Ende der anaeroben Nahrungskette und wandeln einfache Substrate wie Wasserstoff/Kohlendioxid, Ameisensäure, Methanol, Methylamine und Essigsäure in Methan um, das anschließend unter aeroben Bedingungen oxidiert werden kann und damit dem Kohlenstoffkreislauf wieder zugeführt wird.

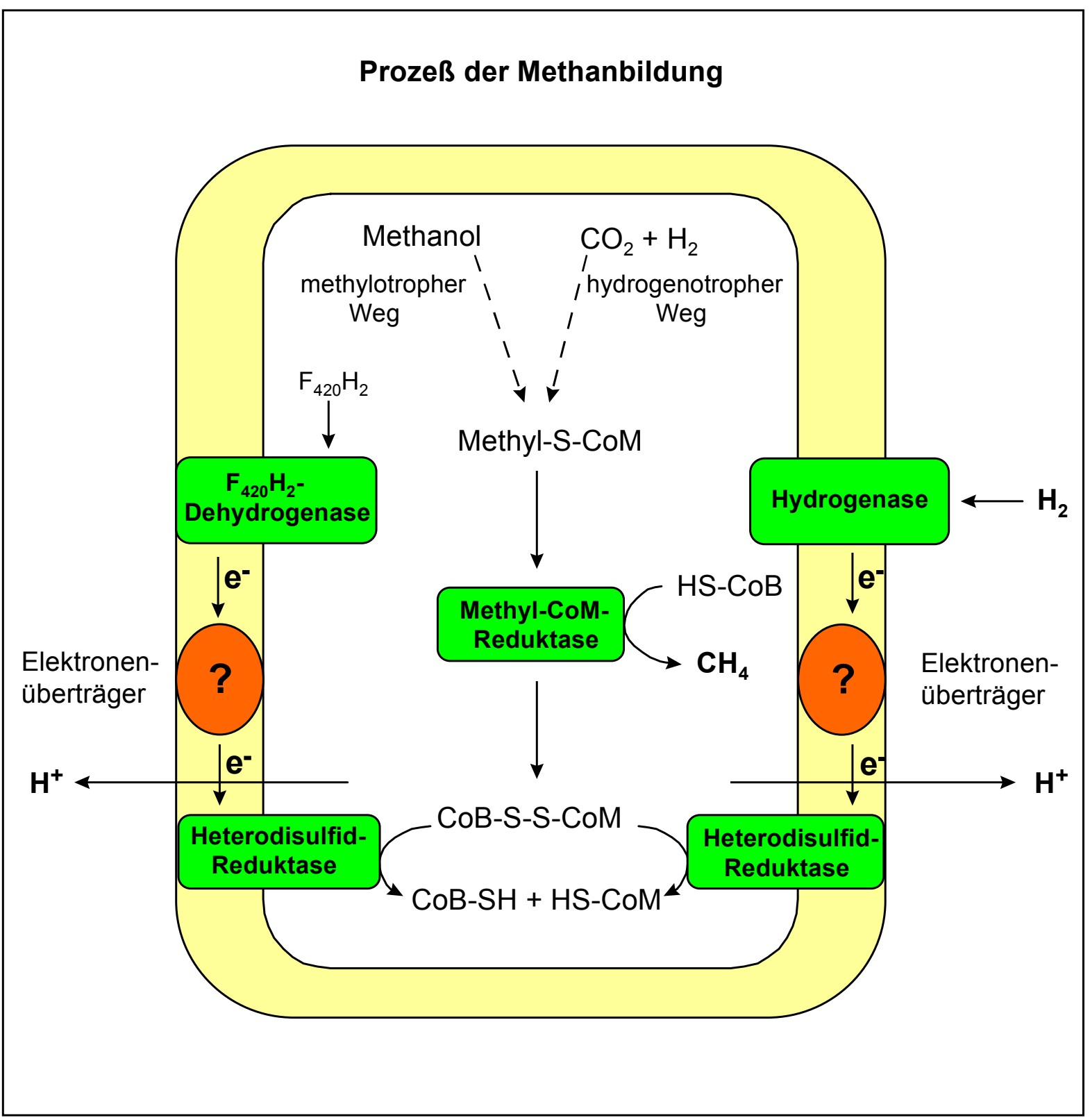

Abb. 64: Vereinfachtes Schema der Methanogenese in Methanosarcina mazei Gö1 
An der Bildung des Methans sind zahlreiche außergewöhnliche Enzyme und ungewöhnliche Cofaktoren beteiligt. Wie in Abbildung 64 dargestellt, ist Methyl-S-CoM die zentrale Zwischenstufe aller methanogenen Stoffwechselwege. Sie wird unter dem katalytischen Einfluß der Methyl-CoM-Reduktase reduktiv zu Methan demethyliert. Die hierfür benötigten Elektronen entstammen CoB-SH und führen zur Bildung eines Heterodisulfids (CoB-S-SCoM) aus CoB-SH und CoM-SH. Ein energiekonservierender Schritt im Stoffwechsel methylotropher und hydrogenotropher Methanogener ist die Reduktion von CoB-S-S-CoM. Am membrangebundenen Elektronentransfer von Methanosarcina mazei Göl sind zwei vor kurzem entdeckte, protonentranslozierende Enzymsysteme beteiligt: die $\mathrm{H}_{2}$ :HeterodisulfidOxidoreduktase und die $\mathrm{F}_{420} \mathrm{H}_{2}$ :Heterodisulfid-Oxidoreduktase. Der Elektronentransport von $\mathrm{F}_{420} \mathrm{H}_{2}$ zu CoB-S-S-CoM wird durch eine $\mathrm{F}_{420} \mathrm{H}_{2}$-Dehydrogenase vermittelt, die die Elektronen mit Hilfe von membrangebundenen Elektronenüberträgern auf die HeterodisulfidReduktase überträgt. Liegt molekularer Wasserstoff vor, fungiert eine membrangebundene Hydrogenase als elektroneneinspeisende Komponente für die Heterodisulfid-Reduktase.

Die Struktur des Elektronenüberträgers war zunächst unbekannt. Auf der Suche danach führte die Extraktion der Cytoplasmamembranen von Methanosarcina mazei Gö1 mit Isooctan und die anschließende Aufreinigung des Rohextrakts mittels HPLC zur Isolierung einer redoxaktiven Verbindung, bei der es sich um einem Phenazinether handelte (Abbildung 65). Die detaillierte NMR-Analyse des nur in geringen Mengen $(1.2 \mathrm{mg}$ aus $5 \times 1001 \mathrm{Kultur})$ erhältlichen, empfindlichen Naturstoffs ergab, daß seine lipophile Seitenkette aus fünf in Kopf-Schwanz-Manier verknüpften isoprenoiden Einheiten besteht. Während die direkt über die Etherbrücke mit dem 2-Phenazinylrest verknüpfte $\mathrm{C}_{5}$-Einheit gesättigt ist, sind die vier weiteren ungesättigt. Drei davon weisen $(E)$-konfigurierte Doppelbindungen auf. Bei dem als Methanophenazin (1) bezeichneten, redoxaktiven Naturstoff handelt es sich um das erste aus Archaea isolierte Phenazin überhaupt.

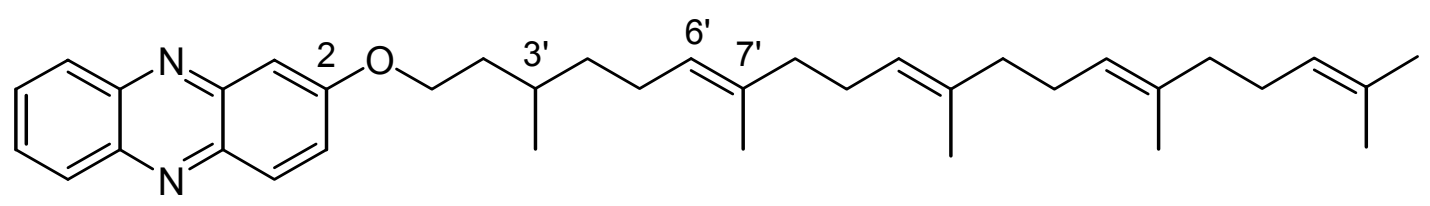

Methanophenazin (1) 
Anzucht von Ms. mazei Gö1 im 100 I Fermenter: 150 mM Methanol, pH 6.9 -7.3, anaerobe

Bedingungen in $\mathrm{N}_{2} / \mathrm{CO}_{2}$-Atmosphäre, 2-3 d, $37^{\circ} \mathrm{C}$

$\Longrightarrow$ Zellmasse: $\approx 100 \mathrm{~g}$<smiles>CCC(C)C</smiles>

Membranpräparation: Abtrennen der Cytoplasmamembranen von Zellwand, DNA und anderen Zellbestandteilen durch Zentrifugation; Isooctanextraktion der Cytoplasmamembranen

$\Rightarrow$ Rohextrakt: $\approx 30 \mathrm{mg}$<smiles>CCC(C)C</smiles>

HPLC-Reinigung des Rohextrakts:

$\mathrm{SiO}_{2}$-Säule; Gradient: Cyclohexan/Essigester, Detektion: $\lambda=260 \mathrm{~nm}$

$\Longrightarrow$ Reinsubstanz: $\approx 0.3 \mathrm{mg}$

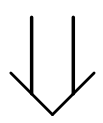

Strukturaufklärung:

Massenspektrometrie, ${ }^{1} \mathrm{H}$ - und ${ }^{13} \mathrm{C}$ NMR-Spektroskopie, zweidimensionale NMR-Korrelationsexperimente<smiles>CCC(C)C</smiles><smiles>CC(C)=CCC/C(C)=C/CC/C(C)=C/CC/C(C)=C/CCC(C)CCOc1ccc2nc3ccccc3nc2c1</smiles>

\section{Methanophenazin (1)}

das erste Phenazinderivat aus Archaea

Abb. 65: Schema zur Isolierung, Reinigung und Strukturaufklärung von Methanophenazin (1) aus Cytoplasmamembranen von Methanosarcina mazei Gö1 
Da Methanogene keine herkömmlichen Chinone besitzen, wurde vermutet, daß $\mathbf{1}$ als Elektronenüberträger in der Cytoplasmamembran fungiert und eine Rolle im energiekonservierenden Elektronentransport übernimmt.

Zum Zwecke einer Prüfung der biologischen Funktion von 1 und dem genauen Studium seiner Rolle im membrangebundenen Elektronentransport von Ms. mazei sowie zur Aufklärung der absoluten Konfiguration an C-3' waren größere Mengen des Naturstoffs erforderlich. Die durch Extraktion aus Cytoplasmamembranen verfügbaren Mengen an Methanophenazin (1) sind zu gering und der Aufwand ihrer Isolierung ist zu groß, als daß sich der Bedarf an 1 aus dieser natürlichen Quelle decken ließe. Eine Synthese des Naturstoffs war also unumgänglich.<smiles>CC(C)=CCC/C(C)=C/CC/C(C)=C/CC/C(C)=C/CC[C@@H](C)CCOc1ccc2nc3ccccc3nc2c1</smiles>
rac-1<smiles>Oc1ccc2nc3ccccc3nc2c1</smiles>

2<smiles>CC(C)C(C)C</smiles>

5<smiles>CCC(C)C</smiles><smiles>[3H-]</smiles><smiles>COCCC(C)CCC=C(C)CCC=C(C)CCC=C(C)CCC=C(C)C</smiles>

rac-3<smiles>CCC(C)C</smiles><smiles>CC=C(C)CCC=C(C)C</smiles><smiles>CCCC(C)=CCCC(C)=CI</smiles>

rac-6

7<smiles>CC(C)=CCCC(C)=CCCC(C)=CCCC(C)C</smiles>

10

Abb. 66: Zur Retrosynthese von Methanophenazin (rac-1) 
Wie in Abbildung 66 dargestellt, führt die retrosynthetische Analyse des racemischen Methanophenazins ( $r a c-1)$ zu den drei Bausteinen 2, rac-6 und 7. Das 2-Hydroxyphenazin (2) soll durch Umsetzung von 4 mit 5 hergestellt werden. Der ungesättigte Teil der Seitenkette von 1 soll durch das Vinyliodid (7) eingeführt werden, das sich auf das bekannte $(E, E)$ Farnesylaceton (10) zurückführen läßt. Das mittlere Fragment rac-6 kann aus 3-Methylpentan-1,5-diol (8) durch selektive Funktionalisierung der beiden Hydroxylgruppen zugänglich gemacht werden. Der Vorteil eines solchen symmetrischen $\mathrm{C}_{6}$-Bausteins besteht in der Möglichkeit, durch Differenzierung seiner enantiotopen Liganden beide Enantiomeren verfügbar zu machen. So ist der kommerziell in hoher optischer Reinheit (ee > 95\%) erhältliche $(R)$-3-Methylglutarsäureester [(R)-11] ein geeigneter Vorläufer zum Aufbau der chiralen Lactone $(R)-\mathbf{1 2}$ und $(S)$-12. Aus ihnen sollten die beiden Bausteine $(R)-\mathbf{6}$ und $(S)-6$ zugänglich sein, die die Grundlage für den Aufbau der beiden Antipoden $(S)$-1 und $(R)-\mathbf{1}$ bilden (Abbildung 67).<smiles>CC(CCO)CCI</smiles>

(R)-6<smiles>CC(C)C(C)C</smiles><smiles>CC1CCOC(=O)C1</smiles>

$(R)-12$

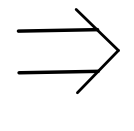

$\mathrm{HO}$<smiles>CCOC(=O)CC(C)CC(=O)O</smiles>

$(R)-11$<smiles>CC(CCO)CCI</smiles><smiles>[AsH3]</smiles><smiles>CCCCC(C)C</smiles>

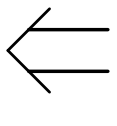<smiles>CC1CCOC(=O)C1</smiles>

(S)-12

Abb. 67: Zur Retrosynthese der enantiomeren lodalkohole (R)-6 und (S)-6 aus (R)-11

Als Schlüsselschritte der Synthese racemischen Methanophenazins (rac-1) gelten der diastereoselektive Aufbau des isoprenoiden Vinyliodids (7) und dessen Verknüpfung mit rac6 zur vollständigen Seitenkette rac-3. Im letzten Schritt sollen rac-3 und 2-Hydroxyphenazin (2) durch Veretherung ins Zielmolekül rac-1 übergeführt werden. 


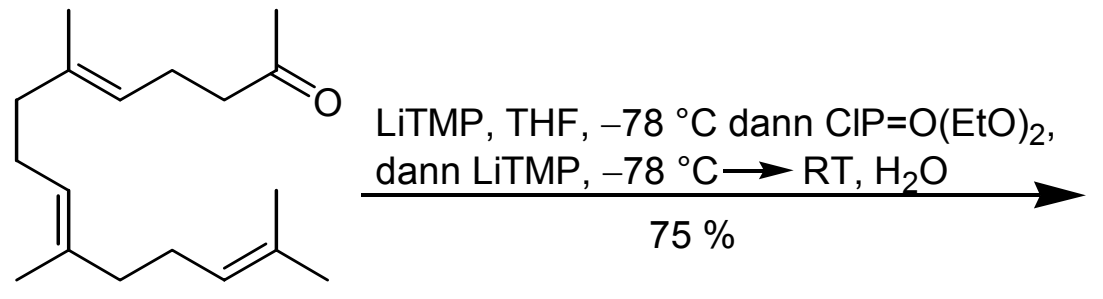

10<smiles>C#CCC/C=C(\C)CC/C=C(/C)CCC=C(C)C</smiles>

9

1. $\mathrm{Cp}_{2} \mathrm{ZrCl}_{2}, \mathrm{Me}_{3} \mathrm{Al}, \mathrm{CH}_{2} \mathrm{ClCH}_{2} \mathrm{Cl}$

2. $\mathrm{I}_{2}, \mathrm{THF},-40{ }^{\circ} \mathrm{C} \rightarrow 0^{\circ} \mathrm{C}$ $74 \%$<smiles>CC(C)=CCC/C(C)=C/CC/C(C)=C/CC/C(C)=C/I</smiles>

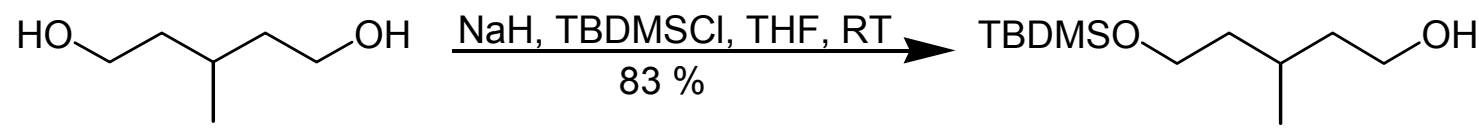

8

rac-52

1. $\mathrm{CH}_{2} \mathrm{Cl}_{2}, \mathrm{NEt}_{3}, \mathrm{CH}_{3} \mathrm{SO}_{2} \mathrm{Cl}, 0{ }^{\circ} \mathrm{C}$

2. Nal, Aceton, $55^{\circ} \mathrm{C}$<smiles>COS(=O)(=O)OCCC(C)CCI</smiles>

rac-6

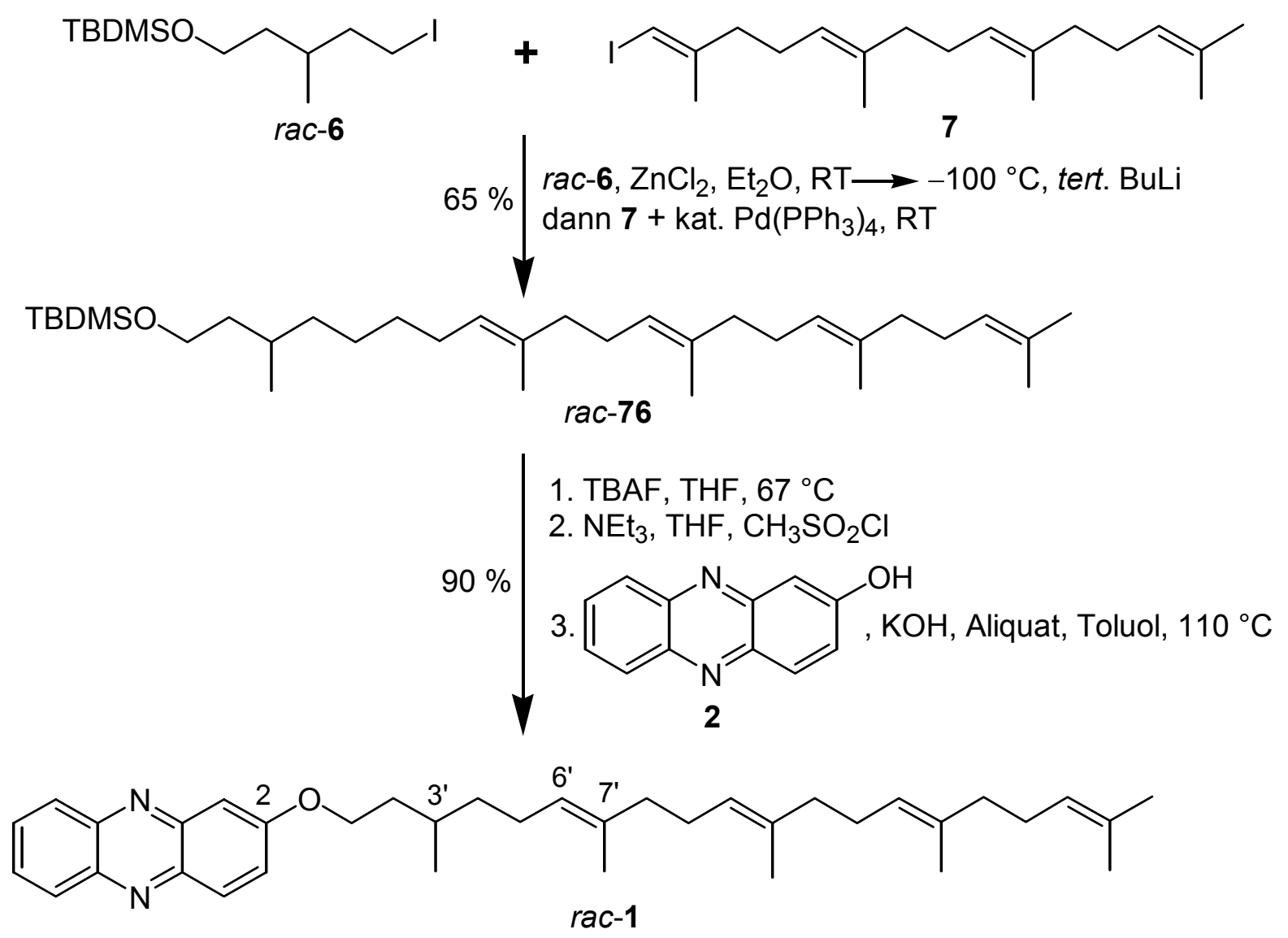

Abb. 68: Zur Synthese von racemischen Methanophenazin (rac-1) 
2-Hydroxyphenazin (2) wurde nach einer Vorschrift von Kehrmann und Cherpillod durch die Kondensation von 1,2,4-Trihydroxybenzol (45) mit $o$-Phenylendiamin (5) synthetisiert.<smiles>Oc1ccc(O)c(O)c1</smiles>

45
1. $\mathrm{Ag}_{2} \mathrm{O}, \mathrm{Et}_{2} \mathrm{O}, \mathrm{RT}$

2. o-Phenylendiamin (5), AcOH, RT $18 \%$<smiles>Oc1ccc2nc3ccccc3nc2c1</smiles>

2

Zur Herstellung von 7 wurde $(E, E)$-Farnesylaceton (10) zunächst in das terminale Alkin 9 transformiert und dann durch eine Zirkonium-katalysierte Carboaluminierung mit Trimethylaluminium und Abfangen mit Iod in das diastereomerenreine (E)-Vinyliodid (7) mit $74 \%$ Ausbeute übergeführt (Abbildung 68).

Das zur Verknüpfung mit 7 erforderliche Alkyliodid rac-6 konnte durch selektive Monofunktionalisierung von 3-Methyl-pentan-1,5-diol (8) in wenigen Schritten zugänglich gemacht werden. Die Umwandlung von rac-6 in die entsprechende Organozinkverbindung durch Behandeln mit trockenem Zinkchlorid und tert.-Butyllithium ermöglichte die Palladium-katalysierte $\mathrm{sp}^{2}$-sp $\mathrm{sp}^{3}$-Kreuzkupplung mit dem Vinyliodid $7 \mathrm{zu}$ rac-76. Diese C-CKupplung verlief unter Retention der Konfiguration am $\mathrm{sp}^{2}$-Zentrum und gestattet den $(E)$ selektiven Aufbau der C-6', C7'-Doppelbindung der Seitenkette rac-76 mit 65 \% Ausbeute. Fluoridinduzierte Silyletherspaltung und die Aktivierung der so gebildeten Hydroxylgruppe durch Methansulfonsäurechlorid ergab das Mesylat, das mit 2-Hydroxyphenazin (2) zu rac-1 verethert wurde.

Durch die konvergente Synthesestrategie gelang es, Methanophenazin (rac-1) in racemischer Form ausgehend von $(E, E)$-Farnesylaceton (10) in 6 Schritten mit einer Gesamtausbeute von $32 \%$ zu synthetisieren.

Für die Synthese von $(S)$ - bzw. $(R)$-Methanophenazin $[(R)$ - bzw. $(S)-1]$ wurde eine stereodivergente Synthesestrategie, die auf der bekannten Umwandlung von $(R)-\mathbf{1 1}$ in die Lactone $(R)$-12 und $(S)$-12 beruhte, ausgearbeitet.

Wie in Abbildung 69 dargestellt, ließen sich ausgehend von $(R)-3$ Methylglutarsäureethylester $[(R)-11]$ (ee > 95\%) durch chemoselektive Reduktion der Carbonsäure- bzw. der Ester-funktionalität die beiden enantiomeren Lactone $(S)$-12 und $(R)$ 12 synthetisieren. Letztere Verbindung erhielt man dabei mit einer Ausbeute von $92 \%$ und 
einem Enantiomeren-überschuß von 98 \%. Die Öffnung des Lactons $(R)-\mathbf{1 2}$ mit Piperidin zum entsprechenden Amidalkohol und dessen Silylierung ergaben nahezu quantitativ das Amid $(R)-57$. Die einstufige Reduktion des tertiären Amids $(R)$-57 zum primären Alkohol $(S)-52$ gelang mit Lithiumtriethylborhydrid in einer Ausbeute von $92 \%$.

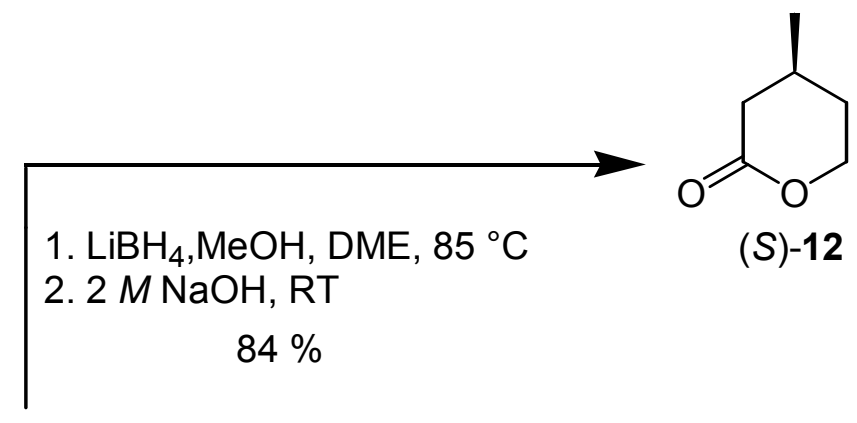<smiles>CCOC(=O)CC(C)CC(=O)O</smiles>

$(R)-11$

1. $\mathrm{BH}_{3} \bullet \mathrm{SMe}_{2}, \mathrm{THF}, 0^{\circ} \mathrm{C} \rightarrow \mathrm{RT}$,

2. $2 \mathrm{M} \mathrm{KOH}, \mathrm{H}_{2} \mathrm{O} / \mathrm{MeOH}, \mathrm{RT}$

$92 \%$<smiles>C[C@H]1CCOC(=O)C1</smiles>

$(R)-12$
1. Piperidin, $12 \mathrm{~h}, 70{ }^{\circ} \mathrm{C}$

2. TBDMSCl, $\mathrm{NEt}_{3}$, $\mathrm{CH}_{2} \mathrm{Cl}_{2}, \mathrm{RT}$ $96 \%$

TBDMSO
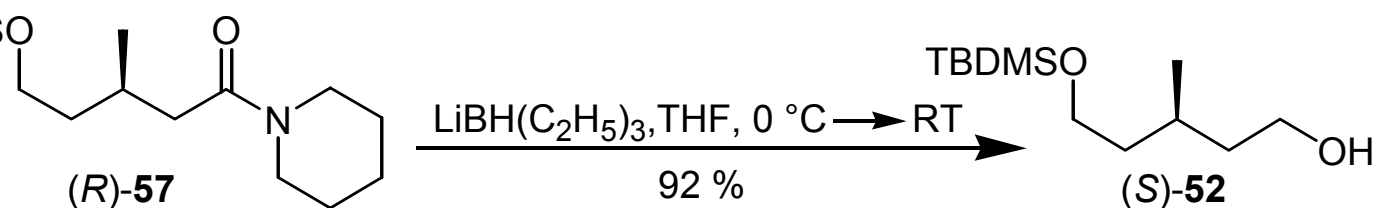

1. $\mathrm{THF}, \mathrm{NEt}_{3}, \mathrm{CH}_{3} \mathrm{SO}_{2} \mathrm{Cl}, 0^{\circ} \mathrm{C}$ 2. THF, Lil, $67^{\circ} \mathrm{C}$ $93 \%$<smiles>CCCCOCCC(C)CCI</smiles>

$(R)-6$

Abb. 69: Zur Synthese von (R)-6 und (S)-12

Aktivieren der Hydroxylfunktion mit Methansulfonsäurechlorid und Reaktion mit Lithiumiodid lieferte den $\mathrm{C}_{6}$-Baustein $(R)$-6. Im Ergebnis gelang die Synthese des Alkyliodids $(R)-6$ aus $(R)-11$ in fünf Schritten mit einer Gesamtausbeute von $76 \%$. Die Integrität des stereogenen Zentrums bleibt dabei unangetastet. 
In Analogie zur Synthese von racemischem Methanophenazin (rac-1) wurde durch eine Palladium-katalysierte C-C-Kreuzkupplung des enantiomerenreinen Bausteins $(R)-6$ mit $(E)$ Vinyliodid 7 und anschließender Desilylierung des Kupplungsproduktes der Alkohol $(S)$-78 hergestellt (Abbildung 70). Die abschließende Veretherung mit 2-Hydroxyphenazin (2) ergab $(S)$-Methanophenazin $[(S)-1]$ mit $90 \%$ Ausbeute.

Da sich $(S)$-12 leicht durch chemoselektive Reduktion der Esterfunktionalität von $(R)-\mathbf{1 1}$ und anschließende Cyclisierung darstellen läßt, ist mit der hier vorgestellten Strategie auch die Grundlage für die Synthese von $(R)$-Methanophenazin $[(R)-1]$ gelegt worden.

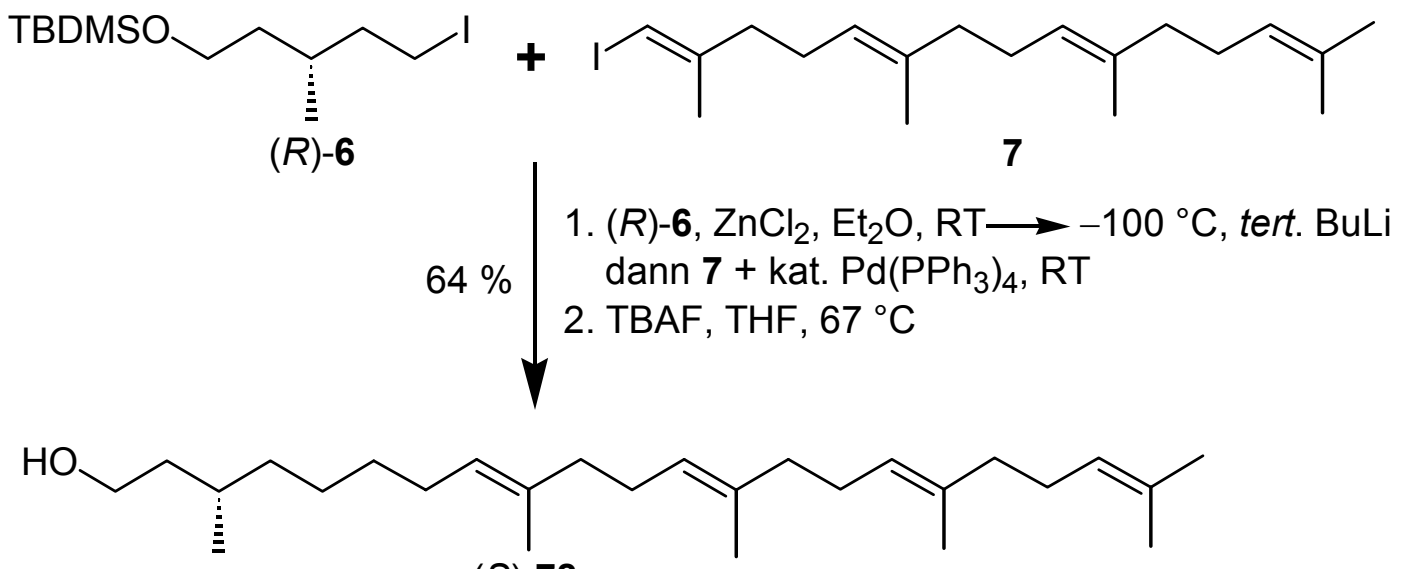

$(S)-78$

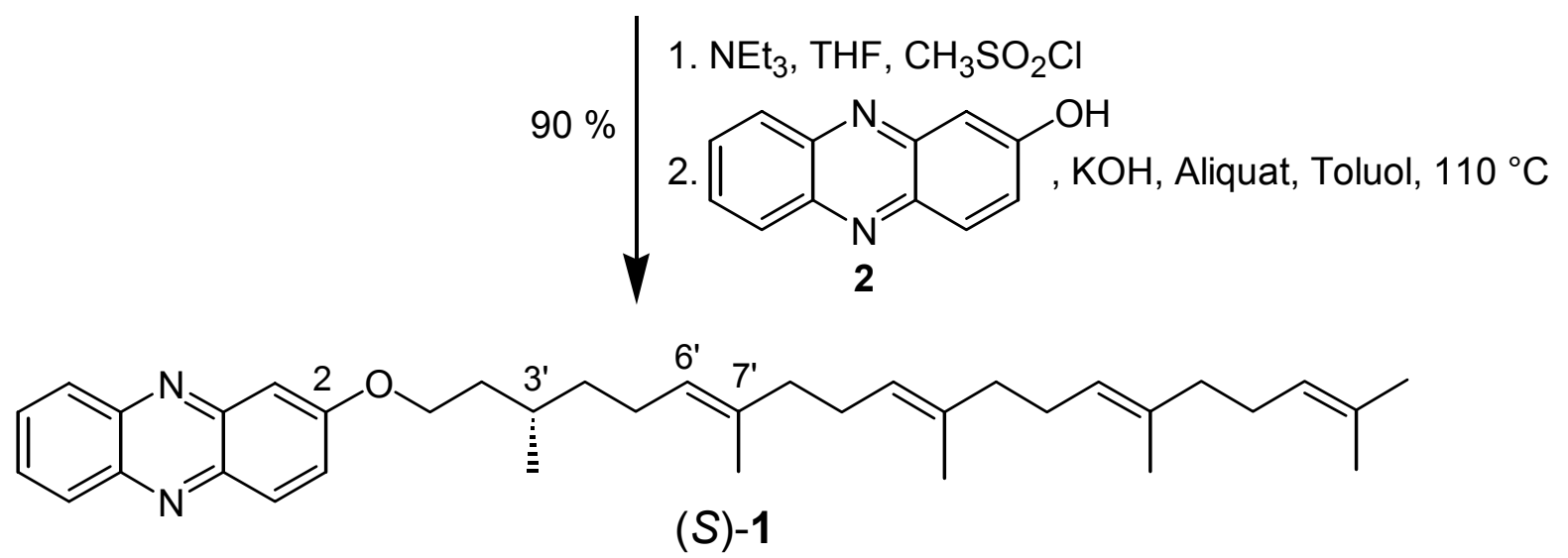

Abb. 70: Zur Synthese von (S)-Methanophenazin [(S)-1]

Ausgehend von den leicht zugänglichen Substraten 2-Hydroxyphenazin (2), 3-Methyl-1,5pentandiol (8) und (E,E)-Farnesylaceton (7), stellt die vorliegende Synthese einen effizienten und konvergenten Zugang zu Methanophenazin (1) dar. Die Möglichkeit der gezielten Manipulation der drei Bausteine, eröffnet auch einen einfachen Weg zur Darstellung von Derivaten von 1. Unter Verwendung des enantiomerenreinen Alkyliodids $(R)$-6, das sich aus 
$(R)$-3-Methylglutarsäureethylester $[(R)$-11] (ee > 95\%) aufbauen läßt, wurde die Synthese von $(S)$-Methanophenazin $[(S)$-1] verwirklicht. Der Antipode $(R)$-Methanophenazin $[(R)-1]$ wird sich nach derselben Strategie synthetisieren lassen, da sich $(R)-\mathbf{1 1}$ auch in das Lacton $(S)-\mathbf{1 2}$ umwandeln läßt, das eine Vorstufe für das Iodid $(S)-6$ darstellt.

Zukünftige Untersuchungen werden sich auf die Bestimmung der absoluten Konfiguration des natürlichen Methanophenazins (1) konzentrieren. Nach chiroptischen und chromatographischen Verfahren werden dabei chemische Abbaureaktionen im Mittelpunkt stehen, die bereits an Modellverbindungen erfolgreich durchgeführt worden sind.

Die ersten Experimente zur biologischen Funktion von Phenazinen bei der Methanogenese von Archaea wurden mit 2-Hydroxyphenazin (2) und seiner reduzierten Form (Dihydro-2) durchgeführt (AK U. Deppenmeier). Sie zeigten, daß die in den Elektronentransport involvierten Enzyme des $\mathrm{F}_{420} \mathrm{H}_{2}$ :Heterodisulfid-Oxidoreduktase-Systems und des $\mathrm{H}_{2}$ :Heterodisulfid-Oxidoreduktase-Systems mit dem künstlichen Elektronenüberträger reagieren. Entsprechende Untersuchungen mit synthetischem Methanophenazin (rac-1) wurden an gewaschenen Cytoplasmamembranen von Methanosarcina mazei Gö1 durchgeführt. Die photometrische Bestimmung der Enzymaktivitäten ergab, daß die membrangebundene Hydrogenase in Gegenwart von Wasserstoff, bzw. die $\mathrm{F}_{420^{-}}$ Dehydrogenase in Gegenwart von $\mathrm{F}_{420} \mathrm{H}_{2}$ in der Lage ist, Elektronen auf rac-1 zu übertragen. Im weiteren Verlauf fungiert die reduzierte Form von rac-1 (Dihydro-rac-1) als Elektronendonor für die Heterodisulfid-Reduktase und ermöglicht die HeterodisulfidReduktion. Demnach ist rac-1 in der Lage, den Elektronentransport zwischen den membrangebunden Enzymen zu vermitteln. Die dabei ablaufenden Redoxprozesse lassen sich in zwei Teilreaktionen beschreiben.

\section{$\mathrm{F}_{420} \mathrm{H}_{2}$ :Heterodisulfid-Oxidoreduktase}
a) $\mathrm{F}_{420} \mathrm{H}_{2}+$ rac-1
b) Dihydro-rac-1 + CoB-S-S-CoM

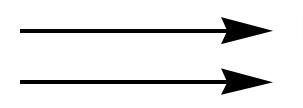
$\mathrm{F}_{420}+$ Dihydro-rac-1
rac-1 + CoB-SH + CoM-SH

\section{$\mathrm{H}_{2}$ :Heterodisulfid-Oxidoreduktase}
a) $\mathrm{H}_{2}+r a c-1$
b) Dihydro-rac-1 + CoB-S-S-CoM
Dihydro-rac-1
rac-1 + CoB-SH + CoM-SH 
Methanophenazin (1), über dessen Isolierung, Strukturaufklärung und Totalsynthese in dieser Arbeit berichtet wird, ist das erste Phenazin aus methanogenen Archaea. Es stellt gleichzeitig das erste am Elektronentransport in biologischen Systemen beteiligte Phenazin-Derivat dar. Die bisherigen Ergebnisse zeigen, daß die Funktion von 1 im Energiestoffwechsel der Methanogenen mit der des Ubichinons in Mitochondrien und Bakterien vergleichbar ist.

Weitere Arbeiten werden sich mit dem Mechanismus der Elektronentransferreaktion von Phenazinen in natürlichen Systemen, aber auch in Modellen beschäftigen. 


\section{Experimenteller Teil}




\section{$1 \quad$ Allgemeine Verfahren}

\subsection{Allgemeine Arbeitstechniken}

Alle sauerstoff- und feuchtigkeitsempfindlichen Reaktionen wurden unter einem leichten Überdruck von Argon in ausgeheizten, trockenen Glasapparaturen unter Anwendung von Spritzen- und Septentechniken durchgeführt. Die hierbei verwendeten Lösungsmittel wurden zuvor destilliert und entsprechend den üblichen Laboratoriumsmethoden absolutiert. ${ }^{[111]}$ Käufliche Substanzen wurden ohne weitere Reinigung eingesetzt.

\subsection{Verwendete Geräte}

Infrarotspektren: Verwendet wurden ein FT-IR Spektrometer IFS 25 der Firma Bruker. Kristalline Substanzen wurden als KBr-Preßlinge, flüssige Proben als Film zwischen NaClPlatten gemessen. Zur Eichung diente eine Polystyrolbande bei $1601 \mathrm{~cm}^{-1}$.

UV/VIS-Spektren: Verwendet wurden die Spektrometer Cary 219 der Firma Varian und die Modelle Lambda 2 bzw. Lambda 9 der Firma Perkin-Elmer.

${ }^{1}$ H NMR-Spektren: Die ${ }^{1}$ H NMR-Spektren wurden an den Modellen XL-200 (200 MHz), VXR (200 MHz) und VXR-500 S (500 MHz) der Firma Varian sowie dem Modell AMX-300 (300 MHz) der Firma Bruker aufgenommen. Die chemischen Verschiebungen sind in Einheiten der $\delta$-Skala angegeben. Tetramethylsilan $\left(\delta_{\mathrm{TMS}}=0.00 \mathrm{ppm}\right)$, Chloroform $\left(\delta_{\mathrm{CHCl}_{3}}=\right.$ $7.24 \mathrm{ppm})$ und Benzol $\left(\delta_{\mathrm{C}_{6} \mathrm{H}_{6}}=7.26 \mathrm{ppm}\right)$ dienten als interne Standards. Zur Kennzeichnung der Multiplizität der Signale werden folgende Abkürzungen verwendet: $s=$ Singulett, $d=$ Dublett, $\mathrm{t}=$ Triplett, $\mathrm{q}=$ Quartett, quint $=$ Quintett, sept $=$ Septett, $\mathrm{dd}=$ Dublett vom Dublett, $\mathrm{dt}=$ Dublett vom Triplett, $\mathrm{m}=$ Multiplett, weiterhin $\mathrm{m}_{\mathrm{C}}$ für das Zentrum eines symmetrischen Multipletts und br für breite Signale. Die Spektren wurden den Regeln erster Ordnung entsprechend interpretiert. Die Kopplungskonstanten ${ }^{n} J_{\text {X-H, Y-H }}$ sind in Hertz (Hz) angegeben. Der Index $n$ gibt die Art der Kopplung an: ${ }^{2} J$ entspricht einer geminalen Kopplung, ${ }^{3} J$ einer vicinalen Kopplung. Bei $\mathrm{n}>3$ handelt es sich um eine Fernkopplung. $\mathrm{X}$ und $\mathrm{Y}$ geben die $\mathrm{C}$ Atome an, deren Protonen miteinander koppeln. 
${ }^{13}$ C NMR-Spektren: Verwendet wurden die Modelle XL-200 (50.3 MHz), VXR-200 (50.3 MHz) und VXL-500 S (125.7 MHz) der Firma Varian sowie das Modell AMX-300 (75.4 $\mathrm{MHz})$ der Firma Bruker. Tetramethylsilan $\left(\delta_{\mathrm{TMS}}=0.00 \mathrm{ppm}\right)$, Chloroform $\left(\delta_{\mathrm{CHCl}_{3}}=7.24\right.$ ppm) und Benzol $\left(\delta_{\mathrm{C}_{6} \mathrm{H}_{6}}=7.26 \mathrm{ppm}\right)$ dienten als interne Standards. Die chemischen Verschiebungen sind ${ }^{1} \mathrm{H}$-breitband-entkoppelten Spektren entnommen. Die '+' $/$ '- Zeichen vor den Werten der chemischen Verschiebung geben die Orientierung der Signale in den ${ }^{13} \mathrm{C}$ NMR APT-Spektren an. Ein '+' Zeichen repräsentiert dabei das Signal einer $\mathrm{CH}$ - oder $\mathrm{CH}_{3}$ Resonanz, ein `-' Zeichen steht für das Signal einer $\mathrm{CH}_{2}$ - oder $\mathrm{C}_{\text {quart- }}$ Resonanz.

Massenspektren: Es wurden die Modelle MAT 311A (niederaufgelöste Spektren) und MAT 731 (hochaufgelöste Spektren) der Firma Varian eingesetzt. Die in Klammern gesetzten Prozentzahlen geben die Intensitäten der Peaks bezogen auf den Basispeak $(I=100 \%)$ an.

Die zur GC-MS Kopplung notwendigen gaschromatographischen Trennungen wurden an einer DB-5 Kapillarsäule (20 m, 0.25 mm) mit einem GC Modell 3400 der Firma Varian durchgeführt.

Drehwerte: Die Drehwerte wurden mit einem Polarimeter 241 der Firma Perkin-Elmer (Standardküvette, $1 \mathrm{dm}$ ) bestimmt. Die Werte für die spezifische Drehung $[\alpha]_{\lambda}^{\mathrm{T}}$ sind in der Einheit $\left[\operatorname{grad~cm}{ }^{3} \mathrm{dm}^{-1} \mathrm{~g}^{-1}\right]$ angegeben.

Elementaranalysen: Das Mikroanalytische Laboratorium des Instituts für Organische Chemie der Universität Göttingen führte die Elementaranalysen durch.

\subsection{Chromatographische Methoden}

Dünnschichtchromatographie: Es wurden DC-Fertigfolien Alugram SIL G/UV 254 der Firma Macherey \& Nagel (Schichtdicke $0.25 \mathrm{~mm}$ ) verwendet. Angegeben sind die $\mathrm{R}_{\mathrm{f}}$-Werte (Laufhöhe relativ zur Laufmittelfront) sowie die verwendeten Laufmittelgemische. Als Abkürzungen für verwendete Lösungsmittel werden benutzt: EE (Essigsäureethylester), PE (Petrolether des Siedebereichs 35 bis $60{ }^{\circ} \mathrm{C}$ ), $\mathrm{Et}_{2} \mathrm{O}$ (Diethylether), $\mathrm{CH}_{2} \mathrm{Cl}_{2}$ (Dichlormethan). Zur Detektion wurden neben UV-Licht der Wellenlänge $254 \mathrm{~nm}$ Anfärbereagenzien wie eine 1 proz. wässrige Kaliumpermanganatlösung oder ein Gemisch aus $0.5 \mathrm{~g}$ Vanillin, $3 \mathrm{ml}$ konzentrierter Schwefelsäure, $10 \mathrm{ml}$ konzentrierter Essigsäure in $85 \mathrm{ml}$ Methanol verwendet. 
Säulenchromatographie: Für chromatographische Reinigungen unter normalem Druck wurde Kieselgel 60 der Firma Merck mit einer Korngröße von 0.05 - 0.20 mm eingesetzt. Arbeiten unter 1.0 - 1.5 bar Druck (Flash-Chromatographie) ${ }^{[12]}$ wurden an Kieselgel 60 der Firma Merck mit einer Korngröße von 0.040 - $0.063 \mathrm{~mm}$ durchgeführt. Wenn in den Arbeitsvorschriften nicht anders angegeben, wurden für präparative Trennungen Substanz: Adsorbensverhältnisse zwischen $1: 100$ und $1: 200$ gewählt. Für Säulenfiltrationen wurden Verhältnisse von $1: 10$ bis $1: 20$ eingestellt.

Hochleistungsflüssigkeitschromatographie: Zur analytischen und präparativen Untersuchung und Aufreinigung von Substanzproben kam eine HPLC-Anlage der Firma Kontron mit Lösungsmittelpumpe 422, Mischkammer 425, UV-Detektor 322 und einem Probensammler Advantec SF-2120 der Firma Advantec zum Einsatz. Die Software zur Datenaufnahme und Datenauswertung DS450-MT2 stammt ebenfalls von der Firma Kontron. Analytische Untersuchungen wurden an einer LiChrospher Si 60 (4 x 125 mm) bzw. LiChrospher 100 RP-18 (4 x 125 mm) HPLC-Säule der Firma Merck, präparative Trennungen an einer Kontosorb 10 SIL (10 x 250 mm) Säule der Firma Kontron durchgeführt.

Es wurden Eluenten in HPLC-Qualität der Firmen Merck und Acros und bidestilliertes Wasser eingesetzt. Sowohl die Laufmittel als auch die zu analysierenden Proben wurden vor Gebrauch membranfiltriert $(0.2 \mu \mathrm{m})$.

Gaschromatographie: Gaschromatographische Untersuchungen wurden an einem GC Modell 3400 der Firma Varian oder einem GC Modell HRGC 5300 der Firma Carlo Erba durchgeführt. Die Bestimmung von Enantiomerenüberschüssen geschah an einer Kapillarsäule OV 1701 (25 m, 0.25 mm), die mit 20 \% Heptakis-(2,6-O-methyl-3-O-pentyl)$\beta$-cyclodextrin in Polysiloxan als chiraler stationärer Phase beladen war. Bei gewöhnlichen Untersuchungen gelangte eine SE 30 Säule $(25 \mathrm{~m}, 0.32 \mathrm{~mm})$ der Firma Chrompack zum Einsatz.

\subsection{Software}

Die Textverarbeitung erfolgte mit dem Programm Word für Windows 95 der Firma Microsoft. Die chemischen Formelbilder wurden mit dem Zeichenprogramm ChemDraw Ultra 5.0 der Firma CambridgeSoft erstellt. 


\section{Synthese von 2-Hydroxyphenazin (2)}

$2.1 \quad 1,2,4-$ Triacetoxybenzol $(44)^{[70]}$

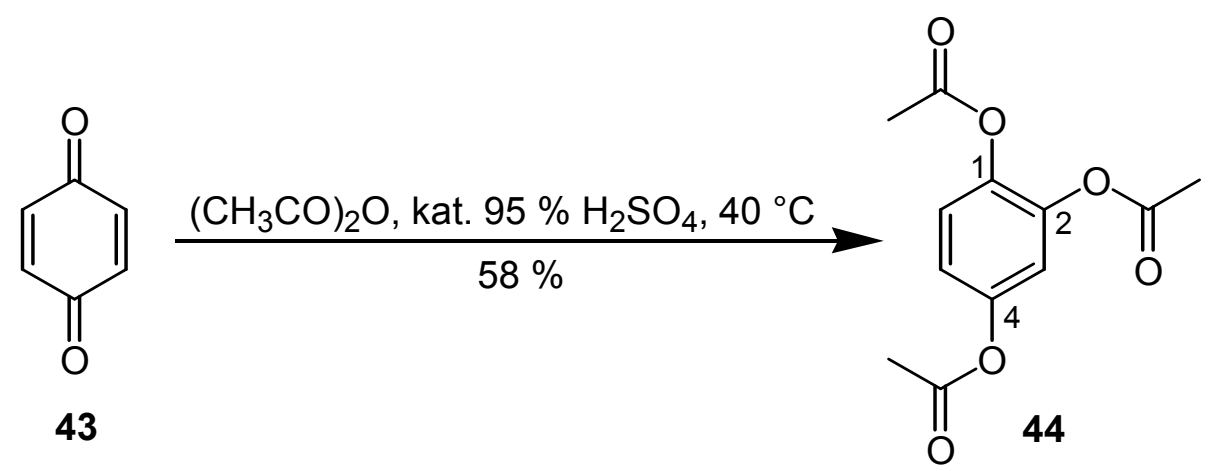

In einer Argon-Atmosphäre wurden zu einer Lösung von $110 \mathrm{ml}(119 \mathrm{~g}, 1.16$ mol, 3.7 Äquiv.) frisch destillierten Essigsäureanhydrids und $3.0 \mathrm{ml}$ (5.52 g, 56 mmol, 0.18 Äquiv.) konz. Schwefelsäure, 34.0 g (0.32 mol, 1.0 Äquiv.) 1,4-Benzochinon (43) so zugefügt, daß die Temperatur im Reaktionsgefäß zwischen 40 und $50{ }^{\circ} \mathrm{C}$ lag. Nach dem Ende der Wärmeentwicklung wurde die Reaktionslösung in $200 \mathrm{ml}$ Wasser eingetragen und ca. $10 \mathrm{~min}$. unter Eiskühlung gerührt, bis das Produkt auskristallisierte. Man saugte ab, wusch mit $30 \mathrm{ml}$ Wasser und kristallisierte aus Methanol um. Es wurden 45.9 g 1,2,4-Triacetoxybenzol (44) (58\% Ausbeute, Lit. ${ }^{[70]} 80 \%$ ) erhalten.

$\mathbf{R}_{\mathbf{f}}=0.37\left(\mathrm{Et}_{2} \mathrm{O} / \mathrm{PE}=5: 1\right)$.

Schmp.: $97^{\circ} \mathrm{C}$ (Lit. $\left.{ }^{[70]} 96.5-97^{\circ} \mathrm{C}\right)$.

IR (KBr): $\widetilde{v}=3054 \mathrm{~cm}^{-1}(=\mathrm{C}-\mathrm{H}), 1780,1762,(\mathrm{C}=\mathrm{O}), 1608(\mathrm{C}=\mathrm{C}), 1498,1428,1374\left(\mathrm{CH}_{3}\right)$, 1210, 1190, $1170(=\mathrm{C}-\mathrm{H}), 840(\mathrm{C}=\mathrm{C})$.

$\mathbf{U V}\left(\mathrm{CH}_{3} \mathrm{CN}\right): \lambda_{\max }(\lg \varepsilon)=200 \mathrm{~nm}(4.20), 264$ (2.83).

${ }^{1} \mathbf{H}$ NMR $\left(300 \mathrm{MHz}, \mathrm{CDCl}_{3}\right): \delta=2.26\left(\mathrm{~s}, 9 \mathrm{H}, 3 \times \mathrm{CH}_{3}\right), 6.98\left(\mathrm{dd},{ }^{3} J_{5 \mathrm{H}, 6 \mathrm{H}}=9.0 \mathrm{~Hz},{ }^{4} J_{5 \mathrm{H}, 3 \mathrm{H}}=\right.$ $2.5 \mathrm{~Hz}, 1 \mathrm{H}, 5-\mathrm{H})$, teilweise überlagert von $7.01\left(\mathrm{~d},{ }^{4} J_{3 \mathrm{H}, 5 \mathrm{H}}=2.5 \mathrm{~Hz}, 1 \mathrm{H}, 3-\mathrm{H}\right), 7.15\left(\mathrm{~d},{ }^{3} J_{6 \mathrm{H}, 5 \mathrm{H}}\right.$ $=9.0 \mathrm{~Hz}, 1 \mathrm{H}, 6-\mathrm{H})$.

${ }^{13} \mathbf{C}$ NMR $\left(50 \mathrm{MHz}, \mathrm{CDCl}_{3}\right): \delta=$ '+' $20.50\left(2 \times \mathrm{CH}_{3}\right)$, '+' $20.95\left(\mathrm{CH}_{3}\right)$, '+' 116.98, '+' 119.37, '+' 123.45 (C-3, C-5, C-6), '-' 139.50, '-' 142.12, '-' 148.00 (C-1, C-2, C-4), '-' 167.69 , `-'168.02, `-' $168.71(3 \times \mathrm{C}=\mathrm{O})$.

MS $(70 \mathrm{eV}): m / z(\%)=252(8)\left[\mathrm{M}^{+}\right], 210(17)\left[\mathrm{M}^{+}-\mathrm{C}_{2} \mathrm{H}_{2} \mathrm{O}\right], 168(36)\left[210^{+}-\mathrm{C}_{2} \mathrm{H}_{2} \mathrm{O}\right], 126$ (100) $\left[168^{+}-\mathrm{C}_{2} \mathrm{H}_{2} \mathrm{O}\right], 43(14)\left[\mathrm{C}_{2} \mathrm{H}_{3} \mathrm{O}^{+}\right]$.

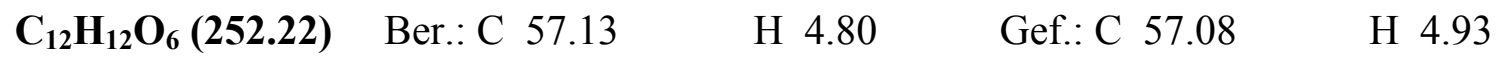




\section{$2.2 \quad 1,2,4-$ Trihydroxybenzol $(45)^{[70]}$}<smiles>CC(=O)Oc1ccc(OC(C)=O)c(OC(C)=O)c1</smiles>

$45.9 \mathrm{~g}$ (0.18 mol, 1.0 Äquiv.) 1,2,4-Triacetoxybenzol (44) wurden in einer Lösung von $10 \mathrm{ml}$ (11.8 g, 0.12 mol, 0.67 Äquiv.) konz. Salzsäure in $100 \mathrm{ml}$ Methanol während $1 \mathrm{~h}$ unter Sieden am Rückfluß zur Reaktion gebracht. Anschließend wurde die Reaktionsmischung i. Vak. am Rotationsverdampfer ohne Erhitzen bis zur Trockene eingedampft. Das erhaltene Rohprodukt wurde ohne weitere Aufreinigung für nachfolgende Reaktionen eingesetzt. Es entstanden $20.1 \mathrm{~g}$ des gewünschten Produkts. Die Ausbeute betrug $87 \%$ (Lit. ${ }^{[70]}>80 \%$ ). Analysenreines 1,2,4-Trihydroxybenzol (45) ließ sich durch Sublimation bei 0.01 Torr und $120{ }^{\circ} \mathrm{C}$ gewinnen.

$\mathbf{R}_{\mathbf{f}}=0.23(\mathrm{EE} / \mathrm{PE}=4: 1)$.

Schmp.: $140{ }^{\circ} \mathrm{C}\left(\right.$ Lit. $\left.^{[70]} 137-138^{\circ} \mathrm{C}\right)$.

IR (KBr): $\widetilde{v}=3202 \mathrm{~cm}^{-1}$ (OH--O), 3044 (=C-H), 1626 (C=C), 1554, 1518 (C-C), 1394, 1300 (=C-O), 1216, 1198, 1178, 1154 (=C-H), 840, $790(\mathrm{C}=\mathrm{C})$.

$\mathbf{U V}\left(\mathrm{CH}_{3} \mathrm{CN}\right): \lambda_{\max }(\lg \varepsilon)=195 \mathrm{~nm}(4.46), 290$ (3.52).

${ }^{1}$ H NMR (300 MHz, [D 6 ]DMSO): $\delta=6.02\left(\mathrm{dd},{ }^{3} J_{5 \mathrm{H}, 6 \mathrm{H}}=8.5 \mathrm{~Hz},{ }^{4} J_{5 \mathrm{H}, 3 \mathrm{H}}=2.5 \mathrm{~Hz}, 1 \mathrm{H}, 5-\mathrm{H}\right)$, $6.23\left(\mathrm{~d},{ }^{4} J_{3 \mathrm{H}, 5 \mathrm{H}}=2.5 \mathrm{~Hz}, 1 \mathrm{H}, 3-\mathrm{H}\right), 6.51\left(\mathrm{~d},{ }^{3} J_{6 \mathrm{H}, 5 \mathrm{H}}=8.5 \mathrm{~Hz}, 1 \mathrm{H}, 6-\mathrm{H}\right), 7.96(\mathrm{~s}, 1 \mathrm{H}, \mathrm{OH}$ $\left.\left[\mathrm{D}_{2} \mathrm{O}\right]\right), 8.44\left(\mathrm{~s}, 1 \mathrm{H}, \mathrm{OH},\left[\mathrm{D}_{2} \mathrm{O}\right]\right), 8.60\left(\mathrm{~s}, 1 \mathrm{H}, \mathrm{OH}\left[\mathrm{D}_{2} \mathrm{O}\right]\right)$.

${ }^{13}$ C NMR (50 MHz, [D $]$ DMSO): $\delta={ }^{+}+103.50$ (C-5 oder C-6), ' +' 105.16 (C-6 oder C-5), '+' $115.84(\mathrm{C}-3)$, '-' 137.51 (C-4), '-' 145.58 (C-1 oder C-2), '-' 150.16 (C-2 oder C-1).

MS $(70 \mathrm{eV}): m / z(\%)=126(100)\left[\mathrm{M}^{+}\right], 108(3)\left[\mathrm{M}^{+}-\mathrm{H}_{2} \mathrm{O}\right], 97(16)\left[\mathrm{M}^{+}-\mathrm{CHO}\right], 80$ (36) $\left[\mathrm{M}^{+}-\mathrm{CO}\right], 52(92)\left[\mathrm{C}_{4} \mathrm{H}_{4}^{+}\right], 42(8)\left[\mathrm{C}_{3} \mathrm{H}_{6}^{+}\right]$.

$\begin{array}{lll}\mathbf{C}_{6} \mathbf{H}_{6} \mathbf{O}_{3} \text { (126.11) } & \text { Ber.: C } 57.13 & \text { H } 4.80 \\ & \text { Gef.: C } 57.39 & \text { H } 4.90\end{array}$




\subsection{2-Hydroxyphenazin (2) nach Kehrmann und Cherpillod ${ }^{[68,71]}$}<smiles>Oc1ccc(O)c(O)c1</smiles>

45
1. $\mathrm{Ag}_{2} \mathrm{O}, \mathrm{Et}_{2} \mathrm{O}, \mathrm{RT}$, 2. o-Phenylendiamin (5), AcOH, RT $18 \%$

$50.0 \mathrm{~g}$ (0.35 mol, 4.28 Äquiv.) Natriumsulfat, für $12 \mathrm{~h}$ bei $150{ }^{\circ} \mathrm{C}$ getrocknet, wurden in $250 \mathrm{ml}$ trockenem Diethylether suspendiert. Anschließend wurden $10.0 \mathrm{~g}(80.0 \mathrm{mmol}, 1.0$ Äquiv.) 1,2,4-Trihydroxybenzol (45) in der Suspension gelöst und noch 40.4 g (170 mmol, 2.1 Äquiv.) Silber-(I)-oxid zugegeben. Das Reaktionsgemisch wurde $3 \mathrm{~h}$ bei Raumtemperatur gerührt, der graue Feststoff abfiltriert und mit $140 \mathrm{ml}$ Diethylether gewaschen. Das lichtempfindliche, gelbe Filtrat wurde in einem mit Aluminiumfolie verkleideten Rundkolben, i. Vak. am Rotationsverdampfer, ohne Erwärmen bis zur Trockene eingedampft. Das entstandene 2-Hydroxy-1,4-benzochinon wurde in $150 \mathrm{ml}$ Eisessig suspendiert (Ultraschall). In die Suspension wurden innerhalb von $10 \mathrm{~min} 10.0 \mathrm{~g}$ (92.5 mmol, 1.15 Äquiv.) $o$-Phenylendiamin (5) eingetragen und $20 \mathrm{~min}$ bei Raumtemperatur gerührt. Nachdem sich schwarze Kristalle gebildet hatten, fügte man noch $250 \mathrm{ml}$ Wasser zu und ließ die Reaktionsmischung $8 \mathrm{~h}$ bei $4{ }^{\circ} \mathrm{C}$ im Kühlschrank stehen. Dann wurde der Niederschlag abfiltriert, in 10 proz. Natriumhydroxidlösung gelöst und erneut filtriert. Das Filtrat wurde mit konz. Essigsäure auf pH $\leq 5$ angesäuert. Nach einer Stunde Kühlen mit Eiswasser wurde der entstandene Niederschlag über eine Nutsche filtriert und $5 \mathrm{~h}$ im Trockenschrank bei $150{ }^{\circ} \mathrm{C}$ getrocknet. Die erhaltenen 3.04 g Rohprodukt wurden auf $30 \mathrm{~g}$ Kieselgel aufgezogen und unter Druck an $250 \mathrm{~g}$ Kieselgel chromatographiert $\left(\mathrm{Et}_{2} \mathrm{O} / \mathrm{PE}=3: 1 \rightarrow \mathrm{Et}_{2} \mathrm{O}\right)$. Es wurden $2.86 \mathrm{~g}$ (Ausbeute $=$ 18 \%; Lit. ${ }^{[68]} 87$ \%) des gewünschten Produkts (2) als hellgelber Feststoff erhalten. 
2.4 2-Hydroxyphenazin (2) nach $O t t^{[69]}$<smiles>O=C1C=CC(=O)C=C1</smiles>

43<smiles>Nc1ccccc1N</smiles>

5

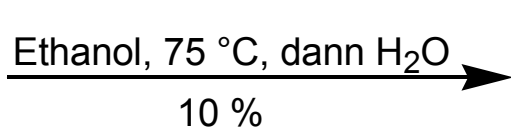
$10 \%$<smiles>Oc1ccc2nc3ccccc3nc2c1</smiles>

2

$500 \mathrm{mg}$ (4.6 mmol, 1.0 Äquiv.) 1,4-Benzochinon (43) wurden in $5 \mathrm{ml}$ trockenem Ethanol (Na) gelöst, indem die Suspension mit einem Fön leicht erhitzt wurde. Nach dem Abkühlen gab man $500 \mathrm{mg}$ (4.6 mmol, 1.0 Äquiv.) o-Phenylendiamin (5) zu. Die Lösung färbte sich dunkelrot und schwarzrote Kristalle schieden sich ab. Nach einer Stunde Stehen bei Raumtemperatur erhitzte man die Reaktionsmischung unter Rühren noch ca. 30 min bei $75{ }^{\circ} \mathrm{C}$, fügte nach dem Abkühlen ca. $1 \mathrm{ml}$ Wasser zu und stellte sie mehrere Stunden bei $4{ }^{\circ} \mathrm{C}$ in den Kühlschrank. Die kalte Reaktionsmischung wurde über eine Nutsche filtriert und die auf dem Filter verbliebenen braunschwarzen Kristalle in 0.5 M Natronlauge gelöst. Dann filtrierte man vom Unlöslichen ab und säuerte das Filtrat mit konz. Essigsäure an, wobei ein brauner Feststoff ausfiel. Dieser wurde filtriert, i. Vak. getrocknet und zur Aufreinigung an $50 \mathrm{~g}$ Kieselgel chromatographiert $\left(\mathrm{Et}_{2} \mathrm{O} / \mathrm{PE}=3: 1 \rightarrow \mathrm{Et}_{2} \mathrm{O}\right)$. Es wurden $100 \mathrm{mg}(10 \%$ Ausbeute Lit. $^{[69]}$ „etwa $90 \%$ \%) 2-Hydroxyphenazin (2) als hellgelber Feststoff isoliert.

$\mathbf{R}_{\mathbf{f}}=0.66(\mathrm{EE} / \mathrm{PE}=3: 1)$.

Schmp.: $250{ }^{\circ} \mathrm{C}$, Zers. (Lit. ${ }^{[69]} 253-254$, Zers.).

IR $(\mathrm{KBr}): \widetilde{v}=3420-2595 \mathrm{~cm}^{-1}(\mathrm{OH},=\mathrm{C}-\mathrm{H}), 1632,1602,1476,1452,1420(\mathrm{C}=\mathrm{C}), 1282$ (O-H), 1222, 1200 (C-O).

UV $\left(\mathrm{CH}_{3} \mathrm{CN}\right): \lambda_{\max }(\lg \varepsilon)=216 \mathrm{~nm}$ (4.36), 253 (4.87), 355 (3.94), 391 (3.83).

${ }^{1}$ H NMR (300 MHz, [D $\left.\left.\mathrm{D}_{6}\right] \mathrm{DMSO}\right): \delta=7.34\left(\mathrm{~d},{ }^{4} J_{1 \mathrm{H}, 3 \mathrm{H}}=2.5 \mathrm{~Hz}, 1 \mathrm{H}, 1-\mathrm{H}\right), 7.57\left(\mathrm{dd},{ }^{3} J_{3 \mathrm{H}, 4 \mathrm{H}}=\right.$ $\left.9.5 \mathrm{~Hz},{ }^{4} J_{3 \mathrm{H}, 1 \mathrm{H}}=2.5 \mathrm{~Hz}, 1 \mathrm{H}, 3-\mathrm{H}\right), 7.80\left(\mathrm{ddd},{ }^{3} J_{7 \mathrm{H}, 6 \mathrm{H}}=8.5 \mathrm{~Hz},{ }^{3} J_{7 \mathrm{H}, 8 \mathrm{H}}=6.5 \mathrm{~Hz},{ }^{4} J_{7 \mathrm{H}, 9 \mathrm{H}}=1.5\right.$ $\mathrm{Hz}$ bzw. ${ }^{3} J_{8 \mathrm{H}, 9 \mathrm{H}}=8.5 \mathrm{~Hz},{ }^{3} J_{8 \mathrm{H}, 7 \mathrm{H}}=6.5 \mathrm{~Hz},{ }^{4} J_{8 \mathrm{H}, 6 \mathrm{H}}=1.5 \mathrm{~Hz}, 1 \mathrm{H}, 7-\mathrm{H}$ oder 8-H), teilweise überlagert von $7.87\left(\mathrm{ddd},{ }^{3} J_{8 \mathrm{H}, 9 \mathrm{H}}=8.5 \mathrm{~Hz},{ }^{3} J_{8 \mathrm{H}, 7 \mathrm{H}}=6.5 \mathrm{~Hz},{ }^{4} J_{8 \mathrm{H}, 6 \mathrm{H}}=1.5 \mathrm{~Hz}\right.$ bzw. ${ }^{3} J_{7 \mathrm{H}, 6 \mathrm{H}}=8.5$ $\mathrm{Hz},{ }^{3} J_{7 \mathrm{H}, 8 \mathrm{H}}=6.5 \mathrm{~Hz},{ }^{4} J_{7 \mathrm{H}, 9 \mathrm{H}}=1.5 \mathrm{~Hz}, 1 \mathrm{H}, 8-\mathrm{H}$ oder $\left.7-\mathrm{H}\right), 8.10\left(\mathrm{~d},{ }^{3} J_{4 \mathrm{H}, 3 \mathrm{H}}=9.5 \mathrm{~Hz}, 1 \mathrm{H}, 4-\mathrm{H}\right)$, überlagert von $8.11\left(\mathrm{dd},{ }^{3} J_{6 \mathrm{H}, 7 \mathrm{H}}=8.5 \mathrm{~Hz},{ }^{4} J_{6 \mathrm{H}, 8 \mathrm{H}}=1.5 \mathrm{~Hz}\right.$ bzw. ${ }^{3} J_{9 \mathrm{H}, 8 \mathrm{H}}=8.5 \mathrm{~Hz},{ }^{4} J_{9 \mathrm{H}, 7 \mathrm{H}}=1.5$ $\mathrm{Hz}, 1 \mathrm{H}, 6-\mathrm{H}$ oder 9-H), überlagert von $8.15\left(\mathrm{dd},{ }^{3} J_{9 \mathrm{H}, 8 \mathrm{H}}=8.5 \mathrm{~Hz},{ }^{4} J_{9 \mathrm{H}, 7 \mathrm{H}}=1.5 \mathrm{~Hz}\right.$, bzw. ${ }^{3} J_{6 \mathrm{H}, 7 \mathrm{H}}=8.5 \mathrm{~Hz},{ }^{4} J_{6 \mathrm{H}, 8 \mathrm{H}}=1.5 \mathrm{~Hz}, 1 \mathrm{H}, 9-\mathrm{H}$ oder $\left.6-\mathrm{H}\right), 10.85$ (s br, $1 \mathrm{H}, \mathrm{OH}\left[\mathrm{D}_{2} \mathrm{O}\right]$ ). 


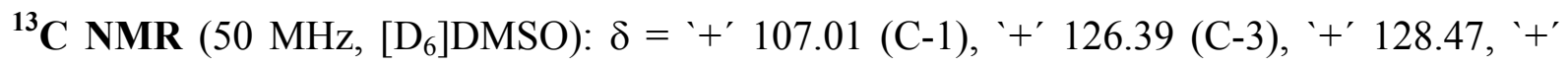
128.72, ' +129.27, '+' 130.45, '+' 130.70 (C-4, C-6, C-7, C-8, C-9), '-' 139.47, '-' 140.90, '-' 142.87, '-' 144.75 (C-4a, C-5a, C-9a, C-10a), ’-' 159.50 (C-2).

MS (70 eV): $m / z(\%)=196(100)\left[\mathrm{M}^{+}\right], 168(5)\left[\mathrm{M}^{+}-2 \mathrm{~N}\right], 140(3)\left[168^{+}-\mathrm{CO}\right], 114$ (2), 102 (2), 98 (4), 76 (3), 64 (4), 50 (4).

$\begin{array}{lll}\mathbf{C}_{12} \mathbf{H}_{8} \mathbf{N}_{\mathbf{2}} \mathrm{O} \text { (196.21) } & \text { Ber.: C } 73.45 & \text { H } 4.11 \\ & \text { Gef.: C } 73.38 & \text { H } 4.06\end{array}$




\section{$3 \quad$ Synthese von Phenazinether rac-51}

$3.1 \quad(3 R S)-( \pm)-1-M e t h y l s u l f o n y l-3,7-d i m e t h y l-n o n-6-e n(\text { rac-49) })^{[121]}$<smiles>CC(C)=CCCC(C)CCO</smiles>

rac-47

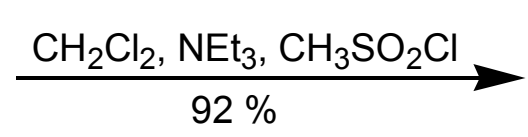

$92 \%$<smiles>[B]C(C)=CCCC(C)CCOS(C)(=O)=O</smiles>

rac-49

Es wurden bei $0^{\circ} \mathrm{C} 156 \mathrm{mg}$ (1.0 mmol, 1.0 Äquiv.) Citronellol (rac-47) in $3 \mathrm{ml}$ trockenem Dichlormethan $\left(\mathrm{P}_{4} \mathrm{O}_{10}\right)$ gelöst. Nacheinander wurden $111 \mathrm{mg}$ (1.1 mmol, 1.1 Äquiv.) Triethylamin und $126 \mathrm{mg}$ (1.1 mmol, 1.1 Äquiv.) Methansulfonsäurechlorid zugespritzt. Die Reaktionsmischung wurde auf Raumtemperatur erwärmt und nach ca. 15 min. Rühren war alles Substrat umgesetzt (DC Kontrolle: $\mathrm{Et}_{2} \mathrm{O} / \mathrm{PE}=1: 3$ ). Man überführte den Ansatz mit 5 $\mathrm{ml}$ Dichlormethan in einen Scheidetrichter und extrahierte zweimal mit je $5 \mathrm{ml}$ gesättigter Natriumhydrogencarbonatlösung und einmal mit $5 \mathrm{ml}$ gesättigter Natriumchloridlösung. Man trocknete über Natriumsulfat, filtrierte und entfernte das Lösungsmittel am Rotationsverdampfer i. Vak. Der verbliebene Rückstand wurde an 15 g Kieselgel unter Druck chromatographiert $\left(\mathrm{Et}_{2} \mathrm{O} / \mathrm{PE}=1: 1\right)$. Es wurden $210 \mathrm{mg}(92 \%$ Ausbeute $)$ des Produkts rac-49 als Ö1 isoliert.

$\mathbf{R}_{\mathbf{f}}=0.47\left(\mathrm{Et}_{2} \mathrm{O} / \mathrm{PE}=1: 1\right)$.

${ }^{1} \mathbf{H}$ NMR $\left(200 \mathrm{MHz}, \mathrm{CDCl}_{3}\right): \delta=0.92\left(\mathrm{~d},{ }^{3} \mathrm{~J}_{3 \mathrm{CH}_{3}, 3 \mathrm{H}}=6.5 \mathrm{~Hz}, 3 \mathrm{H}, 3-\mathrm{CH}_{3}\right), 1.08-1.46(\mathrm{~m}, 3 \mathrm{H}$, 3-H, 4- $\left.\mathrm{H}_{2}\right), 1.47-1.88\left(\mathrm{~m}, 2 \mathrm{H}, 2-\mathrm{H}_{2}\right)$ überlagert von $1.59\left(\mathrm{~s}, 3 \mathrm{H}, 7-\mathrm{CH}_{3}\right)$ und $1.67(\mathrm{~s}, 3 \mathrm{H}, 8-$ $\left.\mathrm{H}_{3}\right), 1.89-2.10\left(\mathrm{~m} 2 \mathrm{H}, 5-\mathrm{H}_{2}\right), 2.97\left(\mathrm{~s}, 3 \mathrm{H}, \mathrm{OSO}_{2} \mathrm{CH}_{3}\right), 4.26\left(\mathrm{t},{ }^{3} J_{1 \mathrm{H}_{2}, 2 \mathrm{H}_{2}}=6.5 \mathrm{~Hz}, 2 \mathrm{H}, 1-\mathrm{H}_{2}\right)$, $5.07(\mathrm{~m}, 1 \mathrm{H}, 6-\mathrm{H})$.

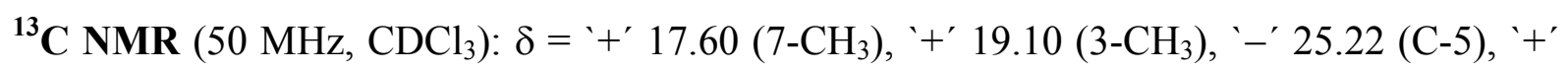
25.65 (C-8), '+' 28.91 (C-3), '-' 35.86 (C-4 oder C-2), '-' 36.74 (C-2 oder C-4), '+' 37.26 $\left(\mathrm{OSO}_{2} \mathrm{CH}_{3}\right)$, '-' $68.55(\mathrm{C}-1)$, '+' $124.22(\mathrm{C}-6)$, '-' $131.48(\mathrm{C}-7)$.

MS $(70 \mathrm{eV}): m / z(\%)=234(4)\left[\mathrm{M}^{+}\right], 138(42)\left[\mathrm{M}^{+}-\mathrm{HOSO}_{2} \mathrm{CH}_{3}\right], 123(16)\left[138^{+}-\mathrm{CH}_{3}\right], 109$ (16) $\left[138^{+}-\mathrm{C}_{2} \mathrm{H}_{5}\right], 95(46)\left[\mathrm{OSO}_{2} \mathrm{CH}_{3}{ }^{+}\right], 81(67)\left[138^{+}-\mathrm{C}_{4} \mathrm{H}_{9}\right], 69(75)\left[\mathrm{C}_{5} \mathrm{H}_{9}{ }^{+}\right]$.

$\mathrm{C}_{11} \mathrm{H}_{22} \mathrm{SO}_{3}$ (234.13) 


\section{$3.2 \quad(3 R S)-( \pm)-1-T o l u o l s u l f o n y l-3,7-d i m e t h y l-n o n-6-e n ~(r a c-48)^{[122]}$}<smiles>CC(C)=CCCC(C)CCO</smiles>

rac-47

\section{$\underset{85 \%}{\text { Pyridin, } \mathrm{CH}_{3} \mathrm{C}_{6} \mathrm{H}_{4} \mathrm{SO}_{2} \mathrm{Cl}, \mathrm{RT}}$}

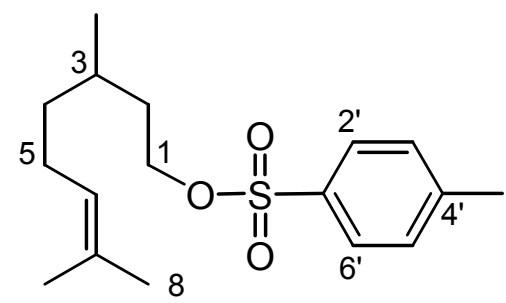

rac-48

In $5 \mathrm{ml}$ frisch destilliertem Pyridin (KOH) wurden bei $0{ }^{\circ} \mathrm{C} 312 \mathrm{mg}$ (2.0 mmol, 1.0 Äquiv.) Citronellol (rac-47) gelöst und anschließend $572 \mathrm{mg}$ (3.0 mmol, 1.5 Äquiv.) p-Toluolsulfonsäurechlorid zugegeben. Nach ca. $8 \mathrm{~h}$ bei $0{ }^{\circ} \mathrm{C}$ wurden $3 \mathrm{ml}$ Wasser zugegeben, einige Minuten gerührt und in $10 \mathrm{ml}$ Dichlormethan aufgenommen. Nach dem Abtrennen der wäßrigen Phase wurde die organische Phase zweimal mit je $5 \mathrm{ml} 2 M$ Salzsäure und je einmal mit $5 \mathrm{ml}$ gesättigter Natriumhydrogencarbonat- und $5 \mathrm{ml}$ gesättigter Natriumchloridlösung gewaschen. Nach dem Trocknen über Natriumsulfat und dem Evaporieren des Solvens erhielt man ein Öl, das an $20 \mathrm{~g}$ Kieselgel aufgereinigt wurde (Eluent: $\mathrm{Et}_{2} \mathrm{O} / \mathrm{PE}=1: 10$ ). Es wurden 504 mg (85\% Ausbeute) des Tosylats rac-48 erhalten.

$\mathbf{R}_{\mathbf{f}}=0.63\left(\mathrm{Et}_{2} \mathrm{O} / \mathrm{PE}=1: 2\right)$.

${ }^{1} \mathbf{H}$ NMR $\left(200 \mathrm{MHz}, \mathrm{CDCl}_{3}\right): \delta=0.82\left(\mathrm{~d},{ }^{3} \mathrm{~J}_{3 \mathrm{CH}_{3}, 3 \mathrm{H}}=6.5 \mathrm{~Hz}, 3 \mathrm{H}, 3-\mathrm{CH}_{3}\right), 1.00-1.80(\mathrm{~m}, 5 \mathrm{H}$, 2- $\left.\mathrm{H}_{2}, 3-\mathrm{H}, 4-\mathrm{H}_{2}\right)$ überlagert von $1.57\left(\mathrm{~s}, 3 \mathrm{H}, 7-\mathrm{CH}_{3}\right)$ und $1.68\left(\mathrm{~s}, 3 \mathrm{H}, 8-\mathrm{H}_{3}\right), 1.77-2.08(\mathrm{~m}$, $\left.2 \mathrm{H}, 5-\mathrm{H}_{2}\right), 2.44\left(\mathrm{~s}, 3 \mathrm{H}, 4^{\prime}-\mathrm{CH}_{3}\right), 4.06\left(\mathrm{t},{ }^{3} J_{\mathrm{H}_{2}, 2 \mathrm{H}_{2}}=6.0 \mathrm{~Hz}, 2 \mathrm{H}, 1-\mathrm{H}_{2}\right), 5.02\left(\mathrm{~m}_{\mathrm{C}}, 1 \mathrm{H}, 6-\mathrm{H}\right)$, $7.34\left(\mathrm{~d},{ }^{3} J_{3^{\prime} \mathrm{H}, 2^{\prime} \mathrm{H}}={ }^{3} J_{5^{\prime}}{ }^{\prime}, 6^{\prime} \mathrm{H}=8.0 \mathrm{~Hz}, 2 \mathrm{H}, 3^{\prime}-\mathrm{H}, 5^{\prime}-\mathrm{H}\right), 7.79\left(\mathrm{~d},{ }^{3} J_{2^{\prime}} \mathrm{H}, 3^{\prime} \mathrm{H}={ }^{3} J_{6^{\prime} \mathrm{H}, 5^{\prime} \mathrm{H}}=8.0 \mathrm{~Hz}, 2 \mathrm{H}\right.$, 2'-H, 6'-H).

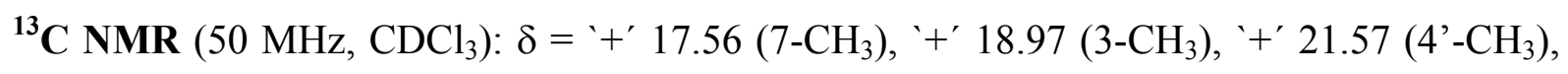
'-' $25.20(\mathrm{C}-5)$, '+' $25.64(\mathrm{C}-8)$, '+' 28.79 (C-3), '-' 35.59 (C-4 oder C-2), '-' 36.64 (C-2 oder C-4), ’-' 69.00 (C-1), '+' 124.25 (C-6), '+' 127.81 (C-3', C-5’), ’+' 129.75 (C-2', C-6’), '-' 131.48 (C-7), '-' 133.15 (C-4'), '-' 144.59 (C-1').

MS $\left(200 \mathrm{eV}, \mathrm{DCI} / \mathrm{NH}_{3}\right): m / z(\%)=638(30)\left[2 \mathrm{M}+\mathrm{NH}_{4}^{+}\right], 328(100)\left[\mathrm{M}+\mathrm{NH}_{4}^{+}\right]$.

$\mathrm{C}_{17} \mathrm{H}_{26} \mathrm{SO}_{3}(310.45)$

Ber.: C 65.77

H 8.44

Gef.: C 65.74

H 8.38 


\section{$3.3 \quad(3 R S)-( \pm)$ 1-Brom-3,7-dimethyl-6-hepten $(\text { rac-50 })^{[73]}$}<smiles>CC(C)=CCCC(C)CCO</smiles>

rac-47

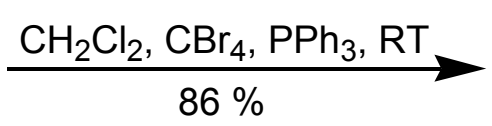

$\mathrm{Zu}$ einer gerührten Lösung von $8.81 \mathrm{~g}$ (50.0 mmol, 1.0 Äquiv.) Citronellol (rac-47) und $20.75 \mathrm{~g}$ (62.5 mmol, 1.25 Äquiv.) Tetrabromkohlenstoff in $80 \mathrm{ml}$ trockenem Dichlormethan $\left(\mathrm{P}_{4} \mathrm{O}_{10}\right)$ wurden bei $0{ }^{\circ} \mathrm{C} 19.6 \mathrm{~g}(75 \mathrm{mmol}, 1.50$ Äquiv.) Triphenylphosphin während $5 \mathrm{~min}$ hinzugefügt. Nach 15 min war die Reaktion laut dünnschichtchromatographischer Analyse der Reaktionsmischung beendet $\left(\mathrm{Et}_{2} \mathrm{O} / \mathrm{PE}=1: 1\right)$. Zur Aufarbeitung wurde das Lösungsmittel am Rotationsverdampfer i. Vak. entfernt und der Rückstand in $70 \mathrm{ml}$ Diethylether aufgenommen und filtriert. Der Feststoff wurde dann dreimal mit je $50 \mathrm{ml}$ Ether gewaschen, die organischen Phasen vereinigt und das Solvens i. Vak. entfernt. Der ölige Rückstand wurde im Kugelrohrofen $\left(10^{-2}\right.$ Torr $\left./ 100{ }^{\circ} \mathrm{C}\right)$ destilliert. Man erhielt $9.38 \mathrm{~g}$ (86\% Ausbeute) der Titelverbindung rac-50 als klares Öl.

$\mathbf{R}_{\mathbf{f}}=0.43\left(\mathrm{Et}_{2} \mathrm{O} / \mathrm{PE}=1: 2\right)$.

${ }^{1} \mathbf{H}$ NMR $\left(200 \mathrm{MHz}, \mathrm{CDCl}_{3}\right): \delta=0.88\left(\mathrm{~d},{ }^{3} \mathrm{~J}_{3 \mathrm{CH}_{3}, 3 \mathrm{H}}=6.5 \mathrm{~Hz}, 3 \mathrm{H}, 3-\mathrm{CH}_{3}\right), 1.05-1.45(\mathrm{~m}, 3 \mathrm{H}$, 3-H, 4- $\left.\mathrm{H}_{2}\right), 1.59\left(\mathrm{~s}, 3 \mathrm{H}, 7-\mathrm{CH}_{3}\right)$ und $1.67\left(\mathrm{~s}, 3 \mathrm{H}, 8-\mathrm{H}_{3}\right)$ überlagert von $1.55-2.05(\mathrm{~m}, 4 \mathrm{H}, 2-$ $\left.\mathrm{H}_{2}, 5-\mathrm{H}_{2}\right), 3.39\left(\mathrm{ddd},{ }^{2} J_{1 \mathrm{H}_{\mathrm{B}}, 1 \mathrm{H}_{\mathrm{A}}}=15.0 \mathrm{~Hz},{ }^{3} J_{1 \mathrm{H}_{\mathrm{B}}, 2 \mathrm{H}_{\mathrm{A}}}=10.0 \mathrm{~Hz},{ }^{3} J_{1 \mathrm{H}_{\mathrm{B}}, 2 \mathrm{H}_{\mathrm{B}}}=7.0 \mathrm{~Hz}, 1 \mathrm{H}, 1-\mathrm{H}_{\mathrm{B}}\right)$ teilweise überlagert von $3.43\left(\mathrm{ddd},{ }^{2} J_{1 \mathrm{H}_{\mathrm{A}}, 1 \mathrm{H}_{\mathrm{B}}}=15.0 \mathrm{~Hz},{ }^{3} J_{1 \mathrm{H}_{\mathrm{A}}, 2 \mathrm{H}_{\mathrm{A}}}=9.5 \mathrm{~Hz},{ }^{3} J_{1 \mathrm{H}_{\mathrm{A}}, 2 \mathrm{H}_{\mathrm{B}}}=5.0 \mathrm{~Hz}\right.$, $\left.1 \mathrm{H}, 1-\mathrm{H}_{\mathrm{A}}\right), 5.07(\mathrm{~m}, 1 \mathrm{H}, 6-\mathrm{H})$.

${ }^{13} \mathbf{C}$ NMR (50 MHz, $\left.\mathrm{CDCl}_{3}\right): \delta=$ '+' $17.65\left(7-\mathrm{CH}_{3}\right)$, '+' $18.84\left(3-\mathrm{CH}_{3}\right)$, '-' $25.32(\mathrm{C}-5)$, '+' 25.69 (C-8), ’+' 31.36 (C-3), ’-' 32.06 (C-4 oder C-2), '-' 36.55 (C-2 oder C-4), ’-' 39.99 (C-1), '+' $124.42(\mathrm{C}-6),{ }^{\prime}-' 131.46(\mathrm{C}-7)$.

\section{$\mathrm{C}_{10} \mathrm{H}_{19} \mathrm{Br}(\mathbf{2 1 9 . 6 0 )}$}




\section{4 (3'RS)-( \pm ) 2-(3,7-Dimethyl-oct-6-enyloxy)-phenazin (rac-51)}

\section{Variante A}

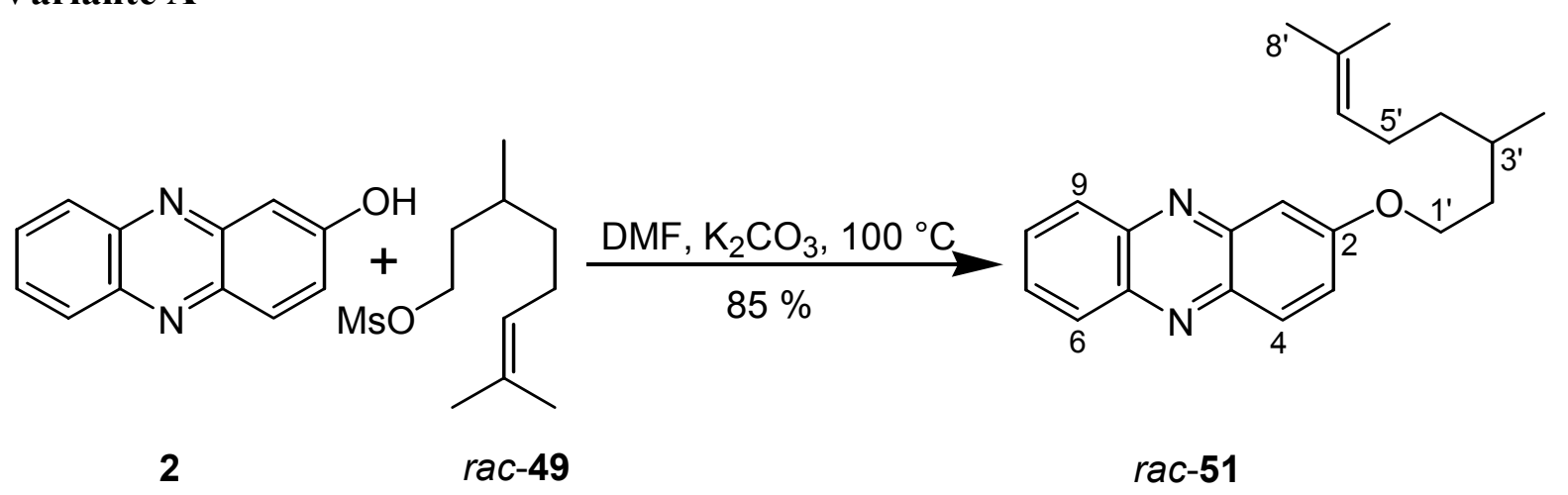

Es wurden 196 mg (1.0 mmol, 1.0 Äquiv.) 2-Hydroxyphenazin (2) bei Raumtemperatur in $5 \mathrm{ml}$ trockenem Dimethylformamid $\left(\mathrm{CaH}_{2}\right)$ gelöst und mit $691 \mathrm{mg}$ (5.0 mmol, 5.0 Äquiv.) wasserfreiem, fein gemörsertem Kaliumcarbonat versetzt und gerührt. Nach ca. 10 min spritzte man $220 \mathrm{mg}$ (0.94 mmol, 0.94 Äquiv.) des Mesylats rac-49 zu und erhitzte die Mischung auf $100{ }^{\circ} \mathrm{C}$ bis zur Beendigung des Umsatzes (DC-Kontrolle: $\mathrm{Et}_{2} \mathrm{O} / \mathrm{PE}=1: 1$ ). Nach dem Abkühlen verdünnte man mit $20 \mathrm{ml}$ Dichlormethan und schüttelte einmal mit $10 \mathrm{ml}$ Wasser aus. Die wässrige Phase wurde abgetrennt, mit Natriumchlorid gesättigt und zweimal mit je $10 \mathrm{ml}$ Dichlormethan extrahiert. Abschließend wurden die organischen Phasen vereinigt und zweimal mit je $10 \mathrm{ml}$ gesättigter Ammoniumchloridlösung gewaschen, über Natriumsulfat getrocknet und filtriert. Das Entfernen des Solvens am Rotationsverdampfer i. Vak. ergab das Rohprodukt, das an 40 g Kieselgel unter Druck chromatographiert wurde. Es wurden 284 mg (85 \% Ausbeute ) Produkt rac-51 als gelbes Öl isoliert.

\section{Variante B}

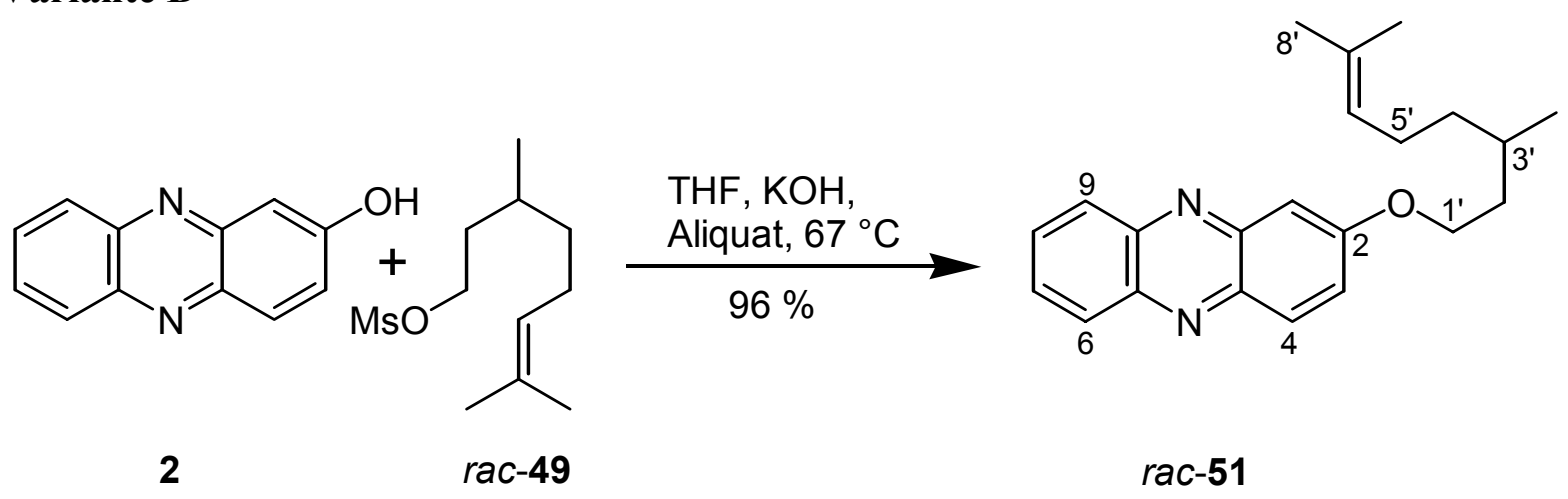

Man mischte $19.6 \mathrm{mg}$ (0.1 mmol, 1.0 Äquiv.) 2-Hydroxyphenazin (2) mit $5.6 \mathrm{mg}$ (0.1 mmol, 1.0 Äquiv.) fein gemörsertem Kaliumhydroxid und 6 mg Aliquat 336. Nach 10 min Rühren 
bei Raumtemperatur fügte man $23.4 \mathrm{mg}$ Mesylat rac-49 als Lösung in $2 \mathrm{ml}$ Tetrahydrofuran hinzu und erhitzte unter Sieden am Rückfluß. Nach etwa $10 \mathrm{~h}$ war die Reaktion beendet (DC Kontrolle: $\mathrm{Et}_{2} \mathrm{O} / \mathrm{PE}=1$ : 1), die Reaktionsmischung wurde in $10 \mathrm{ml}$ Diethylether aufgenommen und zweimal mit je $5 \mathrm{ml}$ Wasser gewaschen. Die organische Phase wurde über Natriumsulfat getrocknet, filtriert und das Solvens entfernt. Das Rohprodukt wurde auf 200 mg Kieselgel aufgezogen und an $4 \mathrm{~g}$ Kieselgel chromatographisch gereinigt $\left(\mathrm{Et}_{2} \mathrm{O} / \mathrm{PE}=1: 3\right)$. Es wurden 32 mg des Produkts rac-51 (96 \% Ausbeute) als gelbes Öl isoliert.

$\mathbf{R}_{\mathbf{f}}=0.45\left(\mathrm{Et}_{2} \mathrm{O} / \mathrm{PE}=1: 2\right)$.

IR (KBr): $\widetilde{v}=3060,3028 \mathrm{~cm}^{-1}(=\mathrm{C}-\mathrm{H}), 2959,2925,2872,2855\left(\mathrm{CH}_{2}, \mathrm{CH}_{3}\right), 1632,1605$, 1560, $1518(\mathrm{C}=\mathrm{C}), 1483,1473,1445,1390,1380,\left(\mathrm{CH}_{2}, \mathrm{CH}_{3}\right), 1289,1197(\mathrm{C}-\mathrm{O}), 828,757$ ( $\mathrm{C}=\mathrm{C}$, aromat.).

UV (MeOH): $\lambda_{\max }(\lg \varepsilon) 216 \mathrm{~nm}=$ (3.40), 256 (3.91), 359 (2.97), 391 (2.90).

${ }^{1} \mathbf{H}$ NMR $\left(200 \mathrm{MHz}, \mathrm{CDCl}_{3}\right): \delta=1.00\left(\mathrm{~d},{ }^{3} J_{3 \mathrm{CH}_{3}, 3 \mathrm{H}}=6.0 \mathrm{~Hz}, 3 \mathrm{H}, 3{ }^{2}-\mathrm{CH}_{3}\right), 1.15-1.55(\mathrm{~m}, 3 \mathrm{H}$, 3'- $\left.-\mathrm{H}, 4^{\prime}-\mathrm{H}_{2}\right), 1.61\left(\mathrm{~s}, 3 \mathrm{H}, 7^{\prime}-\mathrm{CH}_{3}\right)$ und $1.68\left(\mathrm{~s}, 3 \mathrm{H}, 8^{\prime}-\mathrm{H}_{3}\right)$ überlagert von $1.56-2.15(\mathrm{~m}, 4 \mathrm{H}$, $\left.2^{\prime}-\mathrm{H}_{2}, 5^{\prime}-\mathrm{H}_{2}\right), 4.21\left(\mathrm{t},{ }^{3} J_{1^{\prime} \mathrm{H}_{2}, 2^{\prime} \mathrm{H}_{\mathrm{A}}}={ }^{3} J_{1}{ }^{\prime} \mathrm{H}_{2}, 2^{\prime} \mathrm{H}_{\mathrm{B}}=6.0 \mathrm{~Hz}, 2 \mathrm{H}, 1^{\prime}-\mathrm{H}_{2}\right), 5.11\left(\mathrm{t},{ }^{3} J_{6}{ }^{\prime} \mathrm{H}_{,}{ }^{\prime} \mathrm{H}_{\mathrm{A}}={ }^{3} J_{6}{ }^{\prime} \mathrm{H}, 5^{\prime} \mathrm{H}_{\mathrm{B}}\right.$ $\left.=7.0 \mathrm{~Hz}, 1 \mathrm{H}, 6{ }^{\prime}-\mathrm{H}\right), 7.40\left(\mathrm{~d},{ }^{4} J_{1 \mathrm{H}, 3 \mathrm{H}}=3.0 \mathrm{~Hz}, 1 \mathrm{H}, 1-\mathrm{H}\right), 7.49\left(\mathrm{dd},{ }^{3} J_{3 \mathrm{H}, 4 \mathrm{H}}=10.0 \mathrm{~Hz},{ }^{4} J_{3 \mathrm{H}, 1 \mathrm{H}}=\right.$ $3.0 \mathrm{~Hz}, 1 \mathrm{H}, 3-\mathrm{H}), 7.78\left(\mathrm{~m}_{\mathrm{C}}, 2 \mathrm{H}, 7-\mathrm{H}, 8-\mathrm{H}\right), 8.09\left(\mathrm{~d},{ }^{3} J_{4 \mathrm{H}, 3 \mathrm{H}}=10.0 \mathrm{~Hz}, 1 \mathrm{H}, 4-\mathrm{H}\right), 8.16-8.30$ (m, 2H, 6-H, 9-H).

${ }^{13} \mathbf{C}$ NMR (50 MHz, $\left.\mathrm{CDCl}_{3}\right): \delta=$ '+' $17.65\left(7^{\prime}-\mathrm{CH}_{3}\right)$, '+' $19.49\left(3^{\prime}-\mathrm{CH}_{3}\right)$, '-' $25.38(\mathrm{C}-5$ '), ' +' $25.69\left(\mathrm{C}-8^{\prime}\right)$, , '+' $29.56\left(\mathrm{C}-3^{\prime}\right)$, '-' 35.66 (C-4' oder C-2'), '-' 37.03 (C-2' oder C-4'), '-'

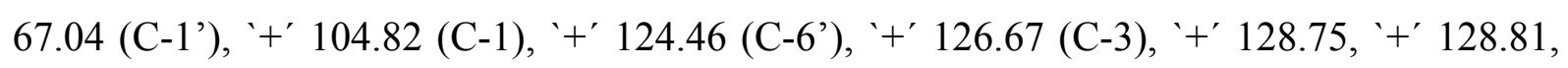
'+' 129.53, '+' 130.26 (C-6, C-7, C-8, C-9), '+' 130.38 (C-4), '-' 131.32 (C-7'), '-' 140.60, '-' 141.73, '-' 143.19, ’-' 145.03 (C-4a, C-5a, C-9a, C-10a), '-' 160.63 (C-2).

MS (70 eV): $m / z(\%)=334(86)\left[\mathrm{M}^{+}\right], 265(5)\left[\mathrm{M}^{+}-\mathrm{C}_{5} \mathrm{H}_{9}\right], 251$ (5) $\left[\mathrm{M}^{+}-\mathrm{C}_{6} \mathrm{H}_{11}\right], 209$ (8) $\left[\mathrm{C}_{13} \mathrm{H}_{9} \mathrm{~N}_{2} \mathrm{O}^{+}\right], 196(100)\left[\mathrm{C}_{12} \mathrm{H}_{8} \mathrm{~N}_{2} \mathrm{O}^{+}\right], 168$ (5) [196-CO], 69 (24) $\left[\mathrm{C}_{5} \mathrm{H}_{9}^{+}\right]$.

$\mathbf{C}_{22} \mathbf{H}_{26} \mathbf{N}_{2} \mathbf{O}$ (334.21) Ber.: C $78.99 \quad$ H 7.84

Gef.: C 79.19 H 8.03 


\section{$4 \quad$ Synthese des $\mathrm{C}_{6}$-Bausteins rac-6}

\section{1 (3RS)-( \pm )-5-tert.-Butyldimethylsilyoxy-3-methyl-pentan-1-ol $\left(\right.$ rac-52) ${ }^{[75]}$}

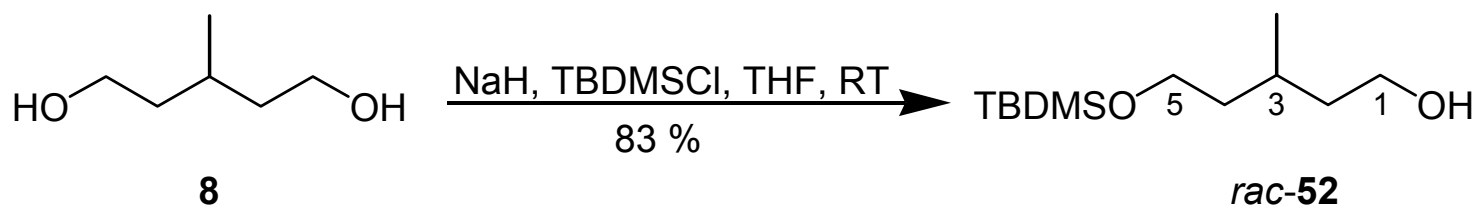

Durch Waschen mit Hexan wurden 3.35 g 60 proz. Natriumhydrid (2.02 g, 84 mmol, 1.0 Äquiv.) vom Mineralöl befreit und in $110 \mathrm{ml}$ Tetrahydrofuran (K) suspendiert. Durch ein Septum wurden $10.20 \mathrm{ml}$ (9.93 g, 84 mmol, 1.0 Äquiv.) 3-Methyl-1,5-pentandiol (8) zugespritzt und 60 min bei Raumtemperatur gerührt, währenddessen sich große Mengen eines weißen Niederschlags bildeten. Nun gab man $12.66 \mathrm{~g}$ (84 mmol, 1.0 Äquiv.) tert.Butyldimethylchlorsilan zur Reaktionsmischung und rührte kräftig für weitere 60 min bei Raumtemperatur. Der Ansatz wurde anschließend in $300 \mathrm{ml}$ Diethylether aufgenommen und je einmal mit $300 \mathrm{ml}$ gesättigter Natriumhydrogencarbonatlösung und $200 \mathrm{ml}$ gesättigter Natriumchloridlösung gewaschen, über Natriumsulfat getrocknet, filtriert und das Lösungsmittel i. Vak. am Rotationsverdampfer entfernt. Zur Reinigung wurde das Rohprodukt an 500 g Kieselgel chromatographiert $\left(\mathrm{Et}_{2} \mathrm{O} / \mathrm{PE}=1: 10 \rightarrow 1: 2\right)$. Es wurden $16.20 \mathrm{~g}$ (83\% Ausbeute) der Titelverbindung rac-52 als klares Öl isoliert.

$\mathbf{R}_{\mathbf{f}}=0.79\left(\mathrm{Et}_{2} \mathrm{O} / \mathrm{PE}=1: 1\right)$.

${ }^{1} \mathbf{H}$ NMR $\left(200 \mathrm{MHz}, \mathrm{C}_{6} \mathrm{D}_{6}\right): \delta=0.17\left[\mathrm{~s}, 6 \mathrm{H}, \mathrm{Si}\left(\mathrm{CH}_{3}\right)_{2}\right], 0.93\left(\mathrm{~d},{ }^{3} J_{\mathrm{CH}_{3}, \mathrm{CH}}=6.5 \mathrm{~Hz}, 3 \mathrm{H}, \mathrm{CH}_{3}\right)$, 0.97 (s br, 1H, OH), 1.08 [s, 9H, $\left.\mathrm{SiC}\left(\mathrm{CH}_{3}\right)_{3}\right], 1.18$ - 1.95 (m, 5H, 2- $\left.\mathrm{H}_{2}, 3-\mathrm{H}, 4 \mathrm{H}_{2}\right), 3.42\left(\mathrm{~m}_{\mathrm{C}}\right.$, $\left.2 \mathrm{H}, 1-\mathrm{H}_{2}\right) 3.57\left(\mathrm{~m}_{\mathrm{C}}, 2 \mathrm{H}, 5-\mathrm{H}_{2}\right)$.

${ }^{13} \mathbf{C}$ NMR $\left(50 \mathrm{MHz}, \mathrm{C}_{6} \mathrm{D}_{6}\right): \delta={ }^{+}{ }^{\prime}-5.17$ und ' $+{ }^{\prime}-5.14\left[\mathrm{Si}\left(\mathrm{CH}_{3}\right)_{3}\right]$, '-' $18.51\left[\mathrm{Si}-\mathrm{C}\left(\mathrm{CH}_{3}\right)_{3}\right]$, '+' $19.99\left(\mathrm{CH}_{3}\right)$, '+' $26.17\left[\mathrm{Si}-\mathrm{C}\left(\mathrm{CH}_{3}\right)_{3}\right]$, '+' $26.67(\mathrm{CH})$, '-' $40.22(\mathrm{C}-2$ und $\mathrm{C}-4)$, '-' 60.73 (C-5), '-'61.42 (C-1).

MS $\left(200 \mathrm{eV}, \mathrm{DCI} / \mathrm{NH}_{3}\right): m / z(\%)=250(100)\left[\mathrm{M}+\mathrm{NH}_{4}{ }^{+}\right], 233(92)\left[\mathrm{M}+\mathrm{H}^{+}\right]$.

$\mathrm{C}_{12} \mathrm{H}_{28} \mathrm{SiO}_{2}(232.44)$ 


\section{$4.2(3 R S)-( \pm)-t e r t .-B u t y l-(5-i o d o-3-m e t h y l-p e n t y l o x y)-d i m e t h y l s i l a n ~(r a c-6)$}

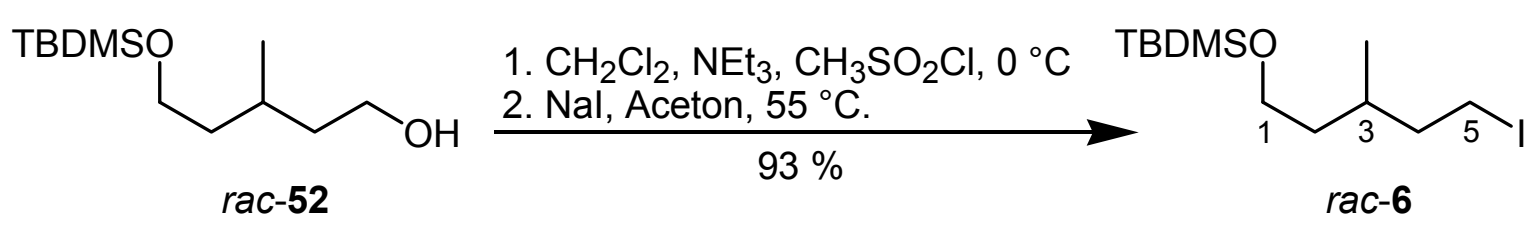

Man löste in $25 \mathrm{ml}$ Dichlormethan $1.16 \mathrm{~g}$ (5 mmol, 1.0 Äquiv.) des Alkohols rac-52 sowie $5 \mathrm{ml}$ (3.36 g, $36 \mathrm{mmol}, 7.2$ Äquiv.) frisch destilliertes Triethylamin und kühlte auf $0{ }^{\circ} \mathrm{C}$ ab. Nach Zugabe von $630 \mathrm{mg}$ (5.5 mmol, 1.1 Äquiv.) Methansulfonsäurechlorid rührte man noch ca. $2 \mathrm{~h}$ bei $0{ }^{\circ} \mathrm{C}$, bis das Substrat vollständig umgesetzt war (DC Kontrolle: $\mathrm{Et}_{2} \mathrm{O} / \mathrm{PE}=2: 1$ ). Zur Aufarbeitung gab man die Reaktionsmischung in $25 \mathrm{ml}$ gesättigte Natriumhydrogencarbonatlösung, trennte die organische Phase ab und extrahierte die wäßrige Phase noch dreimal mit je $20 \mathrm{ml}$ Dichlormethan. Die organischen Phasen wurden vereinigt, über Natriumsulfat getrocknet, filtriert und das Solvens i. Vak. entfernt. Das rohe Mesylat wurde in $40 \mathrm{ml}$ Aceton $\left(\mathrm{K}_{2} \mathrm{CO}_{3}\right)$ gelöst, dann fügte man $1.05 \mathrm{~g}$ (7.0 mmol, 1.4 Äquiv.) Natriumiodid zu und erhitzte unter Sieden zum Rückfluß. Nach etwa $4 \mathrm{~h}$ war die Reaktion beendet (DC Kontrolle: $\mathrm{Et}_{2} \mathrm{O} / \mathrm{PE}=1: 2$ ) und die abgekühlte Lösung wurde in $30 \mathrm{ml}$ Wasser aufgenommen. Die Reaktionsmischung wurde nun viermal mit je $30 \mathrm{ml}$ Diethylether extrahiert und die vereinigen organischen Phasen über Natriumsulfat getrocknet. Abfiltrieren vom Trockenmittel und Evaporieren des Lösungsmittels i. Vak. ergaben das Rohprodukt, das an $150 \mathrm{~g}$ Kieselgel chromatographisch gereinigt wurde $\left(\mathrm{Et}_{2} \mathrm{O} / \mathrm{PE}=1: 5\right)$. Es wurden $1.60 \mathrm{~g}$ des Iodids rac-6 (93\% Ausbeute) als klares Öl isoliert.

$\mathbf{R}_{\mathbf{f}}=0.66\left(\mathrm{Et}_{2} \mathrm{O} / \mathrm{PE}=1: 2\right)$.

IR (Film): $\widetilde{v}=2955,2928,2885,2857 \mathrm{~cm}^{-1}(\mathrm{C}-\mathrm{H}), 1471,1462,\left(\mathrm{CH}_{2}, \mathrm{CH}_{3}\right), 1255$ (C-OSi), 1098, 836 ( $\mathrm{Si}-\mathrm{OC}), 775\left(\mathrm{OSi}_{-} \mathrm{CH}_{3}\right), 740\left(\mathrm{C}-\mathrm{SiCH}_{3}\right)$.

$\mathbf{U V}\left(\mathrm{CH}_{3} \mathrm{CN}\right): \lambda_{\max }(\lg \varepsilon)=193 \mathrm{~nm}(3.84), 253$ (2.72).

${ }^{1} \mathbf{H}$ NMR $\left(300 \mathrm{MHz}, \mathrm{CDCl}_{3}\right): \delta=0.03\left[\mathrm{~s}, 6 \mathrm{H}, \mathrm{Si}\left(\mathrm{CH}_{3}\right)_{2}\right], 0.87$ [s, 9H, $\left.\mathrm{SiC}\left(\mathrm{CH}_{3}\right)_{3}\right]$, überlagert von $0.87\left(\mathrm{~d},{ }^{3} J_{3 \mathrm{CH}_{3}, 3 \mathrm{H}}=6.0 \mathrm{~Hz}, 3 \mathrm{H}, \mathrm{CH}_{3}\right), 1.34\left(\mathrm{~m}_{\mathrm{C}}, 1 \mathrm{H}, \mathrm{CH}\right), 1.46-1.60\left(\mathrm{~m}, 1 \mathrm{H}, 2-\mathrm{H}_{\mathrm{A}}\right.$ bzw. 4- $\left.\mathrm{H}_{\mathrm{A}}\right), 1.60-1.66\left(\mathrm{~m}, 2 \mathrm{H}, 2-\mathrm{H}_{\mathrm{B}}, 4-\mathrm{H}_{\mathrm{B}}\right), 1.80-1.97\left(\mathrm{~m}, 1 \mathrm{H}, 4-\mathrm{H}_{\mathrm{A}}\right.$ bzw. 2- $\left.\mathrm{H}_{\mathrm{A}}\right), 3.09-3.28(\mathrm{~m}$, $\left.2 \mathrm{H}, 1-\mathrm{H}_{2}\right), 3.63\left(\mathrm{~m}_{\mathrm{C}}, 2 \mathrm{H}, 5-\mathrm{H}_{2}\right)$. 


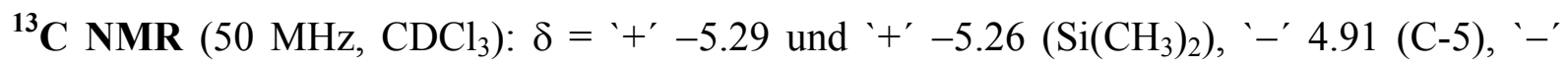

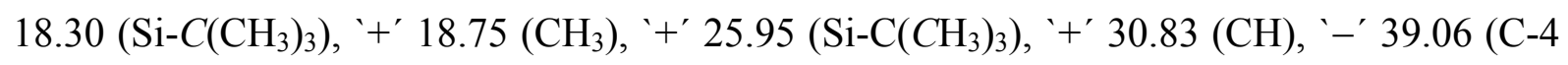
oder C-2), '-' 41.03 (C-2 oder C-4), '-' 60.92 (C-1).

MS $\left(200 \mathrm{eV}, \mathrm{DCI} / \mathrm{NH}_{3}\right): m / z(\%)=377(12)\left[\mathrm{M}+\mathrm{NH}_{3}+\mathrm{NH}_{4}{ }^{+}\right], 360(100)\left[\mathrm{M}+\mathrm{NH}_{4}{ }^{+}\right], 343$ (18) $\left[\mathrm{M}+\mathrm{H}^{+}\right]$.

$\mathbf{C}_{\mathbf{1 2}} \mathrm{H}_{27}$ SiOI (342.09) $\quad$ Ber.: C $42.09 \quad$ H 7.95

Gef.: C 42.38 H 7.76

\section{3 (3RS)-( \pm )-(5-Bromo-3-methyl-pentyloxy)-tert.-butyl-dimethylsilan (rac-53)}

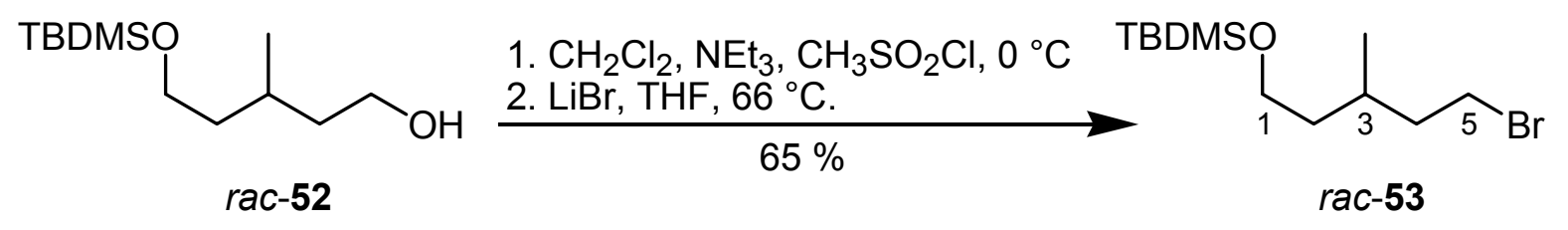

Man löste in $5 \mathrm{ml}$ Dichlormethan $232 \mathrm{mg}$ (1.0 mmol, 1.0 Äquiv.) des Alkohols rac-52, sowie $1.0 \mathrm{ml}$ (672 mg, $6.2 \mathrm{mmol}, 6.2$ Äquiv.) frisch destilliertes Triethylamin und kühlte auf $0{ }^{\circ} \mathrm{C}$ ab. Nach Zugabe von 126 mg (1.1 mmol, 1.1 Äquiv.) Methansulfonsäurechlorid rührte man noch etwa $1 \mathrm{~h}$ bei $0{ }^{\circ} \mathrm{C}$, bis das Substrat vollständig umgesetzt war (DC Kontrolle: $\mathrm{Et}_{2} \mathrm{O} / \mathrm{PE}=$ 2 : 1). Zur Aufarbeitung gab man die Reaktionsmischung in $5 \mathrm{ml}$ Wasser, trennte die organische Phase $a b$ und extrahierte die wäßrige Phase noch dreimal mit je $5 \mathrm{ml}$ Dichlormethan. Die organischen Phasen wurden vereinigt, über Natriumsulfat getrocknet, filtriert und das Solvens i. Vak. am Rotationsverdampfer entfernt. Das rohe Mesylat wurde dann in $10 \mathrm{ml}$ Tetrahydrofuran (K) gelöst, mit $120 \mathrm{mg}$ (1.38 mmol, 1.38 Äquiv.) Lithiumbromid versetzt und unter Sieden am Rückfluß zur Reaktion gebracht. Nach ca. $6 \mathrm{~h}$ war das Mesylat umgesetzt (DC Kontrolle: $\mathrm{Et}_{2} \mathrm{O} / \mathrm{PE}=1: 2$ ) und die abgekühlte Lösung wurde in $5 \mathrm{ml}$ Wasser aufgenommen. Die Reaktionsmischung wurde nun viermal mit je $10 \mathrm{ml}$ Dichlormethan extrahiert und die vereinigten organischen Phasen über Natriumsulfat getrocknet. Abfiltrieren vom Trockenmittel und Evaporieren des Lösungsmittels i. Vak. ergaben das Rohprodukt, das an $30 \mathrm{~g}$ Kieselgel unter Druck chromatographisch gereinigt wurde $\left(\mathrm{Et}_{2} \mathrm{O} / \mathrm{PE}\right.$ $=1: 5$ ). Es wurden 208 mg des Bromids rac-53 (65\% Ausbeute) als klares Öl isoliert. 
$\mathbf{R}_{\mathbf{f}}=0.78\left(\mathrm{Et}_{2} \mathrm{O} / \mathrm{PE}=1: 2\right)$.

IR (Film): $\widetilde{v}=2956,2929,2895,2857 \mathrm{~cm}^{-1}(\mathrm{C}-\mathrm{H}), 1471,1463,\left(\mathrm{CH}_{2}, \mathrm{CH}_{3}\right), 1255$ (C-OSi), 1098, 836 (Si-OC), $775\left(\mathrm{OSi}_{-} \mathrm{CH}_{3}\right), 740\left(\mathrm{C}-\mathrm{SiCH}_{3}\right), 647(\mathrm{C}-\mathrm{Br})$.

UV $\left(\mathrm{CH}_{3} \mathrm{CN}\right)$ : Die Verbindung rac-53 zeigt im Wellenlängenbereich $\lambda=195$ bis $400 \mathrm{~nm}$ keine UV-Absorption.

${ }^{1}$ H NMR $\left(300 \mathrm{MHz}, \mathrm{CDCl}_{3}\right): \delta=0.03\left[\mathrm{~s}, 6 \mathrm{H}, \mathrm{Si}\left(\mathrm{CH}_{3}\right)_{2}\right], 0.87\left[\mathrm{~s}, 9 \mathrm{H}, \mathrm{SiC}\left(\mathrm{CH}_{3}\right)_{3}\right]$, überlagert von $0.89\left(\mathrm{~d},{ }^{3} \mathrm{~J}_{3 \mathrm{CH}_{3}, 3 \mathrm{H}}=6.5 \mathrm{~Hz}, 3 \mathrm{H}, \mathrm{CH}_{3}\right), 1.20-2.00\left(\mathrm{~m}, 5 \mathrm{H}, 2-\mathrm{H}_{2}, 3-\mathrm{H}, 4-\mathrm{H}_{2}\right), 3.42\left(\mathrm{~m}_{\mathrm{C}}, 2 \mathrm{H}\right.$, $\left.5-\mathrm{H}_{2}\right), 3.63\left(\mathrm{~m}_{\mathrm{C}}, 2 \mathrm{H}, 1-\mathrm{H}_{2}\right)$.

${ }^{13} \mathbf{C}$ NMR $\left(50 \mathrm{MHz}, \mathrm{CDCl}_{3}\right): \delta={ }^{+}+{ }^{\prime}-5.30$ und $+{ }^{\prime}-5.27\left[\mathrm{Si}\left(\mathrm{CH}_{3}\right)_{2}\right]$, '-' $18.31\left[\mathrm{Si}-\mathrm{C}\left(\mathrm{CH}_{3}\right)_{3}\right]$, '+' $19.01\left(\mathrm{CH}_{3}\right)$, '+' $25.96\left[\mathrm{Si}-\mathrm{C}\left(\mathrm{CH}_{3}\right)_{3}\right]$, ’+' $28.63(\mathrm{CH})$, ’-' $31.90(\mathrm{C}-5)$, ’-' $39.29(\mathrm{C}-4$ oder C-2), '-' 40.10 (C-2 oder C-4), '-' 60.95 (C-1).

MS $(70 \mathrm{eV}): m / z(\%)=295(22)\left[\mathrm{M}^{+}\right], 239(2)\left[\mathrm{M}^{+}-\mathrm{C}_{4} \mathrm{H}_{8}\right], 167$ (10) $\left[\mathrm{C}_{6} \mathrm{H}_{14} \mathrm{Br}^{+}\right], 139$ (22) $\left[\mathrm{C}_{4} \mathrm{H}_{10} \mathrm{Br}^{+}\right], 115(10)\left[\mathrm{C}_{6} \mathrm{H}_{15} \mathrm{Si}\right], 73(56)\left[\mathrm{C}_{4} \mathrm{H}_{9} \mathrm{O}^{+}\right], 55(100)\left[\mathrm{C}_{4} \mathrm{H}_{7}^{+}\right], 41(32)\left[\mathrm{C}_{3} \mathrm{H}_{5}^{+}\right]$.

$\mathrm{C}_{12} \mathrm{H}_{27} \mathrm{SiOBr}$ (295.34)

Ber.: C $48.96 \quad$ H 9.25

Gef.: C 48.68 H 9.02 


\section{$5 \quad$ Synthese der $\mathrm{C}_{6}$-Bausteine $(R)-6$ und $(S)-12$}

$5.1 \quad(4 R)-(+)-4-M e t h y l-t e t r a h y d r o-p y r a n-2-o n[(R)-12]^{[88]}$<smiles>CCOC(=O)CC(C)CC(=O)O</smiles>

$(R)-11$
1. $\mathrm{BH}_{3} \bullet \mathrm{SMe}_{2}, \mathrm{THF}, 0{ }^{\circ} \mathrm{C} \rightarrow \mathrm{RT}$, 2. $2 \mathrm{M} \mathrm{KOH}, \mathrm{H}_{2} \mathrm{O} / \mathrm{MeOH}, \mathrm{RT}$

$92 \%$

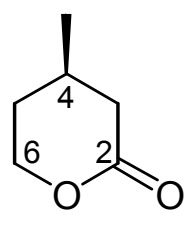

$(R)-12$

$5.23 \mathrm{~g}$ Halbester $(R)-\mathbf{1 1}$ (30.0 mmol, 1.0 Äquiv.) wurden in $40 \mathrm{ml}$ trockenem Tetrahydrofuran (K) gelöst und auf $0{ }^{\circ} \mathrm{C}$ abgekühlt. Nun wurden vorsichtig $21 \mathrm{ml}$ einer Lösung von $2 M$ Boran Dimethylsulfid-Komplex in Tetrahydrofuran (42.0 mmol, 1.4 Äquiv.) zugetropft. Anschließend wurde $30 \mathrm{~min}$ bei $0{ }^{\circ} \mathrm{C}$ und danach $36 \mathrm{~h}$ bei Raumtemperatur gerührt. Nun wurden unter Eiskühlung $15 \mathrm{ml}$ Wasser zugegeben und ca. 20 min gerührt, bis die Wasserstoffentwicklung abgeklungen war. Die Reaktionsmischung wurde am Rotationsverdampfer i. Vak. von Flüchtigem befreit und der verbliebene weiße Rückstand mit $20 \mathrm{ml}$ einer $2 M$ wässrigen Kaliumhydroxidlösung versetzt. Durch Zufügen von 5 - $10 \mathrm{ml}$ Methanol homogenisierte man die Reaktionsmischung und rührte sie $12 \mathrm{~h}$ bei Raumtemperatur. Zum Abschluß säuerte man den Ansatz mit $10 \mathrm{ml}$ einer $6 \mathrm{M}$ Salzsäurelösung an, sättigte die wässrige Phase mit Natriumchlorid und extrahierte viermal mit je $70 \mathrm{ml}$ Diethylether. Die organischen Phasen wurden vereinigt, über Natriumsulfat getrocknet, filtriert und das Lösungsmittel am Rotationsverdampfer i. Vak. entfernt. Das verbliebene Rohprodukt wurde bei $70{ }^{\circ} \mathrm{C}$ im Kugelrohrofen $\left(10^{-2}\right.$ Torr) destilliert. Es wurden 3.15 g (92\% Ausbeute) des Lactons $(R)-12$ als klares Öl isoliert. Der spezifische Drehwinkel von $[\alpha]_{D}^{20}=27.0^{\circ}\left(c=1.0\right.$ in $\left.\mathrm{CHCl}_{3}\right)$ entspricht, verglichen mit den Werten von $\operatorname{Rossi}^{[88]}$, einem Enantiomerenüberschuß von ee $=$ $98 \%\left\{\right.$ Lit. $[\alpha]_{D}^{27}=27.61^{\circ}\left(c=5.716\right.$ in $\left.\left.\mathrm{CHCl}_{3}\right)\right\}$.

$[\alpha]_{D}^{\mathbf{2 0}}=27.0^{\circ}\left(c=1.0\right.$ in $\left.\mathrm{CHCl}_{3}\right),\left\{\right.$ Lit. $^{[88]}[\alpha]_{D}^{27}=27.61^{\circ}\left(c=5.716\right.$ in $\left.\left.\mathrm{CHCl}_{3}\right)\right\}$.

${ }^{1} \mathbf{H}$ NMR $\left(300 \mathrm{MHz}, \mathrm{CDCl}_{3}\right): \delta=1.04\left(\mathrm{~d},{ }^{3} J_{\mathrm{CH}_{3}, \mathrm{CH}}=6.8 \mathrm{~Hz}, 3 \mathrm{H}, \mathrm{CH}_{3}\right), 1.50\left(\mathrm{~m}_{\mathrm{C}}, 1 \mathrm{H}, \mathrm{CH}\right)$, $1.81-2.22\left(\mathrm{~m}, 3 \mathrm{H}, 3-\mathrm{H}_{\mathrm{A}}, 5-\mathrm{H}_{2}\right), 2.65\left(\mathrm{ddd},{ }^{2} J_{3 \mathrm{H}_{\mathrm{B}}, 3 \mathrm{H}_{\mathrm{A}}}=21.0 \mathrm{~Hz},{ }^{3} J_{3 \mathrm{H}_{\mathrm{B}}, 4 \mathrm{H}}=10.5 \mathrm{~Hz},{ }^{4} J_{3 \mathrm{H}_{\mathrm{B}}, 5 \mathrm{H}_{\mathrm{A}}}\right.$ bzw. $\left.{ }^{4} J_{3 \mathrm{H}_{\mathrm{B}}, 5 \mathrm{H}_{\mathrm{B}}}=2.0 \mathrm{~Hz}, 1 \mathrm{H}, 3-\mathrm{H}_{\mathrm{B}}\right), 4.23\left(\mathrm{dt},{ }^{2} J_{6 \mathrm{H}_{\mathrm{A}}, 6 \mathrm{H}_{\mathrm{B}}}={ }^{3} J_{6 \mathrm{H}_{\mathrm{A}}, 5 \mathrm{H}_{\mathrm{A}}}=11.0 \mathrm{~Hz},{ }^{3} J_{6 \mathrm{H}_{\mathrm{A}}, 5 \mathrm{H}_{\mathrm{B}}}=3.5\right.$ $\left.\mathrm{Hz}, 1 \mathrm{H}, 6-\mathrm{H}_{\mathrm{A}}\right), 4.40\left(\mathrm{ddd},{ }^{2} J_{6 \mathrm{H}_{\mathrm{B}}, 6 \mathrm{H}_{\mathrm{A}}}=11.0 \mathrm{~Hz},{ }^{3} J_{6 \mathrm{H}_{\mathrm{B}}, 5 \mathrm{H}_{\mathrm{A}}}=5.0 \mathrm{~Hz},{ }^{3} J_{6 \mathrm{H}_{\mathrm{B}}, 5 \mathrm{H}_{\mathrm{B}}}=3.5 \mathrm{~Hz}, 1 \mathrm{H}, 6-\right.$ $\mathrm{H}_{\mathrm{B}}$. 
${ }^{13} \mathrm{C}$ NMR $\left(50 \mathrm{MHz}, \mathrm{CDCl}_{3}\right): \delta={ }^{+}+21.68\left(4-\mathrm{CH}_{3}\right)$, '+' $26.78(\mathrm{C}-4)$, '-' $30.84(\mathrm{C}-5)$, '-' 38.45 (C-3), '-' $68.84(\mathrm{C}-6)$, '-' $171.52(\mathrm{C}=\mathrm{O})$.

MS (70 eV): $m / z(\%)=114(35)\left[\mathrm{M}^{+}\right], 84(4)\left[\mathrm{M}^{+}-\mathrm{CH}_{2} \mathrm{O}\right], 70(30)\left[\mathrm{M}^{+}-\mathrm{CO}_{2}\right], 69$ (13)

$\left[\mathrm{C}_{4} \mathrm{H}_{5} \mathrm{O}^{+}\right], 55(100)\left[\mathrm{C}_{3} \mathrm{H}_{3} \mathrm{O}^{+}\right], 43(10)\left[\mathrm{C}_{3} \mathrm{H}_{7}^{+}\right], 42(73)\left[\mathrm{C}_{2} \mathrm{H}_{2} \mathrm{O}^{+}\right]$.

$\mathrm{C}_{6} \mathrm{H}_{10} \mathrm{O}_{4}$ (114.14)

\section{$5.2 \quad(3 R)-(+)-5-H y d r o x y-3-m e t h y l-1-p i p e r i d i n-1-y l-p e n t a n-1-o n ~[(R)-56]$}

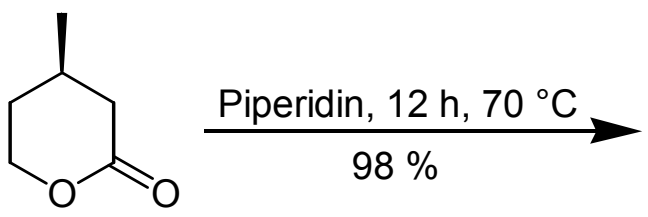

$(R)-12$<smiles>CC(CCO)CC(=O)N1CCCCC1</smiles>

$(R)-56$

2.8 g Lacton (25.0 mmol, 1.0 Äquiv.) (R)-12 wurden mit $17 \mathrm{~g}$ (200 mmol, 8.0 Äquiv.) frisch destilliertem Piperidin $\left(\mathrm{CaH}_{2}\right)$ versetzt und in einem Druckkolben unter Rühren für $12 \mathrm{~h}$ auf $70^{\circ} \mathrm{C}$ erhitzt. Abschließend ließ man abkühlen und entfernte das überschüssige Piperidin i. Vak. am Rotationsverdampfer. Das erhaltene Rohprodukt konnte ohne weitere Aufreinigung für die nächste Syntheseoperation eingesetzt werden. Analysenreine Substanz erhielt man durch Chromatographie des rohen Piperidids an Kieselgel mit Aceton als Laufmittel. Geringe Mengen dabei sich lösenden Kieselgels ließen sich durch abschließende Filtration über Celite einfach entfernen. Nach der Aufreinigung des Rohprodukts erhielt man 4.79 g (98 \% Ausbeute) Piperidid ( $R$ )-56 als farbloses Öl.

$\mathbf{R}_{\mathbf{f}}=0.2(\mathrm{EE} / \mathrm{PE}=20: 1)$.

$[\alpha]_{D}^{\mathbf{2 0}}=9.4^{\circ}\left(c=0.8\right.$ in $\left.\mathrm{CHCl}_{3}\right),\left\{\right.$ Lit. $^{[115]}[\alpha]_{D}^{\mathbf{2 0}}=7.9^{\circ}\left(c=3\right.$ in $\left.\left.\mathrm{CHCl}_{3}\right)\right\}$.

IR (Film): $\widetilde{v}=3406 \mathrm{~cm}^{-1}(\mathrm{O}-\mathrm{H}), 2933,2857$ (C-H), 1620 (Amid), 1444, 1369, $\left(\mathrm{CH}_{2}, \mathrm{CH}_{3}\right)$, 1059, 1020 (C-O).

$\mathbf{U V}\left(\mathrm{CH}_{3} \mathrm{CN}\right): \lambda_{\max }(\lg \varepsilon)=205 \mathrm{~nm}(3.92)$.

${ }^{1} \mathbf{H}$ NMR $\left(200 \mathrm{MHz}, \mathrm{CDCl}_{3}\right): \delta=0.95\left(\mathrm{~d},{ }^{3} J_{\mathrm{CH}_{3}, \mathrm{CH}}=6.8 \mathrm{~Hz}, 3 \mathrm{H}, \mathrm{CH}_{3}\right), 1.32-1.70(\mathrm{~m}, 8 \mathrm{H}, 2$ '$\left.\mathrm{H}_{2}, 3^{\prime}-\mathrm{H}_{2}, 4^{\prime}-\mathrm{H}_{2}, 3-\mathrm{H}, 4-\mathrm{H}_{\mathrm{B}}\right), 2.07$ - 2.39 (m, 3H, 2- $\mathrm{H}_{2}, 4-\mathrm{H}_{\mathrm{A}}$ ), 3.16 (s br, 1H, OH); 3.37 (t br, ${ }^{3} J_{1}{ }^{\prime} \mathrm{H}_{2},{ }^{\prime} \mathrm{H}_{2}=5.1 \mathrm{~Hz}, 2 \mathrm{H}, 1$ ' $-\mathrm{H}_{2}$ bzw. 5'- $\left.-\mathrm{H}_{2}\right), 3.51$ (t br, ${ }^{3} J_{5}{ }^{\prime} \mathrm{H}_{2}, 4^{\prime} \mathrm{H}_{2}=5.0 \mathrm{~Hz}, 2 \mathrm{H}, 5^{\prime}-\mathrm{H}_{2}$ bzw. 1'$\left.\mathrm{H}_{2}\right), 3.57\left(\mathrm{t},{ }^{3} \mathrm{~J}_{5 \mathrm{H}_{2}, 4 \mathrm{H}_{2}}=6.0 \mathrm{~Hz}, 2 \mathrm{H}, 5-\mathrm{H}_{2}\right)$. 


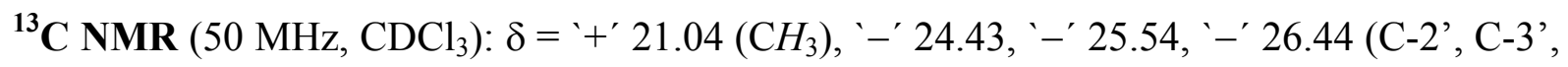
C-4'), '+' $26.48(\mathrm{CH})$, '-' $^{\prime} 40.06$ (C-2 und C-4), '-' 42.82 (C-1'), '-' 46,75 (C-5'), '-' 60.13 (C-5), ’’' $171.16(\mathrm{C}-1)$.

MS $(70 \mathrm{eV}): m / z(\%)=199(15)\left[\mathrm{M}^{+}\right], 181(17)\left[\mathrm{M}^{+}-\mathrm{H}_{2} \mathrm{O}\right], 168(10)\left[\mathrm{M}^{+}-\mathrm{CH}_{3} \mathrm{O}\right], 154(42)$ $\left[\mathrm{M}^{+}-\mathrm{C}_{2} \mathrm{H}_{5} \mathrm{O}\right], 127(100)\left[\mathrm{C}_{7} \mathrm{H}_{13} \mathrm{NO}^{+}\right], 112(68)\left[\mathrm{C}_{6} \mathrm{H}_{10} \mathrm{NO}^{+}\right], 99$ (6) [127 - CO], 84 (56) $\left[\mathrm{C}_{5} \mathrm{H}_{10} \mathrm{~N}^{+}\right], 71(6)\left[\mathrm{C}_{4} \mathrm{H}_{7} \mathrm{O}^{+}\right], 69(30)\left[\mathrm{C}_{5} \mathrm{H}_{9}{ }^{+}\right], 56(10)\left[\mathrm{C}_{3} \mathrm{H}_{6} \mathrm{~N}^{+}\right], 41(23)\left[\mathrm{C}_{3} \mathrm{H}_{5}^{+}\right]$.

$\mathbf{C}_{11} \mathrm{H}_{21} \mathrm{NO}_{2}$ (199.29) $\quad$ Ber.: C $66.29 \quad$ H 10.62

Gef.: C $66.47 \quad$ H 10.87

5.3 (3R)-(-)-5-tert.-Butyldimethylsilyloxy-3-methyl-1-piperidin-yl-pentan-1-on [(R)-57)]

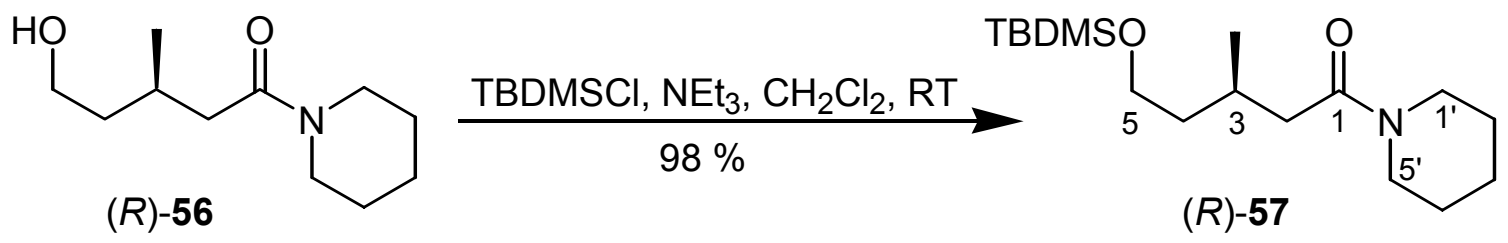

Es wurden $4.50 \mathrm{~g}$ (22.5 mmol, 1.0 Äquiv.) des Piperidids $(R)-56$ in $40 \mathrm{ml}$ Dichlormethan gelöst und auf $0{ }^{\circ} \mathrm{C}$ abgekühlt. Bei dieser Temperatur fügte man zunächst $3.73 \mathrm{~g}(24.8 \mathrm{mmol}$, 1.1 Äquiv.) tert.-Butyldimethylchlorsilan und dann tropfenweise $4.05 \mathrm{ml}$ (2.96 g, $29.3 \mathrm{mmol}$, 1.5 Äquiv.) frisch destilliertes Triethylamin zu. Die Reaktionsmischung wurde auf Raumtemperatur erwärmt und der Fortgang der Reaktion mittels dünnschichtchromatographischer Analyse verfolgt $(\mathrm{EE} / \mathrm{PE}=5: 1)$. Nach annähernd $4 \mathrm{~h}$ war das Substrat vollständig verbraucht. Zur Aufarbeitung verdünnte man den Ansatz mit $50 \mathrm{ml}$ Dichlormethan, wusch einmal mit $20 \mathrm{ml}$ gesättigter Natriumhydrogencarbonatlösung und trocknete die organische Phase über Natriumsulfat. Abfiltrieren des Trockenmittels und Entfernen des Solvens i. Vak. ergaben 7.58 g Rohprodukt, das an $450 \mathrm{~g}$ Kieselgel chromatographiert wurde $\left(\mathrm{Et}_{2} \mathrm{O} / \mathrm{PE}=1: 5\right.$ $\rightarrow \mathrm{Et}_{2} \mathrm{O}$ ). Man isolierte $6.95 \mathrm{~g}$ (98\% Ausbeute) der gewünschten Verbindung $(R)-57$ als klares Öl.

$\mathbf{R}_{\mathbf{f}}=0.60(\mathrm{EE} / \mathrm{PE}=5: 1)$.

$[\alpha]_{D}^{\mathbf{2 0}}=-1.5^{\circ}\left(c=1.0\right.$ in $\left.\mathrm{CHCl}_{3}\right)$. 
IR (Film): $\widetilde{v}=2931 \mathrm{~cm}^{-1}, 2856\left(\mathrm{CH}_{2}, \mathrm{CH}_{3}\right), 1646$ (Amid), 1462, $1433\left(\mathrm{CH}_{2}, \mathrm{CH}_{3}\right), 1254$, 1095 (C-O).

UV $\left(\mathrm{CH}_{3} \mathrm{CN}\right): \lambda_{\max }(\lg \varepsilon)=205 \mathrm{~nm}(3.91)$.

${ }^{1} \mathbf{H}$ NMR $\left.\left(200 \mathrm{MHz}, \mathrm{CDCl}_{3}\right): \delta=0.00\left[\mathrm{~s}, 6 \mathrm{H}, \mathrm{Si}\left(\mathrm{CH}_{3}\right)_{2}\right], 0.85\left[\mathrm{~s}, 9 \mathrm{H}, \mathrm{SiC}\left(\mathrm{CH}_{3}\right)_{3}\right)\right], 0.93(\mathrm{~d}$, $\left.{ }^{3} J_{\mathrm{CH}_{3}, \mathrm{CH}}=6.5 \mathrm{~Hz}, 3 \mathrm{H}, \mathrm{CH}_{3}\right), 1.29-1.70\left(\mathrm{~m}, 8 \mathrm{H}, 2^{\prime}-\mathrm{H}_{2}, 3^{\prime}-\mathrm{H}_{2}, 4^{\prime}-\mathrm{H}_{2}, 3-\mathrm{H}, 4-\mathrm{H}_{\mathrm{B}}\right), 2.08(\mathrm{dt}$, $\left.{ }^{2} J_{4 \mathrm{H}_{\mathrm{A}}, 4 \mathrm{H}_{\mathrm{B}}}=13.0 \mathrm{~Hz},{ }^{3} J_{4 \mathrm{H}_{\mathrm{A}}, 5 \mathrm{H}_{\mathrm{A}}}={ }^{3} J_{4 \mathrm{H}_{\mathrm{A}}, 5 \mathrm{H}_{\mathrm{B}}}=6.0 \mathrm{~Hz}, 1 \mathrm{H}, 4-\mathrm{H}_{\mathrm{A}}\right)$ überlagert von $2.08(\mathrm{dd}$, $\left.{ }^{2} J_{2 \mathrm{H}_{\mathrm{A}}, 2 \mathrm{H}_{\mathrm{B}}}=17.5 \mathrm{~Hz},{ }^{3} J_{2 \mathrm{H}_{\mathrm{A}}, 3 \mathrm{H}}=9.0 \mathrm{~Hz}, 1 \mathrm{H}, 2-\mathrm{H}_{\mathrm{A}}\right), 2.37\left(\mathrm{dd},{ }^{2} J_{2 \mathrm{H}_{\mathrm{B}}, 2 \mathrm{H}_{\mathrm{A}}}=17.5 \mathrm{~Hz},{ }^{3} J_{2 \mathrm{H}_{\mathrm{B}}, 3 \mathrm{H}}=9.0\right.$ $\left.\mathrm{Hz}, 1 \mathrm{H}, 2-\mathrm{H}_{\mathrm{B}}\right), 3.36\left(\mathrm{t} \mathrm{br},{ }^{3} J^{\prime} \mathrm{H}_{2}, 2^{\prime} \mathrm{H}_{2}=5.0 \mathrm{~Hz}, 2 \mathrm{H}, 1^{\prime}-\mathrm{H}_{2}\right.$ bzw. $\left.5^{\prime}-\mathrm{H}_{2}\right), 3.51\left(\mathrm{t} \mathrm{br},{ }^{3} J_{5}{ }^{\prime} \mathrm{H}_{2}, 4^{\prime} \mathrm{H}_{2}=\right.$ $5.0 \mathrm{~Hz}, 2 \mathrm{H}, 5$ ' $-\mathrm{H}_{2}$ bzw. 1'- $\left.-\mathrm{H}_{2}\right), 3.63\left(\mathrm{t},{ }^{3} J_{5 \mathrm{H}_{\mathrm{B}}, 4 \mathrm{H}_{\mathrm{A}}}={ }^{3} J_{5 \mathrm{H}_{\mathrm{B}}, 4 \mathrm{H}_{\mathrm{B}}}=6.0 \mathrm{~Hz}, 1 \mathrm{H}, 5-\mathrm{H}_{\mathrm{B}}\right), 3.64(\mathrm{t}$, $\left.{ }^{3} J_{5 \mathrm{H}_{\mathrm{A}}, 4 \mathrm{H}_{\mathrm{A}}}={ }^{3} J_{5 \mathrm{H}_{\mathrm{A}}, 4 \mathrm{H}_{\mathrm{B}}}=6.0 \mathrm{~Hz}, 1 \mathrm{H}, 5-\mathrm{H}_{\mathrm{A}}\right)$.

${ }^{13} \mathrm{C}$ NMR $\left.\left(50 \mathrm{MHz}, \mathrm{CDCl}_{3}\right): \delta={ }^{+}+5.35\left[\mathrm{Si}\left(\mathrm{CH}_{3}\right)_{2}\right)\right],{ }^{-}-18.25\left[\mathrm{SiC}\left(\mathrm{CH}_{3}\right)_{3}\right],{ }^{\prime}+{ }^{\prime} 19.93$ $\left(\mathrm{CH}_{3}\right)$, '-' 24.85, '-' 25.65, `-' $26.62\left(\mathrm{C}-2\right.$ ', C-3', C-4'), '+' $25.91\left[\mathrm{SiC}\left(\mathrm{CH}_{3}\right)_{3}\right]$, '+' 27.39 (CH), '-' 39.83 (C-2 oder C-4), '-' 40.88 (C-4 oder C-2), '-' 42.57 (C-1'), '-' 46.90 (C-5'), '-' $61.12(\mathrm{C}-5)$, '-' $170.68(\mathrm{C}-1)$.

MS $(70 \mathrm{eV}): m / z(\%)=313(26)\left[\mathrm{M}^{+}\right], 298(3)\left[\mathrm{M}^{+}-\mathrm{CH}_{3}\right], 256(100)\left[\mathrm{M}^{+}\right.$-tert-Butyl], $182(4)$ [M $\left.\mathrm{M}^{+}-\mathrm{TBDMSO}\right], 154$ (3) $\left[\mathrm{C}_{9} \mathrm{H}_{16} \mathrm{NO}^{+}\right], 142$ (4) [256- $\left.\mathrm{C}_{5} \mathrm{H}_{10} \mathrm{SiO}\right], 127$ (3) $\left[\mathrm{C}_{7} \mathrm{H}_{13} \mathrm{NO}^{+}\right], 112$ (2) $\left[\mathrm{C}_{6} \mathrm{H}_{10} \mathrm{NO}^{+}\right], 84(4)\left[\mathrm{C}_{5} \mathrm{H}_{10} \mathrm{~N}^{+}\right], 73(7)\left[\mathrm{C}_{2} \mathrm{H}_{5} \mathrm{SiO}^{+}\right], 41(6)\left[\mathrm{C}_{3} \mathrm{H}_{5}^{+}\right]$.

$\begin{array}{lll}\mathbf{C}_{17} \mathrm{H}_{35} \mathbf{N O}_{2} \mathrm{Si} \text { (313.56) } & \text { Ber.: C } 65.12 & \text { H } 11.25 \\ & \text { Gef.: C } 65.27 & \text { H } 11.18\end{array}$

\section{4 (3S)-(+)-5-tert.-Butyldimethylsilyoxy-3-methyl-pentan-1-ol [(S)-52]}

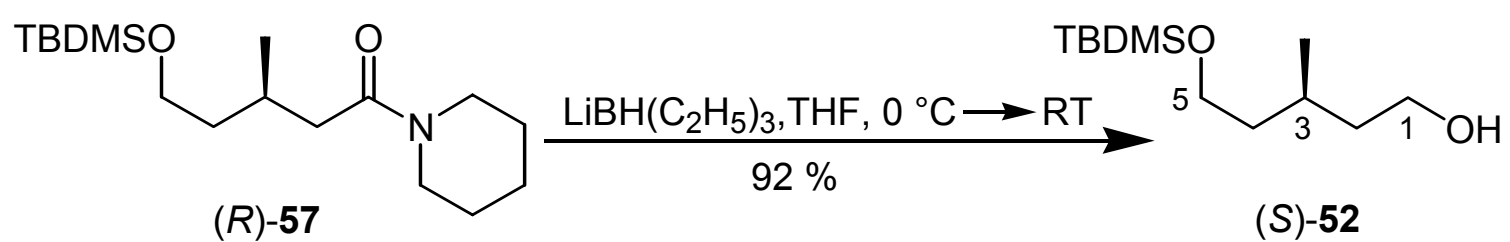

In einem Dreihalskolben wurden $46.5 \mathrm{ml}$ (46.5 mmol, 2.5 Äquiv.) einer $1 M$ Lösung von $\mathrm{LiBH}\left(\mathrm{C}_{2} \mathrm{H}_{5}\right)_{3}$ (Superhydride $\left.{ }^{\circledR}\right)$ auf $0{ }^{\circ} \mathrm{C}$ abgekühlt. Dann wurden $5.86 \mathrm{~g}$ (18.7 mmol, 1.0 Äquiv.) des Substrats (R)-57 in $30 \mathrm{ml}$ Tetrahydrofuran (K) unter kräftigem Rühren, mit Hilfe eines Tropftrichters eingetropft. Nach $1 \mathrm{~h}$ bei $0^{\circ} \mathrm{C}$ ließ man die Reaktionsmischung auf 
Raumtemperatur auftauen und rührte etwa $1 \mathrm{~h}$, bis laut dünnschichtchromatographischer Analyse $(\mathrm{EE} / \mathrm{PE}=1: 3)$ das Amid vollständig reduziert war. Dann wurde bei $0{ }^{\circ} \mathrm{C}$ die Reaktion durch Zugabe von $30 \mathrm{ml}$ gesättigter Ammoniumchloridlösung gequencht und die Reaktionsmischung anschließend mit $100 \mathrm{ml}$ Dichlormethan in einen Scheidetrichter übergeführt. Nach dem Abtrennen der organischen Phase sättigte man die wässrige Phase mit Natriumsulfat und extrahiert diese noch dreimal mit je $100 \mathrm{ml}$ Dichlormethan. Die vereinigten organischen Phasen wurden über Natriumsulfat getrocknet, filtriert und am Rotationsverdampfer i. Vak. eingeengt. Die Reinigung des Rohproduktes erfolgte durch Chromatographie an $500 \mathrm{~g}$ Kieselgel. Als Laufmittel diente ein Gemisch von $\mathrm{Et}_{2} \mathrm{O} / \mathrm{PE}=1: 3$. Es wurden $4.00 \mathrm{~g}$ (92\% Ausbeute) der gewünschten Verbindung (S)-52 erhalten.

$\mathbf{R}_{\mathbf{f}}=0.43(\mathrm{EE} / \mathrm{PE}=1: 3)$.

$[\alpha]_{D}^{20}=2.3^{\circ}\left(c=1.0\right.$ in $\left.\mathrm{CHCl}_{3}\right)$.

IR (Film): $\widetilde{v}=3353 \mathrm{~cm}^{-1}(\mathrm{OH}), 2955,2929,2885,2858\left(\mathrm{CH}_{2}, \mathrm{CH}_{3}\right), 1471,1462\left(\mathrm{CH}_{2}, \mathrm{CH}_{3}\right)$, 1255 (C-OSi), 1096 (C-O), 836 (Si-OC), 775 (C-SiCH 3$).$

UV $\left(\mathrm{CH}_{3} \mathrm{CN}\right)$ : Die Verbindung $(S)-52$ zeigt im Wellenlängenbereich von 195 bis $400 \mathrm{~nm}$ keine UV-Absorption.

${ }^{1} \mathbf{H}$ NMR $\left(300 \mathrm{MHz}, \mathrm{C}_{6} \mathrm{D}_{6}\right): \delta=0.17$ [s, 6H, $\left.\mathrm{Si}\left(\mathrm{CH}_{3}\right)_{2}\right], 0.93$ (s br, $\left.1 \mathrm{H}, \mathrm{OH}\right)$, teilweise überlagert von $0.94\left(\mathrm{~d},{ }^{3} J_{\mathrm{CH}_{3}, \mathrm{CH}}=6.5 \mathrm{~Hz}, 3 \mathrm{H}, \mathrm{CH}_{3}\right), 1.09\left[\mathrm{~s}, 9 \mathrm{H}, \mathrm{SiC}\left(\mathrm{CH}_{3}\right)\right], 1.26-1.47(\mathrm{~m}$, $\left.2 \mathrm{H}, 3-\mathrm{H}, 2-\mathrm{H}_{\mathrm{B}}\right), 1.55\left(\mathrm{dq},{ }^{2} J_{4 \mathrm{H}_{\mathrm{B}}, 4 \mathrm{H}_{\mathrm{A}}}=14.0 \mathrm{~Hz},{ }^{3} J_{4 \mathrm{H}_{\mathrm{B}}, 3 \mathrm{H}}={ }^{3} J_{4 \mathrm{H}_{\mathrm{B}}, 5 \mathrm{H}_{2}}=7.0 \mathrm{~Hz}, 1 \mathrm{H}, 4-\mathrm{H}_{\mathrm{B}}\right), 1.65$ $\left(\mathrm{dtd},{ }^{2} J_{4 \mathrm{H}_{\mathrm{A}}, 4 \mathrm{H}_{\mathrm{B}}}=14.0 \mathrm{~Hz},{ }^{3} J_{4 \mathrm{H}_{\mathrm{A}}, 3 \mathrm{H}}={ }^{3} J_{4 \mathrm{H}_{\mathrm{A}}, 5 \mathrm{H}_{\mathrm{A}}}=7.0 \mathrm{~Hz},{ }^{3} J_{4 \mathrm{H}_{\mathrm{A}}, 5 \mathrm{H}_{\mathrm{B}}}=6.0 \mathrm{~Hz}\right.$ bzw. ${ }^{2} J_{4 \mathrm{H}_{\mathrm{A}}, 4 \mathrm{H}_{\mathrm{B}}}=$ $\left.14.0 \mathrm{~Hz},{ }^{3} J_{4 \mathrm{H}_{\mathrm{A}}, 5 \mathrm{H}_{2}}=7.0 \mathrm{~Hz},{ }^{3} J_{4 \mathrm{H}_{\mathrm{A}}, 3 \mathrm{H}}=6.0 \mathrm{~Hz}, 1 \mathrm{H}, 4-\mathrm{H}_{\mathrm{A}}\right), 1.84\left(\mathrm{ddt},{ }^{2} J_{2 \mathrm{H}_{\mathrm{A}}, 2 \mathrm{H}_{\mathrm{B}}}=20.5 \mathrm{~Hz}\right.$, ${ }^{3} J_{2 \mathrm{H}_{\mathrm{A}}, 1 \mathrm{H}_{\mathrm{A}}}=14.0 \mathrm{~Hz},{ }^{3} J_{2 \mathrm{H}_{\mathrm{A}}, 1 \mathrm{H}_{\mathrm{B}}}=6.5 \mathrm{~Hz}, 1 \mathrm{H}, 2-\mathrm{H}_{\mathrm{A}}$ ), $3.45-3.60$ (m schwach aufgelöst, 2H, 1$\mathrm{H}_{2}$ bzw. 5- $\left.\mathrm{H}_{2}\right), 3.68\left(\mathrm{~m}_{\mathrm{C}}, 2 \mathrm{H}, 5-\mathrm{H}_{2}\right.$ bzw. 1- $\left.\mathrm{H}_{2}\right)$.

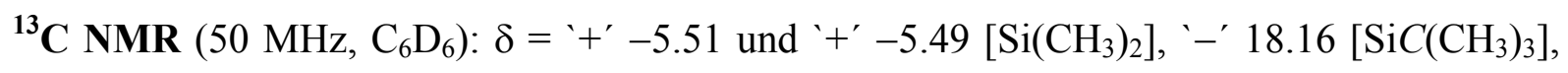

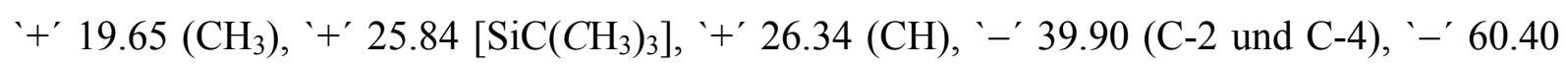
(C-1), '-' $61.09(\mathrm{C}-5)$.

MS $\left(200 \mathrm{eV}, \mathrm{DCI} / \mathrm{NH}_{3}\right): m / z(\%)=482(5)\left[2 \mathrm{M}+\mathrm{NH}_{4}^{+}\right], 250(100)\left[\mathrm{M}+\mathrm{NH}_{4}^{+}\right], 233(40)$ $\left[\mathrm{M}+\mathrm{H}^{+}\right]$.

\section{$\mathrm{C}_{12} \mathrm{H}_{28} \mathrm{SiO}_{2}(232.44)$}




\section{5 (3R)-(-)-tert.-Butyl-(5-iodo-3-methyl-pentyloxy)-dimethyl-silan $[(R)-6]$}

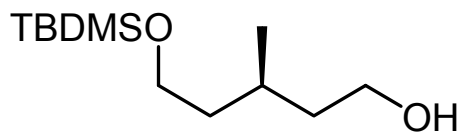

(S)-52

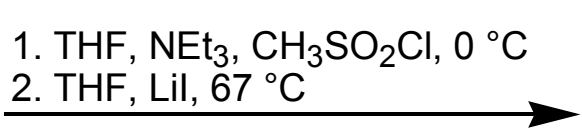

$93 \%$

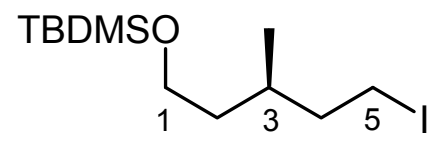

$(R)-6$

Es wurden $232 \mathrm{mg}$ (1.0 mmol, 1.0 Äquiv.) Alkohol $(S)$-52 in $5 \mathrm{ml}$ trockenem Tetrahydrofuran (K) gelöst und auf $0{ }^{\circ} \mathrm{C}$ abgekühlt. Dann versetzte man die kalte Lösung mit $126 \mathrm{mg}$ (1.1 mmol, 1.1 Äquiv.) Methansulfonsäurechlorid und $194 \mu \mathrm{l}$ (142 mg, 1.3 mmol, 1.3 Äquiv.) frisch destilliertem Triethylamin. Nach 15 min bei $0{ }^{\circ} \mathrm{C}$ war die Reaktion beendet (DC Kontrolle: $\mathrm{Et}_{2} \mathrm{O} / \mathrm{PE}=1: 2$ ). Man nahm die Reaktionsmischung in $10 \mathrm{ml}$ gesättigter Natriumhydrogencarbonatlösung auf und extrahierte viermal mit je $10 \mathrm{ml}$ Diethylether. Die getrockneten organischen Phasen wurden filtriert und das Lösungsmittel i. Vak. am Rotationsverdampfer entfernt. Das rohe Mesylat wurde ohne Aufreinigung in $20 \mathrm{ml}$ Tetrahydrofuran gelöst, auf $0{ }^{\circ} \mathrm{C}$ abgekühlt, mit $161 \mathrm{mg}$ (1.2 mmol, 1.2 Äquiv.) wasserfreiem Lithiumiodid versetzt und unter Sieden am Rückfluß erhitzt. Nach ca. 2 h hatte das Mesylat vollständig abreagiert (DC Kontrolle: $\mathrm{Et}_{2} \mathrm{O} / \mathrm{PE}=1: 2$ ). Die erkaltete Reaktionslösung wurde in $20 \mathrm{ml}$ Wasser aufgenommen und viermal mit je $20 \mathrm{ml}$ Diethylether extrahiert und die organischen Phasen über Natriumsulfat getrocknet, filtriert und i. Vak. am Rotationsverdampfer vom Lösungsmittel befreit. Zur Reinigung wurde das rohe Iodid an $20 \mathrm{~g}$ Kieselgel chromatographiert $\left(\mathrm{Et}_{2} \mathrm{O} / \mathrm{PE}=1: 100\right)$. Es wurden $318 \mathrm{mg}$ der Titelverbindung $(R)$ 6 als Ö1 isoliert. Die Ausbeute betrug $93 \%$.

$[\alpha]_{D}^{\mathbf{2 0}}=-11.0^{\circ}\left(c=1.0\right.$ in $\left.\mathrm{CHCl}_{3}\right)$.

${ }^{1} \mathbf{H}$ NMR $\left(200 \mathrm{MHz}, \mathrm{C}_{6} \mathrm{D}_{6}\right): \delta=0.15,\left[\mathrm{~s}, 6 \mathrm{H}, \mathrm{Si}\left(\mathrm{CH}_{3}\right)_{2}\right], 0.73\left(\mathrm{~d},{ }^{3} J_{3 \mathrm{CH}_{3}, 3 \mathrm{H}}=6.5 \mathrm{~Hz}, 3 \mathrm{H}, 3-\right.$ $\left.\mathrm{CH}_{3}\right), 1.08$ [s, 9H, $\left.\mathrm{SiC}\left(\mathrm{CH}_{3}\right)_{3}\right], 1.10-1.35(\mathrm{~m}, 1 \mathrm{H}, \mathrm{CH}), 1.35$ - $1.55\left(\mathrm{~m}, 2 \mathrm{H}, 2-\mathrm{H}_{2}\right.$ oder 4- $\left.\mathrm{H}_{2}\right)$, $1.55-1.85\left(\mathrm{~m}, 2 \mathrm{H}, 4-\mathrm{H}_{2}\right.$ oder $\left.2-\mathrm{H}_{2}\right), 2.83 \mathrm{ddd},{ }^{2} J_{5 \mathrm{H}_{\mathrm{B}}, 5 \mathrm{H}_{\mathrm{A}}}=15.0 \mathrm{~Hz},{ }^{3} J_{5 \mathrm{H}_{\mathrm{B}}, 5 \mathrm{H}_{\mathrm{A}}}=9.5 \mathrm{~Hz}$, $\left.{ }^{3} J_{5 \mathrm{H}_{\mathrm{B}}, 5 \mathrm{H}_{\mathrm{A}}}=7.0 \mathrm{~Hz}, 1 \mathrm{H}, 5-\mathrm{H}_{\mathrm{B}}\right)$ überlagert von $2.91\left(\mathrm{ddd},{ }^{2} J_{5 \mathrm{H}_{\mathrm{B}}, 5 \mathrm{H}_{\mathrm{A}}}=15.0 \mathrm{~Hz},{ }^{3} J_{5 \mathrm{H}_{\mathrm{B}}, 5 \mathrm{H}_{\mathrm{A}}}=9.0\right.$ $\left.\mathrm{Hz},{ }^{3} J_{5 \mathrm{H}_{\mathrm{B}}, 5 \mathrm{H}_{\mathrm{A}}}=5.5 \mathrm{~Hz}, 1 \mathrm{H}, 5-\mathrm{H}_{\mathrm{B}}\right), 3.57\left(\mathrm{t},{ }^{3} \mathrm{~J}_{1 \mathrm{H}_{2}, 2 \mathrm{H}_{2}}=6.5 \mathrm{~Hz}, 2 \mathrm{H}, 1-\mathrm{H}_{2}\right)$.

${ }^{13} \mathrm{C}$ NMR $\left(50 \mathrm{MHz}, \mathrm{C}_{6} \mathrm{D}_{6}\right): \delta={ }^{+}{ }^{\prime}-5.24$ und '+' $-5.20\left(\mathrm{Si}\left(\mathrm{CH}_{3}\right)_{2}\right)$, `' $4.61(\mathrm{C}-5)$, `' 18.42

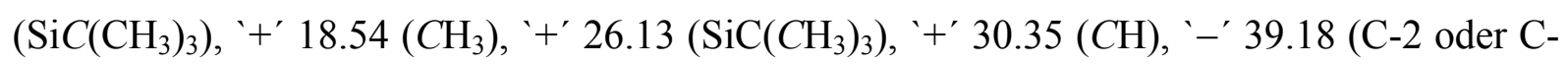
4), '-' 41.07 (C-4 oder C-2), '-' 60.88 (C-1).

\section{$\mathrm{C}_{12} \mathrm{H}_{27} \mathrm{SiOI}(342.09)$}




\subsection{Synthese des Lactons (4S)-(-)-4-Methyl-tetrahydro-pyran-2-on $[(S)-12)]^{[90]}$}<smiles>CCOC(=O)C[C@H](C)CC(=O)O</smiles>

$(R)-11$
1. $\mathrm{LiBH}_{4} \mathrm{MeOH}, \mathrm{DME}, 85^{\circ} \mathrm{C}$ 2. $2 \mathrm{M} \mathrm{NaOH}, \mathrm{RT}$

$84 \%$

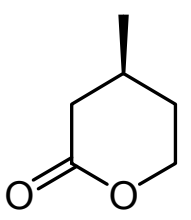

(S)-12

In $2 \mathrm{ml}$ trockenem 1,2-Dimethoxyethan ( $\mathrm{Na}$ ) wurden $34 \mathrm{mg}$ (1.5 mmol, 1.5 Äquiv.) 95 proz. Lithiumborhydrid suspendiert. Dazu spritzte man vorsichtig eine Lösung aus $61 \mu 1$ (48 mg, 1.5 mol, 1.5 Äquiv.) trockenem Methanol (Na) und $174 \mathrm{mg}$ (1.0 mmol, 1.0 Äquiv.) Halbester (R)-11 in $2 \mathrm{ml} \mathrm{1,2-Dimethoxyethan.} \mathrm{Die} \mathrm{Lösung} \mathrm{wurde} 3 \mathrm{~h}$ unter Rückfluß zum Sieden erhitzt, dann auf $0{ }^{\circ} \mathrm{C}$ abgekühlt und mit $0.2 \mathrm{ml}$ einer $1 \mathrm{M}$ Salzsäurelösung gequencht. Die Reaktionsmischung wurde anschließend am Rotationsverdampfer bis zur Trockene eingeengt und der Rückstand in $1.0 \mathrm{ml}$ einer $2 M$ Natriumhydroxidlösung aufgenommen. Nach $8 \mathrm{~h}$ Rühren bei Raumtemperatur säuerte man mit $0.4 \mathrm{ml}$ einer $6 M$ Salzsäurelösung an und extrahierte viermal mit je $10 \mathrm{ml}$ Dichlormethan. Die vereinigten organischen Phasen wurden über Natriumsulfat getrocknet, filtriert und das Solvens i. Vak. am Rotationsverdampfer entfernt. Das Rohprodukt wurde bei $70{ }^{\circ} \mathrm{C}$ im Kugelrohrofen $\left(10^{-2}\right.$ Torr $)$ destilliert. So erhielt man 96 mg (84\% Ausbeute ) des Lactons $(S)$-12 als klares Öl. Der spezifische Drehwinkel von $[\alpha]_{D}^{20}=-24.0^{\circ}\left(c=1.0\right.$ in $\left.\mathrm{CHCl}_{3}\right)$ entspricht, verglichen mit den Werten von Nohira, ${ }^{[91]}$ einem Enantiomerenüberschuß von ee $=89 \%\left\{\right.$ Lit. $[\alpha]_{D}^{20}=-27.0^{\circ}\left(c=1\right.$ in $\left.\left.\mathrm{CHCl}_{3}\right)\right\}$.

$[\alpha]_{D}^{\mathbf{2 0}}=-24.0^{\circ}\left(c=1.0\right.$ in $\left.\mathrm{CHCl}_{3}\right),\left\{\right.$ Lit. $^{[91]}[\alpha]_{D}^{\mathbf{2 0}}=-27.0^{\circ}\left(c=1\right.$ in $\left.\left.\mathrm{CHCl}_{3}\right)\right\}$.

${ }^{1} \mathbf{H}$ NMR $\left(200 \mathrm{MHz}, \mathrm{CDCl}_{3}\right): \delta=1.00\left(\mathrm{~d},{ }^{3} \mathrm{~J}_{\mathrm{CH}_{3}, \mathrm{CH}}=6.5 \mathrm{~Hz}, 3 \mathrm{H}, \mathrm{CH}_{3}\right), 1.32-1.57(\mathrm{~m}, 1 \mathrm{H}$, $\mathrm{CH}), 1.75-2.18\left(\mathrm{~m}, 3 \mathrm{H}, 3-\mathrm{H}_{\mathrm{B}}, 5-\mathrm{H}_{2}\right), 2.61\left(\mathrm{ddd},{ }^{2} J_{3 \mathrm{H}_{\mathrm{A}}, 3 \mathrm{H}_{\mathrm{A}}}=21.0 \mathrm{~Hz},{ }^{3} J_{3 \mathrm{H}_{\mathrm{A}}, 3 \mathrm{H}}=10.5 \mathrm{~Hz}\right.$, ${ }^{4} J_{3 \mathrm{H}_{\mathrm{A}}, 4 \mathrm{H}_{\mathrm{A}}}$ bzw. $\left.{ }^{4} J_{3 \mathrm{H}_{\mathrm{A}}, 4 \mathrm{H}_{\mathrm{B}}}=2.0 \mathrm{~Hz}, 1 \mathrm{H}, 3-\mathrm{H}_{\mathrm{A}}\right), 4.20\left(\mathrm{dt},{ }^{2} J_{6 \mathrm{H}_{\mathrm{B}}, 6 \mathrm{H}_{\mathrm{A}}}={ }^{3} J_{6 \mathrm{H}_{\mathrm{B}}, 5 \mathrm{H}_{\mathrm{A}}}=11.5 \mathrm{~Hz}\right.$,

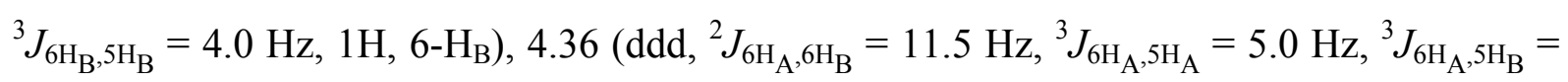
4.0 Hz, $\left.1 \mathrm{H}, 6-\mathrm{H}_{\mathrm{A}}\right)$.

${ }^{13}$ C NMR $\left(50 \mathrm{MHz}, \mathrm{CDCl}_{3}\right): \delta=$ '+' $21.32\left(\mathrm{CH}_{3}\right)$, '+' 26.42 (C-4 ), '-' 30.48 (C-5), '-' 38.09 (C-3), '-' 68.49 (C-6), '-' 171.19 (C=O).

$\mathrm{C}_{6} \mathrm{H}_{10} \mathrm{O}_{2}$ (114.15) 


\section{Synthese des diastereomerenreinen Vinyliodids 7}

\section{1 (5E,9E)-6,10,14-Trimethyl-pentadeca-5,9,13-trien-1-in $(9)^{[99,100]}$}

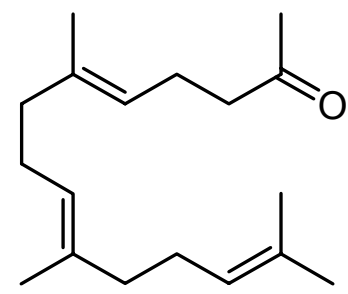

10

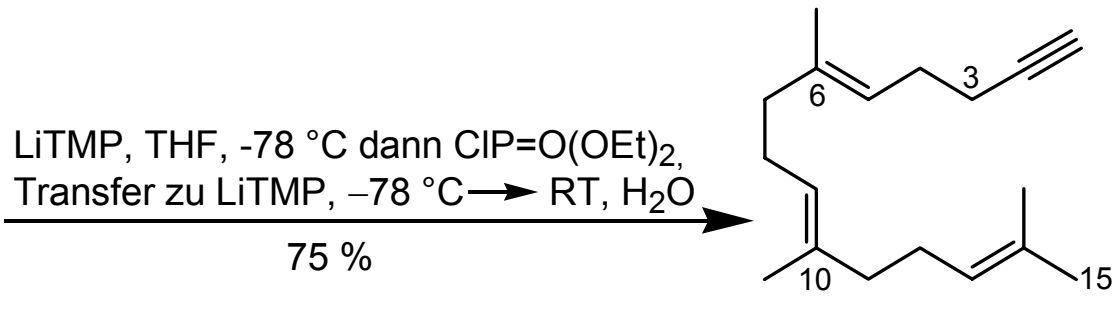

9

In einem ausgeheizten $250 \mathrm{ml}$ Dreihalskolben mit Innenthermometer, $50 \mathrm{ml}$ Tropftrichter und Magnetrührkern wurden in einer Argon-Atmosphäre $2.97 \mathrm{~g}$ (21.0 mmol, 1.05 Äquiv.) 2,2,6,6Tetramethylpiperidin in $20 \mathrm{ml}$ Tetrahydrofuran (K) gelöst und auf $0{ }^{\circ} \mathrm{C}$ abgekühlt. Mit einer Spritze fügte man langsam $15.44 \mathrm{ml}$ (21.0 mmol, 1.05 Äquiv.) einer $1.6 \mathrm{M} \mathrm{n}$-ButyllithiumLösung in $\operatorname{Hexan}^{[113]} \mathrm{zu}$, rührte $30 \mathrm{~min}$ bei $0{ }^{\circ} \mathrm{C}$ und kühlte dann mit einem Aceton/Trockeneis-Bad auf $-78{ }^{\circ} \mathrm{C}$ ab. Aus dem Tropftrichter ließ man eine Lösung von $5.25 \mathrm{~g}$ (20.0 mmol, 1.0 Äquiv.) (E,E)-Farnesylaceton (10) in $15 \mathrm{ml}$ Tetrahydrofuran (K) zur Reaktionsmischung tropfen und rührte $1 \mathrm{~h}$ bei $-78^{\circ} \mathrm{C}$. Dann spritzte man $3.62 \mathrm{~g}(21.0 \mathrm{mmol}$, 1.05 Äquiv.) Diethylchlorophosphat zu, entfernte das Kühlbad, ließ den Ansatz auf Raumtemperatur auftauen und rührte bei dieser Temperatur weitere $3 \mathrm{~h}$ (Mischung A).

In einem separaten, trockenen $250 \mathrm{ml}$ Dreihalskolben mit Innenthermometer, $100 \mathrm{ml}$ Tropftrichter und Magnetrührkern wurden in einer Argon-Atmosphäre $6.36 \mathrm{~g}$ (45 mmol, 2.25 Äquiv.) 2,2,6,6-Tetramethylpiperidin unter Rühren in $30 \mathrm{ml}$ Tetrahydrofuran (K) gelöst, durch Zufügen von $33.10 \mathrm{ml}$ (45 mmol, 2.25 Äquiv.) einer 1.6 $M n$-Butyllithium Lösung in Hexan bei $0{ }^{\circ} \mathrm{C}$ deprotoniert und anschließend auf $-78^{\circ} \mathrm{C}$ abgekühlt (Mischung B).

Zur Vereinigung beider Reaktionsmischungen wurde zunächst Reaktionsmischung A unter Ausschluß von Luftfeuchtigkeit mit Hilfe einer an beiden Enden spitzen Kanüle durch leichten Argon-Überdruck in den $100 \mathrm{ml}$ Tropftrichter von Reaktionsmischung B übergeführt. Dann tropfte man Mischung A während 45 min langsam unter Rühren in die $-78{ }^{\circ} \mathrm{C}$ kalte Mischung B, entfernte das Kühlbad, ließ den Ansatz auf Raumtemperatur auftauen und 2 - $3 \mathrm{~h}$ rühren. Zum Schluß kühlte man auf $0{ }^{\circ} \mathrm{C}$ ab und quenchte die Reaktion durch Zugabe von $30 \mathrm{ml}$ Wasser. 
Zur Aufarbeitung wurde die organische Phase abgetrennt, die wäßrige Phase mit Natriumchlorid gesättigt und dreimal mit je $50 \mathrm{ml}$ Pentan extrahiert. Die organischen Phasen wurden vereinigt und je einmal mit $20 \mathrm{ml}$ eiskalter $1 M$ Salzsäure, $20 \mathrm{ml}$ Wasser und $20 \mathrm{ml}$ gesättigter Natriumhydrogencarbonatlösung gewaschen $(\mathrm{pH}>8)$. Hierbei entstand nach der Extraktion mit Salzsäure, beim Zufügen von Wasser zur organischen Phase eine Emulsion, die sich jedoch nach Zugabe der gesättigten Natriumhydrogencarbonatlösung auflöste und zwei gut trennbare Phasen ergab. Die organischen Phasen wurden über Natriumsulfat getrocknet und das Lösungsmittel i. Vak. am Rotationsverdampfer evaporiert. Zur Reinigung wurde das Rohprodukt an $400 \mathrm{~g}$ Kieselgel chromatographiert $\left(\mathrm{PE} / \mathrm{CH}_{2} \mathrm{Cl}_{2}=100\right.$ : 1). Es wurden 3.67 g (75 \% Ausbeute) des Alkins (9) als klares Öl isoliert.

$\mathbf{R}_{\mathbf{f}}=0.31\left(\mathrm{PE} / \mathrm{CH}_{2} \mathrm{Cl}_{2}=100: 1\right)$.

IR (Film): $\widetilde{v}=3311 \mathrm{~cm}^{-1}(\equiv \mathrm{C}-\mathrm{H}), 2966,2921,2854\left(\mathrm{CH}_{2}, \mathrm{CH}_{3}\right), 2119(\mathrm{C} \equiv \mathrm{C}), 1667(\mathrm{C}=\mathrm{C})$, 1445, $1382\left(\mathrm{CH}_{2}, \mathrm{CH}_{3}\right), 836(=\mathrm{C}-\mathrm{H}), 630(\equiv \mathrm{C}-\mathrm{H})$.

UV $\left(\mathrm{CH}_{3} \mathrm{CN}\right)$ : Die Verbindung 9 zeigt im Wellenlängenbereich von $\lambda=190-400 \mathrm{~nm}$ keine UV-Absorption.

${ }^{1}$ H NMR $\left(300 \mathrm{MHz}, \mathrm{C}_{6} \mathrm{D}_{6}\right): \delta=1.48\left(\mathrm{~s}, 3 \mathrm{H}, 14-\mathrm{CH}_{3}\right), 1.55$ (s, 3H, 6-CH 3 bzw. 10-CH $), 1.57$ $\left(\mathrm{s}, 3 \mathrm{H}, 10-\mathrm{CH}_{3}\right.$ bzw. 6- $\left.\mathrm{CH}_{3}\right), 1.66\left(\mathrm{~d},{ }^{4} J_{15 \mathrm{H}, 13 \mathrm{H}}=1.0 \mathrm{~Hz}, 3 \mathrm{H}, 15-\mathrm{H}_{3}\right), 1.77\left(\mathrm{t},{ }^{4} J_{1 \mathrm{H}, 3 \mathrm{H}_{\mathrm{A}}}=\right.$ $\left.{ }^{4} J_{1 \mathrm{H}, 3 \mathrm{H}_{\mathrm{B}}}=2.5 \mathrm{~Hz}, 1 \mathrm{H}, 1-\mathrm{H}\right), 1.96-2.22\left(\mathrm{~m}, 12 \mathrm{H}, 3-\mathrm{H}_{2}, 4-\mathrm{H}_{2}, 7-\mathrm{H}_{2}, 8-\mathrm{H}_{2}, 11-\mathrm{H}_{2}, 12-\mathrm{H}_{2}\right), 5.16-$ $5.28(\mathrm{~m}, 3 \mathrm{H}, 5-\mathrm{H}, 9-\mathrm{H}, 13-\mathrm{H})$.

${ }^{13} \mathbf{C}$ NMR $\left(50 \mathrm{MHz}, \mathrm{C}_{6} \mathrm{D}_{6}\right): \delta={ }^{2}+16.08\left(6-\mathrm{CH}_{3}\right.$ und $\left.10-\mathrm{CH}_{3}\right),{ }^{\prime}+17.72\left(14-\mathrm{CH}_{3}\right),{ }^{\prime}-{ }^{\prime} 19.17$ (C-3), '+' 25.84 (C-15), '-' 26.90 (C-4), '-' 27.20 (C-8 oder C-12), '-’ 27.51 (C-12 oder C8), '-' 40.00 (C-7 oder C-11), '-' 40.16 (C-11 oder C-7), '+' 68.81 (C-1), '-' 84.27 (C-2), '+' 123.09 (C-5), '+' 124.63 (C-9 oder C-13), '+' 124.91 (C-13 oder C-9), '-' 131.09 (C-14), '-' 135.01 (C-6 oder C-10), '-' 136.49 (C-10 oder C-6).

MS $\left(200 \mathrm{eV}, \mathrm{DCI} / \mathrm{NH}_{3}\right): m / z(\%)=262(100)\left[\mathrm{M}+\mathrm{NH}_{4}{ }^{+}\right], 245(18)\left[\mathrm{M}+\mathrm{H}^{+}\right]$.

$\mathrm{C}_{18} \mathrm{H}_{28}$ (244.22)

Ber.: C $88.20 \quad$ H 11.48

Gef.: C $88.45 \quad$ H 11.55 


\section{$6.2(1 E, 5 E, 9 E)-1-I o d o-2,6,10,14-$ tetramethyl-pentadeca-1,5,9,13-tetraen $(7)^{[96,103]}$}

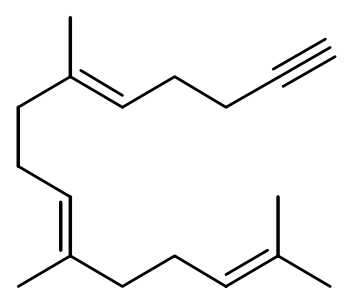

9
1. $\mathrm{Cp}_{2} \mathrm{ZrCl}_{2}, \mathrm{Me}_{3} \mathrm{Al}, \mathrm{CH}_{2} \mathrm{ClCH}_{2} \mathrm{Cl}, \mathrm{RT}$ 2. $\mathrm{I}_{2}, \mathrm{THF},-40{ }^{\circ} \mathrm{C} \rightarrow 0^{\circ} \mathrm{C}$ $74 \%$<smiles>CC=C(C)CCC=C(C)[35F]</smiles>

7

In einem $250 \mathrm{ml}$ Zweihalskolben mit Tieftemperaturthermometer löste man $290 \mathrm{mg}$ (1.0 mmol, 0.25 Äquiv.) Dichlorobis[ $\eta^{5}$-cyclopentadienyl]zirconium in $30 \mathrm{ml}$ trockenem 1,2 Dichlorethan $\left(\mathrm{P}_{4} \mathrm{O}_{10}\right)$, tropfte bei Raumtemperatur $6.0 \mathrm{ml}$ (12.0 mmol, 3.0 Äquiv.) einer $2 \mathrm{M}$ Lösung von Trimethylaluminium in Heptan zu und rührte $15 \mathrm{~min}$. Dann kühlte man auf $0{ }^{\circ} \mathrm{C}$ ab, spritzte eine Lösung von $980 \mathrm{mg}$ (4.0 mmol, 1.0 Äquiv.) des Alkins 9 in $6 \mathrm{ml} \mathrm{1,2}$ Dichlorethan $\left(\mathrm{P}_{4} \mathrm{O}_{10}\right)$ hinzu und rührte noch $30 \mathrm{~min}$ in der Kälte, ehe die Reaktionsmischung auf Raumtemperatur erwärmt wurde. Der Reaktionskolben wurde mit Aluminiumfolie verkleidet und $20 \mathrm{~h}$ bei Raumtemperatur gerührt, dann auf -40 bis $-30{ }^{\circ} \mathrm{C}$ abgekühlt und mit einer Lösung von $1.22 \mathrm{~g}$ (4.8 mmol, 1.2 Äquiv.) Iod in Tetrahydrofuran gequencht. Nach 20 min erwärmte man mit einer Eis/Kochsalz-Kältemischung die Reaktionsmischung auf $-10{ }^{\circ} \mathrm{C}$ und spritzte $2 \mathrm{ml}$ einer gesättigten Kaliumcarbonatlösung so $\mathrm{zu}$, daß die Temperatur im Kolbeninneren nicht über $5{ }^{\circ} \mathrm{C}$ stieg. Nach 15 min wurden $8 \mathrm{~g}$ einer Mischung aus gleichen Anteilen Magnesiumsulfat und Natriumsulfat in das Reaktiongefäß gegeben und $1 \mathrm{~h}$ bei Raumtemperatur kräftig gerührt. Zum Abtrennen der festen Bestandteile filtrierte man über eine dünne Schicht aus $3 \mathrm{~g}$ Celite und spülte mit genügend Diethylether nach. Die Lösungsmittel wurden am Rotationsverdampfer i. Vak. entfernt und das rohe Vinyliodid an 500 g Kieselgel mit reinem Petrolether als Laufmittel chromatographiert. Es wurden $1.14 \mathrm{~g}$ (74 \% Ausbeute) des Vinyliodids 7 als klares Öl isoliert.

$\mathbf{R}_{\mathbf{f}}=0.35(\mathrm{PE})$.

IR (Film): $\widetilde{v}=3054 \mathrm{~cm}^{-1}(=\mathrm{C}-\mathrm{H}), 2966,2923,2854\left(\mathrm{CH}_{2}, \mathrm{CH}_{3}\right), 1714,1666,1618(\mathrm{C}=\mathrm{C})$, 1446, 1378, $\left(\mathrm{CH}_{2}, \mathrm{CH}_{3}\right), 1368\left(\mathrm{CH}_{3}\right)$.

UV $\left(\mathrm{CH}_{3} \mathrm{CN}\right)$ : Die Verbindung 7 zeigt im Wellenlängenbereich $\lambda=190$ bis $400 \mathrm{~nm}$ keine UV-Absorption. 
${ }^{1} \mathbf{H}$ NMR $\left(300 \mathrm{MHz}, \mathrm{C}_{6} \mathrm{D}_{6}\right): \delta=1.49\left(\mathrm{~s}, 3 \mathrm{H}, 6-\mathrm{CH}_{3}\right.$ bzw. 10- $\left.\mathrm{CH}_{3}\right), 1.56\left(\mathrm{~s}, 3 \mathrm{H}, 14-\mathrm{CH}_{3}\right), 1.60$ (s, 3H, 10- $\mathrm{CH}_{3}$ bzw. 6- $\left.\mathrm{CH}_{3}\right), 1.66\left(\mathrm{~s}, 3 \mathrm{H}, 2-\mathrm{CH}_{3}\right), 1.67\left(\mathrm{~s}, 3 \mathrm{H}, 15-\mathrm{H}_{3}\right), 1.92\left(\mathrm{~m}_{\mathrm{C}}, 4 \mathrm{H}, 3-\mathrm{H}_{2}, 4-\right.$ $\left.\mathrm{H}_{2}\right), 2.00-2.22\left(\mathrm{~m}, 8 \mathrm{H}, 7-\mathrm{H}_{2}, 8-\mathrm{H}_{2}, 11-\mathrm{H}_{2}, 12-\mathrm{H}_{2}\right) 5.04\left(\mathrm{~m}_{\mathrm{C}}, 1 \mathrm{H}, 13-\mathrm{H}\right), 5.22$ - $5.27(\mathrm{~m}, 2 \mathrm{H}$, $5-\mathrm{H}, 9-\mathrm{H}), 5.71\left(\mathrm{~d},{ }^{4} \mathrm{~J}=0.7 \mathrm{~Hz}, 1 \mathrm{H}, 1-\mathrm{H}\right)$.

${ }^{13} \mathrm{C}$ NMR $\left(50 \mathrm{MHz}, \mathrm{C}_{6} \mathrm{D}_{6}\right): \delta={ }^{+}{ }^{\prime} 16.08\left(6-\mathrm{CH}_{3}\right.$ oder $\left.10-\mathrm{CH}_{3}\right), 16.19\left(10-\mathrm{CH}_{3}\right.$ oder $\left.6-\mathrm{CH}_{3}\right)$, '+' $17.80\left(14-\mathrm{CH}_{3}\right)$, ’+' $23.92\left(2-\mathrm{CH}_{3}\right)$, ’+' $25.90(\mathrm{C}-15)$, '-' $26.54(\mathrm{C}-4)$, ’-' $27.09(\mathrm{C}-8$ oder C-12), '-' 27.27 (C-12 oder C-8), ’-' 39.54 (C-3), '-' 40.10 (C-7 oder C-11), ’-' 40.24 (C-11 oder C-7), '+' 75.39 (C-1), '+' 123.52 (C-5), '+' 124.64 (C-9 oder C-13), '+' 124.90 (C-13 oder C-9), '-' 131.13 (C-14), '-' 135.09 (C-6 oder C-10), '-' 135.92 (C-10 oder C-6), '-' $147.61(\mathrm{C}-2)$.

MS $(70 \mathrm{eV}): m / z(\%)=386(6)\left[\mathrm{M}^{+}\right], 259(11)\left[\mathrm{M}^{+}-\mathrm{I}\right], 191(5)\left[259-\mathrm{C}_{5} \mathrm{H}_{8}\right], 136(36)$ $\left[\mathrm{C}_{10} \mathrm{H}_{16}{ }^{+}\right], 121(24)\left[\mathrm{C}_{9} \mathrm{H}_{13}{ }^{+}\right], 69(100)\left[\mathrm{C}_{5} \mathrm{H}_{9}^{+}\right], 41(20)\left[\mathrm{C}_{3} \mathrm{H}_{5}{ }^{+}\right]$.

$\mathbf{C}_{\mathbf{1 9}} \mathbf{H}_{31} \mathbf{I}(\mathbf{3 8 6 . 1 5 )} \quad$ Ber.: C $59.04 \quad$ H 8.09

Gef.: C 58.77 H 7.90 


\section{Synthese von racemischem Methanophenazin (rac-1)}

\section{$7.1 \quad(6 E, 10 E, 14 E)-(3 R S)-( \pm)-1-t e r t$. -Butyldimethylsilyloxy-(3,7,11,15,19-pentamethyl- eicosa-6,10,14,18-tetraenyloxy)-silan $\left(\right.$ rac-76) ${ }^{[105]}$}

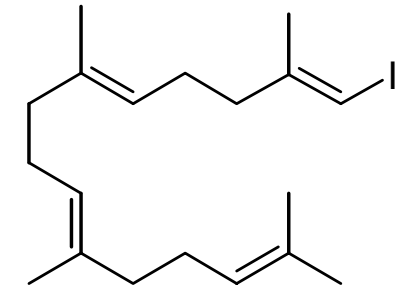

7 rac-6, $\mathrm{ZnCl}_{2}, \mathrm{Et}_{2} \mathrm{O}, \mathrm{RT}$, dann tert.-BuLi, $-90^{\circ} \mathrm{C} \rightarrow \mathrm{RT}$, Transfer $\frac{\text { zu 7, kat. } \mathrm{Pd}\left(\mathrm{PPh}_{3}\right)_{4}, \mathrm{RT}}{65 \%}$<smiles>CC(C)=CCCC(C)=CCCC(C)CCOC(C)(C)C</smiles>

rac-76

Es wurden $204 \mathrm{mg}$ (1.5 mmol, 1.5 Äquiv.) Zinkchlorid mit einer Heißluftpistole i. Vak. geschmolzen und in einer Argon-Atmosphäre abgekühlt. Anschließend versetzt man das trockene Zinkchlorid mit einer Lösung aus $513 \mathrm{mg}$ (1.5 mmol, 1.5 Äquiv.) des Alkyliodids rac-6 in $12 \mathrm{ml}$ Diethylether $(\mathrm{Na})$ und rührt bei Raumtemperatur ca. $1 \mathrm{~h}$ bis sich das Zinkchlorid vollständig gelöst hatte. Eine Kältemischung, bestehend aus Petrolether/Isopropanol/Aceton im Verhältnis $4: 1: 1$, wurde mit flüssigem Stickstoff auf $-100{ }^{\circ} \mathrm{C}$ gebracht und die Reaktionsmischung darin abgekühlt. Bei dieser Temperatur tropfte man $3 \mathrm{ml}$ (4.5 mmol, 4.5 Äquiv.) einer 1.5 M Lösung von tert.-Butylithium in Pentan aus einer Spritze langsam zu, rührte noch 30 min in der Kälte, entfernte dann das Kältebad und ließ die Reaktionsmischung auftauen. Nach $1 \mathrm{~h}$ Rühren bei Raumtemperatur wurde der Ansatz unter Ausschluß von Luftfeuchtigkeit, durch eine an beiden Seiten spitze Kanüle, unter einem leichten Überdruck von Argon in einen separaten Kolben übergeführt, der 386 $\mathrm{mg}$ (1.0 mmol, 1.0 Äquiv.) Vinyliodid 7 und $60 \mathrm{mg}$ (0.09 mmol, 0.09 Äquiv.) Tetrakistriphenylphosphinpalladium enthielt. Nach $10 \mathrm{~h}$ Rühren bei Raumtemperatur wurde die Reaktionsmischung zur Aufarbeitung in $30 \mathrm{ml}$ Diethylether aufgenommen, zweimal mit je $15 \mathrm{ml}$ gesättigter Natriumchloridlösung gewaschen, über Natriumsulfat getrocknet, filtriert, und das Solvens am Rotationsverdampfer i. Vak. entfernt. Das Rohprodukt wurde an $50 \mathrm{~g}$ Kieselgel $\left(\mathrm{PE}_{\mathrm{Et}} \mathrm{O}=100: 1\right)$ chromatographiert. Es wurden $307 \mathrm{mg}$ (65\% Ausbeute bezogen auf das Vinyliodid 7) der Titelverbindung rac-76 als klares farbloses Öl isoliert. 
$\mathbf{R}_{\mathbf{f}}=0.47\left(\mathrm{Et}_{2} \mathrm{O} / \mathrm{PE}=1: 100\right)$.

IR (Film): $\widetilde{v}=2956,2927,2856 \mathrm{~cm}^{-1}\left(\mathrm{CH}_{2}, \mathrm{CH}_{3}\right), 1665,1618(\mathrm{C}=\mathrm{C}), 1471,1461,1449$, 1383, $\left(\mathrm{CH}_{2}, \mathrm{CH}_{3}\right), 1254$ (C-OSi), 1096, 836 (Si-OC), $775\left(\mathrm{C}-\mathrm{SiCH}_{3}\right)$.

$\mathbf{U V}\left(\mathrm{CH}_{3} \mathrm{CN}\right): \lambda_{\max }(\lg \varepsilon)=248(2.41)$.

${ }^{1} \mathbf{H}$ NMR $\left(500 \mathrm{MHz}, \mathrm{C}_{6} \mathrm{D}_{6}\right): \delta=0.09\left[\mathrm{~s}, 6 \mathrm{H}, \mathrm{Si}\left(\mathrm{CH}_{3}\right)_{2}\right], 0.92\left[\mathrm{~d},{ }^{3} J_{3 \mathrm{CH}_{3}, 3 \mathrm{H}}=6.8 \mathrm{~Hz}, 3 \mathrm{H}, 3-\right.$ $\left.\left.\mathrm{CH}_{3}\right)\right], 1.00\left[\mathrm{~s}, 9 \mathrm{H}, \mathrm{SiC}\left(\mathrm{CH}_{3}\right)_{3}\right], 1.23\left(\mathrm{~m}_{\mathrm{C}}, 1 \mathrm{H}, 4-\mathrm{H}_{\mathrm{B}}\right), 1.32-1.48\left(\mathrm{~m}, 2 \mathrm{H}, 2-\mathrm{H}_{\mathrm{A}}, 4-\mathrm{H}_{\mathrm{A}}\right), 1.57(\mathrm{~s}$, $\left.3 \mathrm{H}, 19-\mathrm{CH}_{3}\right), 1.63-1.69(\mathrm{~m}, 1 \mathrm{H}, 3-\mathrm{H})$ überlagert von $1.62\left(\mathrm{~s}, 6 \mathrm{H}, 7-\mathrm{CH}_{3}, 11-\mathrm{CH}_{3}\right)$ und 1.64 $\left(\mathrm{s}, 3 \mathrm{H}, 15-\mathrm{CH}_{3}\right), 1.69\left(\mathrm{~d},{ }^{4} J_{20 \mathrm{H}, 18 \mathrm{H}}=1.0 \mathrm{~Hz}, 3 \mathrm{H}, 20-\mathrm{H}_{3}\right), 2.01-2.26\left(\mathrm{~m}, 15 \mathrm{H}, 2-\mathrm{H}_{\mathrm{B}}, 5-\mathrm{H}_{2}, 8-\right.$ $\left.\mathrm{H}_{2}, 9-\mathrm{H}_{2}, 12-\mathrm{H}_{2}, 13-\mathrm{H}_{2}, 16-\mathrm{H}_{2}, 17-\mathrm{H}_{2}\right), 3.64\left(\mathrm{~m}_{\mathrm{C}}, 2 \mathrm{H}, 1-\mathrm{H}_{2}\right), 5.25$ (t br, ${ }^{3} J_{18 \mathrm{H}, 17 \mathrm{H}_{2}}=7.0 \mathrm{~Hz}$, aufgespalten durch Fernkopplungen $\left.{ }^{4} J \approx 1.2 \mathrm{~Hz}, 1 \mathrm{H}, 18-\mathrm{H}\right), 5.27$ - 5.35 (m, 3H, 6-H, 10-H, 14-H).

${ }^{13} \mathrm{C}$ NMR $\left(50 \mathrm{MHz}, \mathrm{CDCl}_{3}\right): \delta={ }^{+}{ }^{\prime}-5.23\left[\mathrm{Si}\left(\mathrm{CH}_{3}\right)_{2}\right]$, ' +' 15.99 und '+' $16.03\left(7-\mathrm{CH}_{3}, 11-\right.$ $\left.\mathrm{CH}_{3}, 15-\mathrm{CH}_{3}\right)$, `' $17.70\left(19-\mathrm{CH}_{3}\right)$, ’-' $18.35\left[\mathrm{SiC}\left(\mathrm{CH}_{3}\right)_{3}\right]$, ’+' $19.66\left(3-\mathrm{CH}_{3}\right)$, ’-' $25.41(\mathrm{C}-5)$, '+' $25.72(\mathrm{C}-20)$, '+' $25.99\left[\mathrm{SiC}\left(\mathrm{CH}_{3}\right)_{3}\right]$ '-' 26.68 und '-' $26.78(\mathrm{C}-9, \mathrm{C}-13, \mathrm{C}-17)$, '+' 29.22 (C-3), '-' 37.25 (C-2 und C-4), '-' 39.73 und '-' 39.94 (C-8, C-12, C-16), '-' 61.48 (C-1), '+'124.26, '+' 124.39, '+' 124.71, (C-6, C-10, C-14, C-18), '-' 131.21 (C-19), '-' 134.68, '-' 134.86 (C-7, C-11, C-15).

MS $(70 \mathrm{eV}): m / z(\%)=474(18)\left[\mathrm{M}^{+}\right], 417(16)\left[\mathrm{M}^{+}-\mathrm{C}_{4} \mathrm{H}_{9}\right], 405(10)\left[\mathrm{M}^{+}-\mathrm{C}_{5} \mathrm{H}_{9}\right], 335(4)$ [405- $\left.\mathrm{C}_{5} \mathrm{H}_{10}\right], 204(28)\left[\mathrm{C}_{15} \mathrm{H}_{24}{ }^{+}\right], 123(23)\left[204-\mathrm{C}_{5} \mathrm{H}_{8}\right], 81(64)\left[\mathrm{C}_{6} \mathrm{H}_{9}{ }^{+}\right], 69$ (100) $\left[\mathrm{C}_{5} \mathrm{H}_{9}{ }^{+}\right], 41$ (14) $\left[\mathrm{C}_{3} \mathrm{H}_{5}^{+}\right]$.

MS $\left(200 \mathrm{eV}, \mathrm{DCI} / \mathrm{NH}_{3}\right): m / z(\%)=492(100)\left[\mathrm{M}+\mathrm{NH}_{4}{ }^{+}\right]$.

HR-MS: ber. für $\mathrm{C}_{31} \mathrm{H}_{58} \mathrm{SiO}$ : 474.4247; gef.: 474.4256 .

\section{$\mathrm{C}_{31} \mathrm{H}_{58} \mathrm{SiO}(474.43)$}


$7.2(6 E, 10 E, 14 E)-(3 R S)-3,7,11,15,19-P e n t a m e t h y l-e i c o s a-6,10,14,18-t e t r a e n-1-o l ~(r a c-78)$

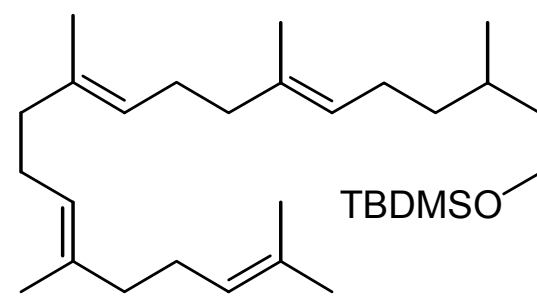

rac-76
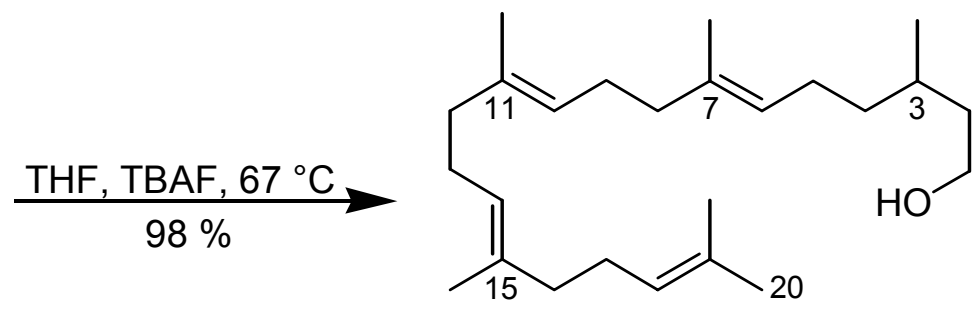

rac-78

$236.5 \mathrm{mg}$ (0.5 mmol, 1.0 Äquiv.) des Silylethers rac-76 wurden in $4 \mathrm{ml}$ einer 0.5 M Lösung von Tetrabutylammoniumfluorid-Trihydrat (2 mmol Fluorid, 4.0 Äquiv.) in Tetrahydrofuran gelöst und zum Sieden erhitzt. Nach ca. 30 min war das Substrat laut dünnschichtchromatographischer Analyse der Reaktionsmischung vollständig desilyliert $\left(\mathrm{Et}_{2} \mathrm{O} / \mathrm{Pentan}=1: 1\right)$. Zur Reaktionsmischung wurde $1 \mathrm{~g}$ Kieselgel zugegeben und das Solvens am Rotationsverdampfer i. Vak. entfernt. Das so auf Kieselgel aufgezogene Rohprodukt wurde zur Reinigung an $25 \mathrm{~g}$ Kieselgel chromatographiert $\left(\mathrm{Et}_{2} \mathrm{O} /\right.$ Pentan $\left.=1: 10 \rightarrow 1: 1\right)$. Es wurden $176 \mathrm{mg}(98 \%$ Ausbeute) des Alkohols rac-78 als klares Öl isoliert.

$\mathbf{R}_{\mathbf{f}}=0.47\left(\mathrm{Et}_{2} \mathrm{O} /\right.$ Pentan $\left.=1: 1\right)$.

IR (Film): $\widetilde{v}=3331 \mathrm{~cm}^{-1}(\mathrm{OH}), 2960,2924,2854\left(\mathrm{CH}_{2}, \mathrm{CH}_{3}\right), 1666,(\mathrm{C}=\mathrm{C}), 1450,1379$ $\left(\mathrm{CH}_{2}, \mathrm{CH}_{3}\right), 1057$ (C-O).

UV $\left(\mathrm{CH}_{3} \mathrm{CN}\right)$ : Die Verbindung rac-78 zeigt im Wellenlängenbereich von $\lambda=190-400 \mathrm{~nm}$ keine UV-Absorption.

${ }^{1} \mathbf{H}$ NMR $\left(300 \mathrm{MHz}, \mathrm{C}_{6} \mathrm{D}_{6}\right): \delta=0.83\left(\mathrm{~d},{ }^{3} \mathrm{~J}_{3 \mathrm{CH}_{3}, 3 \mathrm{H}}=7.0 \mathrm{~Hz}, 3 \mathrm{H}, 3-\mathrm{CH}_{3}\right), 1.10-1.28(\mathrm{~m}, 2 \mathrm{H}$, 4- $\left.\mathrm{H}_{2}\right), 1.30-1.40\left(\mathrm{~m}, 2-\mathrm{H}_{\mathrm{B}}\right), 1.41-1.50\left(\mathrm{~m}, 1 \mathrm{H}, 2-\mathrm{H}_{\mathrm{A}}\right), 1.50-1.58(\mathrm{~m}, 1 \mathrm{H}, 3-\mathrm{H}), 1.54(\mathrm{~s}, 1 \mathrm{H}$, $\mathrm{OH}), 1.56\left(\mathrm{~s}, 3 \mathrm{H}, 7-\mathrm{CH}_{3}\right), 1.61\left(\mathrm{~s}, 9 \mathrm{H}, 11-\mathrm{CH}_{3}, 15-\mathrm{CH}_{3}, 19-\mathrm{CH}_{3}\right), 1.67\left(\mathrm{~d},{ }^{4} \mathrm{~J}_{20 \mathrm{H}_{3}, 18 \mathrm{H}}=0.7 \mathrm{~Hz}\right.$, $\left.3 \mathrm{H}, 20-\mathrm{H}_{3}\right), 1.95-2.25\left(\mathrm{~m}, 14 \mathrm{H}, 5-\mathrm{H}_{2}, 9-\mathrm{H}_{2}, 8-\mathrm{H}_{2}, 12-\mathrm{H}_{2}, 13-\mathrm{H}_{2}, 16-\mathrm{H}_{2}, 17-\mathrm{H}_{2}\right), 3.40\left(\mathrm{~m}_{\mathrm{C}}\right.$, $\left.2 \mathrm{H}, 1-\mathrm{H}_{2}\right), 5.19-5.28$ (m, 2H, 10-H, 14-H), 5.28 (t br, 2H, 6-H, 18-H).

${ }^{13} \mathbf{C}$ NMR (50 MHz, $\left.\mathrm{C}_{6} \mathrm{D}_{6}\right): \delta={ }^{+}+16.09$ und '+' $16.13\left(7-\mathrm{CH}_{3}, 11-\mathrm{CH}_{3}, 15-\mathrm{CH}_{3}\right)$, ' $+{ }^{\prime} 17.74$ $\left(19-\mathrm{CH}_{3}\right),{ }^{+}+19.70\left(3-\mathrm{CH}_{3}\right)$, '-' $25.85(\mathrm{C}-5)$, '+' $25.88(\mathrm{C}-20)$, '-' $25.90(\mathrm{C}-2)$, '-' 27.10 und '-' 27.23 (C-9, C-13, C-17), '+' 29.49 (C-3), '-' 37.63 (C-4), '-' 40.20 (C-8, C-12, C16), '-' $60.83(\mathrm{C}-1)$, '+'124.80 (C-10 und C-14), '+' 124.93 (C-6), '+' 125.26 (C-18), '-' 131.09 (C-19), '-' 134.78, '-' 134.97, '-' 134.99 (C-7, C-11, C-15).

MS $\left(200 \mathrm{eV}, \mathrm{DCI} / \mathrm{NH}_{3}\right): m / z(\%)=492(100)\left[\mathrm{M}+\mathrm{NH}_{4}^{+}\right]$. 


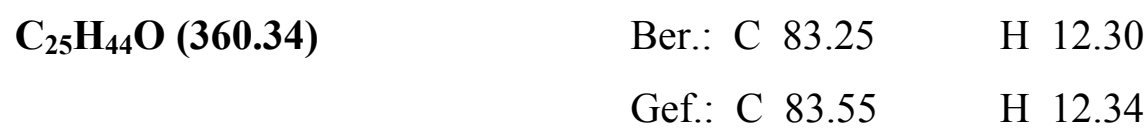

7.3 (E6',E10',E14')-(3'RS)-( $( \pm)-2-(3,7,11,15,19-P e n t a m e t h y l-e i c o s a-6,10,14,18-$ tetraenyloxy)-phenazin (rac-1)
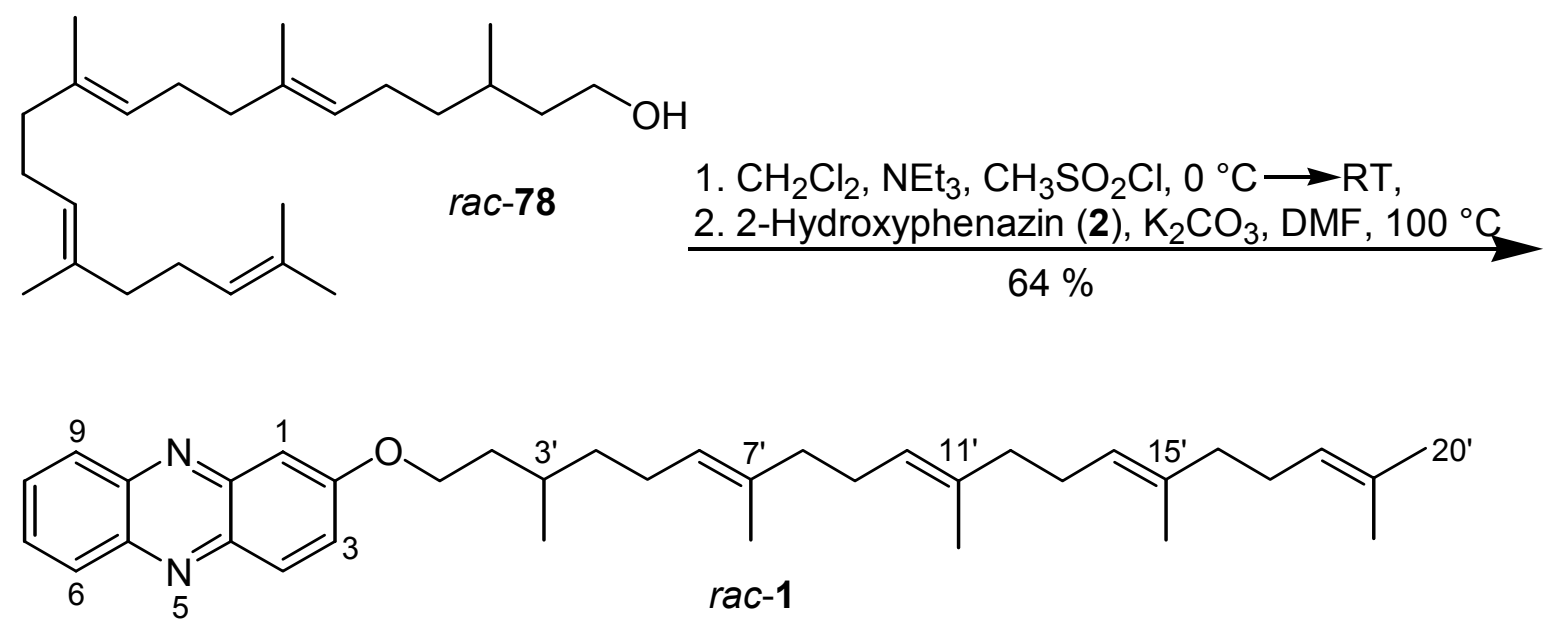

Es wurden bei $0{ }^{\circ} \mathrm{C} 90 \mathrm{mg}(0.25 \mathrm{mmol}, 1.0$ Äquiv.) Alkohol rac-78 in $3 \mathrm{ml}$ trockenem Dichlormethan $\left(\mathrm{P}_{4} \mathrm{O}_{10}\right)$ gelöst, mit $55 \mu \mathrm{l}(40 \mathrm{mg}, 0.37 \mathrm{mmol}, 1.47$ Äquiv.) frisch destilliertem Triethylamin und mit $32 \mathrm{mg}$ (0.28 mmol, 1.1 Äquiv.) Methansulfonsäurechlorid versetzt. Nach ca. 30 min war das Substrat vollständig umgesetzt (DC-Kontrolle: $\mathrm{Et}_{2} \mathrm{O} / \mathrm{PE}=1: 2$ ). Der Ansatz wurde in $3 \mathrm{ml}$ ges. Natriumhydrogencarbonatlösung aufgenommen und viermal mit je $10 \mathrm{ml}$ Diethylether extrahiert. Die organischen Phasen wurden vereinigt, über Natriumsulfat getrocknet, filtriert und i. Vak. vom Lösungsmittel befreit. Das rohe Mesylat wurde anschließend in $5 \mathrm{ml}$ trockenem Dimethylformamid $\left(\mathrm{CaH}_{2}\right)$ gelöst, mit $172 \mathrm{mg}(1.25 \mathrm{mmol}$, 5.0 Äquiv.) wasserfreiem, fein gemörsertem Kaliumcarbonat und $55 \mathrm{mg}$ (0.28 mmol, 1.1 Äquiv.) 2-Hydroxyphenazin (2) versetzt. Diese Reaktionsmischung wurde für $10 \mathrm{~h}$ auf 100 ${ }^{\circ} \mathrm{C}$ erhitzt (DC Kontrolle: $\mathrm{CH}_{2} \mathrm{Cl}_{2} / \mathrm{PE}=100: 1$ ) und nach dem Abkühlen mit $5 \mathrm{ml}$ Wasser verdünnt. Nun extrahierte man die Reaktionsmischung einmal mit $10 \mathrm{ml}$ Diethylether, trennte die wäßrige Phase ab, sättigte sie mit Natriumchlorid und extrahierte erneut dreimal mit je 5 $\mathrm{ml}$ Diethylether. Die organischen Phasen wurden vereinigt und einmal mit $10 \mathrm{ml}$ gesättigter Ammoniumchloridlösung gewaschen, über Natriumsulfat getrocknet, filtriert und i. Vak. das Solvens am Rotationsverdampfer entfernt. Zur Reinigung wurde das Rohprodukt an $20 \mathrm{~g}$ 
Kieselgel unter Druck chromatographiert $\left(\mathrm{CH}_{2} \mathrm{Cl}_{2} / \mathrm{PE}=100: 1\right)$. Es wurden $86 \mathrm{mg}$ (64\% Ausbeute bezogen auf den Alkohol rac-78) des Naturstoffs $r a c-1$ als gelbes Öl isoliert.

$\mathbf{R}_{\mathbf{f}}=0.51$ (Diethylether/Hexan $\left.=1: 1\right)$.

IR (Film): $\widetilde{v}=3059 \mathrm{~cm}^{-1}(=\mathrm{C}-\mathrm{H}), 2954,2924,2853\left(\mathrm{CH}_{2}, \mathrm{CH}_{3}\right.$.), 1632, 1605, 1560, 1518 $(\mathrm{C}=\mathrm{C})$, 1483, 1445 1380, $1360\left(\mathrm{CH}_{2}, \mathrm{CH}_{3}\right), 1196(\mathrm{C}-\mathrm{O}-\mathrm{C}), 828,757(\mathrm{C}=\mathrm{C}$, arom.).

UV $\left(\mathrm{CH}_{3} \mathrm{CN}\right): \lambda_{\max }(\lg \varepsilon)=388$ (3.72), 355 (3.78), $256 \mathrm{~nm}$ (4.71).

${ }^{1} \mathbf{H}$ NMR $\left(200 \mathrm{MHz}, \mathrm{C}_{6} \mathrm{D}_{6}\right): \delta=0.89\left(\mathrm{~d},{ }^{3} J_{3}{ }^{\prime} \mathrm{CH}_{3}, \mathrm{CH}=6.5 \mathrm{~Hz}, 3 \mathrm{H}, 3^{\prime}-\mathrm{CH}_{3}\right), 1.12-1.58(\mathrm{~m}, 4 \mathrm{H}$, 4'- $\left.\mathrm{H}_{2}, 2^{\prime}-\mathrm{H}_{2}\right), 1.61\left(\mathrm{~s}, 3 \mathrm{H}, 19\right.$ '- $\left.\mathrm{H}_{3}\right), 1.70-1.80$ (m, 1H, 3'-H) überlagert von $1.64-1.71(3 \mathrm{~s}, \mathrm{je}$ $\left.3 \mathrm{H}, 7^{\prime}-\mathrm{CH}_{3}, 11^{\prime}-\mathrm{CH}_{3}, 15^{\prime}-\mathrm{CH}_{3}\right)$ und $1.73\left(\mathrm{~s}, 3 \mathrm{H}, 20^{\prime}-\mathrm{H}_{3}\right), 2.02-2.29$ (m, $14 \mathrm{H}, 5^{\prime}-\mathrm{H}_{2}, 8^{\prime}-\mathrm{H}_{2}$, $\left.9^{\prime}-\mathrm{H}_{2}, 12^{\prime}-\mathrm{H}_{2}, 13^{\prime}-\mathrm{H}_{2}, 16^{\prime}-\mathrm{H}_{2}, 17^{\prime}-\mathrm{H}_{2}\right), 3.83\left(\mathrm{t},{ }^{3} J_{1}{ }^{\prime} \mathrm{H}_{2}, 2^{\prime} \mathrm{H}_{2}=6.5,2 \mathrm{H}, 1^{\prime}-\mathrm{H}_{2}\right), 5.21-5.48(\mathrm{~m}$, $\left.4 \mathrm{H}, 6^{\prime}-\mathrm{H}, 10^{\prime}-\mathrm{H}, 14^{\prime}-\mathrm{H}, 18^{\prime}-\mathrm{H}\right), 7.27-7.47$ (m, 3H, 3-H, 7-H, 8-H), $7.56\left(\mathrm{~d},{ }^{4} J_{1 \mathrm{H}, 3 \mathrm{H}}=3.0 \mathrm{~Hz}\right.$, $1 \mathrm{H}, 1-\mathrm{H}), 8.15\left(\mathrm{~d},{ }^{3} J_{4 \mathrm{H}, 3 \mathrm{H}}=9.6 \mathrm{~Hz}, 1 \mathrm{H}, 4-\mathrm{H}\right), 8.34\left(\mathrm{dd},{ }^{3} J_{6 \mathrm{H}, 7 \mathrm{H}}=7.0 \mathrm{~Hz},{ }^{4} J_{6 \mathrm{H}, 8 \mathrm{H}}=2.5 \mathrm{~Hz}, 1 \mathrm{H}\right.$, $6-\mathrm{H}$ und dd, $\left.{ }^{3} J_{9 \mathrm{H}, 8 \mathrm{H}}=7.0 \mathrm{~Hz},{ }^{4} J_{9 \mathrm{H}, 7 \mathrm{H}}=2.5 \mathrm{~Hz}, 1 \mathrm{H}, 9-\mathrm{H}\right)$.

${ }^{1} \mathbf{H}$ NMR $\left(500 \mathrm{MHz}, \mathrm{C}_{6} \mathrm{D}_{6}\right): \delta=0.81\left(\mathrm{~d},{ }^{3} \mathrm{~J}_{3}{ }^{\prime} \mathrm{CH}_{3}, 3^{\prime} \mathrm{H}=6.6 \mathrm{~Hz}, 3 \mathrm{H}, 3^{\prime}-\mathrm{CH}_{3}\right), 1.17\left(\mathrm{~m}_{\mathrm{C}}, 1 \mathrm{H}, 4^{\prime}-\right.$ $\left.\mathrm{H}_{\mathrm{B}}\right), 1.30-1.44\left(\mathrm{~m}, 2 \mathrm{H}, 2^{\prime}-\mathrm{H}_{\mathrm{B}}, 4^{\prime}-\mathrm{H}_{\mathrm{A}}\right), 1.53\left(\mathrm{~s}, 3 \mathrm{H}, 19^{\prime}-\mathrm{CH}_{3}\right), 1.54-1.63\left(\mathrm{~m}, 1 \mathrm{H}, 3^{\prime}-\mathrm{H}\right)$ überlagert von $1.58,1.59,1.60\left(3 \mathrm{~s}, \mathrm{je} 3 \mathrm{H}, 7^{\prime}-\mathrm{CH}_{3}, 11^{\prime}-\mathrm{CH}_{3}, 15^{\prime}-\mathrm{CH}_{3}\right), 1.63\left(\mathrm{~d},{ }^{4} J_{20}{ }^{\prime} \mathrm{H}_{3}, 18^{\prime} \mathrm{H}=\right.$ $\left.1.0 \mathrm{~Hz}, 3 \mathrm{H}, 20^{\prime}-\mathrm{H}_{3}\right), 1.69\left(\mathrm{~m}_{\mathrm{C}}, 1 \mathrm{H}, 2^{\prime}-\mathrm{H}_{\mathrm{A}}\right), 1.94-2.23$ (m, 14H, 5'- $\mathrm{H}_{2}, 8^{\prime} \mathrm{H}_{2}, 9^{\prime} \mathrm{H}_{2}$, 12' $^{\prime}-\mathrm{H}_{2}$, $\left.13^{\prime}-\mathrm{H}_{2}, 16^{\prime}-\mathrm{H}_{2}, 17^{\prime}-\mathrm{H}_{2}\right), 3.76\left(\mathrm{~m}_{\mathrm{C}}, 2 \mathrm{H}, 1^{\prime}-\mathrm{H}_{2}\right), 5.20\left(\mathrm{t}\right.$ br, ${ }^{3} J_{18}{ }^{\prime} \mathrm{H}, 17^{\prime} \mathrm{H}_{2}=6.9 \mathrm{~Hz}$, aufgespalten durch Fernkopplungen $\left.{ }^{4} \mathrm{~J} \approx 1.4 \mathrm{~Hz}, 1 \mathrm{H}, 18^{\prime}-\mathrm{H}\right), 5.22$ - 5.32 (m, 3H, 6'-H, 10'-H, 14'-H), 7.22 - $7.35(\mathrm{~m}, 3 \mathrm{H}, 3-\mathrm{H}, 7-\mathrm{H}, 8-\mathrm{H}), 7.48\left(\mathrm{~d},{ }^{4} J_{1 \mathrm{H}, 3 \mathrm{H}}=2.7 \mathrm{~Hz}, 1 \mathrm{H}, 1-\mathrm{H}\right), 8.06\left(\mathrm{~d},{ }^{3} J_{4 \mathrm{H}, 3 \mathrm{H}}=9.4 \mathrm{~Hz}\right.$, $1 \mathrm{H}, 4-\mathrm{H}), 8.24\left(\mathrm{dd},{ }^{3} J_{6 \mathrm{H}, 7 \mathrm{H}}=8.8 \mathrm{~Hz},{ }^{4} J_{6 \mathrm{H}, 8 \mathrm{H}}=1.2 \mathrm{~Hz}, 1 \mathrm{H}, 6-\mathrm{H}\right.$ oder $\left.9-\mathrm{H}\right), 8.245\left(\mathrm{dd},{ }^{3} J_{9 \mathrm{H}, 7 \mathrm{H}}=\right.$ $8.8 \mathrm{~Hz},{ }^{4} J_{9 \mathrm{H}, 7 \mathrm{H}}=1.2 \mathrm{~Hz}, 1 \mathrm{H}, 6-\mathrm{H}$ oder $\left.9-\mathrm{H}\right)$.

${ }^{13} \mathbf{C}$ NMR (50 MHz, $\left.\mathrm{C}_{6} \mathrm{D}_{6}\right): \delta={ }^{\prime}{ }^{\prime} 16.12\left(7^{\prime}-\mathrm{CH}_{3}, 11^{\prime}-\mathrm{CH}_{3}, 15^{\prime}-\mathrm{CH}_{3}\right),{ }^{\prime}+17.72\left(19^{\prime}-\mathrm{CH}_{3}\right)$, '+' $19.56\left(3^{\prime}-\mathrm{CH}_{3}\right)$, '+' $25.76(\mathrm{C}-20$ '), '-' 25.84 (C-5'), '-' 27.10 und '-' 27.21 (C-9', C-13', C-17'), '+' 29.76 (C-3'), '-' 35.99 (C-2'), '-' 37.43 (C-4'), '-' 40.19 und '-' 40.22 (C-8', C12 ', C-16'), ’-' $66.86(\mathrm{C}-1$ '), '+' $105.80(\mathrm{C}-1)$, '+' 124.72, '+' 124.75, '+' 124.91, (C-10', C14 ', C-18'), '+' 125.03 (C-6'), '+' $126.51(\mathrm{C}-3)$, '+' 128.77 (C-7 oder C-8), ’+' 129.58 (C-8 oder C-7), '+' 130.15 (C-6 oder C-9), '+' 130.27 (C-9 oder C-6), '-' 131.08 (C-19'), '+' 131.30 (C-4), '-' 134.98, '-' 135.01, '-' 135.07 (C-7', C-11', C-15'), `-' 141.36 (C-4a), '-' 142.64 (C-5a oder C-9a), ’-' 144.14 (C-9a oder C-5a), ’-' 145.89 (C-10a), ’-' 160.74 (C-2). 
${ }^{1} \mathbf{H}$ NMR $\left(500 \mathrm{MHz}, \mathrm{CD}_{3} \mathrm{OD}\right): \delta=1.04\left(\mathrm{~d},{ }^{3} \mathrm{~J}_{3}{ }^{\prime} \mathrm{CH}_{3}, 3^{\prime} \mathrm{H}=6.6 \mathrm{~Hz}, 3 \mathrm{H}, 3^{\prime}-\mathrm{CH}_{3}\right), 1.27-1.37(\mathrm{~m}$, $\left.2 \mathrm{H}, 4^{\prime}-\mathrm{H}_{2}\right), 1.46-1.53\left(\mathrm{~m}, 1 \mathrm{H}, 3^{\prime}-\mathrm{H}\right), 1.54,1.55,1.56\left(3 \mathrm{~s}\right.$, je $3 \mathrm{H}, 11^{\prime}-\mathrm{CH}_{3}, 15^{\prime}-\mathrm{CH}_{3}, 1^{\prime}$ '$\left.\mathrm{CH}_{3}\right), 1.62\left(\mathrm{~s}, 3 \mathrm{H}, 7^{\prime}-\mathrm{CH}_{3}\right), 1.63\left(\mathrm{~d},{ }^{4} J_{20}{ }^{\prime} \mathrm{H}_{3}, 18^{\prime} \mathrm{H}=1.0 \mathrm{~Hz}, 3 \mathrm{H}, 20^{\prime}-\mathrm{H}_{3}\right), 1.70-1.79\left(\mathrm{~m}, 1 \mathrm{H}, 2^{\prime}-\right.$ H $\left.\mathrm{H}_{\mathrm{B}}\right) 1.79$ - $1.86\left(\mathrm{~m}, 1 \mathrm{H}, 2^{\prime}-\mathrm{H}_{\mathrm{A}}\right), 1.87$ - 2.17 (m, 14H, 5'- $\mathrm{H}_{2}, 8^{\prime} \mathrm{H}_{2}, 9^{\prime} \mathrm{H}_{2}, 12^{\prime}-\mathrm{H}_{2}, 13^{\prime}-\mathrm{H}_{2}, 16^{\prime}-$ $\left.\mathrm{H}_{2}, 17^{\prime}-\mathrm{H}_{2}\right), 4.29\left(\mathrm{~m}_{\mathrm{C}}, 2 \mathrm{H}, 1^{\prime}-\mathrm{H}_{2}\right), 4.99-5.06\left(\mathrm{~m}, 2 \mathrm{H}, 10^{\prime}-\mathrm{H}, 14^{\prime}-\mathrm{H}\right), 5.08\left(\mathrm{t} \mathrm{br},{ }^{3} J_{6} \mathrm{H}_{5} 5^{\prime} \mathrm{H}_{2}=\right.$ $7.9 \mathrm{~Hz}$, aufgespalten durch Fernkopplungen $\left.{ }^{4} J \approx 1.1 \mathrm{~Hz}, 1 \mathrm{H}, 6^{\prime}-\mathrm{H}\right), 5.15\left(\mathrm{t} \mathrm{br},{ }^{3} J_{18^{\prime} \mathrm{H}, 17^{\prime} \mathrm{H}_{2}}=7.0\right.$ $\mathrm{Hz}$, aufgespalten durch Fernkopplungen $\left.{ }^{4} J \approx 1.2 \mathrm{~Hz}, 1 \mathrm{H}, 18^{\prime}-\mathrm{H}\right), 7.42\left(\mathrm{~d},{ }^{4} J_{1 \mathrm{H}, 3 \mathrm{H}}=2.4 \mathrm{~Hz}\right.$, $1 \mathrm{H}, 1-\mathrm{H}), 7.58\left(\mathrm{dd},{ }^{3} J_{3 \mathrm{H}, 4 \mathrm{H}}=9.4 \mathrm{~Hz},{ }^{4} J_{4 \mathrm{H}, 1 \mathrm{H}}=2.5 \mathrm{~Hz}, 1 \mathrm{H}, 3-\mathrm{H}\right) 7.85\left(\mathrm{ddd},{ }^{3} J_{7 \mathrm{H}, 6 \mathrm{H}}=8.4 \mathrm{~Hz}\right.$, ${ }^{3} J_{7 \mathrm{H}, 8 \mathrm{H}}=6.7 \mathrm{~Hz},{ }^{4} J_{7 \mathrm{H}, 9 \mathrm{H}}=1.4 \mathrm{~Hz}, 1 \mathrm{H}, 7-\mathrm{H}$ oder $\left.8-\mathrm{H}\right), 7.91\left(\mathrm{ddd},{ }^{3} J_{8 \mathrm{H}, 9 \mathrm{H}}=8.5 \mathrm{~Hz},{ }^{4} J_{8 \mathrm{H}, 7 \mathrm{H}}=6.7\right.$ $\mathrm{Hz},{ }^{4} J_{8 \mathrm{H}, 6 \mathrm{H}}=1.5 \mathrm{~Hz}, 1 \mathrm{H}, 8-\mathrm{H}$ oder $\left.7-\mathrm{H}\right), 8.09\left(\mathrm{~d},{ }^{3} J_{4 \mathrm{H}, 3 \mathrm{H}}=9.4 \mathrm{~Hz}, 1 \mathrm{H}, 4-\mathrm{H}\right), 8.15\left(\mathrm{ddd},{ }^{3} J_{6 \mathrm{H}, 7 \mathrm{H}}\right.$ $=8.8 \mathrm{~Hz},{ }^{4} J_{6 \mathrm{H}, 8 \mathrm{H}}=0.8 \mathrm{~Hz},{ }^{5} J_{6 \mathrm{H}, 9 \mathrm{H}}=0.6 \mathrm{~Hz}, 1 \mathrm{H}, 6-\mathrm{H}$ oder $\left.9-\mathrm{H}\right), 8.19\left(\mathrm{ddd},{ }^{3} J_{9 \mathrm{H}, 7 \mathrm{H}}=8.5 \mathrm{~Hz}\right.$, ${ }^{4} J_{9 \mathrm{H}, 7 \mathrm{H}}=1.4 \mathrm{~Hz},{ }^{5} J_{9 \mathrm{H}, 6 \mathrm{H}}=0.6 \mathrm{~Hz}, 1 \mathrm{H}, 9-\mathrm{H}$ oder $\left.6-\mathrm{H}\right)$.

MS $(70 \mathrm{eV}): m / z(\%)=538(100)\left[\mathrm{M}^{+}\right], 470(6)\left[\mathrm{M}^{+}-\mathrm{C}_{5} \mathrm{H}_{8}\right], 402(8)\left[470-\mathrm{C}_{5} \mathrm{H}_{8}\right], 334$ (20) [402- $\left.\mathrm{C}_{5} \mathrm{H}_{8}\right], 265$ (4) [334- $\left.\mathrm{C}_{5} \mathrm{H}_{9}\right], 196(82)\left[\mathrm{C}_{12} \mathrm{H}_{8} \mathrm{~N}_{2} \mathrm{O}^{+}\right], 168$ (7) [196-CO].

HR-MS: ber. für $\mathrm{C}_{37} \mathrm{H}_{50} \mathrm{~N}_{2} \mathrm{O}: 538.3923$; gef.: 538.3923 . 


\section{Synthese von $(S)$-Methanophenazin $[(S)-1]$}

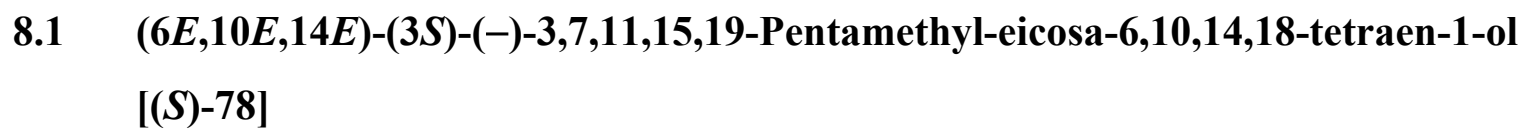

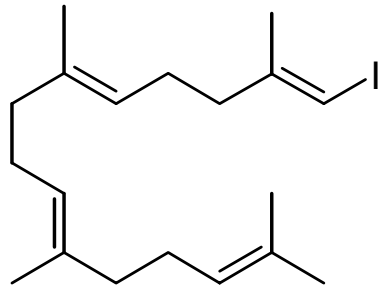

7
1. (R)-6, $\mathrm{ZnCl}_{2}, \mathrm{Et}_{2} \mathrm{O}, \mathrm{RT}$, dann tert.-BuLi, $-90^{\circ} \mathrm{C} \longrightarrow \mathrm{RT}$, Transfer zu 7, kat. $\mathrm{Pd}\left(\mathrm{PPh}_{3}\right)_{4}, \mathrm{RT}$

2. TBAF,THF, $67^{\circ} \mathrm{C}$

2 Stufen $64 \%$

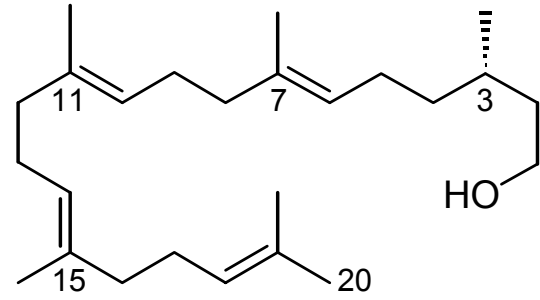

$(S)-78$

Es wurden $102 \mathrm{mg}$ (0.78 mmol, 1.5 Äquiv.) Zinkchlorid i. Vak. mit einer Heißluftpistole geschmolzen und in einer Argon-Atmosphäre auf Raumtemperatur abgekühlt. Anschließend versetzt man das trockene Zinkchlorid mit einer Lösung aus $268 \mathrm{mg}$ (0.78 mmol, mmol, 1.5 Äquiv.) des chiralen Alkyliodids (R)-6 in $10 \mathrm{ml}$ Dietylether und rührte bei Raumtemperatur etwa $1 \mathrm{~h}$, bis sich das Zinkchlorid vollständig gelöst hatte. Eine Kältemischung, bestehend aus Petrolether/Isopropanol/Aceton im Verhältnis $4: 1: 1$, wurde mit flüssigem Stickstoff auf $-100{ }^{\circ} \mathrm{C}$ gebracht und die Reaktionsmischung darin abgekühlt. Bei dieser Temperatur tropfte man $1.5 \mathrm{ml}$ (2.25 mmol, 4.5 Äquiv.) einer 1.5 M Lösung von tert.-Butyllithium in Pentan mit einer Spritze langsam zu, rührte noch $1 \mathrm{~h}$ in der Kälte, entfernte dann das Kältebad und ließ die Reaktionsmischung auftauen. Nach 1 h Rühren bei Raumtemperatur wurde der Ansatz unter Ausschluß von Luftfeuchtigkeit durch eine an beiden Seiten spitze Kanüle unter einem leichten Überdruck von Argon in einen separaten Kolben übergeführt, der 193 mg (0.5 mmol, 1.0 Äquiv.) Vinyliodid 7 und $30 \mathrm{mg}$ (0.043 mmol, 0.09 Äquiv.) Tetrakistriphenylphosphinpalladium enthielt. Nach 6 h Rühren bei Raumtemperatur wurde die Reaktionsmischung zur Aufarbeitung in $20 \mathrm{ml}$ Diethylether aufgenommen, zweimal mit je $10 \mathrm{ml}$ gesättigter Natriumchloridlösung gewaschen, über Natriumsulfat getrocknet, filtriert und das Solvens am Rotationsverdampfer i. Vak. entfernt. Das Rohprodukt wurde über 5 g Kieselgel $\left(\mathrm{PE} / \mathrm{Et}_{2} \mathrm{O}=\right.$ 50 : 1) filtriert und dann in $4.5 \mathrm{ml}$ ( $1.5 \mathrm{mmol}$ Fluorid, 3.0 Äquiv.) einer $0.33 M$ Lösung aus Tetrabutylammoniumfluorid-Trihydrat in Tetrahydrofuran aufgenommen. Nach $1 \mathrm{~h}$ Erhitzen auf $70{ }^{\circ} \mathrm{C}$ Ölbadtemperatur (DC Kontrolle: $\mathrm{Et}_{2} \mathrm{O} / \mathrm{PE}=1: 1$ ) ließ man den Ansatz auf Raumtemperatur abkühlen und entfernte das Solvens i. Vak. am Rotationsverdampfer. Den Rückstand zog man auf ca. $500 \mathrm{mg}$ Kieselgel auf und chromatographierte an 6 g Kieselgel 
$\left(\mathrm{Et}_{2} \mathrm{O} / \mathrm{PE}=1: 50 \rightarrow 1: 1\right)$. Es wurden $115 \mathrm{mg}$ (64\% Ausbeute bezogen auf das Vinyliodid 7) der Titelverbindung $(S)-\mathbf{7 8}$ als klares Öl isoliert.

$\mathbf{R}_{\mathbf{f}}=0.47\left(\mathrm{Et}_{2} \mathrm{O} / \mathrm{PE}=1: 1\right)$.

$[\alpha]_{D}^{20}=-2.6^{\circ}\left(c=1\right.$ in $\left.\mathrm{CHCl}_{3}\right)$.

${ }^{1} \mathbf{H}$ NMR $\left(300 \mathrm{MHz}, \mathrm{C}_{6} \mathrm{D}_{6}\right): \delta=0.90\left(\mathrm{~d},{ }^{3} \mathrm{~J}_{3 \mathrm{CH}_{3}, 3 \mathrm{H}}=6.0 \mathrm{~Hz}, 3 \mathrm{H}, 3-\mathrm{CH}_{3}\right), 1.10-1.51(\mathrm{~m}, 5 \mathrm{H}$, 2- $\left.\mathrm{H}_{2}, 4-\mathrm{H}_{2}, \mathrm{OH}\right), 1.53-1.85(\mathrm{~m}, 1 \mathrm{H}, 3-\mathrm{H})$ überlagert von $1.66\left(\mathrm{~s}, 3 \mathrm{H}, 19-\mathrm{CH}_{3}\right), 1.71(\mathrm{~s}, 9 \mathrm{H}, 7-$ $\left.\mathrm{CH}_{3}, 11-\mathrm{CH}_{3}, 15-\mathrm{CH}_{3}\right), 1.78\left(\mathrm{~s}, 3 \mathrm{H}, 20-\mathrm{H}_{3}\right), 2.05-2.43\left(\mathrm{~m}, 14 \mathrm{H}, 5-\mathrm{H}_{2}, 8-\mathrm{H}_{2}, 9-\mathrm{H}_{2}, 12-\mathrm{H}_{2}, 13-\right.$ $\left.\mathrm{H}_{2}, 16-\mathrm{H}_{2}, 17-\mathrm{H}_{2}\right), 3.55\left(\mathrm{~m}_{\mathrm{C}}, 2 \mathrm{H}, 1-\mathrm{H}_{2}\right), 5.21$ - 5.50 (m, 4H, 6-H, 10-H, 14-H, 18-H).

${ }^{13} \mathrm{C}$ NMR (50 MHz, $\left.\mathrm{C}_{6} \mathrm{D}_{6}\right): \delta={ }^{\prime}+16.09$, ' +' 16.12, '+' $16.15\left(7-\mathrm{CH}_{3}, 11-\mathrm{CH}_{3}, 15-\mathrm{CH}_{3}\right)$, ' +' $17.75\left(19-\mathrm{CH}_{3}\right),{ }^{\prime}+19.71\left(3-\mathrm{CH}_{3}\right)$, '-' $25.84(\mathrm{C}-5)$, '+' $25.86(\mathrm{C}-20)$, '-' $25.88(\mathrm{C}-2)$, '-' 27.11 und '-' 27.23 (C-9, C-13, C-17), '+' 29.49 (C-3), '-' 37.64 (C-4), '-' 40.21 (C-8, C-12, $\mathrm{C}-16)$, '-' $60.83(\mathrm{C}-1)$, ' +'124.80 (C-10 und C-14), '+' 124.93 (C-6), '+' 125.27 (C-18), '-' 131.10 (C-19), '-' 134.78, '-' 134.96, '-' 134.99 (C-7, C-11, C-15).

\section{$\mathrm{C}_{25} \mathrm{H}_{44} \mathrm{O}(360.34)$}

\section{2 (E6',E10',E14')-(3'S)-(-)-2-(3,7,11,15,19-Pentamethyl-eicosa-6,10,14,18-} tetraenyloxy)-phenazin $[(S)-1]$<smiles>CC(C)=CCCC(C)=CCCC(C)=CCC[C@@H](C)CCO</smiles>

1. $\mathrm{THF}, \mathrm{NEt}_{3}, \mathrm{CH}_{3} \mathrm{SO}_{2} \mathrm{Cl}, 0^{\circ} \mathrm{C} \rightarrow \mathrm{RT}$, 2. 2-Hydroxyphenazin (2), $\mathrm{KOH}$, Aliquat 336, Toluol, $110^{\circ} \mathrm{C}$ $90 \%$<smiles>CC(C)=CCC/C(C)=C/CC/C(C)=C/CC/C(C)=C/CC[C@H](C)CCOc1ccc2nc3ccccc3nc2c1</smiles>

(S)-1

Man löste in $2 \mathrm{ml}$ trockenem Tetrahydrofuran (K) $54 \mathrm{mg}$ (0.15 mmol, 1.0 Äquiv.) des enantiomerenreinen Alkohols $(S)-78$ und brachte ihn bei $0{ }^{\circ} \mathrm{C}$ mit $30 \mu \mathrm{l}(21.8 \mathrm{mg}, 0.20 \mathrm{mmol}$, 1.3 Äquiv.) frisch destilliertem Triethylamin und $19 \mathrm{mg}$ (0.165 mmol, 1.1 Äquiv.) Methansulfonsäurechlorid zur Reaktion. Nach etwa 15 min war der Alkohol $(S)$-78 quantitativ umgesetzt (DC Kontrolle: $\mathrm{Et}_{2} \mathrm{O} / \mathrm{PE}=1: 3$ ). Der Ansatz wurde in $5 \mathrm{ml}$ gesättigte Natriumhydrogencarbonatlösung gegeben und viermal mit je $5 \mathrm{ml}$ Diethylether ausgeschüttelt, über 
Natriumsulfat getrocknet, filtriert und das Lösungsmittel am Rotationsverdampfer i. Vak. evaporiert. In einem separaten Reaktionskolben suspendierte man 35 mg (0.18 mmol, 1.2 Äquiv.) 2-Hydroxyphenazin (2), $10 \mathrm{mg}$ wasserfreies, fein gemörsertes Kaliumhydroxid und ca. $20 \mathrm{mg}$ [ca. 30 Gew.\% bezogen auf den Alkohol (S)-78] Aliquat 336 in $5 \mathrm{ml}$ Toluol und rührte $30 \mathrm{~min}$ bei Raumtemperatur. $\mathrm{Zu}$ dieser roten Suspension fügte man dann das rohe Mesylat als Lösung in $2 \mathrm{ml}$ Toluol und erhitzte unter kräftigem Rühren für $3 \mathrm{~h}$ unter Sieden am Rückfluß (DC Kontrolle: $\mathrm{PE} / \mathrm{CH}_{2} \mathrm{Cl}_{2}=1: 100$ ). Nach dem Abkühlen wurde der Ansatz in $5 \mathrm{ml}$ Wasser aufgenommen und viermal mit je $5 \mathrm{ml}$ Diethylether extrahiert. Die vereinigten organischen Phasen wurden getrocknet, filtriert und das Solvens i. Vak. entfernt. Das verbliebene Rohprodukt wurde auf $1 \mathrm{~g}$ Kieselgel aufgezogen und an $35 \mathrm{~g}$ Kieselgel chromatographiert (Eluent: $\mathrm{PE} / \mathrm{CH}_{2} \mathrm{Cl}_{2}=1$ : 100). Es wurden $72.5 \mathrm{mg}$ (90\% Ausbeute) der Titelverbindung $(S)-\mathbf{1}$ als gelbes Ö1 isoliert.

$\mathbf{R}_{\mathbf{f}}=0.27\left(\mathrm{PE} / \mathrm{CH}_{2} \mathrm{Cl}_{2}=1: 100\right)$.

$\mathbf{U V}\left(\mathrm{CH}_{3} \mathrm{CN}\right): \lambda_{\max }(\lg \varepsilon)=256 \mathrm{~nm}$ (4.89), 354 (3.95), 388 (3.89).

$[\alpha]_{D}^{20}=-3.7^{\circ}\left(c=1\right.$ in $\left.\mathrm{C}_{6} \mathrm{H}_{6}\right) ;[\alpha]_{\mathbf{5 7 8}}^{20}=-4.0^{\circ}\left(c=1\right.$ in $\left.\mathrm{C}_{6} \mathrm{H}_{6}\right) ;[\alpha]_{546}^{20}=-4.6^{\circ}\left(c=1\right.$ in $\left.\mathrm{C}_{6} \mathrm{H}_{6}\right)$.

${ }^{1} \mathbf{H}$ NMR $\left(200 \mathrm{MHz}, \mathrm{C}_{6} \mathrm{D}_{6}\right): \delta=0.89\left(\mathrm{~d},{ }^{3} J_{3}{ }^{\prime} \mathrm{CH}_{3}, \mathrm{CH}=6.5 \mathrm{~Hz}, 3 \mathrm{H}, 3^{\prime}-\mathrm{CH}_{3}\right), 1.12-1.58(\mathrm{~m}, 4 \mathrm{H}$, 4'- $\left.\mathrm{H}_{2}, 2^{\prime}-\mathrm{H}_{2}\right), 1.61$ (s, 3H, 19'- $\left.\mathrm{H}_{3}\right), 1.70-1.80$ (m, 1H, 3'-H) überlagert von 1.64 - 1.71 (3 s, je $\left.3 \mathrm{H}, 7^{\prime}-\mathrm{CH}_{3}, 11^{\prime}-\mathrm{CH}_{3}, 15^{\prime}-\mathrm{CH}_{3}\right)$ und $1.73\left(\mathrm{~s}, 3 \mathrm{H}, 20^{\prime}-\mathrm{H}_{3}\right), 2.02-2.29$ (m, $14 \mathrm{H}, 5^{\prime}-\mathrm{H}_{2}, 8^{\prime}-\mathrm{H}_{2}$, $\left.9^{\prime}-\mathrm{H}_{2}, 12^{\prime}-\mathrm{H}_{2}, 13^{\prime}-\mathrm{H}_{2}, 16^{\prime}-\mathrm{H}_{2}, 17^{\prime}-\mathrm{H}_{2}\right), 3.83\left(\mathrm{t},{ }^{3} J_{1}{ }^{\prime} \mathrm{H}_{2}, 2^{\prime} \mathrm{H}_{2}=6.5,2 \mathrm{H}, 1^{\prime}-\mathrm{H}_{2}\right), 5.21-5.48(\mathrm{~m}$, $4 \mathrm{H}, 6$ '- $\left.\mathrm{H}, 10^{\prime}-\mathrm{H}, 14^{\prime}-\mathrm{H}, 18^{\prime}-\mathrm{H}\right), 7.27-7.47$ (m, 3H, 3-H, 7-H, 8-H), $7.56\left(\mathrm{~d},{ }^{4} J_{1 \mathrm{H}, 3 \mathrm{H}}=3.0 \mathrm{~Hz}\right.$, $1 \mathrm{H}, 1-\mathrm{H}), 8.15\left(\mathrm{~d},{ }^{3} J_{4 \mathrm{H}, 3 \mathrm{H}}=9.6 \mathrm{~Hz}, 1 \mathrm{H}, 4-\mathrm{H}\right), 8.34\left(\mathrm{dd},{ }^{3} J_{6 \mathrm{H}, 7 \mathrm{H}}=7.0 \mathrm{~Hz},{ }^{4} J_{6 \mathrm{H}, 8 \mathrm{H}}=2.5 \mathrm{~Hz}, 1 \mathrm{H}\right.$, $6-\mathrm{H}$ und dd, $\left.{ }^{3} J_{9 \mathrm{H}, 8 \mathrm{H}}=7.0 \mathrm{~Hz},{ }^{4} J_{9 \mathrm{H}, 7 \mathrm{H}}=2.5 \mathrm{~Hz}, 1 \mathrm{H}, 9-\mathrm{H}\right)$.

${ }^{13} \mathbf{C}$ NMR (50 MHz, $\left.\mathrm{C}_{6} \mathrm{D}_{6}\right): \delta={ }^{+}{ }^{\prime} 16.12\left(7^{\prime}-\mathrm{CH}_{3}, 11^{\prime}-\mathrm{CH}_{3}, 15^{\prime}-\mathrm{CH}_{3}\right),{ }^{\prime}+17.73\left(19^{\prime}-\mathrm{CH}_{3}\right)$, '+' $19.57\left(3^{\prime}-\mathrm{CH}_{3}\right)$, '+' $25.76(\mathrm{C}-20$ '), '-' 25.85 (C-5'), '-' 27.10 und '-' 27.20 (C-9', C-13', C-17'), '+' 29.76 (C-3'), '-' 35.99 (C-2'), '-' 37.43 (C-4'), '-' 40.19 und '-' 40.22 (C-8', C12', C-16'), ’-' $66.86(\mathrm{C}-1$ '), ’+' 105.78 (C-1), '+' 124.72, '+' 124.75, ’+' 124.91, (C-10', C14 ', C-18'), '+' $125.02\left(\mathrm{C}-6\right.$ '), ’+' $126.51(\mathrm{C}-3),{ }^{\prime}+128.76(\mathrm{C}-7$ oder C-8), ’+' $129.58(\mathrm{C}-8$ oder C-7), '+' 130.14 (C-6 oder C-9), '+' 130.27 (C-9 oder C-6), '-' 131.07 (C-19'), '+' 131.28 (C-4), '-' 134.98, '-' 135.01, '-' 135.07 (C-7', C-11', C-15'), ’-' 141.34 (C-4a), '-' 142.61 (C-5a oder C-9a), '-' 144.11 (C-9a oder C-5a), '-' 145.87 (C-10a), '-' 160.73 (C-2).

\section{$\mathrm{C}_{37} \mathrm{H}_{50} \mathrm{~N}_{2} \mathrm{O}$ (538.39)}




\section{Synthese der Mosher-Ester rac-63, $(S, S)-63$, rac-79 und $(S, S)-79$}

\section{$9.1 \quad\left(2 S, 3^{\prime} R S\right)-3,3,3$-Trifluoro-2-methoxy-2-phenyl-propansäure-5'-(tert.-butyl- dimethyl-silanyloxy)-3-methyl-pentylester (rac-63)}

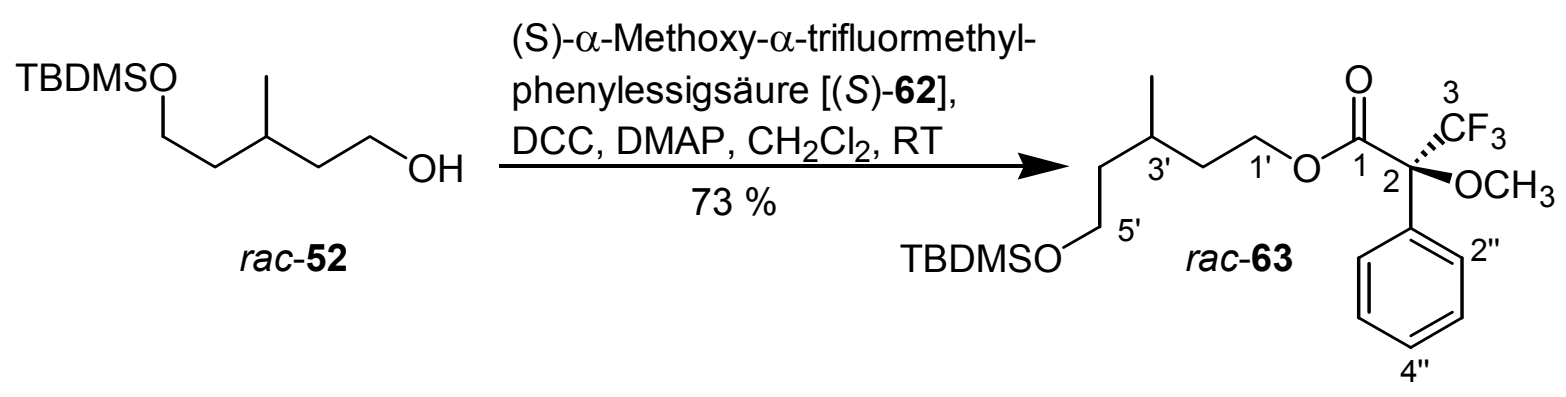

Es wurden $13 \mathrm{mg}$ (0.055 mmol, 1.1 Äquiv.) der enantiomerenreinen $(S)$-(-)- $\alpha$-Methoxy- $\alpha-$ trifluormethylphenylessigsäure $[(S)-62]$ in $0.5 \mathrm{ml}$ trockenem Dichlormethan gelöst, mit $11.3 \mathrm{mg}$ (0.055 mmol, 1.1 Äquiv.) Dicyclohexylcarbodiimid, $0.8 \mathrm{mg}$ (0.007 mmol, 0.013 Äquiv.) 4-Dimethylaminopyridin versetzt und bei Raumtemperatur gerührt. Dann spritzte man $11.5 \mathrm{mg}$ (0.05 mmol, 1.0 Äquiv.) des racemischen Alkohols rac-52 zu und rührte weitere 12 h. Zur Aufarbeitung gab man 200 mg Kieselgel zur Reaktionsmischung und evaporierte das Solvens am Rotationsverdampfer i. Vak. Das so auf Kieselgel aufgezogene Rohprodukt wurde an $5 \mathrm{~g}$ Kieselgel chromatographisch gereinigt $\left(\mathrm{Et}_{2} \mathrm{O} / \mathrm{PE}=1: 10\right)$. Es wurden $16.2 \mathrm{mg}$ (73\% Ausbeute) der Titelverbindung rac-63 als ein Gemisch von zwei Diastereomeren im Verhältnis $1: 1$ isoliert.

$\mathbf{R}_{\mathbf{f}}=0.68\left(\mathrm{Et}_{2} \mathrm{O} / \mathrm{PE}=1: 2\right)$.

${ }^{13} \mathrm{C}$ NMR (50 MHz, $\mathrm{C}_{6} \mathrm{D}_{6}$, Diastereomerengemisch): $\delta={ }^{+}{ }^{\prime}-5.31,{ }^{\prime}+{ }^{\prime}-5.88\left[\mathrm{Si}\left(\mathrm{CH}_{3}\right)_{2}\right]$, '-'

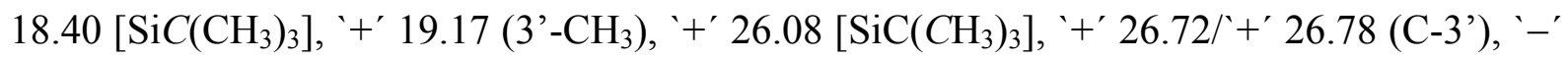

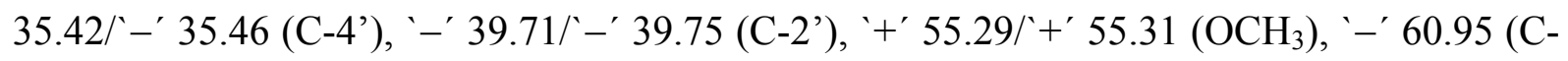
5'), '-' 64.64/'-' $64.70\left(\mathrm{C}-1\right.$ '), ' -' $85.11\left(\mathrm{~d},{ }^{2} J_{\mathrm{C} 1, \mathrm{~F}_{3}}=28 \mathrm{~Hz}, \mathrm{C}-2\right),{ }^{\prime}-{ }^{\prime} 126.30\left(\mathrm{q},{ }^{1} J_{\mathrm{CF}_{3}}=288\right.$ $\mathrm{Hz}, \mathrm{CF}_{3}$ ), '+' 127.52 (C-3', und C-5'’), '+' 128.57 (C-2', und C-6'”), '+' 129.65 (C-4' '), '-' 133.14 (C-1"').

${ }^{1} \mathbf{H}$ NMR $\left(500 \mathrm{MHz}, \mathrm{C}_{6} \mathrm{D}_{6}\right): \delta=0.05\left[\mathrm{~s}, 12 \mathrm{H}, \mathrm{Si}\left(\mathrm{CH}_{3}\right)_{2}\right], 0.705\left(\mathrm{~d},{ }^{3} J_{3^{\prime}} \mathrm{CH}_{3}, 3^{\prime} \mathrm{H}=7.0 \mathrm{~Hz}, 3 \mathrm{H}\right.$, $3^{\prime}-\mathrm{CH}_{3}$, Diastereomer A) teilweise überlagert von $0.710\left(\mathrm{~d}^{3}, J_{3}{ }^{\prime} \mathrm{CH}_{3}, 3^{\prime} \mathrm{H}=7.0 \mathrm{~Hz}, 3 \mathrm{H}, 3^{\prime}-\mathrm{CH}_{3}\right.$, Diastereomer B), 0.98 [s, 9H, $\mathrm{SiC}\left(\mathrm{CH}_{3}\right)_{3}$, Diastereomer A] teilweise überlagert von 0.985 [s, 9H, $\mathrm{SiC}\left(\mathrm{CH}_{3}\right)_{3}$, Diastereomer B], 1.13 - 1.28 (m, 4H, 4'- $\left.\mathrm{H}_{2}\right), 1.35$ - 1.63 (m, 6H, 2'- $\mathrm{H}_{2}, 3^{\prime}$ '- 
$\mathrm{H}), 3.43$ - $3.53\left(\mathrm{~m}, 4 \mathrm{H}, 5^{\prime}-\mathrm{H}_{2}\right)$ überlagert von $3.45\left(\mathrm{~d},{ }^{5} \mathrm{~J}_{\mathrm{CF}_{3}, \mathrm{OCH}_{3}}=1.3 \mathrm{~Hz}, 6 \mathrm{H}, \mathrm{OCH}_{3}\right), 4.07$ -

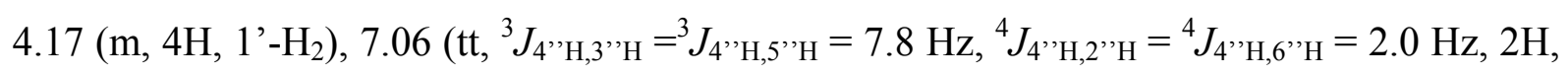

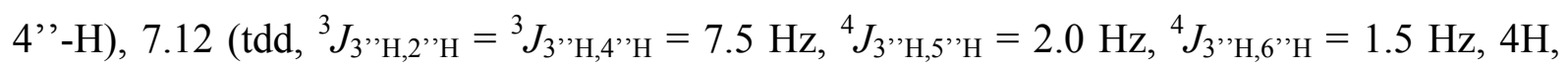

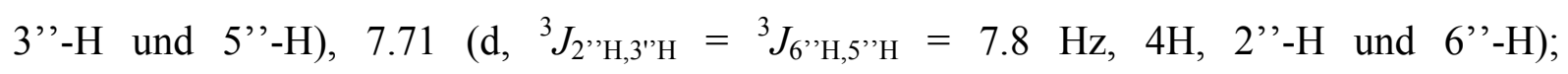
Diastereomerenverhältnis = $1: 1$ (bestimmt durch Vergleich der Signalintensitäten von 3'$\mathrm{CH}_{3}$ bei $\left.\delta=0.705 / 0.710 \mathrm{ppm}\right)$.

${ }^{19}$ F NMR $\left(470 \mathrm{MHz}, \mathrm{C}_{6} \mathrm{D}_{6}\right): \delta=-72.144\left(\mathrm{CF}_{3}\right.$, Diastereomer A) überlagert von -72.156 $\left(\mathrm{CF}_{3}\right.$, Diastereomer B); Diastereomerenverhältnis $=1: 1$

\section{$\mathrm{C}_{22} \mathrm{H}_{35} \mathrm{~F}_{3} \mathrm{SiO}_{4}$ (448.60)}

\section{2 (2S,3'S)-3,3,3-Trifluoro-2-methoxy-2-phenyl-propansäure-5'-(tert.- butyldimethyl-silanyloxy)-3-methyl-pentylester [(S)-63]}

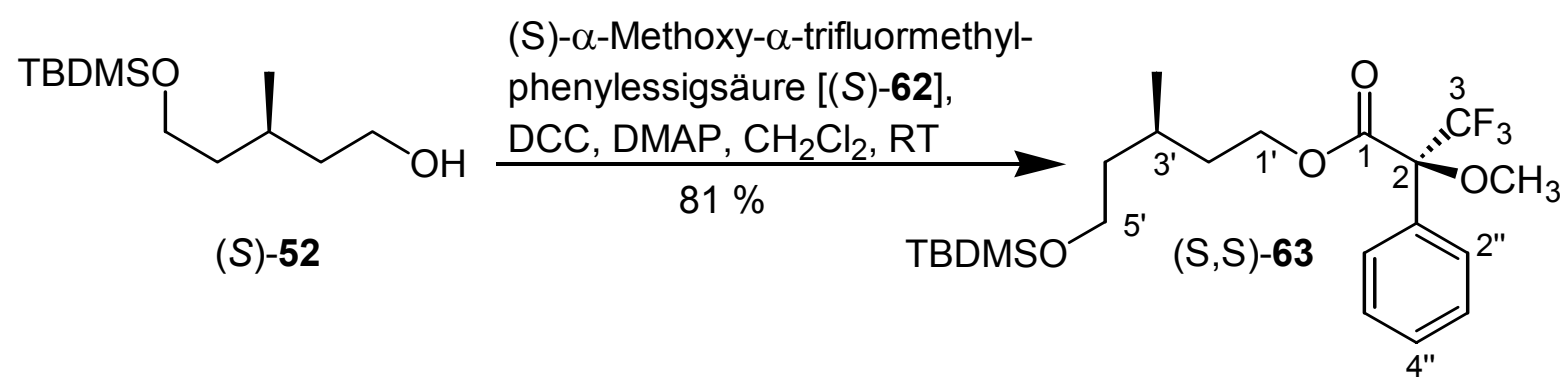

Es wurden $10 \mathrm{mg}(0.042 \mathrm{mmol}, 1.1$ Äquiv.) der enantiomerenreinen $(S)-(-)-\alpha$-Methoxy- $\alpha$ trifluormethylphenylessigsäure $[(S)-62]$ in $0.5 \mathrm{ml}$ trockenem Dichlormethan gelöst, mit $8.8 \mathrm{mg}$ (0.042 mmol, 1.1 Äquiv.) Dicyclohexylcarbodiimid, $0.5 \mathrm{mg}$ (0.004 mmol, 0.01 Äquiv.) 4-Dimethylaminopyridin versetzt und bei Raumtemperatur gerührt. Dann spritzte man $8.9 \mathrm{mg}$ (0.038 mmol, 1.0 Äquiv.) des enantiomerenreinen Alkohols $(S)$-52 zu und rührte weitere 12 h. Zur Aufarbeitung gab man 200 mg Kieselgel zur Reaktionsmischung und evaporierte das Solvens am Rotationsverdampfer i. Vak. Das so auf Kieselgel aufgezogene Rohprodukt wurde an $5 \mathrm{~g}$ Kieselgel chromatographiert $\left(\mathrm{Et}_{2} \mathrm{O} / \mathrm{PE}=1: 10\right)$. Es wurden 13.9 mg des diastereomerenreinen Mosher-Esters $(S, S)$-63 (81\% Ausbeute) isoliert. 
$\mathbf{R}_{\mathbf{f}}=0.68\left(\mathrm{Et}_{2} \mathrm{O} / \mathrm{PE}=1: 2\right)$.

${ }^{1} \mathbf{H}$ NMR $\left(500 \mathrm{MHz}, \mathrm{C}_{6} \mathrm{D}_{6}\right.$, Diastereomer A): $\delta=0.09\left[\mathrm{~s}, 6 \mathrm{H}, \mathrm{Si}\left(\mathrm{CH}_{3}\right)_{2}\right], 0.745\left(\mathrm{~d},{ }^{3} J_{3^{\prime} \mathrm{CH}_{3}, 3^{3} \mathrm{H}}\right.$ $\left.=7.0 \mathrm{~Hz}, 3 \mathrm{H}, 3^{\prime}-\mathrm{CH}_{3}\right), 1.02\left[\mathrm{~s}, 9 \mathrm{H}, \mathrm{SiC}\left(\mathrm{CH}_{3}\right)_{3}\right], 1.17-1.31\left(\mathrm{~m}, 2 \mathrm{H}, 4^{\prime}-\mathrm{H}_{2}\right), 1.31$ - $1.66(\mathrm{~m}$, $\left.3 \mathrm{H}, 4-\mathrm{H}_{2}, 3^{\prime}-\mathrm{H}\right), 3.47-3.59\left(\mathrm{~m}, 2 \mathrm{H}, 5^{\prime}-\mathrm{H}_{2}\right)$ überlagert von $3.49\left(\mathrm{~d},{ }^{5} J_{\mathrm{CF}_{3}, \mathrm{OCH}_{3}}=1.3 \mathrm{~Hz}, 3 \mathrm{H}\right.$,

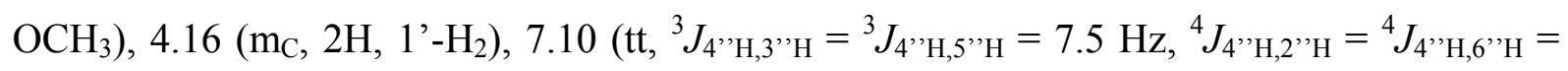

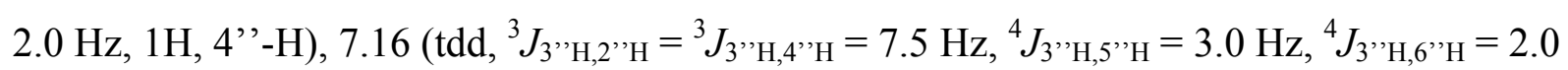

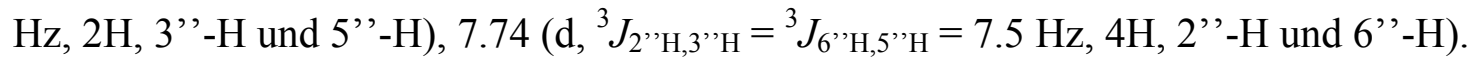

$\mathrm{C}_{22} \mathrm{H}_{35} \mathrm{~F}_{3} \mathrm{SiO}_{4}$ (448.60)

9.3 (2S,3' $\left.R S, 6^{\prime} E, 1^{\prime}{ }^{\prime} E, 14^{\prime} E, 18^{\prime} E\right)-3,3,3$-Trifluoro-2-methoxy-2-phenyl-propansäure3',7',11',15',19'-pentamethyl-eicosa-6',10',14',18'-tetraenyl-ester (rac-79)

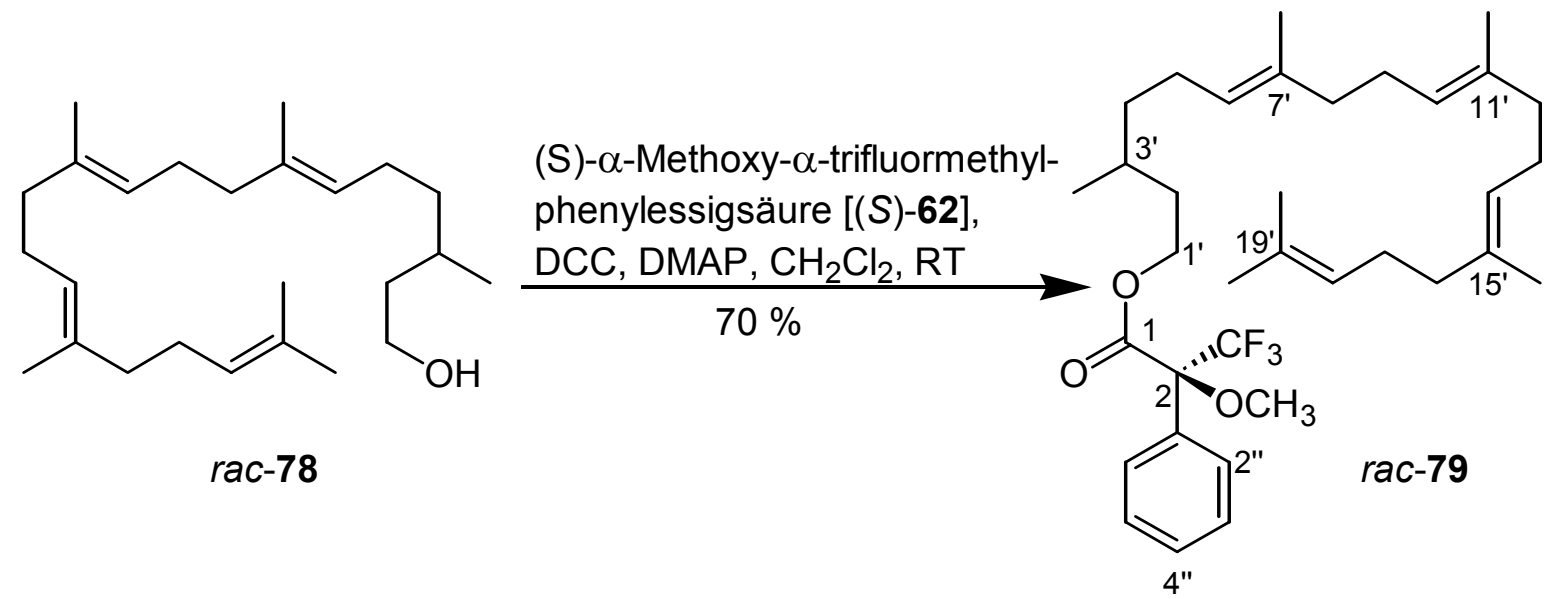

Es wurden $7.7 \mathrm{mg}$ (0.033 mmol 1.1 Äquiv.) der enantiomerenreinen $(S)-(-)-\alpha-$ Methoxy- $\alpha$ trifluormethylphenylessigsäure [(S)-62] in $0.5 \mathrm{ml}$ trockenem Dichlormethan gelöst und 6.8 mg (0.033 mmol, 1.1 Äquiv.) Dicyclohexylcarbodiimid sowie $0.5 \mathrm{mg}$ (0.004 mmol, 0.12 Äquiv.) 4-Dimethylaminopyridin zugegeben. Nach $10 \mathrm{~min}$ versetzte man mit $10.8 \mathrm{mg}(0.03$ mmol, 1.0 Äquiv.) des racemischen Alkohols rac-78 und rührte $10 \mathrm{~h}$ bei Raumtemperatur. Abschließend fügte man $200 \mathrm{mg}$ Kieselgel zur Reaktionsmischung, entfernte alles Flüchtige i. Vak. am Rotationsverdampfer und reinigte den Rückstand an 2 g Kieselgel $\left(\mathrm{Et}_{2} \mathrm{O} / \mathrm{PE}=\right.$ $1:$ 100). Es wurden $11.8 \mathrm{mg}$ (70\% Ausbeute) der Titelverbindung rac-79 als Gemisch von zwei Diastereomeren im Verhältnis $1: 1$ isoliert. 
$\mathbf{R}_{\mathbf{f}}=0.76\left(\mathrm{Et}_{2} \mathrm{O} / \mathrm{PE}=1: 3\right)$.

${ }^{13} \mathrm{C}$ NMR (125 MHz, $\left.\mathrm{C}_{6} \mathrm{D}_{6}\right): \delta={ }^{+}+16.04,{ }^{\prime}+' 16.10,{ }^{\prime}+{ }^{\prime} 16.12\left(7^{\prime}-\mathrm{CH}_{3}, 11^{\prime}-\mathrm{CH}_{3}, 15^{\prime}-\mathrm{CH}_{3}\right)$,

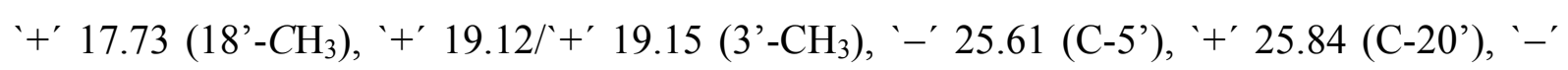
27.10, '-' 27.11, '-' 27.22 (C-9', C-13', C-17'), '-' 35.35/ -' 35.38 (C-4'), '-' 37.05/`-' 37.08 (C-2'), '-' 40.20 (C-8', C-12', C-16'), '+' $55.29\left(\mathrm{OCH}_{3}\right)$, '-' 64.69/`-' $64.72(\mathrm{C}-1$ ' $)$, '-' $88.11\left(\mathrm{~d},{ }^{2} J_{\mathrm{C}_{1, \mathrm{~F}_{3}}}=28 \mathrm{~Hz}, \mathrm{C}-2\right)$, '-' $124.26\left(\mathrm{q},{ }^{1} J_{\mathrm{CF}_{3}}=288 \mathrm{~Hz}, \mathrm{CF}_{3}\right)$, '+' 124.74, '+' 124.77, '+' $124.80 /{ }^{\prime}+124.82$, ' $^{\prime} 124.92$ (C-6', C-10', C-16, C-18'), '+' 127.77 (C-3’’ und C-5',), '+' 128.58 (C-2', und C-6', '), '+' 129.67 (C-4' '), '-' 131.11 (C-19'), '-' 133.16 (C1'’), '-' 135.01, '-' 135. 06, '-' 135.09 (C-7', C-11', C-15'), '-' 166.60 (C=O); Diastereomerenverhältnis $=1: 1$ (bestimmt durch Vergleich der Signalintensitäten von C-1' bei $\delta=$ $64.69 / 64.72$ ppm, C-2' bei $\delta=37.05 / 37.08$ ppm, C-4' bei $\delta=35.35 / 35.38$ ppm sowie 3'- $\mathrm{CH}_{3}$ bei $\delta=19.12 / 19.15 \mathrm{ppm})$.

${ }^{1} \mathbf{H}$ NMR (300 MHz, $\mathrm{C}_{6} \mathrm{D}_{6}$, Diastereomerengemisch): $\delta=0.79\left(\mathrm{~d},{ }^{3} J_{3}{ }^{\prime} \mathrm{CH}_{3}, 3^{\prime} \mathrm{H}=6.5 \mathrm{~Hz}, 6 \mathrm{H}, 3\right.$ ' $\left.\mathrm{CH}_{3}\right), 1.06$ - 1.39 (m, 8 H, 2'- $\left.\mathrm{H}_{2}, 4^{\prime}-\mathrm{H}_{2}\right), 1.39$ - 1.65 (m, 2H, 3'-H), 1.67 (s, 6H, 19'-CH CH$_{3}$, überlagert von $1.68\left(\mathrm{~s}, 6 \mathrm{H}, 7^{\prime}-\mathrm{CH}_{3}\right), 1.72\left(2 \mathrm{~s}\right.$ br, je $6 \mathrm{H}, 11^{\prime}-\mathrm{CH}_{3}$ und 15'- $\left.\mathrm{CH}_{3}\right)$, $1.78\left(\mathrm{~d},{ }^{4} \mathrm{~J}_{20}{ }^{\prime} \mathrm{H}, 18^{\prime} \mathrm{H}=1.0 \mathrm{~Hz}, 6 \mathrm{H}, 20^{\prime}-\mathrm{H}_{3}\right), 2.02\left(\mathrm{~m}_{\mathrm{C}}, 4 \mathrm{H}, 5^{\prime}-\mathrm{H}_{2}\right), 2.15-2.38\left(\mathrm{~m}, 24 \mathrm{H}, 8^{\prime}-\mathrm{H}_{2}\right.$, $\left.9^{\prime}-\mathrm{H}_{2}, 12^{\prime}-\mathrm{H}_{2}, 14^{\prime}-\mathrm{H}_{2}, 16^{\prime}-\mathrm{H}_{2}, 17^{\prime}-\mathrm{H}_{2}\right), 3.53\left(\mathrm{~d},{ }^{5} J_{\mathrm{CF}_{3}, \mathrm{OCH}_{3}}=1.5 \mathrm{~Hz}, 3 \mathrm{H}, \mathrm{OCH}_{3}\right), 4.11-4.29$ (m, 4H, 1'- $\left.\mathrm{H}_{2}\right), 5.28\left(\mathrm{t} \mathrm{br},{ }^{3} J_{18^{\prime} \mathrm{H}, 17^{\prime} \mathrm{H}}=7.0 \mathrm{~Hz}, 2 \mathrm{H}, 20-\mathrm{H}\right), 5.31-5.46\left(\mathrm{~m}, 6 \mathrm{H}, 6{ }^{\prime}-\mathrm{H}, 10^{\prime}-\mathrm{H}\right.$,

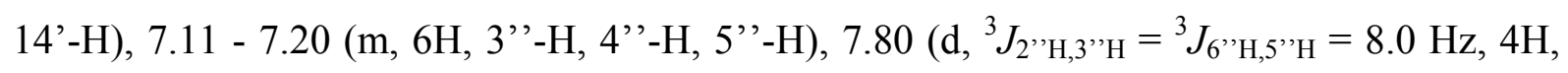
2 ',-H, 6',-H).

${ }^{19}$ F NMR $\left(470 \mathrm{MHz}, \mathrm{C}_{6} \mathrm{D}_{6}\right): \delta=-71.68\left(\mathrm{CF}_{3}\right)$.

$\mathrm{C}_{35} \mathrm{H}_{51} \mathrm{~F}_{3} \mathrm{O}_{3}$ (576.78) 


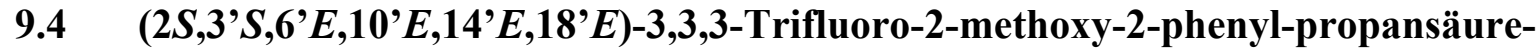
3',7',11',15',19'-pentamethyl-eicosa-6',10',14',18'-tetraenyl-ester [(S)-79]

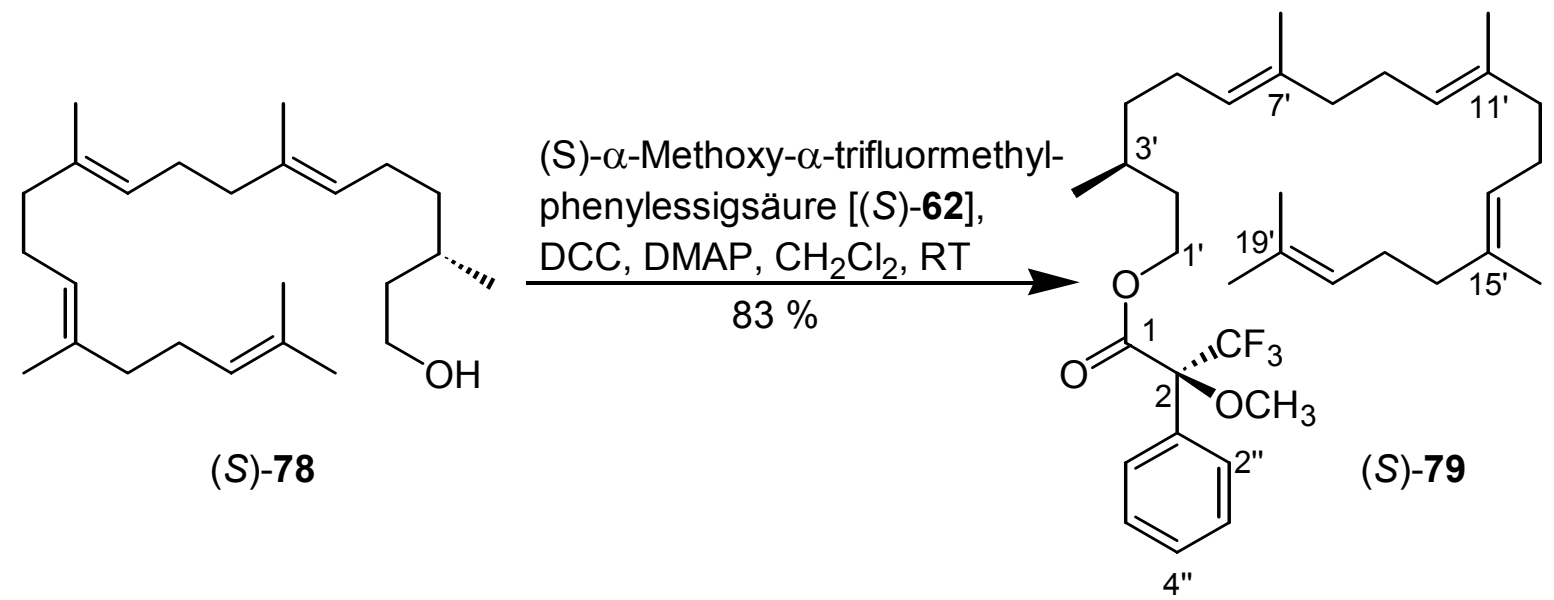

Es wurden $7.1 \mathrm{mg}$ (0.031 mmol. 1.1 Äquiv.) der enantiomerenreinen $(S)-(-) \alpha-M e t h o x y-\alpha-$ trifluormethylphenylessigsäure $\quad[(S)-62], \quad 6.3 \quad \mathrm{mg} \quad(0.031 \quad \mathrm{mmol}, \quad 1.1 \quad$ Äquiv. $)$ Dicyclohexylcarbodiimid und $0.5 \mathrm{mg}$ (0.004 mmol, 0.12 Äquiv.) 4-Dimethylaminopyridin mit $10.0 \mathrm{mg}$ (0.028 mmol, 1.0 Äquiv.) des enantiomerenreinen Alkohols $(S)$-78 zur Reaktion gebracht. Zur Aufarbeitung fügte man $200 \mathrm{mg}$ Kieselgel zur Reaktionsmischung, entfernte alles Flüchtige i. Vak. am Rotationsverdampfer und reinigte den Rückstand an $2 \mathrm{~g}$ Kieselgel (Eluent: $\mathrm{Et}_{2} \mathrm{O} / \mathrm{PE}=1$ : 100). Es wurden $12.9 \mathrm{mg}$ (83\% Ausbeute) der diastereomerenreinen Titelverbindung $(S, S)-79$ isoliert.

$\mathbf{R}_{\mathbf{f}}=0.76\left(\mathrm{Et}_{2} \mathrm{O} / \mathrm{PE}=1: 3\right)$.

${ }^{13}$ C NMR (125 MHz, $\mathrm{C}_{6} \mathrm{D}_{6}$, Diastereomer A): $\delta={ }^{+}+16.04$, ' $^{\prime}$ ' 16.10, '+' $16.12\left(7^{\prime}-\mathrm{CH}_{3}\right.$, $\left.11^{\prime}-\mathrm{CH}_{3}, 15^{\prime}-\mathrm{CH}_{3}\right)$, '+' $17.73\left(18^{\prime}-\mathrm{CH}_{3}\right)$, '+' $19.12\left(3^{\prime}-\mathrm{CH}_{3}\right)$, '-' $25.61(\mathrm{C}-5$ '), '+' $25.84(\mathrm{C}-$

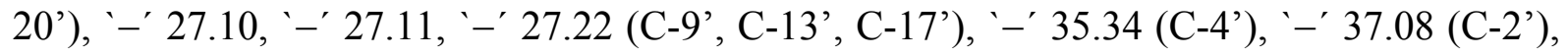
'-' $40.20\left(\mathrm{C}-8\right.$ ', C-12', C-16'), '+' $55.29\left(\mathrm{OCH}_{3}\right)$, '-' $64.71(\mathrm{C}-1$ ' $),{ }^{\prime}-{ }^{\prime} 88.11\left(\mathrm{~d},{ }^{2} J_{\mathrm{C}_{1, \mathrm{~F}}}=28\right.$ $\mathrm{Hz}, \mathrm{C}-2),{ }^{-}-124.26\left(\mathrm{q},{ }^{1} J_{\mathrm{CF}_{3}}=288 \mathrm{~Hz}, \mathrm{CF}_{3}\right)$, '+' 124.74 , '+' 124.77 , '+' 124.81 , '+' 124.92 (C-6', C-10', C-14', C-18'), '+' 127.76 (C-3'’ und C-5'’), '+' 128.58 (C-2', und C-6' '), '+' 129.67 (C-4"'), '-' 131.11 (C-19'), '-' 133.16 (C-1'”), '-' 135.01, '-' 135. 06, '-' 135.09 (C7', C-11', C-15'), '-' $166.60(\mathrm{C}=\mathrm{O})$.

$\mathrm{C}_{35} \mathrm{H}_{51} \mathrm{~F}_{3} \mathrm{O}_{3}$ (576.78) 


\section{Charakterisierung von Methanophenazin (1)}

Stamm: $\quad$ Ms. mazei Gö1; DSM-Stamm-Nr.: 3647; Methanophenazin (1)

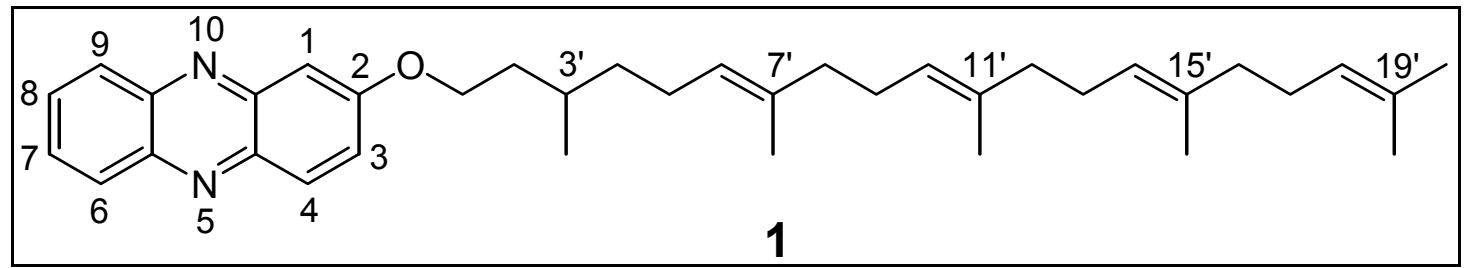

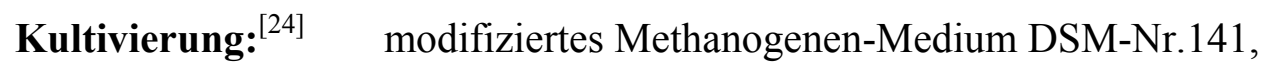
$150 \mathrm{mM}$ Methanol, $\mathrm{pH}$ 6.9-7.3, strikt anaerobe Bedingungen in $\mathrm{N}_{2} / \mathrm{CO}_{2}$-Atmosphäre, $37^{\circ} \mathrm{C}$ (siehe Kapitel 4 und Abbildung 20).

Aufarbeitung: ${ }^{[24]}$ Membranpräparation: Abtrennen der Cytoplasmamembranen durch Zentrifugation, Isooctanextraktion der Cytoplasmamembranen.

Reinigung: HPLC: semipräparative Kieselgelsäule (Si 60, 10 x 250 mm, Kontron); Flußrate: $4.0 \mathrm{ml} / \mathrm{min}$; Detektion: $\lambda=260 \mathrm{~nm}$; Eluent A: Cyclohexan; Eluent B: Ethylacetat; Eluentengradient: 0 bis $15 \min 5 \%$ B, 15 bis $22 \min 30 \%$ B, 22 bis 28 min stufenloser Gradient bis $100 \%$ B, 28 bis $35 \mathrm{~min} 5 \%$ B. Dabei wurden pro Lauf etwa $10 \mathrm{mg}$ Substanzgemisch in $200 \mu \mathrm{l}$ Isooctan auf die Säule aufgetragen.

Ausbeute: $\quad$ aus $5 \times 100 \mathrm{~g}$ Zellen wurden $1.2 \mathrm{mg} 1$ als gelbes Öl isoliert.

$\mathbf{R}_{\mathbf{f}}=0.51$ (Diethylether/Hexan $\left.=1: 1\right)$.

Detektion: UV-löschend bei $254 \mathrm{~nm}$, entfärbt $\mathrm{KMnO}_{4}$-Lösung.

${ }^{1} \mathbf{H}$ NMR (500 MHz, CD $\left.{ }_{3} \mathrm{OD}\right): \delta=1.04\left(\mathrm{~d},{ }^{3} \mathrm{~J}_{3}{ }^{\prime} \mathrm{CH}_{3}, 3^{\prime} \mathrm{H}=6.6 \mathrm{~Hz}, 3 \mathrm{H}, 3{ }^{\prime}-\mathrm{CH}_{3}\right), 1.27-1.37(\mathrm{~m}$, $\left.2 \mathrm{H}, 4^{\prime}-\mathrm{H}_{2}\right), 1.46-1.53(\mathrm{~m}, 1 \mathrm{H}, 3$ '- $-\mathrm{H}), 1.54,1.55,1.56$ (3s, je $3 \mathrm{H}, 11^{\prime}-\mathrm{CH}_{3}, 15^{\prime}-\mathrm{CH}_{3}, 19$ '$\left.\mathrm{CH}_{3}\right), 1.62\left(\mathrm{~s}, 3 \mathrm{H}, 7^{\prime}-\mathrm{CH}_{3}\right), 1.63\left(\mathrm{~d},{ }^{4} J_{20}{ }^{\prime} \mathrm{H}_{3}, 18^{\prime} \mathrm{H}=1.0 \mathrm{~Hz}, 3 \mathrm{H}, 20^{\prime}-\mathrm{H}_{3}\right), 1.70-1.79\left(\mathrm{~m}, 1 \mathrm{H}, 2^{\prime}-\right.$ $\left.\mathrm{H}_{\mathrm{B}}\right) 1.79-1.86\left(\mathrm{~m}, 1 \mathrm{H}, 2^{\prime}-\mathrm{H}_{\mathrm{A}}\right), 1.87-2.17\left(\mathrm{~m}, 14 \mathrm{H}, 5^{\prime}-\mathrm{H}_{2}, 8^{\prime} \mathrm{H}_{2}, 9^{\prime} \mathrm{H}_{2}, 12^{\prime}-\mathrm{H}_{2}, 13^{\prime}-\mathrm{H}_{2}, 16^{\prime}-\right.$ $\left.\mathrm{H}_{2}, 17^{\prime}-\mathrm{H}_{2}\right), 4.29\left(\mathrm{~m}_{\mathrm{C}}, 2 \mathrm{H}, 1^{\prime}-\mathrm{H}_{2}\right), 4.99-5.06\left(\mathrm{~m}, 2 \mathrm{H}, 10^{\prime}-\mathrm{H}, 14^{\prime}-\mathrm{H}\right), 5.08\left(\mathrm{t} \mathrm{br},{ }^{3} J_{6}{ }^{\prime} \mathrm{H}, 5^{\prime} \mathrm{H}_{2}=\right.$ $7.9 \mathrm{~Hz}$, aufgespalten durch Fernkopplungen $\left.{ }^{4} \mathrm{~J} \approx 1.1 \mathrm{~Hz}, 1 \mathrm{H}, 66^{\prime}-\mathrm{H}\right), 5.15\left(\mathrm{t} \mathrm{br},{ }^{3} J_{18^{\prime} \mathrm{H}, 17^{\prime} \mathrm{H}_{2}}=7.0\right.$ $\mathrm{Hz}$, aufgespalten durch Fernkopplungen $\left.{ }^{4} J \approx 1.2 \mathrm{~Hz}, 1 \mathrm{H}, 18^{\prime}-\mathrm{H}\right), 7.42\left(\mathrm{~d},{ }^{4} J_{1 \mathrm{H}, 3 \mathrm{H}}=2.4 \mathrm{~Hz}\right.$, $1 \mathrm{H}, 1-\mathrm{H}), 7.58\left(\mathrm{dd},{ }^{3} J_{3 \mathrm{H}, 4 \mathrm{H}}=9.4 \mathrm{~Hz},{ }^{4} J_{4 \mathrm{H}, 1 \mathrm{H}}=2.5 \mathrm{~Hz}, 1 \mathrm{H}, 3-\mathrm{H}\right) 7.85\left(\mathrm{ddd},{ }^{3} J_{7 \mathrm{H}, 6 \mathrm{H}}=8.4 \mathrm{~Hz}\right.$, 
${ }^{3} J_{7 \mathrm{H}, 8 \mathrm{H}}=6.7 \mathrm{~Hz},{ }^{4} J_{7 \mathrm{H}, 9 \mathrm{H}}=1.4 \mathrm{~Hz}, 1 \mathrm{H}, 7-\mathrm{H}$ oder $\left.8-\mathrm{H}\right), 7.91\left(\mathrm{ddd},{ }^{3} J_{8 \mathrm{H}, 9 \mathrm{H}}=8.5 \mathrm{~Hz},{ }^{4} J_{8 \mathrm{H}, 7 \mathrm{H}}=6.7\right.$ $\mathrm{Hz},{ }^{4} J_{8 \mathrm{H}, 6 \mathrm{H}}=1.5 \mathrm{~Hz}, 1 \mathrm{H}, 8-\mathrm{H}$ oder $\left.7-\mathrm{H}\right), 8.09\left(\mathrm{~d},{ }^{3} J_{4 \mathrm{H}, 3 \mathrm{H}}=9.4 \mathrm{~Hz}, 1 \mathrm{H}, 4-\mathrm{H}\right), 8.15\left(\mathrm{ddd},{ }^{3} J_{6 \mathrm{H}, 7 \mathrm{H}}\right.$ $=8.8 \mathrm{~Hz},{ }^{4} J_{6 \mathrm{H}, 8 \mathrm{H}}=0.8 \mathrm{~Hz},{ }^{5} J_{6 \mathrm{H}, 9 \mathrm{H}}=0.6 \mathrm{~Hz}, 1 \mathrm{H}, 6-\mathrm{H}$ oder $\left.9-\mathrm{H}\right), 8.19\left(\mathrm{dd},{ }^{3} J_{9 \mathrm{H}, 7 \mathrm{H}}=8.5 \mathrm{~Hz}\right.$, ${ }^{4} J_{9 \mathrm{H}, 7 \mathrm{H}}=1.4 \mathrm{~Hz},{ }^{5} J_{9 \mathrm{H}, 6 \mathrm{H}}=0.6 \mathrm{~Hz}, 1 \mathrm{H}, 9-\mathrm{H}$ oder $\left.6-\mathrm{H}\right)$.

${ }^{13} \mathrm{C}$ NMR (125 MHz, $\left.\mathrm{C}_{6} \mathrm{D}_{6}\right): \delta={ }^{+}+16.12$, ' +' 16.13, ' +' $16.15\left(7^{\prime}-\mathrm{CH}_{3}, 11\right.$ ' $\left.-\mathrm{CH}_{3}, 15^{\prime}-\mathrm{CH}_{3}\right)$,

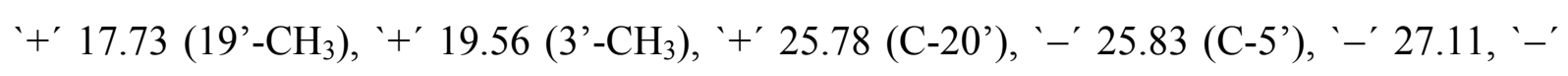
27.12 `-' 27.21 (C-9', C-13', C-17'), '+' 29.80 (C-3’), `-' 36.31 (C-2’), ’-' 37.45 (C-4'), '-' 40.20 und '-' 40.23 (C-8', C-12', C-16'), '-' 66.91 (C-1'), '+' 105.80 (C-1), '+' 124.73, '+' 124.77, '+' 124.93, (C-6', C-10', C-14'), ’+' 125.04 (C-18'), ’+' 126.53 (C-3), '+' 128.78 (C7 oder C-8), '+' 129.56 (C-8 oder C-7), '+' 130.17 (C-6 oder C-9), '+' 130.26 (C-9 oder C-6), '-' 131.07 (C-19'), '+' 131.29 (C-4), '-' 134.99, '-' 135.04, '-' 135.09 (C-7', C-11', C-15'), '-' 141.36 (C-10a), '-' 142.63 (C-5a oder C-9a), '-' 144.12 (C-9a oder C-5a), '-' 145.87 (C$4 \mathrm{a}),{ }^{\prime} ' 160.78(\mathrm{C}-2)$.

MS $(70 \mathrm{eV}): m / z(\%)=538(100)\left[\mathrm{M}^{+}\right], 470(6)\left[\mathrm{M}^{+}-\mathrm{C}_{5} \mathrm{H}_{8}\right], 402(8)\left[470-\mathrm{C}_{5} \mathrm{H}_{8}\right], 334(20)$ [402- $\left.\mathrm{C}_{5} \mathrm{H}_{8}\right], 265$ (4) [334- $\left.\mathrm{C}_{5} \mathrm{H}_{9}\right], 196(82)\left[\mathrm{C}_{12} \mathrm{H}_{8} \mathrm{~N}_{2} \mathrm{O}^{+}\right], 168$ (7) [196-CO].

HR-MS: ber. für $\mathrm{C}_{37} \mathrm{H}_{50} \mathrm{~N}_{2} \mathrm{O}: 538.3923$; gef.: 538.3923 .

\section{$\mathrm{C}_{37} \mathrm{H}_{50} \mathrm{~N}_{2} \mathrm{O}$ (538.39)}




\section{Anhang}




\section{Abkürzungsverzeichnis}

\begin{tabular}{|c|c|}
\hline APT & attached proton test \\
\hline Äquiv. & Moläquivalente \\
\hline ber. & berechnet \\
\hline $\mathrm{DC}$ & Dünnschichtchromatographie \\
\hline DCI & Direct Chemical Ionisation \\
\hline DME & Dimethoxyethan \\
\hline $\mathrm{DMF}$ & Dimethylformamid \\
\hline DMSO & Dimethylsulfoxid \\
\hline DIBAH & Diisobutylaluminiumhydrid \\
\hline EI & Elektronenstoßionisation \\
\hline Et & Ethyl \\
\hline gef. & gefunden \\
\hline $\mathrm{h}$ & Stunde(n) \\
\hline HPLC & High Performance Liquid Chromatography \\
\hline LiTMP & Lithium-2,2,6,6-tetramethylpiperidid \\
\hline $\mathrm{Me}$ & Methyl \\
\hline $\min$ & Minuten \\
\hline MS & Massenspektrum \\
\hline NMR & Nuclear Magnetic Resonanz \\
\hline$o-, m-, p-$ & ortho-, meta-, para- \\
\hline $\mathrm{PE}$ & Petrolether \\
\hline $\mathrm{Ph}$ & Phenyl \\
\hline Schmp & Schmelzpunkt \\
\hline TBAF & Tetrabutylammoniumfluorid \\
\hline TBDMS & tert.-Butyldimethylsilyl \\
\hline Temp. & Temperatur \\
\hline THF & Tetrahydrofuran \\
\hline tert.- & tertiär \\
\hline
\end{tabular}




\section{Literaturverzeichnis}

[1] H. G. Schlegel, Allgemeine Mikrobiologie, 7. überarb. Aufl., Thieme, Stuttgart, 1992.

[2] D. Voet, J. G. Voet, Biochemie (Hrsg.: A. Maelike, W. Müller-Esterl), VCH, Weinheim, 1992.

[3] G. Gottschalk, Bacterial metabolism, $2^{\text {nd }}$ ed., Springer, New York, 1986.

[4] J. Koolmann, K.-H. Röhm, Taschenatlas der Biochemie, Thieme, Stuttgart, 1994.

[5] T. D. Brock, M. T. Madigan, J. M. Martinko, J. Parker, Biology of Microorganisms, Prentice-Hall International, London, 1994.

[6] H. Cypionka, Grundlagen der Mikrobiologie, Springer, Berlin, 1999.

[7] S. H. Zinder in Methanogenesis (Hrsg.: J. G. Ferry), Chapman \& Hall, New York, 1993, S. 128.

[8] R. Cammak, Nature 1997, 443.

[9] O. Kandler, H. König, Arch. Microbiol. 1978, 118, 141.

[10] T. G. Tornabene, T. A. Langworthy, Science 1987, 203, 51.

[11] W. Zillig, K. O. Stetter, R. Schnabel, J. Madou, A. Gierl, Zbl. Bakt. Hyg. 1 Abt. Orig. C3 1982, 218.

[12] H. Cypionka, Grundlagen der Mikrobiologie, Springer, Berlin, 1999, S. 76.

[13] D. R. Boone, W. B. Whitman, P. E. Rouviere in Methanogenesis (Hrsg.: J. G. Ferry), Chapman \& Hall, New York, 1993, S. 35.

[14] U. Deppenmeier, A. Blaut, G. Gottschalk, Eur. J. Biochem. 1989, 186, 317.

[15] J. Escalanta-Semerenda, K. L. Rinehart, R. S. Wolfe, J. Biol. Chem. 1984, 259, 9447.

[16] R. K. Thauer, Microbiology 1998, 144, 2377.

[17] U. Deppenmeier, T. Lienhard, G. Gottschalk, FEBS Lett. 1999, 457, 291.

[18] U. Deppenmeier, M. Blaut, G. Gottschalk, Arch. Microbiol. 1991, 155, 272.

[19] U. Harms, R. K. Thauer, Eur. J. Biochem. 1996, 235, 653.

[20] A. Mahlmann, U. Deppenmeier, G. Gottschalk, FEMS Microbiol. Lett. 1989, 61, 149; U. Harms, R. K. Thauer, Eur. J. Biochem. 1996, 241, 149. 
[21] K. Ma, R. K. Thauer, FEMS Microbiol. Lett. 1990, 70, 119; B. W. J. Te Broemmelstroet, W. J. Geerts, J. T. Keltjens, C. van der Drift, G. D. Vogels, Biochim. Biophys. Acta 1991, 1079, 293.

[22] Für die Abbildung danke ich Herrn Priv.-Doz. Dr. U. Deppenmeier.

[23] U. Deppenmeier, T. Lienhard, G. Gottschalk, FEBS Lett. 1999, 457, 291.

[24] H. J. Abken, Dissertation, Universität Göttingen, 1997.

[25] H. J. Abken, U. Deppenmeier, FEMS Microbiol. Lett. 1997, 154, 231.

[26] S. Bäumer, T. Ide, C. Jacobi, A. Johann, G. Gottschalk, U. Deppenmeier, J. Biol. Chem. 2000, 275, 17968.

[27] U. Deppenmeier, M. Blaut, B. Schmitt, G. Gottschalk, Arch. Microbiol. 1992, $157,505$.

[28] H. J. Abken, M. Tietze, J. Brodersen, S. Bäumer, U. Beifuss, U. Deppenmeier, J. Bacteriol. 1998, 180, 2027.

[29] U. Beifuss, M. Tietze, S. Bäumer, U. Deppenmeier, Angew. Chem. 2000, 112, 2583; Angew. Chem. Int. Ed. 2000, 39, 2470.

[30] E. Negishi, D. E. Van Horn, A. O. King, N. Okukado, Synthesis 1979, 501; D. E. Van Horn, E. Negishi, J. Am. Chem. Soc. 1978, 100, 2252.

[31] F. Kehrmann, F. Cherpillod, Helv. Chim. Acta 1924, 7, 973.

[32] persönliche Mitteilung Dr. G. Remberg, Universität Göttingen, 1998.

[33] The Aldrich Library of ${ }^{13} C$ and ${ }^{1} H$ FT NMR-Spectra 1993, 2, 1276.

[34] W. Gottwald, RP-HPLC für Anwender, VCH, Weinheim, 1993.

[35] R. H. Thomson in Naturally Occuring Quinones III, Chapman \& Hall, London, 1987, S. 55.

[36] F. W. McLafferty, F. Turecek, Interpretation von Massenspektren, Spektrum, Heidelberg, 1995, S. 27.

[37] H. O. Kalinowski, S. Berger, S. Braun, ${ }^{13} C$-NMR-Spektroskopie, Thieme, Stuttgart, 1984, S. 117.

[38] L. Jaenicke, H.-U. Siegmund, Chemistry and Physics of Lipids 1989, 51, 159; Y. Tanaka, H. Sato, A. Kageyu, T. Tomita, Biochem. J 1987, 243, 481; Y. Tanaka, H. Sato, A. Kageyu, Polymer 1982, 23, 1087; D. Grassi, V. Lippuner, J. Brunner, A. Vasella, J. Am. Chem. Soc. 1997, 119, 10992.

[39] eine gute Übersicht zu diesem Thema geben J. M. Turner, A. J. Messenger, Adv. Microb. Physiol 1986, 27, 211. 
[40] J. Fordos, Recueil des Travaux de la Societe d'Emulation pour les Siences Pharmaceutiques 1859, 3, 30; F. Wrede, E. Starck, Z. Physiol. Chem. 1929, $181,58$.

[41] L. Guignard, M. Sauvageau, Compte Rendu des Siences de la Societe de Biologie 1894, 46, 841.

[42] J. R. Clemo, H. McIlwain, J. Chem. Soc. 1938, 479; J. R. Clemo, H. McIlwain, J. Chem. Soc. 1950, 1481.

[43] M. McDonald, B. Wilkinson, C. W. Van't Land, U. Mocek, S. Lee, H. G. Floss, J. Am. Chem. Soc. 1999, 121, 5619.

[44] A. Römer, H. Budzikiewicz, H. Korth, G. Pulverer, Tetrahedron Lett. 1979, 509;

A. Römer, H. Scholl, H. Budzikiewicz, Z. Naturforsch. B: Chem. Sci 1981, 36, 1037;

M. E. Levich, P. Reitz, Biochemistry 1966, 5, 689.

[45] U. Hollstein, R. J. Van Gemert, Biochemistry 1971, 10, 497; U. Hollstein, P. I. Butler, Biochemistry 1972, 11, 1345; H. M. Hassan, I. Friedovich, J. Bacteriol. 1980, 141, 156.

[46] S. Yamanaka, Chiba Igakkai Zasshi 1972, 48, 63 (Chem. Abstr. 1972, 77, 162986).

[47] U. Gräfe, Biochemie der Antibiotika, Spektrum, Heidelberg, 1992, S. 65.

[48] Kyowa Hakko Kogyo Co. Ltd. 1982, japanisches Patent 82-04, 975 (Chem. Abstr. 1982, 96, 197887f).

[49] W. Keller-Schierlein, A. Geiger, H. Zähner, M. Brandl, Helv. Chim. Acta 1988, 71, 2058; A. Geiger, W. Keller-Schierlein, M. Brandl, H. Zähner, J. Antibiot. 1988, 41,1542 .

[50] R. B. Herbert, F. G. Holliman, P. N. Ibberson, J. B. Sheridan, J. Chem. Soc., Perkin Trans. 1 1979, 2411; T. E: Etherington, R. B. Herbert, F. G. Holliman, J. B. Sheridan, J. Chem. Soc., Perkin Trans. 1 1979, 2416; U. Hollstein, L. G. Marshall, J. Org. Chem. 1972, 37, 3510.

[51] A. M. Helbing, M. Viscontini, Helv. Chim. Acta 1976, 59, 2284; A. G. McInnes, D. G. Smith, J. A. Walter, Can. J. Chem. 1979, 57, 3200; J. L. C. Wright, L. C. Vining, A. G. McInnes, Can. J. Biochem. 1977, 55, 678; E. Leete, N. Kowanko, R. A. Newmark, Tetrahedron Lett. 1975, 4103; M. Tanabe, S. Urano, Tetrahedron 1983, $39,3569$. 
[52] M. Podojil, N. N. Gerber, Biochemistry 1967, 6, 2701; M. Podojil, N. N. Gerber, Biochemistry 1970, 9, 4616; R. B. Herbert, F. G. Holliman, P. N. Ibberson, Tetrahedron Lett. 1974, 151; R. B. Herbert, F. G. Holliman, G. B. Sheridan, Tetrahedron Lett. 1974, 4201; R. B. Herbert, F. G. Holliman, G. B. Ibberson, Tetrahedron Lett. 1976, 639; U. Hollstein, D. I. Mock, R. R. Sibbit, U. Roitch, F. Lingens, Tetrahedron Lett. 1978, 2987.

[53] D. H. Calhoun, M. Carson, R. A. Jensen, J. Gen. Microbiol. 1972, 72, 581; R. P. Longley, J. E. Haliwell, J. J. R. Campell, W. M. Ingelsdew, Can. J. Microbiol. $1972,18,1357$.

[54] A. Römer, R. B. Herbert, Z. Naturforsch., C: Biosci. 1982, 37, 1070;

R. B. Herbert, J. Mann, A. Römer, Z. Naturforsch., C: Biosci. 1982, 37, 159.

[55] A. J. M. Messenger, J. M. Turner, FEMS Microbiol. Lett. 1983, 18, 65; A. Römer, E. Lange, Z. Naturforsch., C: Biosci. 1983, 38, 539; U. Hollstein, G. E. Krisov, D. L. Mock, Tetrahedron Lett. 1976, 3267; P. R. Buckland, R. B. Herbert, F. G. Holliman; J. of Chem. Res., Synop. 1981, 362.

[56] A. J. M. Messenger, J. M. Turner, Biochem. Soc. Trans. 1978, 6, 1326;

S. P. Gulliford, R. B. Herbert, F. G. Holliman, Tetrahedron Lett. 1978, 2, 195.

[57] E. D. Weinberg, Adv. Microbiol Physiol. 1970, 4, 1; E. D. Weinberg, Perspectives in Biology and Medicine 1971, 14, 565.

[58] J. F. Martin, A. I. Demain, Microbiol. Rev. 1980, 44, 230.

[59] J. M. Turner, A. J. Messenger, Adv. Microbiol. Physiol. 1986, 27, 218.

[60] U. Orleb, Methoden Org. Chemie (Houben-Weyl), 4. Aufl., Bd. E9b/2, 1998, S. 266; N. Hughes in Rodd's Chemistry of Carbon Compounds (Hrsg.: S. Coffey, M. F. Ansell), $2^{\text {nd }}$ ed., Vol. IV, Part I J, Elsevier, Amsterdam, 1989, S 403.

[61] F. Kehrmann, F. Cherpillod, Helv. Chem. Acta 1924, 7, 973; F. Kehrmann, C. Mermod, Helv. Chim. Acta 1927, 10, 62; C. Liebermann, O. N. Witt, Ber. Dtsch. Chem. Ges. 1887, 20, 2442; T. G. H. Jones, R. Robinson, J. Chem. Soc. 1917, 111, 927.

[62] C. Ris, Ber. Dtsch. Chem. Ges. 1886, 19, 2206.

[63] D. L. Vivian, H. C. Waterman, J. Org. Chem. 1949, 14, 289.

[64] R. Nietzky, O. Ernst, Ber. Dtsch. Chem. Ges. 1890, 23, 1852. 
[65] D. L. Vivian, J. L. Hartwell, H. C. Waterman, J. Org. Chem. 1953, 19, 1136;

D. L. Vivian, J. Am. Chem. Soc. 1951, 73, 457.

[66] Übersicht über die Reaktionen von Benzofuroxan: A. Gasco, A. J. Boulton, Adv. Heterocycl. Chem. 1981, 29, 251 und K. Ley, F. Seng, Synthesis 1975, 415.

[67] K. Ley, F. Seng, U. Eholzer, R. Nast, R. Schubert, Angew. Chem. 1969, 81, 569; Angew. Chem. Int. Ed. 1969, 8, 596.

[68] F. Kehrmann, F. Cherpillod, Helv. Chim. Acta 1924, 7, 973.

[69] R. Ott, Monatsh. Chem. 1959, 90, 827.

[70] J. Thiele, Ber. Dtsch. Chem. Ges. 1898, 1, 1247.

[71] R. Willstätter, F. Müller, Ber. Dtsch. Chem. Ges 1911, 44, 2180.

[72] Y. Morita, Chem. Pharm. Bull. 1966, 14(4), 426.

[73] P. J. Kocienski, G. Cernigliaro, G. Feldstein, J. Org. Chem. 1977, 42, 353.

[74] A. Loupy, J. Sansoulet, F. Vaziri-Zand, Bull. Soc. Chim. Fr. 1987, 1027.

[75] P. G. McDougal, J. G. Rico, Y.-I. Oh, B. D. Condon, J. Org. Chem. 1986, 51, 3388.

[76] U. Jensen-Korte, H.-J. Schäfer, Liebigs Ann. Chem. 1982, 1532; R. Rossi, A. Carpita, M. Chini, Tetrahedron 1985, 41, 627; K. Mori, S. Masuda, T. Suguro, Tetrahedron 1981, 37, 1329.

[77] D. B. Collum, J. H. McDonald III, W.C. Still, J. Am. Chem. Soc. 1980, 102, 2120.

[78] H. G. W. Leuenberger, W. Boguth, R. Barner, M. Schmid R. Zell, Helv. Chim. Acta 1979, 62, 455.

[79] P. Mohr, M. Tori, P. Grossen, P. Herold, C. Tamm, Helv. Chim. Acta 1982, 65, 1412;

P. Herold, P. Mohr, C. Tamm, Helv. Chim. Acta 1983, 66, 744.

[80] S. Ställberg-Stenhagen, Arkiv Kemi Mineral. Geol. 1947, 25A, No. 10, 1.

[81] R. Chenevert, M. Desjardins, Tetrahedron Lett. 1991, 32, 4249.

[82] L. K. P. Lam, R. A. H. F. Hui, J. B. Jones, J. Org. Chem. 1986, 51, 2047.

[83] A. J. Irvin, J. B. Jones, J. Am. Chem. Soc. 1977, 99, 556.

[84] P. Herold, P. Mohr, C. Tamm, Helv. Chim. Acta 1983, 66, 744.

[85] N. M. Yoon, C. S. Pak, H. C. Brown, S. Krishnamurthy, T. P. Stocky, J. Org. Chem. 1973, 38, 2786.

[86] C. Tamm weist in seiner Publikation [84] auf dieses Problem hin.

[87] C. F. Lane, Aldrichimica Acta, 1975, 8, 20. 
[88] R. Rossi, A. Carpita, M. Chini, Tetrahedron 1985, 41, 627; C. P. D. Theisen, C. H. Heathcock, J. Org. Chem. 1993, 58, 142.

[89] R. Brückner, Reaktionsmechanismen, 1. Aufl., Spektrum, Heidelberg, 1996, S. 525.

[90] L.-Y. Chen, A. Zaks, S. Chackalamannil, S. Dugar, J. Org. Chem. 1996, 61, 8341.

[91] D. Terunuma, M. Motegi, M. Tsuada, T. Sawada, H. Nozawa, H. Nohira, J. Org. Chem. 1987, 52, 1630.

[92] H. C. Brown, S. C. Kim, Synthesis 1977, 635.

[93] G. B. Fisher, J. C. Fuller, J. Harrison, S. G. Alvarez, E. R. Burkhardt, C. T. Golarski, B. Singaram, J. Org. Chem. 1994, 59, 6378.

[94] A. G. Myers, B. H. Yang, H. Chen, L. McKinstry, D. J. Kopecky, J. L. Gleason, J. Am.

Chem. Soc. 1997, 119, 6496.

[95] J. A. Dale, D. L. Dull, H. S. Mosher, J. Org. Chem. 1969, 34, 2543; J. A. Dale, H. S. Mosher, J. Am. Chem. Soc. 1973, 95, 512.

[96] D. E. Van Horn, E. Negishi, J. Am. Chem. Soc. 1978, 100, 2252.

[97] Dr. J. Paust und Dr. H. Jaedicke (BASF AG, Ludwigshafen) sowie Dr. R. K. Müller (Hoffmann-La Roche Ltd., Basel) danke ich für die großzügigen Spenden von $(E, E)$ Farnesylaceton.

[98] ausführliche Übersichten zur Organozirkonium-Chemie: E. Negishi, T. Takahashi, Synthesis 1988, 1; E. Negishi, T. Takahashi, Aldrichim. Acta 1985, 18, 31; E. Negishi, Pure Appl. Chem. 1981, 53, 2333.

[99] E. Negishi, A. O. King, W. L. Klima, J. Org. Chem. 1980, 45, 2526.

[100] Org. Synth. 1985, 64, 44.

[101] E. Negishi, D. E. Van Horn, A. O. King, N. Okukado, Synthesis 1979, 501.

[102] T. Yoshida, E. Negishi, J. Am. Chem. Soc. 1981, 103, 1276; T. Yoshida, E. Negishi, J. Am. Chem. Soc. 1981, 103, 4958.

[103] S. V. Ley, A. Armstrong, D. Diez-Martin, M. J. Ford, P. Grice, J. G. Knight, H. C. Kolb, A. Madin, C. A. Marby, S. Mukherjee, A. N. Shaw, A. M. Z. Slawin, S. Vile, A. D. White, D. J. Williams, M. Woods, J. Chem. Soc., Perkin Trans. 1 1991, 667.

[104] K. Mori, N. Murata, Liebigs Ann. 1995, 12, 2089.

[105] A. B. Smith III, Y. Qiu, D. R. Jones, K. Kobayashi, J. Am. Chem. Soc. 1995, 117, 12011. 
[106] L. S. Hegedus, Organische Synthese mit Übergangsmetallen, VCH, Weinheim, 1995, S. 73.

[107] Wenn sich nur ein Isomer nachweisen läßt, wird eine Reinheit von $>95 \%$ angegeben.

[108] G. Snatzke, ChiuZ 1981, 15, 78.

[109] Für die Durchführung dieser Arbeiten danke ich Herrn Michael Dehler; M. Dehler, Staatsexamensarbeit, Universität Bayreuth, 1998.

[110] S. Bäumer, E. Murakami, J. Brodersen, G. Gottschalk, S. W. Ragsdale, U. Deppenmeier, FEBS Lett. 1998, 428, 295.

[111] D. D. Perrin, W. L. F. Armarego, Purification of Laboratory Chemicals, $3^{\text {rd }}$ ed., Pergamon, Oxford, 1988.

[112] W. C. Still, M. Kahn, J. Org. Chem. 1978, 43, 2923.

[113] Die genaue Konzentration der verwendeten $n$-Buthylithium Lösung wurde durch direkte Titration einer Lösung von $N$-Pivaloyl-o-toluidin in THF bestimmt, siehe dazu J. Suffert, J. Org. Chem. 1989, 54, 509.

[114] Übersicht: E. Negishi, F. Liu in Metal-catalysed Cross-coupling Reactions (Hrsg.:

F. Diederich, P. J. Stang), Wiley-VCH, Weinheim, 1998, S. 1.

[115] D. Terunuma, M. Motegi, M. Tsuda, T. Sawada, H. Nozawa, H. Nohira, J. Org. Chem. 1987, 52, 1630 .

[116] R. W. Hoffmann, B. Landmann, Chem. Ber. 1986, 119, 1039.

[117] T. H. Chan, K. Koumaglo, J. Organomet. Chem. 1985, 285, 109.

[118] Chromatographic Chiral Separations (Hrsg.: M. Zief, L. J. Crane), Marcel Dekker, New York, 1988.

[119] Chiral Liquid Chromatography (Hrsg.: W. J. Lough), Chapman \& Hall, New York, 1996.

[120] A. A. DiMarco, T. A. Bobik, R. S. Wolfe, Annu. Rev. Biochem. 1990, 59, 355;

L. D. Eirich, G. D. Vogels, R. S. Wolfe, Biochemistry 1978, 17, 4583; L. G. M. Gorris, C. van der Drift, BioFactors 1994, 4, 139; P. J. Keller, H. G. Floss, J. Am. Chem. Soc. 1986, 108, 344; D. W. Sullins, T. A. Bobik, R. S. Wolfe, K. L. Rinehart, J. Am. Chem. Soc. 1993, 115, 6646.

[121] D. E. Cane, G. Young, J. Org. Chem. 1994, 59, 5794.

[122] D. Raederstorff, A. Y. L. Shu, E. J. Thompson, G. Djerassi, J. Org. Chem. 1987, 52, 2337. 
Meine akademischen Lehrer waren u. a. die Herren Professoren und Dozenten U. Beifuss, R. Brückner, U. Deppenmeier, G. Gottschalk, K. Hafner, H. Hippler, K. H. Hoyermann, G. F. Kahl, W. Knepel, H. Laatsch, F. W. Lichtenthaler, A. de Meijere, H. W. Roesky, C. Schneider, L. F. Tietze, H. G. Wagner, K. G. Weil, A. Zeeck. 


\section{Danksagung}

Der NMR-Abteilung des Hauses danke ich für die Aufnahme zahlreicher Protonen- und Kohlenstoff-Spektren. Mein besonderer Dank gilt Frau C. Zolke für die Durchführung der Sondermessungen, ihre liebenswürdige Art, eilige Aufträge auf dem „kleinen Dienstweg“ zu erledigen und ihr großes Herz für kleine Substanzmengen. Herrn Dipl.-Chem. R. Machinek danke ich für seine Bereitschaft zur ausführlichen Diskussion von Kernresonanz-Problemen. Mein Dank gebührt Frau G. Udvarnoki und Herrn Dr. G. Remberg für die Aufnahme der Massenspektren. Herrn Dr. G. Remberg danke ich zudem für viele wertvolle Tips beim Einrichten und Betreiben eines Gaschromatographen, sowie für die „Dauerleihgabe“ einer Chrompak SE 30 Kapillarsäule. Frau E. Pfeil verdanke ich die Aufnahmen der UV- und IRSpektren und die Messung der Drehwerte.

Den Mitgliedern des Arbeitskreises Deppenmeier, den Damen Katja Weiß, Claudia Hemmerling, Vera Allerheiligen, Anja Claus, Tina Ide und Sabine Lentes, sowie den Herren Hans-Jörg Abken, Sebastian Bäumer, Jens Brodersen und Frank Falinski danke ich für die wohlwollende Aufnahme und die erfolgreiche interdisziplinäre Zusammenarbeit. Herrn Priv.Doz. Dr. Uwe Deppenmeier danke ich für das geduldige Beantworten vieler Fragen und für meine Aufnahme in den Stand eines ,assoziierten Arbeitskreis-Mitglieds“.

Meinen Laborkollegen Olaf Kunz, Gerald Feder, Frank Döring, Heiko Leutbecher und Dietmar Schmidt danke ich für die ausgezeichnete Zusammenarbeit, die angenehme Arbeitsatmosphäre und die zahlreichen konstruktiven Gespräche, die wesentlich zum Gelingen dieser Arbeit beigetragen haben.

Ein besonderer Dank gilt Herrn Priv.-Doz. Dr. Uwe Beifuss für seine Unterstützung und Förderung während meiner gesamten Promotionszeit.

Bei meinen Freunden Dirk und Markus bedanke ich mich für ihren selbstlosen Einsatz beim Beheben rechnergestützter Schwierigkeiten und für das allzeit „,freie Sofa“. 


\section{LEBENSLAUF}

Persönliche Daten: Mario Tietze

geboren am 7. Juni 1966 als Sohn des Heizungsbauers

Manfred Tietze und seiner Frau Helga Tietze geb. Arend

in Kyllburg

\section{Schulausbildung:}

1972 bis 1976

1976 bis 1985

22. Juni 1985

Besuch der St. Maximin-Grundschule in Kyllburg Besuch des St. Matthias-Gymnasiums in Gerolstein Abiturprüfung

\section{Wehrdienst:}

1985 bis 1986

Wehrdienst im FschJgBtl 261 in Lebach

\section{Berufsausbildung:}

1986 bis 1989

Ausbildung zum Chemielaboranten bei der Hoechst AG in Frankfurt/M

13. Juni 1989

Ablegen der Prüfung vor der Industrie- und Handelskammer in Frankfurt/M

\section{Hochschulausbildung:}

SS 1989 bis SS 1991

30. September 1991

Chemiestudium an der Technischen Hochschule in Darmstadt

WS 1991 bis WS 1995

Diplom-Chemiker-Vorprüfung

Aug. 94 bis Mrz 95

Fortsetzung des Chemiestudiums an der Georg-August-

Universität in Göttingen

Aug. 94 bis Mrz. 95 Diplomarbeit am Institut für Organische Chemie der Universität Göttingen unter der Leitung von Dr. U. Beifuss unter der Schirmherrschaft von Prof. Dr. Dr. h.c. L.- F. Tietze zum Thema „Ausgewählte Transformationen mit 4-Silyloxy1-benzothiopyrylium-Salzen“"

3. November 1995 Diplom-Chemiker-Hauptprüfung Jan. 96 bis Okt. 97

Okt. 97 bis Mai 00 Untersuchungen zur biologischen Oxidation durch membrangebundene Dehydrogenasen des Essigsäurebakteriums Gluconobacter oxydans

Dissertation im Arbeitskreis von Priv.-Doz. Dr. U. Beifuss zum Thema „Methanophenazin: Strukturaufklärung und Totalsynthese eines neuartigen Cofaktors aus methanogenen Archaea"

2. November 2000 mündliche Promotionsprüfung 
